\title{
Game Design by Numbers:
}

Instrumental Play and the Quantitative Shift in the Digital Game Industry

by

Jennifer R. Whitson

A thesis submitted to the Faculty of Graduate and Postdoctoral Affairs in partial fulfilment of the requirements for the degree of

\author{
Doctor of Philosophy \\ in \\ Sociology \\ Carleton University, \\ Ottawa, Ontario
}

(C) 2012

Jennifer R. Whitson 
Library and Archives

Canada

Published Heritage

Branch

395 Wellington Street

Ottawa ON K1A ON4

Canada
Bibliothèque et

Archives Canada

Direction du

Patrimoine de l'édition

395 , rue Wellington

Ottawa ON K1A ON4

Canada
Your file Votre référence

ISBN: 978-0-494-94236-9

Our file Notre référence

ISBN: $978-0-494-94236-9$
NOTICE:

The author has granted a nonexclusive license allowing Library and Archives Canada to reproduce, publish, archive, preserve, conserve, communicate to the public by telecommunication or on the Internet, loan, distrbute and sell theses worldwide, for commercial or noncommercial purposes, in microform, paper, electronic and/or any other formats.

The author retains copyright ownership and moral rights in this thesis. Neither the thesis nor substantial extracts from it may be printed or otherwise reproduced without the author's permission.
AVIS:

L'auteur a accordé une licence non exclusive permettant à la Bibliothèque et Archives Canada de reproduire, publier, archiver, sauvegarder, conserver, transmettre au public par télécommunication ou par l'Internet, prêter, distribuer et vendre des thèses partout dans le monde, à des fins commerciales ou autres, sur support microforme, papier, électronique et/ou autres formats.

L'auteur conserve la propriété du droit d'auteur et des droits moraux qui protege cette thèse. $\mathrm{Ni}$ la thèse ni des extraits substantiels de celle-ci ne doivent être imprimés ou autrement reproduits sans son autorisation.
In compliance with the Canadian Privacy Act some supporting forms may have been removed from this thesis.

While these forms may be included in the document page count, their removal does not represent any loss of content from the thesis.
Conformément à la loi canadienne sur la protection de la vie privée, quelques formulaires secondaires ont été enlevés de cette thèse.

Bien que ces formulaires aient inclus dans la pagination, il n'y aura aucun contenu manquant. 


\begin{abstract}
This dissertation chronicles ideological, technological and economic changes in the digital game industry, focusing on how games are transforming as play becomes instrumentalized. It pays particular attention to the struggles of developers as they search for creative freedom and autonomy in a risk-averse industry. It makes original contributions to the literature on games by situating and explaining industry-wide shifts in terms of the socio-economics of game development and the rationalities that drive individual developers. It contributes to social theory more generally by explaining how transformations in play, games, and creativity are linked to much wider adaptations in the operation of capitalism and how it is justified to both workers and consumers.

I use ground-level accounts from those within the game industry to describe how new media technologies interact with socio-economic forces, detailing the adaptability of capitalist modes of production in the face of critique. I show how definitions of 'games' and 'play' are changing as they come into contact with technology, allowing games to be reformulated in powerful new ways, so games are not only tools of entertainment but also tools of governance. I argue that the collective valuation of objective quantitative data and the belief in the fallibility of individual creative autonomy has turned game design into "design by numbers".

The complementary themes of this thesis are bound together by references to the "New Spirit of Capitalism" (Boltanski and Chiapello 2007, 2005), which explains how capitalism is continually reorganizing itself, adapting the language and spirit of 1960s counterculture and emphasizing freedom in order to drive though new, more efficient, work practices and more subtle forms of exploitation. This "New Spirit" accounts for the current upheavals in the game industry. Changes to the Spirit of Capitalism have initiated tectonic shifts, reforming the geography of the game industry and creating fissures in the landscape that allow new game sectors to emerge, while others struggle to avoid being buried. In turn, innovations from the game industry, particularly the emphasis on datadriven design, shore up the weaknesses in the New Spirit of Capitalism, allowing it to operate more successfully.
\end{abstract}

\title{
keywords:
}

digital games; game development; game industry; Instrumentalization of Play; New Spirit of Capitalism; governance; surveillance 


\section{Acknowledgements}

I would like to thank Aron Kyle for being a staunch and stalwart supporter of my cause. I'm not sure he ever clued in that he was the key impetus for my focus on software developers. Perhaps it's better this way. I may continue observing, incognito.

This journey was made immeasurably easier by my defence committee and examiners: Robert Biddle, Brian Greenspan, Sheryl Hamilton, Aphra Kerr, and Bart Simon. Many other people provided inspiration along the way, including Kevin Haggerty, Justin Piché, Jessica Aldred, Chris Eaket, Natalie King, Elise Vist, Lauren Burr, Phil Horwitz, Trish Corrigan, Pippin Barr, Rilla Khaled, Jason Della Rocca, and too many others to name. I want to also thank my department's administrators, Paula Whissell, Karen Tucker, and Marlene Brancato.

I am eternally thankful for financial support from a number of bodies, including: the Social Sciences and Humanities Research Council of Canada (SSHRC); Carleton University's Hypertext and Hypermedia Lab; the Graphics, Animation, and New Media Network of Centres of Excellence (GRAND NCE); and Carleton's Human Oriented Technology Laboratory (HOTLab). I never once lacked intriguing research projects to work on and fascinating people to work with. In particular, the Hyperlab and HotLab and their founders - gave this sociology orphan a home in the digital media world. I am grateful.

My habit of periodically changing both my research topic and theoretical framework might have been met with disastrous consequences. I would like to thank my supervisor, Aaron Doyle, for his iron constitution. He took this all in stride and always offered unconditional support. 


\begin{tabular}{|l|c|}
\hline \multicolumn{2}{|c|}{ Table of Contents } \\
\hline Preliminary Section & ii \\
\hline Abstract & ii \\
\hline Acknowledgements & ii \\
\hline Table of Contents & iv \\
\hline List of Tables & viii \\
\hline List of Illustrations & viii \\
\hline List of Appendices & viii \\
\hline 1. Challenges and Change in the Digital Game Industry & 1 \\
\hline 1.1 Industry Upheavals & 3 \\
\hline 1.2 Why Study Games? & 6 \\
\hline 1.3 A Brief Overview of Game Studies & 9 \\
\hline 1.4 Thesis Overview & 17 \\
\hline Part I. Theorizing and Researching Game Development & 17 \\
\hline Part II. The Game Development Landscape & 20 \\
\hline Part III. Welcome to the Projective Cité & 23 \\
\hline Part I. Theorizing and Researching Game Development & 27 \\
\hline 2. The New Spirit of Capitalism in the Game Industry & 28 \\
\hline 2.1 Shifts in the Spirit of Capitalism & 29 \\
\hline 2.2 Defining the Cité & 32 \\
\hline 2.3 The Role of Criticism and Adaptations to Capitalism & 34 \\
\hline 2.4 Critiquing the New Spirit of Capitalism & 35 \\
\hline 2.5 Applying the New Spirit of Capitalism to the Game Industry & 40 \\
\hline 3. Games, Gamification and Governance & 43 \\
\hline 3.1 The New Spirit of Capitalism and Governing Through Freedom & 43 \\
\hline 3.2 Gamification & 50 \\
\hline The Emergence of Gamification & 52 \\
\hline What is Gamification? & 54 \\
\hline 3.3 The Hype Cycle and Critiques of Gamification & 60 \\
\hline 3.4 Case Study: Gamifying the Call Centre & 64 \\
\hline
\end{tabular}




\begin{tabular}{|l|l|}
\hline 3.5 The Theoretical Implications of Gamification & 71 \\
\hline Feedback Mechanisms in Games & 72 \\
\hline The Role of Surveillance & 74 \\
\hline The Quantification of the Self & 77 \\
\hline The Implications of Objective Quantification & 80 \\
\hline 3.6 Conclusion & 83 \\
\hline 4. The Instrumentalization of Play & 85 \\
\hline 4.1 Instrumental Play & 87 \\
\hline 4.2 Miguel Sicart and the Rules of the Game & 92 \\
\hline 4.3 T.L. Taylor and Power Gamers & 96 \\
\hline 4.4 Grimes and Feenberg's Theory of Ludification & 105 \\
\hline Reflexivity & 111 \\
\hline Boundedness & 113 \\
\hline Rule-governedness & 114 \\
\hline Precision & 116 \\
\hline Playfulness & 118 \\
\hline 4.5 Conclusion & 121 \\
\hline 5. Researching Game Development & 123 \\
\hline 5.1 The Paucity of Developer-Centric Research & 123 \\
\hline 5.2 Methodology and The New Spirit of Capitalism & 128 \\
\hline 5.3 Interpretation & 137 \\
\hline 5.4 Conclusion & 141 \\
\hline Part II. The Game Development Landscape & 142 \\
\hline 6. Console Developers: Cogs in a broken machine & 143 \\
\hline 6.1 Core vs Casual & 145 \\
\hline 6.2 Core and the Second Spirit of Capitalism & 148 \\
\hline 6.3 The Rationalization of Console Game Development Processes & 151 \\
\hline Console Manufacturers & 156 \\
\hline Publishers & 159 \\
\hline Developers & 161 \\
\hline 6.4 The Console Life Cycle & 163 \\
\hline
\end{tabular}




\begin{tabular}{|l|l|}
\hline Emerging Phase & 165 \\
\hline Engage Phase & 165 \\
\hline Extract Phase & 166 \\
\hline Decline Phase & 166 \\
\hline 6.5 Conclusion & 167 \\
\hline 7. Maintaining the Machine: Game developers and Core culture & 170 \\
\hline 7.1 Developers and Instrumental Play & 171 \\
\hline 7.2 Core Culture and the Working Conditions of the Power Gamer & 175 \\
\hline 7.3 Core Communities of Practice & 180 \\
\hline 7.4 Conclusion & 185 \\
\hline 8. Blueprints for New Machines: Shifts in game development landscapes & 188 \\
\hline 8.1 Game Industry Sectors & 189 \\
\hline 8.2 Detailing the 2011 Game Development Landscape & 196 \\
\hline Casual & 196 \\
\hline Core & 201 \\
\hline$\quad$ Console & 201 \\
\hline Handhelds & 209 \\
\hline PC - Full Size & 210 \\
\hline MMOs & 213 \\
\hline Indie & 215 \\
\hline 8.3 Situating Industry Shifts as Displacements in the Spirit of Capitalism & 219 \\
\hline 8.4 Conclusion & 223 \\
\hline Part III. Welcome to the Projective Cité & 225 \\
\hline 9. New Economies of Play: Casual, social and mobile games & 226 \\
\hline 9.1 Defining Social Games and their Relationship to Casual and Mobile & 227 \\
\hline 9.2 Funding the Casual Revolution: The Economics of Development & 233 \\
\hline Core Development & 233 \\
\hline Casual Development & 238 \\
\hline The Particular Economics of Social Games & 244 \\
\hline 9.3 Opposing the Revolution: Critiques of social games & 251 \\
\hline 9.4 Responding to Criticisms & 256 \\
\hline
\end{tabular}




\begin{tabular}{|l|l|}
\hline 9.5 Criticism, Tests and the New Spirit of Capitalism & 260 \\
\hline 9.6 Conclusion & 263 \\
\hline 10. Game Design by Numbers & 265 \\
\hline 10.1 Metrics: From Core to Casual & 266 \\
\hline Metrics in Core Sectors & 270 \\
\hline Metrics in Casual Sectors & 273 \\
\hline 10.2 Who Counts in Game Design? Why surveillance and god games matter & 280 \\
\hline 10.3 Innovations to the New Spirit of Capitalism & 287 \\
\hline 10.4 What this Means for Game Design & 293 \\
\hline From Individual to Dividual & 294 \\
\hline Designing Out Designers & 299 \\
\hline Playing the Numbers Game & 303 \\
\hline 10.5 Conclusion & 310 \\
\hline End Game & 312 \\
\hline 11.1 Retracing Our Steps & 312 \\
\hline 11.2 What This Thesis Means for Game Development and Beyond & 317 \\
\hline 11.3 Ways Forward & 319 \\
\hline Appendices & 323 \\
\hline A. Ethics Clearance Form & 323 \\
\hline B. Interview Question Guide & 325 \\
\hline C. List and Description of Online Sources & 328 \\
\hline D. Bibliography of Primary Data Only & 334 \\
\hline Ludology & 355 \\
\hline Bibliography & 359 \\
\hline
\end{tabular}




\section{List of Tables}

2.1 Comparing the 2nd Spirit of Capitalism to the New Spirit of Capitalism 30

8.1 Key sectors of the digital games industry 192

10.1 Quantitative vs qualitative methods of evaluating player behaviour 268

\section{List of Illustrations}

3.1 Gartner hype cycle

4.1 Ludification and the rationalization of games

6.1 Five stages of the digital game industry

6.2 Production stages and increases in staff on a 24-month project

6.3 Profit distributions for a $\$ 60$ game

155

6.4 Game development's actors and interconnections

162

6.5 The variable bargaining power of game developers

8.1 The gaming landscape in 2011

8.2 Global digital games sector revenue (\$B)

8.3 The reach of casual sectors in the U.S.

8.4 Profit distribution for retail $v$ s downloadable games 205

8.5 Integrated console and downloadable game development cycle 208

10.1 "Narrows" Halo 3 heat map

10.2 The shift from web analytics to social analytics 275

10.3 Ninja Metrics and a player's true worth 284

11.1 Data and predictive governance in the game industry

\section{List of Appendices}

\begin{tabular}{|llr|}
\hline A & Ethics Clearance Form & 323 \\
\hline B & Interview Question Guide & 325 \\
\hline C & List and Description of Online Sources & 328 \\
\hline D & Bibliography of Primary Data Only & 334 \\
\hline
\end{tabular}




\section{Chapter 1. Challenges and Change in the Digital Game Industry}

This dissertation uses ground-level accounts from those within the digital games industry to chronicle ideological, technological, and economic changes in the industry, focusing on how games are transforming as play becomes instrumentalized. It pays particular attention to the struggles of developers as they search for creative freedom and autonomy in a risk-averse industry. It makes original contributions to the literature on games by situating and explaining industry-wide shifts in terms of the socio-economics of game development and the rationalities that drive individual developers. It contributes to social theory more generally by explaining how transformations in play, games, and creativity are linked to much wider adaptations in the operation of capitalism and how it is justified to both workers and consumers.

I moved to Montreal in July of 2012, and from my office window could see the offices of Funcom. They had just released The Secret World, a game they had been working on for the last ten years. A few weeks later, amidst positive user reviews, Funcom was forced to lay off over half of their four hundred employees due to "cost adjustment" initiatives (Funcom 2012). My friends at the studio were suddenly, once again, looking for jobs, and those few still employed were wracked with survivors' guilt.

In the games industry, this is not a rare occurrence. From 2009 to 2012, console studios from around the world collapsed completely, including Bizarre Creations, Team Bondi, Black Rock Studio, Hudson Entertainment, Kaos, and numerous THQ Digital Studios including Phoenix, Warrington, and Australia (Dyer 2011; Plunkett 2012). Continued allegations of unpaid overtime, work weeks that exceeded 80 hours, and 
exploitative working conditions plague the industry, despite repeated efforts to improve developers' quality of life (Crossley 2011c, 2011e; Remo 2010a; Sliwinski 2012;

International Game Developers Association 2004). ${ }^{1}$ These troubles extend to full-size PC and $\mathrm{MMO}^{2}$ game studios, including 38 Studios, creators of Kingdoms of Amalur, who spectacularly defaulted on a $\$ 75$ million loan from the state of Rhode Island (Curtis 2012).

In contrast, the mobile and social game sectors of the industry are booming. Market analysts report that revenues for the mobile entertainment industry alone will rise from $\$ 33.2$ billion to nearly $\$ 38.4$ billion in 2011 (Crossley 2011f), spurring fears about the death of the AAA console industry as game industry investors, and developers along with them, move to more hospitable realms (R. Fahey 2011b, 2012).

The digital game industry has fallen. A new games industry has risen in its place. This thesis chronicles the fall of the "Core" sectors of the industry, or what I simply call "Core", situating and explaining these shifts in terms of the socio-economics of game development. Employing social theory, I explain how transformations in play, games, and creativity are linked to wider adaptations in the operation of capitalist systems. Using data gathered from developer interviews, industry conferences, news sites, blogs, and other industry publications, I show how definitions of 'games' and 'play' are changing as they come into contact with technology, allowing games to be reformulated in powerful new ways, so they are not only tools of entertainment but also tools of governance. I

1 A 2010 survey of 2,630 game developers found that, on average, developers spent over thirteen weeks a year in crunch mode (Remo 2010b). This meant that during crunch periods, instead of working a standard 40 hour work week, developers worked, on average, over 55 hours per week. This equates to working the equivalent of about five extra eight-hour, five-day work weeks per calender year.

2 "MMO" refers to a massively multiplayer online game. 
explain how play becomes instrumentalized, highlighting the struggles of developers as they search for creative freedom and autonomy. I ultimately argue that the collective valuation of objective quantitative data and the belief in the fallibility of individual creative autonomy has tumed game design into what I call "design by numbers".

\section{I.1 Industry Upheavals}

Long dominant, the console ${ }^{3}$ sector has become a lumbering dinosaur struggling to survive, while developers on the margins (e.g. Indies) and 'outsiders' (developers of social games, mobile games and what I will discuss shortly as "gamification" services) are experiencing growth and financial success. On their own, developers' accounts of industry upheaval and change are valuable as they chronicle an important phase in the short history of digital games. Details of these upheavals and the conditions that fuel industry-wide change are necessary to understand how new forms of games, forms that are premised on commodification and surveillance, have now become ascendant. Such accounts outline an ongoing battle between business and creativity that is fought within game studios, a battle that also takes place in 21 st-century workplaces more generally, from boardrooms to factory floors. ${ }^{4}$ The depiction of industry upheavals in this thesis describes how economic and production models change as they move further from traditional, hierarchically managed workplaces, to agile networked modes of governance, detailing what these changes mean for both employees and consumers. ${ }^{5}$

3 Consoles refer to the game hardware that primarily is used for playing digital games on a TV. The Atari 2600 , the Nintendo, the Xbox, Playstation 3, and the Wii, are all popular game consoles.

4 The New Spirit of Capitalism promises to end this battle, showing how business concerns can work in parallel with creative endeavours; however, I argue that in the games industry these promises are largely false.

5 These upheavals continue the trajectory of flexible capitalism and lean production which started in the 1970 's, but further extend the reliance on data collection and analysis as a method to predict fickle 
These upheavals can be understood in terms of major trends that are challenging and changing the digital game industry, including:

1) The increasing viability of alternative revenue streams such as advertising and microtransactions, facilitating a shift from marketing games as a retail product towards a more long-term service-based orientation;

2) Changing models of production and distribution that challenge traditional distribution chains, thus lowering the barriers to entry to game development, increasing competition, and driving down game price points;

3) The rapid rise and unanticipated popularity of free-to-play (F2P) Casual games, particularly social games such as those on Facebook, and mobile games such as those played on iPhones; and,

4) The spread of non-game applications that capitalize on the feedback mechanisms and information infrastractures made popular by F2P games, and utilize them in real-world governance projects, hereinafter referred to as "gamification".

All of these trends are intertwined with each other, cross-pollinating and rapidly hybridizing in order to offer games to players at very little cost, while simultaneously turning sites of idle play into sites of economic production. While these upheavals have direct implications for the industry, game developers, and players, I will show how their impact is much broader as innovations developed and honed within the game industry filter outwards to other fields.

This thesis is dedicated to making connections between shifts in the political economy of the game industry, the influence of technological innovations, and my own sociological analyses of developers' day-to-day work. This thesis is bound together by references to the "New Spirit of Capitalism" (Boltanski and Chiapello 2005, 2007). Boltanski and Chiapello explain how capitalism is continually reorganizing itself, emphasizing freedom in order to drive though new, more efficient, work practices and more subtle forms of exploitation. This "Spirit", outlined further in Chapter Two, is

consumer desires and increase efficiency in the workplace. 
important to this thesis because it accounts for the current upheavals in the game industry. Changes to the Spirit of Capitalism have initiated tectonic shifts, reforming the geography of the game industry and creating fissures in the landscape that allow new game sectors to emerge, while others struggle to avoid being buried. In turn, innovations from the game industry, particularly the emphasis on data-driven design, address preexisting weaknesses in the New Spirit of Capitalism, allowing it to operate more successfully.

The Cité is an important concept in The New Spirit of Capitalism and is a recurring topic throughout this thesis. Cités present citizens with different notions of the common good, prescriptions on how to achieve it, and definitions of what is just versus what is unjust. Boltanksi and Chiapello argue that, in the past decades, a new Cité has formed. The Projective Cité is oriented around the network - both in terms of modes of production, but also in terms of envisioning how the world and social relations work. The Projective Cité emphasizes lean enterprises, teamwork and customer satisfaction, and the vision of leaders (not managers) who inspire and mobilize their operatives. The ideal capitalist unit is portrayed as a self-organized team that externalizes its costs onto subcontractors and deals in the production of knowledge rather than the production of industrial goods. The Projective Cité, premised on networked modes of production, assures citizens that this is the ideal configuration of the workplace and that these changes in production serve the common good. This concept will be further explained in the following chapter. Before further introducing the theoretical framework of the thesis and its underlying structure, I will first show why games are an important topic of study, 
provide a brief overview of game studies, and outline the central contributions of the thesis.

\subsection{Why Study Games?}

As summarized by Katie Salen and Eric Zimmerman, games and play in general have many important functions. These include serving as: a release of surplus energy; an expression of general exuberance or joie-de-vivre; an expression of aggression, anxiety or sex drive; youthful practice for adult life skills; a necessary context for exploration and experimentation; a vital means of socialization; and, a tool for self-expression and diversion (2004:309). For the progenitors of game studies, Johan Huizinga (1955) and Roger Caillois (1961), the larger social significance of games lies in the homologies between their structure and social forms, for example, between: "playing house" and keeping house, games of chance and the stock market, or team sports and military endeavours.

Following the work of Clifford Geertz (1973) and Brian Sutton-Smith (1997), Sara Grimes and Andrew Feenberg emphasize that play provides an essential opportunity to "reenact, transgress, and otherwise make sense of larger systems of social order" such as power relations, social hierarchies, etc., even if this reenactment is mostly symbolic (2009:109). This relates to two arguments I make about digital games in this thesis. Firstly, the "free movement" of play is increasingly restricted in digital games, and secondly, this reenactment of power relations and social hierarchies moves from symbolic to actual as games and players are made more productive. 
Digital games are as important and influential as other media such as film and television, but have historically tended to fly under the academic radar. A potential reason for this is that game practices are often conceptualized as in opposition to the "real world" and "real life"; they are seen as virtual simulations that have little impact on outside reality. ${ }^{6}$ This academic radar only 'pings' with prominent recent public arguments that digital games have a negative societal influence, causing violence, fostering addiction, and promoting a retreat from reality (see Jayson 2011; S. Cunningham, Engelstätter, and Ward 2011; Alexander 2010a; Brightman 2011a; Strauss 2011; Orland 2011c; Collins 2011). ${ }^{7}$ However, restricting digital game research to psychological media effects studies on games and violence vastly underestimates and neglects the massive social and cultural importance of games outlined above. Setting games apart from the "real world" understates the significant role they have in our lives. ${ }^{8}$

The growing size and economic impact of the digital game industry is reason alone to study games and their development. Individual games such as Halo 2 have earned phenomenal amounts of money ( $\$ 125$ million on its first day) (Barr, Noble, and

6 Vili Lehdonvirta (2010) shows the preponderance of the term "real world" in game studies articles and argues that game scholarship is based on a dichotomous "real world vs. virtual world" model that elides the real effects of games and the fact that game worlds frequently flow over and into non-game spaces. Most game scholars - even the ones she directly faults - would agree with her.

7 Some may speculate that this lack of attention is due to the relative newness of the game industry (the first commercial game console, the Magnavox Odyssey, was released in 1972), or demographics of game players (the assumption that only young boys are interested in digital games) and thus are unworthy of concerted study by "serious adults". However, I would argue that the relative lack of attention paid to digital games is due to their being classified as idle play, rather than a category of media that has 'real' artistic merit, such as books or cinema. Simply put, research grants for "sitting around and playing games" are a difficult sell. Not surprisingly, searching for a link between school shootings and games is more easily funded.

8 In my own writing, I have also struggled with the term "real world". However, despite its acknowledged flaws (Lehdonvirta 2010), no appropriate substitute has been accepted in game studies terminology. Accordingly, I maintain the use of "game world" and "real world", despite their obvious flaws, and my belief that these worlds actually feed into and bleed into each other. At the very least, the terms are an improvement over the "magic circle" that places play within a bounded space, entirely separated from reality (see Consalvo 2009b). 
Biddle 2007: 281) and US revenues for the industry as a whole have been growing at a double-digit rate. Revenues recently eclipsed those of domestic film box offices, reaching $\$ 12.5$ billion USD in 2007 (Fullerton 2008:xix) and \$15.9 billion USD in 2010 (Entertainment Software Association 2010:10). Game development budgets take on Hollywood proportions. In 2007, the average Xbox360 or Playstation 3 console game budget was in the $\$ 13-30$ million range (Fullerton 2008:423), while a few short years later, development and marketing budgets for games such as Modern Warfare 2 topped \$200 million USD (Nieborg 2011:216). In terms of the commodification of the culture industry, games are a heavy hitter.

We should study games not only because of their economic impact, but also because they are a massively popular leisure-time activity. $72 \%$ of American households play computer or video games (Entertainment Software Association 2010:2). ${ }^{9}$ Youth in the United States spend an average of 20 minutes per day playing digital games, making games the second most popular form of entertainment after television (Fullerton 2008:xix). Contrary to public misconception, the average gamer is not a gawky preteen and teenager, but a thirty-seven year-old adult. In fact, women over the age of eighteen represent a significantly greater proportion of gamers (37\%) than boys seventeen or younger (13\%) (Entertainment Software Association 2010:3). The average age of players increases each year, evidencing that those who grew up playing games are likely to continue to play games throughout their lives.

9 There are multiple reasons to be wary about statistics from the Electronic Software Association (ESA), a lobby and consumer interest group that serves the American game industry. I justify my usage of them here given that the general trends they report are echoed by numerous other sources such as the NPD, Nielsen, Gartner, and EEDAR. More generally, independently verified statistics about the game industry are difficult to find (see Kerr 2006:46). However, the lack of academic statistics and preponderance of corporate ones exemplifies the argument that games are in need of greater academic study. 
Finally, we should study digital games because - despite being historically villanized as promoting antisocial, violent youth - they are now increasingly framed in terms of their positive impact on socialization, education, and health. For example, according to studies from the Electronic Software Association, more than half of parents believe that games provide more physical activity now than five years ago, while over two-thirds of parents believe that gaming provides mental stimulation and education for their children. Games are also seen as a positive social force: $57 \%$ of parents believe that gaming helps their family spend time together, while $54 \%$ of parents believe that games serve a social function, helping their children connect with friends (Entertainment Software Association 2010:5). The role of digital games in our lives will likely increase: as of 2010 , in the US alone, over $90 \%$ of children ages two to seventeen were gaming (approximately 64 million) - an increase of 9 points when compared to 2009 (Radd 2011a). As this thesis will demonstrate, digital games are an increasingly important topic of study and sociological lenses, in particular, can contribute to a deeper understanding of games and their role in each of these domains (economics, play and leisure activities, and socialization).

\subsection{A Brief Overview of Game Studies}

This central intent of this thesis is to examine how the New Spirit of Capitalism operates in the game industry, linking it to current trends in gaming such as the collapse of Core, and the emergence of new game sectors and economic models. I do not intend to map out game studies thoroughly as a field or recount the history of game development in an authoritative manner. Other authors have done this for me (Kline, Dyer-Witheford, and de 
Peuter 2003). Accordingly, rather than providing a very detailed overview of all of game studies, in briefly reviewing game studies literature, I will paint with broad strokes, focusing on those works that have a direct relation to this thesis.

While the majority of popular literature on games is written by gamers and journalists, and despite the tendency of games to fly under the academic radar as noted above, recently the field of game studies is growing and attracting increased scholarly attention. Game studies spreads across many disciplinary divides, including media studies, sociology, anthropology, psychology, human-computer interaction (HCI), engineering, computer science, and economics, to name just a few. This scholarly attention has multiple branches. The first examines the nature of digital games and play. This body of work draws upon broader scholarship on games and play to define what constitutes a game, more generally (Caillois 1961; Huizinga 1955; Avedon and SuttonSmith 1971; Suits 1967), and a digital game, ${ }^{10}$ in particular (Salen and Zimmerman 2004; Juul 2005). Defining games and play promotes the categorization of forms and genres of digital games, as well as provides the groundwork for a more systematic analysis and design of digital games (Juul 2005; Consalvo and Dutton 2006; Salen and Zimmerman 2004). ${ }^{11}$

For the purposes of this thesis, I draw from Bernard Suits and define a "game" as a voluntary attempt to overcome unnecessary obstacles. ${ }^{12}$ To be more precise:

10 Following Kerr (2006), I use the term 'digital games' to encompass PC games, console games, handheld and cell phone games, online games and virtual worlds. In the context of this thesis, it is used interchangeably with 'video games'.

11 A number of textbooks for aspiring game developers help define games and play. For example, see (Crawford 1984, 2003; Fullerton 2008; Schell 2008)

12 Similarly, Jesper Juul defines games according to six necessary and sufficient conditions. Games are: 1) a rule-based formal system; 2 ) with variable and quantifiable outcomes; 3 ) where different outcomes are assigned different values; 4) where the player exerts effort in order to influence the outcome; 5) the 
... to play a game is to achieve a specific state of affairs [prelusory goal], using only means permitted by rules [lusory means], where the rules prohibit use of more efficient in favour of less efficient means [constitutive rules], and where the rules are accepted just because they make possible such activity [lusory attitude]. (1978:41)

Games are intrinsically valued activities, with rules and goals that are voluntarily accepted by players. This does not preclude players from negotiating said rules and goals. Without intentionality on the part of players (i.e. the prelusory goal of wanting to play a game), however, the game ceases to be a game. Play must be entered voluntarily, as an end in itself. For example, if workers were forced to play a pirate game as a condition of their continued employment, their actions would no longer be considered playing a game.

As William Robinson points out, this freedom of choice is a key element of games and play. He uses Mark Twain to illustrate this point:

Mark Twain offered a similar insight into play in his book The Adventures of Tom Sawyer: "If [Tom] had been a great and wise philosopher, like the writer of this book, he would now have comprehended that Work consists of whatever a body is obliged to do, and that Play consists of whatever a body is not obliged to do". (2012:32)

I draw from Salen and Zimmerman to define play (2004). They organize play behaviour into three categories. Game play is the formalized interaction that occurs when players follow the rules of a game and ludic activities are non-game behaviours, such as children tossing a ball in a circle, in which participants are "playing". Thus, game play is a subset of the larger category of ludic activities. Being playful extends even further, encapsulating both game play and ludic activities. It is the state of being in a playful frame of mind, injecting the spirit of play into some other action. Salen and Zimmerman define play

player feels emotionally attached to the outcome; and, 6) the consequences of the activity are optional and negotiable. For an extended discussion on why Suit's definition is preferable to Jesper Juul's more structuralist definition (2005:6-7), please see (W. Robinson 2012). 
itself as "free movement within a more rigid structure" (2004:311). Play emerges both because of and in opposition to more rigid structures, such as rules. ${ }^{13}$

The digital games industry is relatively young. Rooted in the post-World War II military complex, it was only effectively commercialized in the 1970's when companies such as Atari and Magnavox brought consoles into homes. Academic work on the history of the industry often focuses on the console sector and is told from the perspective of network effects and externalities ${ }^{14}$ in the 'console wars' (Schilling 2003; Mateos-Garcia et al. 2010; Shankar and Bayus 2003; D. Williams 2002; Clements and Ohashi 2004; Afuah and Grimaldi 2005). These accounts of each of the seven console generations evidence a processing power arms race, where each new console attempts to outdo its predecessors, first in terms of processing power, marketed in terms of bits, then in terms of processor speed, graphics, bandwidth, and memory size.

The cultural history of console and PC gaming is recounted in book-length accounts written by journalists, including Leonard Herman (1997), J.C. Herz (1997), Steven Poole (2000), Steven Kent (2001), Tristan Donovan (2010), and Harold Goldberg (2011), to name just a few. This body of work includes historical accounts of specific console manufacturers, such as Nintendo (see Sheff 1993) and Microsoft (see Takahashi 2006). In academic circles, Stephen Kline, Nick Dyer-Witheford, and Greig De Peuter provide an extensively detailed history of the game industry from its roots in the military,

13 For example, a play on words such as a riddle or pun exhibits playful behaviour, working within the rules of grammar to carve out a space of play, such as an unexpected punch line. As we will see in Chapter Seven, developers exhibit a playful attitude about their own work, playing within the technological constraints determined by their hardware and software tools.

14 "Network effects" describe how the value of a product or service is dependent on the number of others using it. Accordingly, in the game industry, accounts of network effects emphasize the struggle to attracted the largest market share of players, as well as attract a large stable of developers to publish more and more games. 
through the rise of arcade gaming in the 1970 's, to the growth of the console industry in the 1980's and onwards (Kline et al. 2003).

Beyond the history of digital games, one of the oldest branches of game studies explores the relationship between games and other media. There are two 'sides' to this research: the narratology side which seeks to link digital games to other media such as literature, theatre and film (Aarseth 1997; Murray 1997; Ryan 2004; A.R. Galloway 2006; Brenda Laurel 1991; Bolter and Grusin 1999) and the ludology side which seeks acknowledgement of the unique characteristics of games - especially their formal elements and rules (Juul 2005; Frasca 2001; Bogost 2007). Both sides assess the utility and drawbacks of applying theories and methodologies from other fields to the study of digital games.

Yet another branch of game studies analyses games in the context of learning and literacy (Gee 2007; Squire 2003), and highlights the way in which games cultivate skills and knowledges, such as those suited to the information workforce (Yee 2006b; Dibbell 2006). ${ }^{15}$ It engages in a much larger debate about how games inculcate values and behaviours, the latter of which potentially include both positive and negative behaviours, such as increased problem solving ability on the one hand, and addiction and aggression on the other (Jenkins 1999; S. B. Johnson 2005; Cover 2006). This vein of game studies has attracted the most controversy and media attention. While most often framed in the context of media effects and potentially detrimental impacts of games, there is increasing emphasis on how games might be leveraged to promote real-world change (McGonigal 2011).

15 I include the work on games for health, and 'serious' and 'persuasive' games in this category. 
Another branch of game studies examines the production of digital games using a political economy lens. This work highlights the industry of digital game production, its linkage into a larger "military-entertainment" complex, software labour and development practices (Kline et al. 2003; Dyer-Witheford and de Peuter 2009), and the shift of players from consumers to co-producers that comes with the popularization of end-user content ${ }^{16}$ (Terranova 2000; Kücklich 2005; Humphreys 2005; Sotamaa 2007). Related work on the production and business of digital games comes from many fields, including geography (Johns 2006), sociology (Kerr 2006), political economy (Nieborg 2011), and management and organizational studies (Tschang 2005; Readman and Grantham 2006). This thesis extends and builds upon this branch of game studies.

Despite the richness and breadth of academic game research, there are gaps in the game studies literature. The majority of social science and humanities research is on players and on the content of games. Researchers rarely address the process of making these games. ${ }^{17}$ Questions of why they are made, who makes them, and how they are made go unanswered. The role of developers and their influence on how we play is overlooked. It is part of the 'secret life' of game software that is hidden from players. As designer Brenda Brathwaite argues, a historical understanding of the people behind games is even absent in game development programs:

Similar comparisons could be made to an architecture student who knew nothing of Frank Lloyd Wright, or an art student who could not identify Michelangelo but was totally aware of the majesty of the Sistine Chapel. ...

16 The term "end-user content" is used in the field of game development to refer to content that has been created and produced by players rather than game company employees. Examples include clothes and accessories in Second Life, mods in World of Warcraft and complete levels in Little Big World.

17 Ethnographies of game developers are rare (Banks 2009; Malaby 2009; O'Donnell 2008), but can be supplemented by a number of first-person accounts from industry insiders. For example, see (Morningstar and Farmer 1991; B. Laurel 2001; Koster 2002; Bartle 2004; Stone 1995). 
However, I regularly encounter graduates of game programs who know nothing of game history. They can talk about Final Fantasy, DOOM, the Sims, Age of Empires, and WoW, and acknowledge them as foundational in some way, yet they know nothing of the key minds behind these same games. (2011c)

While it is important to study developers in terms of their influence on cultural production, the need to study the secret life of software goes even further.

This secret life is what goes on behind the scenes - the labour of countless people that is hidden within game discs and digital files and shapes how players learn, play, and behave. It includes the social practices that influence video games' construction and operation, and the political, economic, and social conditions that are embedded in their design and implementation. Because of the significant influence of games in our lives, this secret life should be the focus of increased academic scrutiny. If this is not enough reason to study games, developers are not just interesting for their own sake, but present cases to learn about broader social trends and issues, such as how capitalist ideologies shape the production of technology and workplace organization, thus harnessing worker creativity more tightly than ever before.

The 'secret life' of game software should no longer be kept secret. Software in general, and games in particular, play subtle roles in governing users. Yet they are a black box. We live significant portions of our lives "inside" games, apps, and websites. Software shapes, constrains, and configures our everyday lives, from our language, to our socializing, to our employment and beyond. ${ }^{18}$ It only makes sense to study how this

18 I take a parallel approach to platform studies, which uses game code as a primary site of analysis to investigate how the hardware and software constraints of game platforms shape creative expression (Montfort and Bogost 2009; Jones and Thiruvathukal 2012; Maher 2012). In comparison, I use the discourses of game developers as a primary site of analysis to investigate how socio-economic constraints shape creative expression. 
software is created, the various forces (technological, social, economic) that shape what games do, and the visionaries - as well as drudge workers - that bring games to life.

This thesis follows the political economy branch of game studies, providing industry overviews of the digital game landscape, and explaining how economic constraints, market pressures, and labour concerns influence the forms of digital play. Political economy accounts take a macro approach in their analyses, focusing on histories of the industry, statistical accounts of its growth, demographic analyses of player populations, and executive reports of profits and future corporate trajectories. This thesis diverges from political economy approaches by intertwining the macro-level analysis with the micro-level narratives from 'ground zero' of the digital games industry developers themselves. I argue that digital games, like any other technology, are socially constructed artefacts that emerge from a complex process of negotiation between various human and non-human actors. ${ }^{19}$ Thus, digital games cannot be understood without attention to the economic systems from which they emerge and the political, social and cultural contexts in which they are produced and consumed. Accordingly, while the game industry cannot be understood as separate from its economic context, it also cannot be understood without attention to the social actors that give it shape and the cultural forms that it produces and operates within. Moreover, understanding current shifts in the game industry can tell us about wider shifts in economy and society, particularly in relation to the New Spirit of Capitalism.

19 Two key elements make games unique from other technologies. Firstly, they are voluntary. For example, unlike word processors, players do not need games to accomplish a task. They are there to be entertained. Secondly, unlike other entertainment media, games are directly interactive. Unlike film, the game depends on the player's input. Balancing these two elements (voluntary and interactive) means that developers carry a much heavier burden than both software developers and media producers, in terms of predicting what players will do and keeping players continually engaged with the system. 


\subsection{Thesis Overview}

Broadly, this thesis is about economic shifts in the game industry, starting with an overview of the traditional modes of production in Core game sectors, and then moving towards new and emerging Casual games sectors, such as social and mobile games. I argue that shifts in the game industry, particularly the unpredicted rise and success of Casual games since 2009, can be explained by the New Spirit of Capitalism. In turn, an examination of how the New Spirit of Capitalism operates in the game industry shows how modes of capitalism have adapted in ways much different from that predicted by Boltanski and Chiapello.

\subsubsection{Part I. Theorizing and Researching Game Development}

\section{Chapter Two: The New Spirit of Capitalism in the Game Industry}

Chapter Two provides an overview of The New Spirit of Capitalism. I outline the arguments of Boltanski and Chiapello (2007) who, in turn, draw upon Max Weber to describe how, at different historical periods, there are different "Spirits" that moralize and legitimize capitalist endeavours. Each "Spirit" is a configuration of a plurality of Cités, which present citizens with different notions of the common good, prescriptions on how to achieve it, and definitions of what is just versus what is unjust. I outline the emergence of the Projective Cité and the decline of the Industrial Cité, showing how they apply to contemporary shifts in the game industry. I then critique various aspects of Boltanski and Chiapello's formation of the New Spirit of Capitalism, but show how these critiques may 
be addressed by evolving the theory in order to provide a more nuanced account of the game industry than that provided by Dyer-Witheford and de Peuter (2009).

\section{Chapter Three: Games, Gamification, and Governance}

Chapter Three jumps forward, in a sense, to show how the industry upheavals documented in this thesis have impacted the wider world outside of games. I argue that gamification is a paradigmatic example of the New Spirit of Capitalism, wherein game spaces become sites of governance, all time is productive, and all work is play. To link governance projects to the New Spirit of Capitalism, I draw on Foucauldian governmentality studies, particularly Nikolas Rose's work on governing through freedom (1999). Gamification governs through freedom, developing the subjectivities of an idealized networked citizen who, even in their leisure time, is focused on selfimprovement. Play becomes an instrument of governance, a tool to master and reshape one's self, rather than an end in itself.

In this chapter, I first define the concept of "gamification", showing how the analytics practices honed by the game industry are applied to non-game online contexts. I argue that the data collected from playing the 'game' is used to encourage changes in real-world behaviour and a playful care of the self based on quantitative metrics and automated feedback practices. Gamification measures are applied to a wide arrangement of activities, from self-improvement projects, to education, to marketing, to financial management. Yet, as demonstrated with the case study I analyse in the last half of the chapter, the values these gamification processes represent remain the same, rooted in instrumentalized play and further promoting, competition, advancement, accumulation, 
and efficiency.

\section{Chapter Four: The Instrumentalization of Play}

If play is supposed to be inconsequential, how did we get to gamification and games as tools of governance? The following chapter explains the history of governance and games and the roots of gamification, outlining the ideological preconditions that must exist for gamification to exist. It traces changing notions of play, and the ideological shifts in how games are thought about that allows the New Spirit of Capitalism to influence leisure activities, leaving little room for the (unproductive) play of old.

I begin the chapter by relating the instrumentalization of play to larger rationalization and instrumentalization processes, noting how these processes take a specific form in the game industry. I then provide a review of how "instrumental play" has been defined by game studies scholars. My analysis draws from three notions of instrumental play: instrumental play as first introduced by T.L. Taylor $(2003,2006 b)$, and then later reworked by Miguel Sicart (2010), and the instrumentalization and rationalization of play (aka the "ludification theory") proposed by Sara Grimes and Andrew Feenberg (2009). I detail how play changes as it becomes rationalized, and in turn, how playful moods, game mechanics, narrative devices and the aesthetics of games are increasingly applied to everyday governance. While the preceeding chapters take a largely player-centric view, the remaining chapters of the thesis focus on developers, and provide more in-depth detail about industry-wide shifts. 


\section{Chapter Five: Researching Game Development}

This chapter further outlines the paucity of developer-centric research, highlighting the need to research game development in much greater depth, exposing the heterogeneity of the industry and the complex roles of developers. This call for research is often stymied by the difficulty in gaining access to what is an intensely secretive high-tech industry. Accordingly, the remainder of the chapter focuses on my methods for both data collection and data analysis, explaining how I tracked and charted industry changes over time and interpreted these changes. I make comparisons to the methodology of Boltanski and Chiapello $(2007,2005)$, drawing parallels to their work, but also highlighting certain dissimilarities. In particular, instead of relying on management literature, my data sources pay particular attention to the "great men" and voices of the industry who work to define and solidify the justificatory regimes of developers, defining which innovations to embrace versus which to resist.

\subsubsection{Part II: The Game Development Landscape}

In Part II of this thesis, I argue that in order to understand the instrumentalization of play, what this means for both developers and players, and how this relates to the New Spirit of Capitalism, we must first understand how game development works, particularly the economic influences that effectively determine what forms of games get made, how they get made, and who makes them. Accordingly, the next chapters provide an overview of the game industry. To understand the massive shifts in the game industry, we must travel to the epicentre of the industry: Core console development. 


\section{Chapter Six: Console Developers: Cogs in a broken machine}

In this chapter, I further define the divide between Core and Casual game sectors, linking Core development to the Second Spirit of Capitalism, and hierarchical production models. I provide a brief overview of the console sector, reviewing how console games are made, the role of developers in making games, and the instrumental pressures that shape what forms of games are ultimately published, making brief parallels to other culture industries such as film. Primarily using economic analysis, I explain why the console industry is contracting in the face of massive upheavals. Developers are the creative force behind games - conceptualizing, designing, and coding our spaces of play. But in the larger chain of console development they are a small cog in a rationalized production cycle, occupying a subservient position in contrast to publishers and manufacturers and bargaining from an undesirable negotiating position. In this sense, Console development epitomizes the (outmoded) Industrial Cité.

\section{Chapter Seven: Maintaining the Machine: Game developers and Core culture}

This chapter provides the human counterpart to the preceding socio-economically focused chapter. Instead of presenting developers as exploited dupes, or victims of technological determinism, I highlight how they take active roles in maintaining and propagating the industry that often exploits them. While Chapter Four detailed playercentric accounts of instrumental play, this chapter starts with the work of Casey O'Donnell, showing how developers themselves - much like power gamers - embrace an 
instrumental play of their own. I highlight the role of Core culture, emphasizing how developer communities of practice take an active role in resisting change. Despite this resistance, the increasing rationalization of creative production and the constraints imposed by publishers and console manufacturers are pressuring developers to explore new modes of development. While developers may not be victims of technological determinism, the forms of games they make are shaped by economic concerns. New modes of development are framed in alignment with the New Spirit of Capitalism, promoting efficiency and productivity as well as autonomy.

\section{Chapter Eight: Blueprints for New Machines: Shifting landscapes of game development}

Panning out from the Console epicentre, Chapter Eight examines how the larger industry is evolving from the landscape depicted by Aphra Kerr's 2006 industry overview in response to the pressures of console production. The chapter provides a "lay-of-the-land" of the larger game industry constellation, introducing and defining two polarized territories of game development: Core sectors, comprised of Console, PC, MMO and indie games, and Casual sectors, comprised of casual, social, and mobile games. Fuelled by the affordances of online connectivity, this chapter explores the adaptation of Core sectors in response to digital distribution, and details the emergence and rapid growth of the Casual sectors due to alternative revenue models that alleviate the economic constraints currently epitomizing Core production. I situate these shifts within the Core sectors as displacements in the Spirit of Capitalism. The New Spirit of Capitalism 
innovates with critique, revitalizing traditional sectors. Ultimately, the territorial divides between Core and Casual are blurring as each side mimics and adapts successful strategies from their competitors.

\subsubsection{Part III: Welcome to the Projective Cité}

In order to mitigate the financial risk of Core game production, ever more constraints are placed upon developers until routes for creativity - experimentation and iteration in particular - are stifled. Facing these constraints, the lack of creative freedom, and the accompanying conditions that follow, Core developers look for escape routes, abandoning the Industrial Cité for the Projective Cité. However, as I argue in Part III, these escape routes come with constraints of their own. And, because the Projective Cité seems to address many of the critiques of the Console sector (unwieldly hierarchies, restrictive management, lack of agility), developers have no ground left to stand on in terms of resistance.

\section{Chapter Nine: New Economies of Play: Casual, social and mobile games}

Chapter Nine is a case study of social games, comparing how they address many of the artistic critiques of Core developers. I compare the traditional economic models of console development with the new revenue streams utilized by social games, highlighting the prevailing socio-economic conditions that spur revolutionary shifts towards Casual games. Hoping to escape the constraints of the Core sectors, developers have flocked to their development. 
While promising developers more freedom, games from these emerging sectors are actually closely tied with the instrumentalization of play, both in terms of users' play, but also in terms of the play of developers themselves. Play and creativity are increasingly quantified and put to instrumental purposes that are predicated upon feedback and closely monitoring and channelling players' actions and developers' iterative design responses in order to reduce the financial risk inherent in game development. By determining what players spend time and money on, developers rationalize production and avoid wasting resources on other assets or mechanics. Ideally, this reduces financial risk and allows developers to experiment with new and innovative game ideas. In practice, however, I show how gameplay and game development in emerging sectors is highly scripted and constrained.

The chapter draws on work from the New Spirit of Capitalism to show how capital adapts and responds to criticism, fostering new work practices and more successful and subtle forms of exploitation. As game development focuses increasingly on selling an online service rather than a one-off game, the work of developers becomes increasingly rationalized, and play more closely parallels the modes of productivity detailed in Chapter Three. Creating games, much like playing them, becomes a numbers game.

\section{Chapter Ten: Game Design by Numbers}

Chapter Ten investigates how one particular technology, metrics, has transformed game development in terms of what game design looks like, how developers exercise their 
creative autonomy, and how human subjects are conceptualized and governed. I show how the adaption of data management technologies and predictive governance projects have wide-ranging impacts on the Projective Cité that extend far beyond the games industry. Because metrics address many of the drawbacks of the original New Spirit of Capitalism, they are an ideal case study to example how capitalism rapidly adapts.

Instead of creative production remaining the domain of game designers, game development becomes directed by quantitative analytics. This reliance on numbers reintroduces the rationalization that was rejected by the Second Spirit of Capitalism. Because metrics come from the bottom-up (i.e. are gleaned from player data) and not the management hierarchy, they are less open to critique. When this is coupled with the industry-wide valuation of objective data over subjective feedback (on the part of players, developers and publishers alike), there is increasingly less space to critique this more extensive and pervasive form of rationalization. Accordingly, the play spaces that should ostensibly be free from rationalization and capitalism, exhibit a level of level of commodification and control that is unprecedented.

\section{Chapter Eleven: End Game}

Chapter Eleven ties together the key arguments of each chapter into a broad narrative of change in the digital game industry. It highlights the central takeaways for those interested in applying the lessons of the game industry more broadly, emphasizing datadriven design as a corrective to the failures of the Projective Cité, and highlighting how capitalism is strengthened in the face of critique. The chapters in this thesis offer an 
explanation of the unpredicted shifts in game development, shedding light on the influence of metrics in revitalizing the industry, but also pointing out the potential negative impacts data-driven design has for creative autonomy and human subjectivity. I conclude with a few thoughts about the future of game development. 
Part I.

Theorizing and Researching Game Development 


\section{Chapter 2. The New Spirit of Capitalism in the Game Industry}

The notion of the New Spirit of Capitalism is the glue that binds each of the following

thesis chapters together, providing a framework of analysis that shows how current events

in the game industry are tied to much larger socio-economic trends. In The New Spirit of

Capitalism Luc Boltanski and Eve Chiapello $(2007,2005)$ examine working conditions

that are now endemic in the game industry: the rise of contract jobs, precarious labour, and rising profits for corporations. They define capitalism as the imperative to unlimited

accumulation of capital by formally peaceful means. The basis mark of capitalism is

reintroducing capital into the economic circuit with a view to deriving profit (2007:4).

They note that, despite fewer jobs and the lack of job security, we have trouble critiquing

capitalism and instead are fatalistic. ${ }^{20}$ They use a comparison of French management

literature from two time periods - the 1960 s and the 1990 s - to "open the black box of the

last thirty years", showing how forms of both capitalism and critique evolve over time,

thus giving readers insights into how to formulate more effective critiques of capitalism

(2007:xliv).

Ideology $\mathrm{y}^{21}$ plays a large role in their work. Following Weber, they call the

20 To define this 'fatalism', they contrast the strikes and worker unrest in France in the 1960s and 1970s, compared to their relative paucity today. Fatalism, for Boltanski and Chiapello is defined by the lack of widespread organized resistance to capitalism. Acute issues, such as AIDS, homelessness, etc. are addressed as individual 'causes', but not the larger, systemic framework of capitalism that creates the conditions that give rise to these causes. In many ways, this echos Zygmunt Bauman's arguments about "Liquid Modernity", and how the emphasis on individualism and the lack of community acts to the detriment of larger society (2000).

21 This ideology is defined as a set of shared beliefs, inscribed in institutions, bound up actions, and hence anchored with reality (much like Foucauldian "rationalities"). The "spirit" of ideology and material conditions do interact, but not in a teleological manner. As Boltanski and Chiapello emphasize, this is not ideology in the Marxist sense (i.e. a moralizing discourse that is intended to conceal material interests) (2007:3). Boltanksi and Chiapello give substantial weight to the beliefs and justifications of actors themselves. In this sense, ideology is not a mask that veils power relations and exploitation. Ideology is derived from the norms and actions of individuals themselves, and it changes in response to individuals' critiques. 
ideology that justifies engagement in capitalism, the "spirit of capitalism" (Weber 2002; Boltanski and Chiapello 2007). People require strong moral reasons to justify capitalism, both on an individual level (i.e. when a person finds grounds for engaging in capitalist enterprise) and on a social level (i.e. a belief that engagement in capitalist enterprise serves the greater good). The Spirit of Capitalism moralizes and legitimizes capitalist endeavours, but it also constrains accumulation by providing justification and setting limits on what counts as 'fair'. Actors internalize the Spirit of Capitalism, thus constraining their individual actions in the real world. In short, the Spirit of Capitalism tells us the '"right' actions to be performed to make a profit and the legitimacy of the actions" (Boltanski and Chiapello 2007:16). Accordingly, the Spirit of Capitalism also tells us what is 'wrong' and open to critique. Critiques of capitalism are thus made in the name of values that are deemed to have been betrayed by the capitalistic process (i.e. when certain actions step outside the bounds of what is deemed 'fair' by the Spirit of Capitalism, such as price fixing and monopolies in a free market economy).

\subsection{Shifts in the Spirit of Capitalism}

Boltanski and Chiapello argue that there are different Spirits of Capitalism and different underlying values at work at different periods of time. Weber's Protestant Ethic (2002) details the first Spirit of Capitalism, while Boltanski and Chiapello detail two further shifts: one in the 1930's-1960's that took place as small family-owned businesses morphed into larger hierarchical corporations; and, another in the 1980's and 1990's as these corporations decentralized into lean and agile networked companies. Both of these 
shifts exemplify different Spirits of Capitalism and change what is considered fair work.

Each Spirit of Capitalism comes with new forms of accumulation and

organization, as well as new sets of problems and solutions, which are depicted in Table

1.1, below. These shifts are grounded in different justifications, premised on three key

elements; 1) what is exciting about this New Spirit? What new freedoms does it offer?;

2) What forms of security are offered for those involved and their children?; and, 3) How

is this New Spirit fair? How does it cohere with a sense of justice, and thus contribute to

the common good?

Table 2.1: Comparing the Second Spirit of Capitalism to the New Spirit of Capitalism

\begin{tabular}{|c|c|c|}
\hline & $\begin{array}{l}\text { Second Spirit of Capitalism } \\
(1940-1970)\end{array}$ & $\begin{array}{l}\text { New Spirit of Capitalism } \\
\text { (Since 1980s) }\end{array}$ \\
\hline $\begin{array}{l}\text { Forms of the capital } \\
\text { accumulation } \\
\text { process }\end{array}$ & $\begin{array}{l}\text { Managerial firms } \\
\text { Big industrial companies } \\
\text { Mass Production }\end{array}$ & $\begin{array}{l}\text { Network Firms } \\
\text { Internet and Biotech } \\
\text { Varying and differentiated } \\
\text { production }\end{array}$ \\
\hline $\begin{array}{l}\text { The Problems that } \\
\text { have been identified } \\
\text { by Boltanski \& } \\
\text { Chiapello }\end{array}$ & $\begin{array}{l}\text { Unsatisfied "managers" } \\
\text { (excessively technical roles } \\
\text { that lack in autonomy; } \\
\text { criticisms of company } \\
\text { directors' power; decrials of } \\
\text { the negative side effects of } \\
\text { bureaucracy) } \\
\text { Managerial problems } \\
\text { associated with firms' gigantic } \\
\text { size (the threat of bureaucracy } \\
\text { for liberty) }\end{array}$ & $\begin{array}{l}\text { Rejection of hierarchy as a form } \\
\text { of domination } \\
\text { "Liberation" of all wage-earners } \\
\text { (and not only managers as was } \\
\text { the case during the } 1960 \text { s) } \\
\text { Competition and permanent } \\
\text { change }\end{array}$ \\
\hline The Solutions & $\begin{array}{l}\text { Decentralization } \\
\text { Meritocracy } \\
\text { Management by objectives }\end{array}$ & $\begin{array}{l}\text { Lean companies, organised in } \\
\text { networks or by projects; } \\
\text { reengineering; flexible, } \\
\text { innovative and competent } \\
\text { organizations }\end{array}$ \\
\hline
\end{tabular}




\begin{tabular}{|c|c|c|}
\hline & $\begin{array}{l}\text { Competent } \text { cadres }^{22} \text { and } \\
\text { executives, selected on the } \\
\text { basis of personal merits } \\
\text { demonstrated during an entire } \\
\text { career spent working for a } \\
\text { single firm. }\end{array}$ & $\begin{array}{l}\text { (capable of managing people } \\
\text { who are autonomous and } \\
\text { creative) } \\
\text { Coaches, managers, experts } \\
\text { Self control, control by the } \\
\text { client, trust }\end{array}$ \\
\hline & Hierarchical Control & \\
\hline $\begin{array}{l}\text { What is being } \\
\text { rejected }\end{array}$ & $\begin{array}{l}\text { The private sphere (personal } \\
\text { judgements, nepotism, } \\
\text { promotions through loyalty as } \\
\text { opposed to merit, allegiances } \\
\text { and privileges) }\end{array}$ & $\begin{array}{l}\text { Cadres } \\
\text { Bureaucracy } \\
\text { Separation between one's private } \\
\text { and professional lives }\end{array}$ \\
\hline
\end{tabular}

Source: (adapted from Boltanski and Chiapello 2005)

Boltanski and Chiapello argue that within the New Spirit of Capitalism, a new model of the firm has emerged that is premised upon network metaphors, an organization that is "very flexible; organized by projects; works in a network; features very few hierarchical levels; where a logic of transversal flows have replaced a more hierarchical one, etc." (2005:165). This new firm directly contrasts with the idealized firm of the second Spirit of Capitalism, which focused on "hierarchical organization, integrated and geared towards the internal realisation of activities (vertical integration)" (2005:165). The game industry today evidences both of these organizational forms. The console sector, especially manufacturers and publishers, are organized in a hierarchical manner, while emerging sectors emphasize the rapid change and agility predicated by the network model.

22 The French term for managers. Cadres were university educated employees and during the Second Spirit of Capitalism replaced the family in terms of running organizations. They emphasized long-term planning, rationalization, and economies of scale. Cadres were employed by shareholders, evidencing a break: the people who "owned" the organization were no longer those who were "running" it. 


\subsection{Defining the Cité}

Each Spirit of Capitalism depends on different social orders - sets of intertwined value systems and norms. To differentiate these social orders, Boltanski and Chiapello rely on the theoretical construct of the Cité. The Cité is an important concept in The New Spirit of Capitalism. Financial success is not enough to justify adherence to the capitalist system, especially as most citizens have little chance of profit. What is needed are justifications that link personal gains from involvement in and acceptance of the system, to some notion of the common good. This is what Cités provide. Cités present citizens with different notions of the common good, prescriptions on how to achieve it, and definitions of what is just versus what is unjust.

Cités co-exist with each other. At different times, under different Spirits, and in different locations and workplaces, different Cités are dominant. Each capitalist spirit is a ideological configuration composed of a plurality of smaller Cités. Boltanski and Chiapello (2007) define a number of different justificatory regimes (Inspirational, Domestic, Renown, Civic, Market, Industrial), showing how each Cité relies on different value systems to evaluate whether a distribution of goods was done in a fair and legitimate matter, how to measure a person's worth, how the common good is served, etc. For example, in the Inspirational Cité, an individual's worth rests on their creativity, artistic sense, authenticity. In the Market Cité, greatness resides in those who offer highly coveted goods in a competitive marketplace, while in the Industrial Cité, greatness is based on efficiency and professional abilities. Modern societies are not a single social order/Cité, but co-exist in the same social space. However, at certain times, specific Cités 
are dominant.

Boltanski and Chiapello used management texts from the 1960 s and 1990s to determine what Cités were ascendant during each time period. They detail how firms after the 1960s reorganized their production process and wage contracts, and note the rising popularity of flexible labour systems, sub-contracting, team-working, multi-tasking and multi-skilling, and the emphasis 'flat' management. These changes are widely labelled as "lean capitalism" and "post-Fordism", and work is often characterized in terms of being part of a flexible network of production.

Boltanski and Chiapello identify a new Cité that characterizes the New Spirit of Capitalism, one that is composed around the network as a harmonious figure of natural order. ${ }^{23}$ Boltanski and Chiapello argue post-Fordist trends evidence the creation of a new Cité, one between the Market and Industrial Cités. The Projective Cité emphasizes lean enterprises, team-work and customer satisfaction, and the vision of leaders (not managers) who inspire and mobilize their operatives. The ideal capitalist unit is portrayed as a self-organized team that externalizes its costs onto sub-contractors, and deals in the production of knowledge rather than the production of industrial goods. The Projective Cité, premised on networked modes of production, assures citizens that this is the ideal configuration of the workplace, and that these changes in production serve the common good.

Boltanksi and Chiapello compare this new Project-ive Cité to the Industrial Cité prominent during the Second Spirit of Capitalism (and still evident today). In the

23 Each Cité carries its own ontology in terms of being composed around a harmonious figure of natural order that conveys ideal-types in the Weberian sense. For example, the ideal-type of the Projective Cite is the network, while the free market is the ideal-type of the Commercial Cité. 
Projective Cité, people are evaluated in terms of their activity, mobility, flexibility, adaptability, and their ability to form new and diverse network connections with others. In the Industrial Cité people are evaluated in terms of their productivity, efficiency, loyalty and place in the workplace hierarchy. The Industrial Cité is premised on stability and long-term job security. The Projective Cité is premised on short-term projects where participants develop new skills and network links, thus opening the door to new projects once the existing one ends. Projects are not restricted simply to work, but encompass all activities, thus blurring the separation between work and play (e.g. past-times of modding and leading a MMO guild develop skillsets that can enhance one's workplace success). The Projective Cité characterizes innovation in the game industry, while the Industrial Cité represents all that it is struggling to leave behind.

\subsection{The Role of Criticism and Adaptations to Capitalism}

Boltanski and Chiapello are not simply interested in describing the New Spirit of Capitalism. They are interested in the transformations in these Spirits of Capitalism which render critique ineffective. In other words, they are interested in explaining why the move to a New Spirit of Capitalism is accompanied by a lack of critique and a lack of resistance (i.e. Why do youth today accept the lack of jobs and the rising qualifications that are required to gain access to even temporary entry-level positions? or, to frame this in terms of the game industry: Why are so many opponents of social games now creating them?). In particular, they want to explain why historical forms of critique, especially those exemplified by the protests of May 1968 in France, have lost their power. ${ }^{24}$

24 Boltanski and Chiapello separate these critiques into two camps: artistic (qualitative) critiques, and 
Their answer is two-fold: capitalism survives by endogenising critiques, absorbing and adapting in response to them. Critiques thus play a dual role: they shape capitalism into less-objective forms, but in doing so, they also strengthen capitalism. More importantly, critique is silenced when the underlying value systems that justify capitalist change (i.e. when we shift from a Industrial Cité to a Projective Cité), as critical activities have no established foundations in this new, unfamiliar moral ground. For example, if developers collectively value the mobility and flexibility to set their own hours and regularly change their occupations in favour of those that creatively challenge us, then any critiques centred on the lack of job security fall flat. Importantly, Boltanski and Chiapello show how the critiques of capitalism and the Industrial Cité prominent in France during May 1968 were effectively enrolled into the capitalism system, helping it evolve and adapt into the New Spirit of Capitalism and the networked forms of business we see today. They argue that in order to revive critique, we must root it in the value systems of Projective Cité, embracing the ontology and epistemology of the Projective Cité, and thus speaking in the language of networks to improve existing networked ways of work rather than rejecting them altogether.

\subsection{Critiquing the New Spirit of Capitalism}

The book The New Spirit of Capitalism (2007) is not without faults. For example, academic critics such as Sebastian Budgen (2000) point to issues with Boltanski and

social (quantitative) critiques. Social critiques highlight capitalism as a source of poverty and inequality. Capitalism is depicted as a source of opportunism and egoism which encourages private interests, thus destroying social bonds and collective solidarity, and widening the gap between the rich and the poor. Artistic critiques, in contrast, highlight capitalism as a source of disenchantment and inauthenticity. The capitalist market is an impersonal, dominating force directly opposed to the freedom, autonomy and creativity of humans (Boltanski and Chiapello 2007:37). 
Chiapello's methodology. Boltanski and Chiapello examine management texts as a key site where the symbolic order of the New Spirit of Capitalism is reproduced. However, they use a relatively small sample and provide little proof that Projective ideals are transmitted from the management executives who read the books down to the workers they manage. I return in Chapter Five to these critiques of Boltanski and Chiapello's work, outlining alterations to my own research methodology that address these criticisms.

In the preface to the English version of The New Spirit of Capitalism (2007:xxi) Boltanski and Chiapello refer to other critiques that problematize their scope of study: The New Spirit of Capitalism limits its analysis to the geographical confines of France and overemphasizes the influence of May 1968. My thesis addresses these critiques by examining how the New Spirit of Capitalism can be applied to domains outside of Europe and workplaces far different from the unionized factory floors that Boltanski and Chiapello describe. In regards to May 1968, I use it as a touchstone that is emblematic of a critique of capitalism that was not simply confined to the geographic borders of France, but was much more widely dispersed. Even to those outside of France, May 1968 resonates.

In addition, I have my own further critiques of The New Spirit of Capitalism, and I address each of them in this thesis. Contrary to the promises of the Projective Cité, the work of developers seems less autonomous and creative now than it was in the 1970s. The freedom of innovation promised by the Projective Cité has not borne fruit. Much of the motivation for my own work lies in examining how creativity is effectively constrained in digital game sectors, even ones that embrace the Projective Cité. 
Boltanski and Chiapello's vision of the Projective Cité relies heavily on the existence of visionary leaders to guide networks of workers. But beyond a small number of mythic figures such as Steve Jobs, visionaries seem to be largely absent from our systems of production. There are simply not enough "great men"25 to guide the system, and there are far too many network exploiters who selfishly put their own success ahead of the health of the network and the well being of their co-workers. Moreover, the ideal of "great men" who are willing to make sacrifices for the benefit of others seems counterintuitive to the networked individualism the Projective Cité promotes. I deal with this issue directly in Chapter Ten. Finally, Boltanski and Chiapello ignore the role of technology in propagating and maintaining capitalist systems. This is a key contribution of my own thesis, examining how technological change and innovations such as data driven design work to re-shape the Projective Cité and the New Spirit of Capitalism.

In contrast to The New Spirit of Capitalism, Games of Empire (Dyer-Witheford and de Peuter 2009), has also analysed the political economy of the game industry, providing an alternate - yet often complementary - theoretical approach. Nick DyerWitheford and Greig de Peuter frame the history and structure of the digital games using a critical social theory lens, arguing that digital games are the paradigmatic media of Empire (Hardt and Negri 2001). "Empire" is a planetary, militarized, hypercapitalism that is reliant on immaterial labour ${ }^{26}$ and cognitive capitalism ${ }^{27}$. Dyer-Witheford and de Peuter

25 "Great men" is Boltanski and Chiapello's term, not mine.

26 Immaterial labour refers to work involving information and communication, particularly the labour that produces the informational, cultural, or affective element of the commodity (Dyer-Witheford and de Peuter 2009:xx)

27 Cognitive capitalism refers to systems of production in which knowledge plays the integral role. Arising in the 1970s, it is a new configuration of capitalism that is defined by its dependance on immaterial labour and the transformation of knowledge into a commodity (Dyer-Witheford and de Peuter 2009:37). 
show how Empire structures the industry and becomes a thematic trope of games themselves. They also outline the multitudes of indie developers, political games, and rebellious counter-gamers that oppose Empire through the development of alternative worlds to play in and alternative regimes of political organization to structure them. The strength of Games of Empire is that it links the production of games, the analysis of specific games, and players themselves, together via immaterial labour, their respective roles in Empire, and cognitive capitalism. Games of Empire is a carefully researched accounting of the political economy of the Core game industry (i.e. Console and PC sectors) that emphasizes the often forgotten role of material production, such as the third-world countries that mine the materials for console manufacturing and the factory workers that assemble these consoles.

Like myself, Dyer-Witheford and de Peuter examine how Capital permeates everyday life - not just the lives of developers but also the lives of player-consumers. This Capital is flexible and adaptable in the face of critique. Discourses of "fun", "games", and "play" are used to obscure other relations of domination and exploitation. However, Dyer-Witheford and de Peuter only examine the Core industry, and do not address recent trends such as F2P, microtransations, social network games, mobile games, gamification, and the decline of retail. They represent Core sectors as ideal versions of post-Fordist capitalism. Accordingly, they do not (or cannot) address the current collapse of Core sectors and the precariousness of the industry. In contrast, by analysing the industry a few short years after Dyer-Witheford and de Peuter, I am able to document the rapid adaptations of capital as it responds to critique. 
A central difference between this thesis and Games of Empire lies in our theoretical influences. Hardt and Negri, and by extension, Dyer-Witheford and de Peuter draw from Marx, while Boltanski and Chiapello draw from Weber. This necessitates a different view of ideology, a different view of agency, and thus a different methodology. Those drawing from Marx view ideology as a mask or delusion that shields actors from the reality of capitalist oppression, crisis or decay. Weberian understandings of ideology, in contrast, give more weight to the beliefs and justifications of actors themselves, rather than consigning them to categories of false consciousness. Actors willingly enroll in different Spirits of Capitalism because these Spirits align with their views of justice and the greater good.

Dyer-Witheford and de Peuter seek to uncover the forces and laws within the structures of the game industry that elude the actors they study. Consequently, they do not prioritize the voices of those they write about. In contrast, I look to the actors themselves, focusing on game developers' depictions of industry change and their shifting notions of what is 'right' and 'good' and how they define 'success' in the game industry. I treat their concerns as valid, and do not see them as dupes of the system. ${ }^{28} \mathrm{I}$ focus on the context of developers in order to provide a ground-level account of industry change. ${ }^{29}$

28 I discuss this further in Chapter Seven.

29 Another difference between this thesis and Games of Empire is how "networks" are conceptualized. The network of Empire is everywhere, encapsulating everything. There is no outside, thus the only way to resist Empire is through the embedded multitudes that reshape the network from within. Boltanski and Chiapello's understanding of network operates on a different level. They take into account the underlying power of network-based thinking, tracing its roots to the work of Social Network Analysts, Latour, Deleuze and beyond. They pay attention to how concepts of networks get enrolled into capitalism and filtered through management tests, to create the justifications and values of the Projective Cité. Boltanski and Chiapello have a much more nuanced view of the "network", and a recognition of how the language of networks is a powerful tool of capitalist systems. Accordingly, appropriating and reshaping this language via alterations to the Projective Cité is the route to critique and resistance. 


\subsection{Applying the New Spirit of Capitalism to the Game Industry}

This thesis uses the New Spirit of Capitalism as a framework to more broadly situate and explain the current shifts in the game industry. In particular, the struggles of the console industry and the rapid emergence of social and mobile game sectors can be located in terms of Core sectors - premised on the second Spirit of Capitalism - attempting to compete with Casual sectors, whose structure and operation embodies the New Spirit of Capitalism. Core vs Casual is thus reframed in terms of industrial vs network modes of capitalism.

In addition to using the New Spirit of Capitalism to situate shifts in the game industry, I use the game industry to explain how the New Spirit operates on the ground. In particular, I use examples of the Casual Revolution (i.e. the rise of social and mobile games) to examine how the New Spirit absorbs and adapts to critique. I also highlight how it legitimizes itself in terms of specific rationalities premised on self-governance, freedom, and autonomy. My contribution to the body of scholarship influenced by the work of Boltanski and Chiapello is to highlight how current instantiations of the New Spirit of Capitalism and the Projective Cité diverge substantially from their model first outlined in 1999. In particular, I show how the Projective Cité is reformulated and strengthened by innovations in metrics and "Big Data" (massive data sets), that allow corporations to gather user information for the purpose of "crowdsourcing". This quantitative data reveals the emergent "wisdom of the crowds", and objectively points the way forward for industry, without the need to rely on fallible individual visionaries - a key figure in Boltanski and Chiapello's model. This effectively turns the production of 
culture into "game design by numbers". ${ }^{30}$

Broadly, this thesis is about economic shifts in the game industry, starting with an overview of the traditional modes of production in Core game sectors, and then moving towards new and emerging Casual games sectors. I argue that shifts in the game industry, particularly the unpredicted rise and success of Casual games since 2009, can be explained by the New Spirit of Capitalism, and the shift in emphasis from a social orders where the Industrial Cité is dominant, to one wherein the Projective Cité is rapidly taking over.

Core sectors such as console development are in decline because they still rely on hierarchical work structures (despite claims that they are completely post-Fordist). In contrast, Casual sectors, such as mobile and social games, promise more initiative and autonomy in the workplace, that is enabled by a network-based form of organization. In short, the Core is collapsing because it cannot promise the creative freedom that is idealized by the Projective Cité. Core development is characterized by long development cycles, big budgets, big talent, and bleeding edge technologies. Excessive constraints hamper creativity, including massive unwieldly teams, tight schedules, long work hours, a risk averse management, a hit-driven emphasis that restricts new genres, costly hardware, middleware, certification processes, and a commitment to the retail distribution of a static product.

Developers of Casual games lack many of the overwhelming constraints

30 My understanding of game design by numbers and the use of metrics is inspired by the Quantification of the Self movement endorsed by Wired magazine technorati such as Kevin Kelly and Gary Wolf (see www.quantifiedself.com). However, parallels can be drawn to the work of a number of other authors, for example, on the history of statistics and the valuation of quantitative knowledge (see Hacking 1990; Poovey 1998; Porter 1996), as well as those authors that problematize contemporary statistical practices (see Best 2001; Haggerty 2001). 
(financial, scheduling, hierarchical oversight) faced by Core developers. Importantly,

Casual games are drawing resources from outside of the games industry: attracting players who do not consider themselves gamers, drawing funding from investors who would never invest in traditional development, and attracting developers who have roots outside of games. But upon closer analysis, these sectors are even more tightly rationalized and constrained than traditional development.

The following questions propel this thesis, and focus on charting and untangling the socioeconomic impacts of industry-wide changes to how games are developed:

- What does the future of game development look like? How is it different from traditional models of Core console development?

- Do emerging models offer developers a respite from the constraints and tensions characterized by console development?

- How do these emerging models work? How are they profitable?

- Do these models offer more freedom, play, or power to developers?

- What do these new models of production mean for the shape of games and the experience of players?

- How is the instrumentalization of play enhanced by these emerging trends? and,

- How are games enrolled in governance, in terms of the governance of developers and their creative work, but also the governance of players via playful rhetorics? 


\section{Chapter 3. Games, Gamification, and Governance}

It could be argued that a chapter on gamification has no place in a thesis on the digital games industry. Gamification efforts have been vociferously critiqued and rejected by game developers, most gamification services operate outside of the game industry proper, and the success rate of gamified projects is spotty to say the least. However, the rise of gamification exemplifies how notions of games and play are increasingly leveraged in non-game spaces. Gamification aligns with the New Spirit of Capitalism and the network-centric values of the Projective Cité, thus fuelling its success in the face of critique. Gamification governs through freedom, developing the subjectivities of an idealized networked citizen who, even in their leisure time, is focused on selfimprovement. Discourses of gamification promise to make work more like play, and to make education, healthy living, and mundane tasks 'fun'. In this sense, digital games have come a long way from Space Wars and Solitaire. Games are productive. They are essential tools for self-governing subjects. This chapter charts the rise of gamification, using a case study of a call centre to show how gamification presents an idealized version of the New Spirit of Capitalism. In order to do so, I start by discussing the ideal subject in the Projective Cité and tie this subjectivity to Foucauldian notions of governing through freedom.

\subsection{The New Spirit of Capitalism and Governing Through Freedom}

Using the New Spirit of Capitalism (2007) as a reference point, the rapid rise of gamification can be tied to the protests and strikes of May 1968 in France and similar 
social movements elsewhere in Europe and North America. In short, gamification provides an ideal route towards the autonomous self-governance deemed necessary for success in the New Spirit of Capitalism. According to Boltanski and Chiapello, the unrest of the 1960's started within the ranks of blue collar workers, then spread to students and academia. Critique centred on the alienation created by factory models of industrial production $^{31}$ : the disenchantment of everyday life, the dehumanization of the world under technology, and the cold militaristic rationalizations embedded in large corporate hierarchies. Dominant themes included a "denunciation of 'hierarchical power', paternalism, authoritarianism, compulsory work schedules, prescribed tasks, the Taylorist separation between design and execution, and more generally, the division of labour" (Boltanski and Chiapello 2007:170). These critiques of labour were paralleled by calls for domestic emancipation and bundled with demands for "autonomy and self-management, and the promise of an unbounded liberation of human creativity" (Boltanski and Chiapello 2007:170). In short, both blue collar workers and university students rebelled against being cogs in a vast machine, and fought for increased autonomy and creativity in the workplace.

Forty years later - after the collapse of the American automobile industry and the dot.com bubble - capital has absorbed the critique of the 1960's, and the models of workplace organization are vastly different. Hierarchical workplaces are seen as inflexible and unable to keep pace with rapid technological change. Corporations are now organized on a network model, centred on lean cores comprised of largely autonomous

31 These 'artistic critiques' were coupled with social critiques. However, the social critiques fell from prominence early on as unions successfully bargained for wage increases. Alienation, in contrast, was much less amenable to simple solutions on the part of organizations. 
personnel oriented around specific projects. These cores are surrounded by a constellation of support services - temps, contractors, outsourcing, subcontractors, etc. - that can be cut away or added to at will depending on the company's needs. Instead of compulsory work schedules and prescribed tasks, work hours are increasingly flexible and individuals change careers multiple times in their lifetimes. Instead of long-term job security, freedom of movement is epitomized. For example, in the software industry, periodic moves every few years are the norm as people leave for new challenges rather than 'stagnate' in the same job. Rather than climbing the rungs of the organizational ladder, networking, personal initiative, and self-development are held up as the keys to workplace success. ${ }^{32}$ In the face of critique, the Second Spirit of Capitalism has succumbed to the New Spirit of Capitalism, and - in terms of the underlying values, justificatory regimes, and norms espoused by its citizens - the Industrial Cité has made way for the Projective Cité.

The ideal worker in the second Spirit of Capitalism is the career man. ${ }^{33}$ Loyal to the company and focused on increasing efficiency and productivity, the career man spends his life working for a single firm, improving his position due to the merits of his work and his ability to meet predefined company objectives. In contrast, the ideal worker in the New Spirit of Capitalism feeds on permanent change, valuing mobility and the

32 As I argue in Chapter Five, the symbolic work involved in broadcasting the norms and values of the Projective Cité happens in many places. For Boltanski and Chiapello (2007) much of this work is done though management texts and the business leaders who promote networked modes of capitalism. For the game industry, this symbolic work is achieved via developer conferences, news sites, and other publications and web sites that serve as a mouthpiece and sounding board for those in the game industry.

33 This reference to "career man" points to the gendering of the workplace in the 1960's. While one might make links to the comparative feminisation of the networked workplace, that is tangential to the aims of this chapter. 
challenges of each new workplace. They move from project to project. Each project further develops their employability, thus increasing opportunities for future projects. Companies provide self-help resources, but the ideal worker is one who manages their self and leverages their personal life experiences and social networks to fuel innovation and creativity in the workplace. Autonomy and self-governance are key.

At this point, it is an easy step to tie changing working conditions within the game industry to the shift from the Industrial Cité to the Projective Cité, a topic to which I return later in the thesis. But first I want to make broader argument - one that applies not just to makers of games, but also to players - games are becoming tools to motivate and train us. ${ }^{34}$ Not just in the game industry, and not just in the workplace, but in everyday life. The foundation for this argument rests on a complementary theoretical approach to NCS: governmentality ${ }^{35}$, specifically that of governing through freedom.

Governmentality scholars such as Nikolas Rose (1999) highlight the role of technology in the emergence of new decentralized control strategies and the reconfiguration of old strategies. This has particular resonance in describing the specific control strategies that are appearing in games. First introduced by Michel Foucault (1991), the term "governmentality" refers to the organized practices (mentalities, rationalities, and techniques) through which subjects are governed. ${ }^{36}$ Governmentality

34 This argument has been made elsewhere by a number of game scholars (Squire and Jenkins 2004; Gee 2007; Kline, Dyer-Witheford, and de Peuter 2003). Certainly, games have been used in classrooms as instructional tools for decades.

35 I am using governmentality as a toolkit to draw from, rather than an unyielding and predefined framework.

36 Elsewhere in game studies, the broader work of Foucault has used to discuss conflict management in games and virtual worlds (MacKinnon 1997), the 'work' of power gamers (Silverman and B. Simon 2009), the creation of productive player populations (Kücklich 2009), and lateral surveillance between players (Taylor 2006a). 
scholars define their problem space in terms of "the conduct of conduct". This government includes all endeavours to "shape, guide, direct the conduct of others ... And it also embraces the ways in which one might be urged and educated to bridle one's own passions, to control one's own instincts, to govern oneself" ( $\mathrm{N}$. Rose 1999:3). It is here that the link between games and the self-governing subject begins.

Part of the utility of governmentality approaches is the recognition that governance ${ }^{37}$ is conducted not just in a hierarchical top-down manner by the nation-state but by myriad other actors in many different locales, consequently broadening the scope of study to include new spaces of governance, such as games and online spaces, and new methods of governance that are subtle and less recognizable than the overt laws, policies and regulations that have been prioritized in other studies of government. While not commonly seen in this light, recognizing how games are technologies of government highlights how strategies of governance take new forms in spaces created by technology. It emphasizes how techniques to constrain and control people in these spaces may be unfamiliar in comparison to the laws or norms to which we are commonly accustomed, and draws attention to the way that technical innovations change the way we interact with each other and are consequently governed.

Governmentality studies, particularly Nikolas Rose's work on Powers of Freedom (1999), align with the work of Boltanski and Chiapello (2007) $\cdot^{38}$ Both are influenced by

37 Governance refers to any "strategy, tactic, process, procedure or programme for controlling, regulating, shaping, mastering or exercising authority over others in a nation, organization or locality" (N. Rose 1999:15).

38 Given the part of May 1968 in the academic trajectory of Foucault, and thus governmentality, this is perhaps not surprising. The protesting cadres of the 1960's became experts in Foucaldian critiques of power, especially critiques that rejected authoritarianism and tyrants in favour of the extraordinary potential of the individual and personal relationships. They employed this knowledge in in their future professions and consultancies, bringing about the New Spirit of Capitalism, and thus proving that 
network metaphors and the work of Bruno Latour. As acknowledged by Rose, current

shifts in governance are tied to the rise of networked organizational structures:

[R]ecent political strategies have attempted to govern neither through centrally controlled bureaucracies (hierarchies) nor through competitive interactions between producers and consumers (markets), but through selforganizing networks. Politics is seen as increasingly involving exchanges and relations amongst a range of public, private and voluntary organizations, without clear sovereign authority. Terms like 'actor networks', 'self regulatory mechanisms', 'trust', 'habits and conventions', 'gift relations' and 'informal obligations' are utilized to describe the actual operation of the complex exchanges through which governance occurs. (1999:17)

Boltanski and Chiapello organize the governance strategies and tactics associated with self-organizing networks under one banner - that of the Projective Cité and the New Spirit of Capitalism.

Activities of government are inextricably bound up with activities of thought. In the language of governmentality, Cités (i.e. justificatory regimes) are rationalities. Rationalities, like Cités, have distinctive moral forms, "embodying conceptions of the nature and scope of legitimate authority ... and the ideals that should guide the exercise of authority: freedom, justice, equality, responsibility, citizenship, autonomy and the like" (N. Rose 1999:26). They have distinctive languages and ideologies, as well as distinctive problem spaces and solutions. They are regimes of $\operatorname{truth}^{39}$ : coherent systems of thought rooted in specific historical contexts and structuring what may be thought, who are the authorized knowers, and what are the responsibilities of citizens. These regimes of truth/Cités thus change over time. In the Projective Cité, governing through freedom ${ }^{40}$ is

critique can never really claim victory, but rather simply transforms capitalism.

39 Rose argues that 'truth' changes over time, in the sense that certain "languages of description, explanation, calculation and judgement came to acquire the value of truth and the kinds of actions and techniques that were made possible by such truths" (1999:8).

40 Governing through freedom is a key element in other Cités as well, given that definitions of 'freedom' change over time (e.g. sexual freedom, freedom of identity, freedom of individual choice, free 
paramount. Following Rose, notions of freedom, with the associated celebration of the

powers of the individual, of autonomy and choice, now underpin attempts to specify and construct new forms of social arrangements (1999:64).

In a Cité rooted in governing autonomous subjects through freedom rather than

hierarchical oppression, the responsibilities of the citizen lie in self-governance.

Individuals self-interestedly govern themselves through introspection, foresight, and

calculation. The New Spirit of Capitalism thus breaks with the hierarchical modes of

control of the Second Spirit, assimilating the demands for autonomy and responsibility

that were before seen as subversive. ${ }^{41}$ Boltanski and Chiapello describe the resultant shift

in the workplace:

To oversimplify, we can say that this change consisted in substituting selfcontrol for control and, consequently, in externalizing the very high costs of control by shifting the burden from organizations on to wage-earners. A proven capacity for autonomy and responsibility constituted one of the new tests that made it possible to part simultaneously with oppositional workers and abusive petty tyrants, whom the new method of control, relying mainly on self-control, henceforth rendered redundant. (2007:191)

The shift from hierarchy to autonomy requires new technologies of government ${ }^{42}$ that shape human conduct in alignment with the rationalities of the Projective Cité. The

markets...etc.). In the Projective Cité freedom rests in individual self determination and consumer choice.

41 Here, it seems, Rose and Boltanski and Chiapello disagree. Rose links current trends in self-governance to neoliberalism (i.e. responsibilization is key in decentralization endeavours premised on leaving the state to steer the boat while the private sector rows), while Boltanski and Chiapello argue that this selfgovernance is not due to neoliberalism but rather a rejection of the oppressive state and its monopoly on violence. In other words, the motivation for self-govemance in the New Spirit of Capitalism is rooted in emancipation, the free association of creators, and a rejection of state government, rather than a belief that the state can function better if it is decentralized (2007: 202). This difference of opinion, however, does not negate my argument, although I tend to side with Rose.

42 A technology of government is "an assemblage of forms of practical knowledge, with models of perception, practices of calculation, vocabularies, types of authority, forms of judgement, architectural forms, human capacities, non-human objects and devices, inscription techniques and so forth, traversed and transected by aspirations to achieve certain outcomes in terms of the conduct of the governed" ( $N$. Rose 1999:53). Games and gamification are technologies of government. 
technologies of government of the Industrial Cité, particularly those such as scientific management and Taylorism, are no longer applicable. In the Projective Cité, the self becomes an object of knowledge and autonomy is achieved through continuous selfimprovement via rational knowledge and technique. Citizens must determine their existence through actions of choice (particularly consumption). In the remainder of this chapter we will see how games and gamification become technologies of government, and how gamification is also a process of translation, forging alignments between the objectives of authorities wishing to govern and the personal projects of those individuals and groups that are the subjects of government, thus tying myriad micro-level gamification projects into macro level governance projects.

\subsection{Gamification}

Social game designer Scott Jon Siegel defines game design as "the imposition of rules onto the chaos of play, in order to elicit a desired behaviour" (2009). However, something entirely different is occurring with the trend of gamification. Every weekday morning, I sit down at my computer. After checking my emails, I head to a gamification website, 750words.com, that promises to help make writing a daily habit by rewarding me with badges, positive feedback, and semantic and statistical analysis about my written text. After I complete my daily quota, I proceed to the task management application on my iPod, Epic Win. Framed as a questing RPG, every task that I complete levels up my avatar and has the chance of uncovering virtual loot. Literally, within an hour of waking up, I am playing at least two applications that promise to help me become a more 
productive worker and prolific writer. Defining these activities as the "imposition of rules onto the chaos of play" is not such a good fit anymore. Gamification turns Siegel's definition on its head. Gamification, in fact, can be defined as the imposition of play onto the rules of non-game activities, in order to elicit a desired behaviour. ${ }^{43}$

The rise of gamification as a concept and an increasingly united movement evidences how discourses of playful behaviour (e.g. fun, exploration, and competition) and game mechanics (e.g. levelling up, leader-boards, and variable reward schedules) are increasingly leveraged to influence people's activities and routines, thus making the examination of play an important avenue of study. Digital games and gamification are ideal technologies of government because - unlike board games - they allow for the compilation of statistics and internal states, and rules to be recorded, thus hiding many of the internal workings from the players and allowing for increased complexity in games. This digitalization makes it much easier to collect player data, and then, as a process of function creep, use this data in new and innovative ways. In the case of gamification, the data collected from playing the game is used to encourage changes in real world behaviour in a variety of ways, some slightly negative, such as targeted marketing, while others are more benign, such as the use of games and associated data visualization techniques to motivate players to write more, lose weight, or develop specific skills. Gamification brings quantification into the domain of our private lives, encouraging a semi-playful accounting of the self. Domains of life that were previously free from quantification (e.g. measuring our popularity, our social aptitude, our reputations, and our

43 To clarify, I am not arguing that all gamification efforts are games, nor am I arguing that gamification is always play (It certainly is not play, if it is not voluntary). While gamification proponents actively link games and play with gamification in terms of their marketing discourses, the actual services themselves may have little similarity to games, other than a points systems or leaderboard. 
individual eating and exercise habits) are now being precisely calculated and quantified. To understand how gamification is related to broader governance projects in the Projective Cité and how games and play are increasingly enrolled in governance, we must first trace the history of gamification and its relationship with the digital games industry.

\subsubsection{The Emergence of Gamification}

In 2010, the gamification movement emerged, uniting a host of pre-existing practices and services under the single banner of "gamification". Epitomized by online technologies such as Nike +, Mint, and Foursquare that pledged to make everyday tasks such as exercising, financial planning and socializing more enjoyable, gamification proponents, fuelled by the successes of social games, promised to make everyday life more like a game. Web services such as Bunchball, Badgeville, Gamify, and Big Door media promised to help 'gamify' websites, a number of books highlighted how gamification practices could be leveraged as a business tool (Edery and Mollick 2009; Reeves and Read 2009; Zichermann and Linder 2010), and a showcase 'Gamification Summit' was announced in January 2011, aiming to unite gamification developers and business leaders who were interested in gamifying their services. The summit's list of speakers included a 'who's who' of gamification evangelists, but also notable game designers such as Jane McGonigal and Amy Jo Kim.

Outside of business/marketing circles, public attention had been drawn to the concept of gamification by Jesse Schell's February 18, 2010 DICE (Design Innovate Create Entertain) keynote address on "Design Outside the Box: Beyond Facebook" 
(2010a). Schell's dystopian talk highlighted the link between the game mechanics of Facebook games, and how these same compulsive mechanics can be applied to everyday activities such as brushing one's teeth to buying consumer goods. In brief, Schell painted a vision of the "Gamepocalypse" wherein users' day to day behaviours would be shaped by points systems and awards for brand loyalty. Picked up by media sources across the web $^{44}$, Schell's vision fuelled discussions on current potentials to "gamify" life, and incited debate about whether the spread of these points based systems heralded playful utopias or dystopic surveillant societies run by corporations and advertisers.

Schell not only increased public awareness of gamification, he also brought the concept to the attention of the mainstream game industry. Heretofore, gamification and the Gamepocalypse were relatively unheard of. But the widely broadcast talks (on YouTube, TED TV, Fora TV, G4, etc.) provided an initial focus for game industry editorials and opinion articles (R. Fahey 2010; Koster 2010; Della Rocca 2010), and then, a few months later, fuelled a more critical examination of the more apparent failures of gamification (Deterding 2010a; Robertson 2010; Alexander 2010d). The gamification movement has, from its onset, been championed by those peripheral to, or entirely outside of, the game development industry. As with the rise of Facebook and iPhone games, early entrants to the field were not designers who had honed their skills creating console games, but were those with backgrounds in web design, business management, and marketing who evangelized gamification and thus defined the field.

44 In April the talk reached a larger audience when it was highlighted on TED TV's "best of the web" (Schell 2010b), and in July Schell reprised his talk for the Long Now Foundation (Schell 2010c). 


\subsubsection{What is Gamification?}

The term "gamification" is a neologism, coined by marketing professionals and used increasingly by those in game development (R. Fahey 2010; Koster 2010). The precise meaning of gamification depends on who is defining the term and what are their underlying institutional affiliations. In overly simplistic terms, game developers and designers define gamification in terms of applying game mechanics, technology, and development techniques to domains outside of games, while those from outside of the industry generally equate gamification with narrower marketing applications that rely on adding points, leader-boards and badges to non-game activities. For example, GamesIndustry.biz columnist Rob Fahey describes gamification as:

... the integration of video game mechanics into non-gaming tasks - the steady "gamification" of the world around us, as more and more actions in our daily lives come to be governed and (perhaps arguably) enhanced by interactions, rules and systems learned from the world of games. (2010)

For industry consultant and past chair of the International Game Developers Association (IGDA), Jason Della Rocca, this process utilizes video game design and systems-based thinking to make a variety of tasks such as fuel efficiency and scientific problem solving more "playful and engaging" (2010). For David Helgason, CEO of the popular game development platform Unity, gamification is directly tied to the use of game development technologies to create non-game applications, such as using game engines to create biomedical models and architectural visualizations (Huling 2010). These industry definitions emphasize how game technologies, such as engines and mechanics, can be utilized in non-gaming activities.

"Gamification" has a slightly different emphasis when described by the marketing 
and advertising professionals who first realized the power of gamification to shape human behaviour (especially consumption) and thus became the self-appointed 'experts' of the movement. When promoting the gamification of business practices ${ }^{45}$ they speak in terms of shaping user behaviours. As stated by Fortune columnist J. P. Mangalindan:

With "gamification," companies study and identify natural human tendencies and employ game-like mechanisms to give customers a sense that they're having fun while working towards a rewards-based goal. In doing so, they hope the added value will enable and reinforce positive behavioural change across a wide spectrum of non-game-related issues - healthcare, finance, philanthropy, general lifestyle. (2010)

While all definitions emphasize the allure of porting ${ }^{46}$ games to real-world processes, marketing-based definitions focus on using behavioural economics to influence user behaviour, such as improving workplace productivity (Reeves and Read 2009) or increasing consumption and brand loyalty (Schwab 2010).

For the purposes of this thesis, I will refer to gamification as defined by Wikipedia, recognizing the meaning of this term is contested and still in flux. ${ }^{47}$ Behavioural economics and marketing references dominated early versions of the definition (e.g. September 2010) which was flagged for a potential bias towards advertising and self promotion (Wikipedia 2010a). The definition underwent substantial deletions and revisions in order to remove the flagged status (Wikipedia 2010b). The fact

45 Notable influences in these fields include companies selling gamification services such as Bunchball and Ooga Labs, as well as individuals such as Stanford Professor Byron Reeves (Reeves and Read 2009) and Gabe Zichermann, public speaker and co-author of Games-Based Marketing (Zichermann and Linder 2010) and Gamification By Design (Zichermann and C. Cunningham 2011).

46 'Porting' is the process of adapting software so that a program can be used in a different computing environment than the one for which it was originally designed.

47 While the use of Wikipedia is often maligned in academic studies (Head and Eisenberg 2010), research has shown that the veracity of Wikipedia entries is comparable to the "gold standard" of the Encyclopedia Britannica (Giles 2005), and exhibits a "hyper currency" in defining new concepts (Head and Eisenberg 2010) as well as demonstrating an admirable transparency in correcting flaws or bias (Benkler 2006:73). 
that the emerging frontiers of gamification are being colonized mainly by behavioural psychologists and marketers, as evidenced by their shaping of the term, is certainly something to keep in mind. Following Geoffrey Bowker and Susan Leigh Star, this Wikipedia definition and affiliation towards marketing ties gamification into a particular infrastructure, "a set of work practices, beliefs, narratives, and organizational routines" that is entangled with a certain set of ideologies (1999:319). These ideologies are expressly rooted in governing through freedom and the values of the Projective Cité.

"Gamification", according to Wikipedia, is:

The use of game techniques and mechanics to enhance non-games. Typically gamification applies to non-game applications and processes, in order to encourage people to adopt them, or to influence how they are used. Gamification works by making technology more engaging, by encouraging users to engage in desired behaviours, by showing a path to mastery and autonomy, and by taking advantage of humans' psychological predisposition to engage in gaming. The technique can encourage people to perform chores that they ordinarily consider boring, such as completing surveys, shopping, filling out tax forms, or reading web sites. (Wikipedia 2012)

While I follow the trend of the majority and call this movement "gamification" (Koster 2010; Huling 2010; Schwab 2010), others have alternately described this process as part of a "metagame" (A. J. Kim 2009, 2010) or "game layer" on top of the world (Priebatsch 2010), marketed it as a form of "total engagement" (Reeves and Read 2009) or "funware" (Zichermann and Linder 2010), and even referred to it as the coming of the "Gamepocalypse" (Schell 2010a). Regardless of preferred label, the uniting idea is that game mechanics and techniques can be applied to non-game processes in order to make them more engaging and fun.

Gamification practices are diverse and wide ranging, including, to name a few: 
1. Car dashboards that use mini-games and graphic visual feedback to reward reduced fuel consumption (Toyota Prius, Honda Insight);

2. Software that allows users to set, track, and achieve their financial management goals (Mint by Intuit);

3. News sites like Slashdot and The Escapist that reward users who post interesting comments with reputation points and recognition;

4. Devices and applications that allow athletes to plan their workouts and runs, select motivational soundtracks, and track incremental improvements and progress while broadcasting their successes to friends on Facebook (Nike +, Adidas miCoach, FitBit);

5. Programs that promote healthy eating habits (healthmonth.com) and a raft of fitness and weight loss 'coaches' for game consoles like the PS3 Move, Xbox 360 Kinect, and Nintendo's Wii and DS;

6. Check-in services that broadcast a users' location to friends and reward brand loyalty with special discounts (foursquare, SCVNGR, Gowalla, Facebook locations);

7. Programs that inspire and reward one to write daily (750words.com) or learn new languages (Rosetta Stone);

8. Mini-games that harness 'click-work' and crowd-sourcing to complete laborious tasks like tagging images in Google (ESP game/ Google Image Labeller), researching about the structure of proteins (foldit) or identify craters on Mars (NASA click-workers);

9. Game-like virtual environments for call centre workers to help inspire teamwork and increase worker productivity; and,

10. iPhone apps for task management that frame 'to-do' lists in terms of an RPG questing game (Epic Win).

Examples of gamification range along multiple continuum: some are marketed as games (e.g. the console games and Epic Win); while others only have tangential links to the video game proper (e.g. the points awarded to website commenters, or the game-like visuals employed in the financial management software). Gamification experiences can be single player (e.g. fuel consumption games in cars), but are more often social experiences, comparing users' results to that of other participants (750words.com) or pitting them in direct competition with each other (foldit). Most gamification practices are linked to one's social graph, allowing achievements to be broadcast to friends, and challenges issued to recruit new users. All of these instances promise to make mundane 
tasks more like a game, and thus more fun ${ }^{48}$ But beyond that, it is difficult to find commonalities that are present in all cases. In fact, many of these examples predate the term "gamification", and are only retroactively compared to and associated with gamification.

The individual practices that comprise gamification efforts are not new, nor is their application to non-game activities. The achievement badges, leader-boards, and levels found in gamification all have well-known non-game equivalents, from classroom grades to boy scout badges. Systems for inciting and rewarding desirable behaviour are also commonplace, from happy hour drink specials to airline loyalty points. ${ }^{49}$ Utilizing games to make everyday tasks more enjoyable and to motivate certain behaviours is also a longstanding tradition, as competitions, challenges, and quests in the form of games predate historical accounts. As argued by Rob Fahey, our "affinity for games predates recorded history - it flows naturally from the competitive, challenge-focused nature of our psychology, which millennia ago we learned to harness in order to make dull tasks tolerable by turning them into games" (2010). As the history of organized sports (Campbell 2000) and board games (Flanagan 2009) attest, using games as a form of training for non-game tasks, such as military conquests to domestic prowess, is certainly

48 I am not arguing here that these gamified services are actually fun. The vast majority are not. However, the gamification movement has co-opted the idea that games are fun and used this to promote their services. The assumption that games = fun is itself problematic, partly because it is so hard to define "fun" (see Koster 2005). It is also quite clear that not all games are meant to be fun. See for example, The Artist is Present, developed by Pippin Barr, where the player waits hours in real-time in a virtual line to attend a virtual Marina Abramovic performance art exhibit.

49 A key difference between contemporary gamification and traditional incentivization is the increasing emphasis on earning status and virtual rewards rather than physical goods. The reduction in engagement costs when marketers shift from incentivization to gamification is driven by leveraging users' competitive natures and drive for social and cultural capital rather than economic capital. Additionally, the novelty of gamification addresses advertising fatigue, offering new ways to attract consumer's attention, as well as the additional ability to collect data on the consumer while doing so. 
not novel.

While gamification practices themselves are not new, what is new is society's understanding and appreciation of how games shape human behaviour. Fahey argues that this understanding, coupled with technological innovation, has initiated a " greater understanding of what makes games compelling, and the toolset required to build that compulsion into more and more tasks" (2010). Simply put, deeper understandings of behavioural economics and psychological motivators of human behaviours allow gamification designers to more successfully leverage games and reward systems to promote desired behaviours. Following Rose, this deeper psychological understanding allows marketers to play upon aspects of human personality that are not rational, making these behaviours intelligible through empirical methods (1999:85). For example, attitude surveys, analysis of spending habits and $\mathbf{a} / \mathrm{b}$ testing are all used to divide the population into more manageable population segments, each with different tastes, weaknesses and motivations that must be targeted differently in order to induce behavioural change.

While the rise of gamification is enabled by a better understanding of human motivation, it is further propelled by technological developments that make it relatively cheap and easy to create a "game layer" on top of many of our daily activities. Part of this ease is rooted in the efficient and ubiquitous collection of information. Our actions are not anonymous online, rather they are collected by websites, and voluntarily shared through status updates, Facebook walls, blog posts, photo sharing services, and Twitter feeds, as well as GPS devices in phones that continually broadcast our location and thus enable a host of location based services and games. This broadcasting of the self provides 
an endless stream of data about our activities that undergird gamification efforts. It is not difficult to create game-like feedback systems that analyze these data flows and tell users, in real-time, how they are doing, thus indicating what they need to do next in order to achieve their goals. Accordingly, businesses such as Bunchball can tweak non-game spaces with relative ease, adding game-like elements such as vibrant settings, points systems, leader-boards, narratives and quests. Preexisting social graphs such as Facebook, MySpace and Twitter are leveraged to turn a single player game (or more accurately, "gamification experience") into a more compelling multi-player competition.

\subsection{The Hype Cycle and Critiques of Gamification}

The gamification movement faces substantial critique. Like most emerging technologies, gamification follows Gartner's Hype Cycle (pictured below), fuelled by high initial expectations and then losing momentum as the products fail to live up to these expectations (Fenn and Raskino 2008). Already, critics occupy a "trough of disillusionment" with gamification. The success of gamification efforts can be quantified and measured by tracking how long and how often users play (i.e. engagement), as well as what they specifically do (i.e. participation). The underlying assumption is that if users are engaged and participating (both measurable concepts), then they must be having fun (a much more difficult concept to quantify and measure). While initial reports of gamification emphasize increased 'play' in terms of traffic on gamified websites, long term measurements of participation are not nearly as positive. Critical reports emphasize that these projects may be compelling on a short term basis, but are in no way 'fun', thus 
limiting their long term allure (Deterding 2011).

\section{Illustration 3.1: Garter hype cycle}

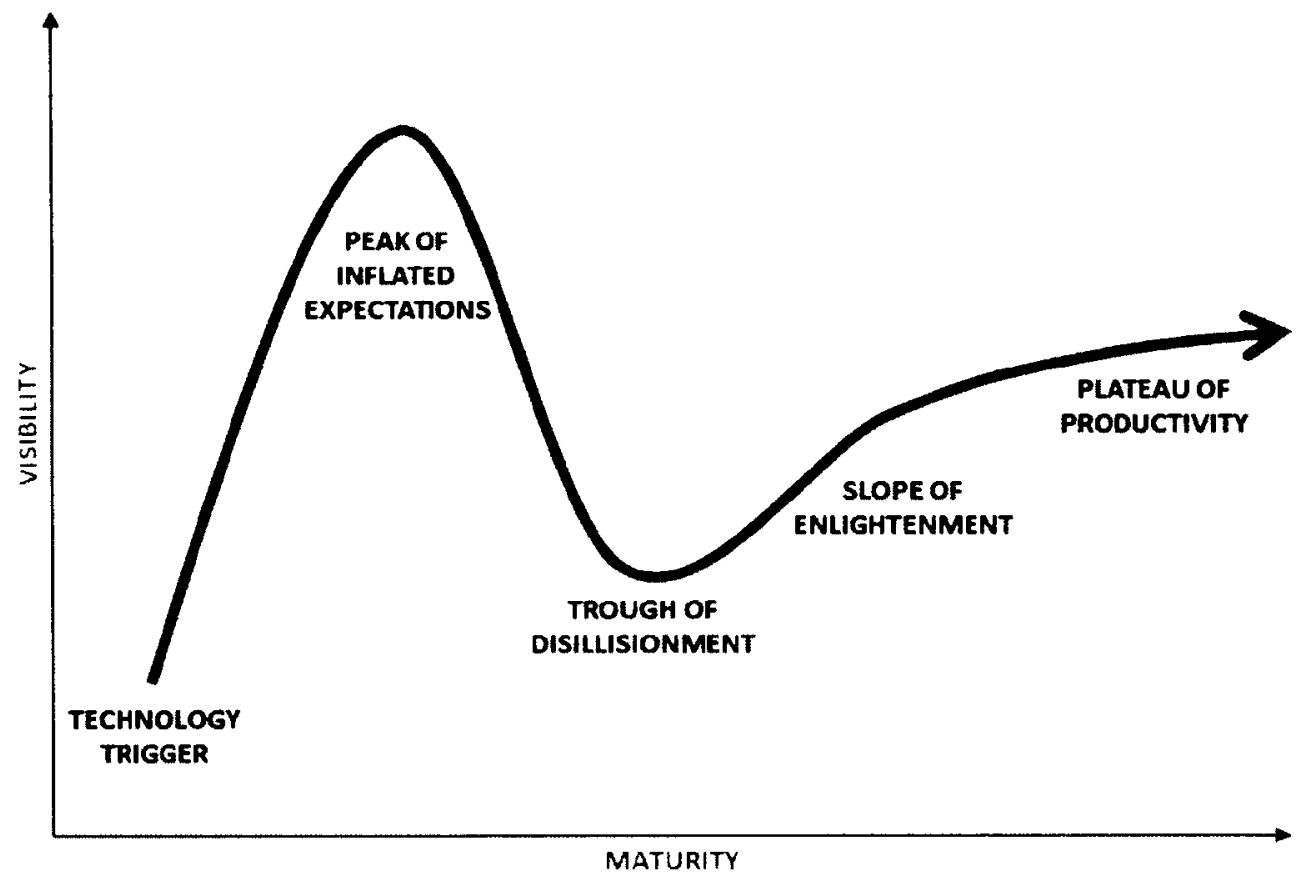

Source: http://www.gartner.com/technology/research/methodologies/hype-cycle.jsp

For the most part, the gamification movement has been epitomized by adding points and badges to systems. The implementation of points systems is instrumental in gamification processes as it indicates to users what actions will be rewarded, and suggests desired behaviours. For example, a points system with a leaderboard generally encourages competitive behaviour among users. Points systems thus express the select community values instilled by the site. For example, broadcasting the number of Twitter followers encourages the valuation of popularity and social influence, whereas awarding points for the number and quality of comments on other users' posts on sites such as The Escapist encourages the valuation of social interaction, as well as creates a filtering 
mechanism to highlight insightful, humorous, or useful comments.

Dedicated companies offer gamification services that layer points and levelling attributes onto existing third party websites (Bunchball, Big Door). Yet, as argued by game designer Margaret Robertson, these practices are really "pointsification":

What we're currently terming gamification is in fact the process of taking the thing that is least essential to games and representing it as the core of the experience. Points and badges have no closer a relationship to games than they do to websites and fitness apps and loyalty cards. They're great tools for communicating progress and acknowledging effort, but neither points nor badges in any way constitute a game. Games just use them - as primary school teachers, military hierarchies and coffee shops have for centuries - to help people visualize things they might otherwise lose track of. (2010)

Robertson argues that most proponents of gamification mistakenly equate pointsification with gamification. While points are an important motivator, the focus on points oversimplifies what makes games games. Inevitably, business that turn to pointsification will be disappointed when these efforts fail to imbue their product, service, website etc. with the power and allure of a compelling game such as Minecraft, Frontierville or WoW.

Much lauded gamification projects such as foursquare are already in decline as users become bored with simple badge systems (Evans 2010). These gamification efforts currently disappoint because they focus on points systems and ignore what really makes games compelling: the consequences of failure, and the elation of success. As Robertson argues, without failure, the challenges of gamification are meaningless:

A world of badges and points only offers upwards escalation, and without the pain of loss and failure, these mean far less. And when this upward escalation is based only on accumulation of points, rather than on expressions of my choices and my skills, then this further strips out the sense of agency and competence, so crucial to the emotional and neurological buzz we get from gaming. (Robertson 2010) 
Robertson emphasizes that unending 'upward' progression, without any threat of failure, becomes boring extremely quickly. Following these arguments, many gamification efforts promise fun and games, but are simply shallow veneers.

A related critique of gamification systems centres on the meaninglessness of rewards systems. Critics argue that system designers and implementers are the real beneficiaries of gamification, that gamification is a governance technology that offers little benefit for users. A reader comment on Jason Della Rocca's gamification article in The Escapist exemplifies this:

What I find amazing is that they've gotten enough people to believe that. The real reason they have achievement/trophies is to act as like a Nielsen rating for publishers. With the achievement trophies, they can peek in and see how many people played how far into their game, and how compelling the various elements were. They've just managed to convince gamers that it's a treat for them.

I don't know if you remember but games used to reward us before achievement/trophies. Actually they used to reward us better. For example in the previous generation GTA games if you completed the vigilante missions, you would receive an armour bonus. In GTA4, all you get is a crappy trophy. It's a matter of relevance. We used to get rewards that were relevant to the game we were playing. Now we get these arbitrary rewards that aren't relevant (on the contrary, they're pretty much worthless) to anything. (comment on Della Rocca 2010)

Marketers seize upon gamification because of the low cost of virtual rewards and the high rate of return in terms of collecting users' demographic data, as well as promoting increased engagement with the product. But, as demonstrated with the above quote, clearly, this technique will not be successful if the rewards carry no real utility or meaning. Despite current critiques of gamification, I believe, along with others (McGonigal 2011), that gamification will reach a plateau of productivity as techniques from games are taken up more and more in non-game applications. Unlike other 
commentators who focus on 'fun', however, I think the allure of gamification is founded in the surveillance and quantification of the self that current successful gamification practices deploy. The following case study of call centre workers exemplifies this.

\subsection{Case Study: Gamifying the Call Centre}

Hierarchies in the workplace are problematic because they are too tightly bound and constrained. They cannot keep pace with new technological innovations, let alone inspire them. In the Projective Cité, much like Bauman's liquid modernity, "empowered" free agents are more agile and innovative when they are free to do what they like (Bauman 2000). Most traditional corporations are too burdened down with bureaucratic rules, reporting, and accounting, in order to rapidly adapt to changing environments.

Gamification aims to provide employees and employers alike with the data analysis and data visualization tools needed to assess the situation and move independently towards goals. Moreover, gamification promises to constrain this independent movement within a rule-bound system, channelling behaviour towards productivity and efficiency, as well as fuelling it by drives for fun and self-mastery.

Julian Dibble, Nick Yee and Kline et al. have all argued that games are a form of socialization for high-tech work practices, such as fostering the ability to multi-task and the ability to parse multiple kinds of information being received simultaneously (Kline et al. 2003; Yee 2006b; Dibbell 2006). As put by Kline et al.:

[T] rhythms, speeds, and textures of the computerized environment; cultivating digital aptitudes; squeezing out of or devaluing other nonelectronic capabilities; [and] socializing players as subjects of and for a high-technology 
society... (2003:55)

The digital games of today do considerable work in shaping the office workers of tomorrow. But in this case, the digital games of today are the office of tomorrow, providing examples for CEOs about how to use technology and gamification to increase worker productivity, reduce absenteeism, and improve worker satisfaction.

In his work applying gamification to the workplace, Byron Reeves, along with J. Leighton Read, argues that victory conditions in employment are often nebulous employees do not know what to do in order to get a promotion. ${ }^{50}$ In contrast, the rules in games let players know what it takes to get from A to B, and accordingly set out what needs to be done for success. His company, Seriosity, attempts to create victory conditions for workers in terms of gamifying their work environments in order to increase collaboration and cooperation in decentralized networked environments and encourage innovation in hierarchical environments that are overwhelmingly bureaucratic, slow, rule bound, and risk averse. In this case, gamification serves as a neo-liberal Taylorist practice, providing moment by moment feedback on the minutia of employees' actions, subtly using rewards and incentives to increase workplace productivity while preserving the individual autonomy prized by the New Spirit of Capitalism.

After studying students playing World of Warcraft, Reeves and Read summarized some of key ingredients that make compelling MMO games and that could be exported to workplaces. Key elements of Reeves and Read's workplace initiatives are the real-time feedback to let the workers/players know moment by moment how well they are doing,

50 This is a particularly common problem in the Projective Cité. Networked models of production reward flexibility, adaptability and mobility rather than completing a predetermined set of tasks or increasing efficiency by a set percentage. Thus it is much more difficult to determine whether one is performing according to expectation. 
as well as more long-term feedback in relationship to larger goals, and the use of ranks and levels that work to motivate users and help them plan for future action. The use of avatars to represent the self and game narratives are also leveraged by Reeves and Read to promote worker engagement and increase workplace productivity. Workers undertake timed 'quests' that force them to work cooperatively in time, as well as hurrying to meet their goals.

Reeves and Read took these elements and used them to re-imagine call centre work, in the hope of improving employee retention, which averages between ten and eleven months per employee, and reducing absenteeism. They acquired the code from the Puzzle Pirates game and imagined how it could be changed to accommodate a call centre worker's daily tasks. ${ }^{51}$ In the scenario recounted in Total Engagement, employees signed into the game each morning and selected a team to work with. Assuming the role of pirates, each team took a ship and quested for treasure. The interface allowed the employee to click on their teammates' avatars to automatically check on their progress, (indicated by a range of metrics such as their level, how many calls they fielded that day, and, on average, how long they took on calls). This allowed the employees to check on and immediately offer aid to other workers who may have encountered difficulties. Teams received virtual treasures and points for completing each task and meeting overarching productivity goals.

The impetus for Reeves and Read's work was the realization that many jobs, including that of call centre workers, simply could not be automated as they rely on tacit

51 It is unclear from both Reeves and Read's writing and their public talks how far beyond conceptualization they took this project. Re-envisioning the call center is a prominent topic in Total Engagement, however, they do not publicize any concrete data from the project. This certainly raises issues about the gamification movement being founded on hype and PR rather than actual practice. 
knowledge. In order to increase productivity, workers needed to be motivated. Having fun, enjoying one's job, and deriving satisfaction from one's work are key to workplace motivation. Through observation, both of the workplace and of game spaces, as well as reviewing the literature on workplace management, Reeves and Read determined that giving these workers freedom to innovate (i.e. governing through freedom) was essential. In their own words, "you can't command the things you want from tacit knowledge workers; you have to create the conditions in which they want to give you innovation, collaboration, and insight" (2009:38). Productivity is inspired by setting the overarching narrative and goals, but giving workers freedom to choose how they achieve these goals.

In particular, Reeves and Read noted that most employees desire more purpose in their work; they want to have goals and clear feedback on their progress. They want to feel that they are working for something larger, and better than just themselves. Moreover, they feel hierarchical control and bureaucratic systems constrain any creativity and freedom they may have. Gamification is seen as the solution to these issues.

Gamification provides an overarching narrative, especially if the company narrative is too bland or uninspiring (for example, inspiring call centre workers to sell more vacuums). Game narratives and epic tales of conquest and teamwork can provide a sense of purpose. As in games, the rules and boundaries of the system are clearly laid out, but the employee is open to experiment, explore, and discover methods that are optimal for their purposes. Workers 'play' within the rule-bound system. Goals and feedback are clearly communicated. Ways to accomplish tasks are signposted along the way, but there is room for self-direction in terms of selecting tasks and timing. This exemplifies neoliberal 
methods of decentralized control. ${ }^{52}$

In the case of Reeves and Read's call centres, workers would be free to select from a variety of missions and quests. They would be free to do as they will, as long as what they do consists of fielding calls. In this way, gamification is different from traditional forms of Taylorization, which advocate a single, most efficient method of production for every task. Gamification is, in fact, Taylorism 2.0, providing workers the incentive to continually improve efficiency, to innovate and find creative methods to field the greatest number of calls. As call centre employees' skill improves, the challenges to which they would be subjected also increase. The bar for achievement is constantly raised, not allowing workers to become complacent and rest on their laurels. Most importantly, workers would be given a sense that they are free from the tightly constrained hierarchical control, and instead are 'playing' with work.

A large part of this play is rooted in surveillant practices. In Reeves and Read's scenario, because every action the call centre workers undertake is in within the virtual space created by the game, it is simple to monitor productivity. Work takes place within a walled garden, where every keystroke, conversation, and action is automatically surveilled (Whitson 2010b). While this level of surveillance is not new to call centre workers (Ericson, Doyle, and Barry 2003; Ball 2002), what is new is the way in which employees are actively enrolled in this surveillance project. Instead of resisting the monitoring of their every action, workers willingly submit to surveillance because they

52 One of the key problems of the Projective Cite is that increased worker autonomy equates to increased chances for an employee to move in a 'wrong' direction. While Boltanski and Chiapello posit that this can be addressed by internalizing a new business ethics and self-control that encourages workers to follow the company vision, gamification provides a different alternative - giving the impression of worker autonomy, while tightly constraining their actions to in-game options. By necessity, this sacrifices opportunities for worker innovation. 
are rewarded by it - being surveilled is the price of participating in the game, which replaces the dreary monotony of call centre work with a more appealing narrative of swashbuckling pirates and pillaging. Broadcasting to one's coworkers and supervisors to the second - how long one spends on calls, or how many calls one has fielded, is not an intrusion phrased in Orwellian terms. It is a way to earn victory points and to chart one's progress as one levels up their avatar and completes quests.

The systems built by Reeves and $\operatorname{Read}^{53}$ and other gamification practitioners are created to replace more hierarchical (and expensive) systems of online governance. Gamification practices, especially the awarding of points and special status for those that exhibit desired behaviours, allow for the shaping and regulation of behaviour through technological means that are seemingly objective. Doing away with human intervention and top-down governance, users are encouraged to govern themselves: "When workers are engaged by rewards that are intrinsic to their task rather than controlled by bosses, organizations can better decentralize" (Reeves and Read 2009:207).

This self-governance is predicated upon a hyper-transparency of information, and using this data to normalize one's behaviours. Metrics collection and analysis is automatic and presented in easily understandable visuals on a worker's game dashboard:

Players can easily see for themselves where they fit in and how well they are contributing and adjust their behaviour accordingly. This is a huge advantage and, we think, an important explanation for why lessons from these environments are the next frontier for enhancing leadership functions... The dashboards in games are like a streaming video of player' resumes. A huge bonus is that much of the information doesn't have to be provided by the person it's about (the biggest problem with any system that depends on

53 Beyond their call center scenario, their company offers a number of gamification services, including a game to monitor energy consumption, and Attent, a service that helps employees manage "information overload". 
human creation of metadata). Much of the information is automatically made transparent as the computer tracks play. (Reeves and Read 2009:169)

In true neoliberal fashion, supervision and hierarchical governance are less important because the gamified work environments contain so much information. This information not only lets each worker know exactly where they are in the game, it also identifies their place in the corporate hierarchy, making apparent competencies and talents as well as weak spots and failings.

This information is hyper-transparent because it is broadcast company-wide, enabling co-workers and supervisors to precisely measure one's merits with a quick glance at the game dashboard. Management directly benefits from releasing this data, as it encourages a "a looser hierarchy where people are expected to make their own decision or organize organically in groups that could process the information together" (Reeves and Read 2009:169). Employees monitor themselves, hastening their pace if they feel they are short of meeting their goals. Moreover, these systems allow for lateral surveillance, as clicking on the avatars of coworkers immediately indicates who is productive versus who is dragging the team down. While employees may not care about meeting their own productivity goals, they perform in order to avoid the censure of their co-workers. Friendly competition to be the best in the office may further incite productivity. As Reeves and Read describe, "this is orders of magnitude more and faster information than is typically captured in most real work. Players enter a different mindset about reputation: it's not only something that's developed and repaired over a career, it's something that's important (and could change) in the next minute" (2009:76). With minute-by-minute monitoring, gamifying the workplace results in a game that is never 
turned off.

Gamifying the workplace also results in a game that is never finished. Like the continual introduction of increasingly elite Amex cards and the expansion of top levels in WoW, it is relatively easy to add new goals, tasks, and missions. New mechanics, new narratives, and new levels can be added in order to maintain the attention of workers, while simultaneously maintaining the underlying scaffold of metrics, data visualization, and feedback. Ultimately the barrier for success for gamification projects is not high. ${ }^{54}$ Reeves and Reads' projects do not have to compete with WoW or FrontierVille, they simply need to show incremental improvement in the workplace, such as lower absenteeism, less churn, or slight increases to productivity and worker satisfaction.

\subsection{The Theoretical Implications of Gamification}

The rise of gamification as a technology of governance has important sociological implications. Gamification efforts emphasize and encourage values such as competition, advancement, efficiency and accumulation not only in game spaces, but in non-game spaces as well. Gamification fosters a technoliberal American Dream, telling users that if they work hard enough they can achieve victory - in games and in real life. Of course, hard work in this case is not represented by back-breaking labour, or even bootstrapping entrepreneurship. Rather, gamification rephrases the American Dream in terms of using technology to master and shape the body, in the process creating a victorious healthy

54 This was confirmed to me by a Seriosity employee. Their products do not have to compete with games, but rather create working conditions that are just slightly more preferable than what went before. Worker resistance to these gamified scenarios, especially in the case of call centres which are already highly surveilled and governed, is thus assumed to be lower than resistance to the forms of work that preceded gamification. 
machine, one that has many friends and followers, and is a productive worker and savvy consumer. The surveillant practices that are embedded in technology become essential tools for measuring one's progress, providing feedback, and highlighting routes toward this success, whether it is running for 30 days in a row, losing 15 pounds, earning a promotion, or getting more miles to the gallon while driving. Under the Projective Cité, citizens are governed through a web of visibilities, wherein practices such as gamification open our private intimate spaces to visibility, thus "locking each 'free' individual into a play of normative gazes" (N. Rose 1999:73).

\subsubsection{Feedback Mechanisms in Games}

Data visualization techniques are the key element that gamification takes from games, and are an essential tool in shaping users' behaviour. As evidenced by Reed and Reeves:

Game interfaces set a new bar for feedback. At any one time, Helen sees progress bars, zooming numbers, and status gauges, all in a well-organized dashboard that lets players know how things are going, good or bad. Numbers indicate the health of players, the time left before an attack, the amount of gold accumulated so far ...(2009:71)

Games excel at providing precise real-time feedback to help players chart their current progress and determine how to advance. Feedback thus channels behaviour; steps towards a goal are encouraged in multiple ways and channels, while steps in the 'wrong' direction are penalized. Awarding virtual goods and points in order to reward 'correct' behaviours is ubiquitous, but feedback also takes other forms, for example, aural feedback in terms of words of encouragement and changes in sound effects and music, visual feedback, in terms of charts and statistics of progress and visual effects, and even 
physical feedback in terms of rumble-packs in controllers that vibrate in response to a player's actions.

In games, feedback is negative as well as positive. Failure in games, such as a character dying and having to restart a level, is common (as of yet, most gamification efforts have not perfected this negative feedback). But this failure is welcome in comparison to the real world. There are relatively few consequences for failing in games (Juul 2010) and the negative feedback provided contains information that helps the player learn and thus achieve their desired goals. For example, in WoW, a raiding party may fail many times before finally defeating a monster. Each failure provides valuable statistics on what techniques are successful (i.e. attacks that inflict maximum damage, team formations that provide an optimum balance between tanks and healers, etc.), and what actions to avoid. Each consecutive failure comes with an incremental improvement in strategy until finally, the raiding party is victorious. Failure is part of the game. It provides valuable information on how to become better.

Feedback in games is both short term and long term. Because data is constantly gathered in games, feedback can be immediate, for example, providing a WoW player with real-time per second data on how they are faring in an attack. But feedback also takes mid- and long-range forms, providing information on how a player is progressing with goals that take weeks, months, or even years to accomplish. For the most part, feedback in the real world is much more infrequent and difficult to accomplish, largely because the automated cycle of data collection, compilation, analysis, and feedback is simply not established. As argued by Reeves and Read (2009), in the workplace feedback 
is infrequent: most workers only receive input about how they are doing at their annual performance reviews. Porting the feedback methods used in games to non-game activities thus makes sense:

Quick feedback creates immediacy and contingency in the interactions. When you make a new move, you know quickly whether the action was right or wrong. The close connection between behaviour and feedback (it's usually obvious which reinforcement applies to which behaviours) increases the likelihood that the reinforcement will be effective. (Reeves and Read 2009:72)

The importance of feedback in gamification efforts is the quantification of the self, and the trend towards measuring areas of our life that were previously private and unquantifiable. From advertising how many friends we have on Facebook to chronicling our weight loss history using Wii Fit, our personal lives are increasingly data driven.

\subsubsection{The Role of Surveillance}

The key commonality between the myriad examples of gamification is the leveraging of surveillance. Whether online or off, users' behaviours are tracked via technology that monitors progress. These metrics are then used to provide incremental feedback to the user, thus indicating what they need to do next in order to achieve their goals. Accordingly, the promise of a 'game' and the desire to level up and win is used to inculcate desirable skill sets and behaviours. Gamification is thus rooted in surveillance; providing real-time feedback about users' actions by amassing large quantities of data and then simplifying this data into modes that easily understandable, such as progress bars, graphs and charts.

From a surveillance studies perspective, what is really interesting is how 
gamification practices turn traditional surveillance tropes on their head. Surveillance is not phrased in terms of dystopic panopticons that have been over-emphasized by most Foucauldian scholars (Valverde 2008), nor is it couched in terms of societies of control, access and exclusion (Deleuze 1992). Surveillance is phrased in terms of enabling free play and promoting engagement, accordingly there is less reason for users to 'opt out' or resist gamification, even if it is imposed upon them by others such as employers. But this free play does not mean that workers are unsupervised. Workers are surveilled more closely than ever before. Everything they do online is logged and databased. Performance metrics become indicators to the inner self. They are "a complete and public package of competency, prowess, and experience...that makes it more true in the game than in real life that what you see is what you get" (Reeves and Read 2009:75). What is new is that surveillance is framed as fun (Albrechtslund and Dubbeld 2005).

To reiterate the central tenets of governance via gamification: Supervision is not about discipline and control, but is geared towards providing meaningful feedback and rewards. Workers (or consumers) are rewarded whenever they make incremental progress towards larger goals. In order to be rewarded, it is necessary to use surveillance in order to measure and quantify one's progress. Performance metrics and feedback are overwhelmingly positive, and focused on improvement, reward, and engagement rather than highlighting deficiencies. Of course, failure still exists, but the risks and punishments for attempting something and then failing are not as severe. Negative feedback works to highlight areas that require improvement and suggest changes in tactics that may help in achieving success, rather than punishment. Thus, users can 
clearly decipher what they need to do in order to progress.

This form of surveillance would have been impossible to carry out in the past. The sheer amount of data collection, analysis, quantification, and feedback, especially on a moment-to-moment basis would have been entirely impossible. But in automated closed systems such as gamified workplaces, or even the walled gardens of games and social networking sites, the collection and analysis of data is much easier. Progress is easy to chart and reward, especially as virtual rewards and reputation scores do not have to cost anything. The emphasis is on fun, at least in terms of the marketing of gamification, if not the actual application. Whereas notions of surveillance often fixate on punishing people who have done wrong, or promoting normalization, surveillance is fun. It is gamic. It is about noticing and rewarding people who are doing well, and perhaps offering help. direction, tips, and motivation for those who are not. In this way, following the advice of Mariana Valverde, perhaps we can move beyond the panopticon and Orwell and look at how surveillance, at least as envisioned by Foucault's original works, can operate in much more varied and complex ways (2008). In terms of resistance, techniques to neutralize surveillance such as refusing to participate, distorting one's data, or breaking the system (Marx 2003) are ineffective, as they also entail a rejection of the accepted benefits (e.g. accurate feedback that aids in self-improvement)..$^{55}$

55 Gamification services are generally opt-in and purchased by the user. Accordingly, there is little reason to falsify data or otherwise resist this surveillance. I have written elsewhere on the allure of playing with and exploiting game systems (2010a). However, more studies on gamification in practice - especially mandatory gamified workplaces and classrooms - would have to be carried out to accurately comment on the forms of resistance in gamified domains. 


\subsubsection{The Quantification of the Self}

A key concept in the use surveillance to govern through freedom is the Quantification of the Self. We are used to measuring and quantifying many things in our lives - from optimizing assembly line production, to measuring how fast our computers operate, to grading our intelligence, to using software to clock how many hours and minutes each day we work. Yet, there was always a separation between personal lives and public. Our performance at work and school was measured but we left this quantification behind when we got home. With the aid of technology, gamification now enables us to measure and chart what was previously private. It is now relatively simple to quantify and analyze patterns in our sleep, exercise, sex life, food intake, mood, location, alertness, productivity and even our spiritual well being. We effortlessly track and measure, display and share all of this heretofore unknown data using our computers, smart phones, and gaming consoles.

While the quantification of the self has commonalities with the time-honoured tradition of journaling and the care of the self detailed by Foucault (1988), and the listmaking more recently described by Umberto Eco (2010), what is different is the precision, complexity and the amount of the data collected, as well as the way it is ultimately presented back to the chronicler. Instead of leaving it up to us to decide what is worth chronicling, and then delegating our spotty memories to provide the details, the journaling process in the era of gamification is automated, enabling incredibly precise details. For example, instead of noting in my diary that I ran today, Nike + tells me that I ran 4.2 kilometres, in twenty eight minutes and fourteen seconds, 42 seconds slower than 
my personal best. I can tag the run with details about the weather and how I felt, and later look at the accumulated data to determine that I run best when I am sad and the temperature is $15^{\circ} \mathrm{C}$. Nike+ records the route I took through the city, how many calories I burned, the music I listened to, and how many days within the last months I missed. It charts the incremental improvement in my running times over a period of months. Moreover, it can broadcast this data to sites like Facebook and my Nike profile page, allowing me to share this detailed information with friends, initiate running challenges, or even follow a friend's running route through Ottawa.

Processes of gamification leverage surveillance in a way that promotes a care of the self, blurring qualitative assessments of well-being (e.g. by measuring emotions, moods, level of arousal) with quantitative means (e.g. the statistics and graphs the come from the data collection). Projects of gamification and the quantification of the self are focused on surveillance because the automatic collection and analysis of metrics and other data enable clear, easily parsed forms of feedback that can be presented in an interesting visual manner (charts and percentages) that we are already familiar with from our workplaces and schools.

The 'game' involved in gamification projects such as Nike+ is in setting challenges and goals, both short term and long term. ${ }^{56}$ Charts, graphs, and statistics are

56 For example, with Nike+, each run comes with its own goals (such as setting a personal best), and spoken feedback motivates me through the run, as well as music that suits my pace (as well as a selected 'power song' to help increase my tempo). As my total running distance accumulates, I earn rewards such as congratulation messages that are pre-recorded by celebrity athletes, virtual trophies, and certificates of achievements that I can print out and hang on my wall. I can "level up", which is visualized in terms of earning colors, moving from Yellow (0-49 $\mathrm{km}$ ) to Orange (50-249 $\mathrm{km}$ ) once I break the $50 \mathrm{~km}$ mark, going through the rainbow as the total number of kilometres I have run accumulates. If I decide to train for a marathon, there are training coaches that I can download, planning my workout regime for weeks in advance, allowing me to ramp up. 
automatically compiled, transforming what is essentially a large database of meaningless numbers into something that users can quickly parse and understand. By gamifying everyday tasks such as exercise and healthy living, users make solitary and tedious activities somewhat more enjoyable. Failures to make gamification and self-mastery 'fun' do not equate to a failure of gamification. Users enrol in gamification programs out of curiosity, and continue with them because the data provided is so compelling: "they continue because they believe their numbers hold secrets that they can't afford to ignore, including answers to questions they have not yet thought to ask" (Wolf 2010). The quantification of the self - by compiling the intricate details of our lives and then rebroadcasting them to us in new ways - promises to tell us something about ourselves that we did not already know.

The quantification of the self is not new, but gamification greatly expands its scale and scope, as well as its effectiveness at telling us our 'secrets'. As eloquently stated by Gary Wolf, we track ourselves all the time:

We step on a scale and record our weight. We balance a checkbook. We count calories. But when the familiar pen-and-paper methods of self-analysis are enhanced by sensors that monitor our behaviour automatically, the process of self-tracking becomes both more alluring and more meaningful. Automated sensors do more than give us facts; they also remind us that our ordinary behaviour contains obscure quantitative signals that can be used to inform our behaviour, once we learn to read them. (2010)

The quantification of the self allows us to replace the holes in our memories and the vagaries of our intuition with something more reliable and something more objective. There is naturally some distrust associated with this continual monitoring and the coldhearted calculations that distil the complexity of our lives into colourful stream graphs, 
tag clouds and infographics, but we "tolerate the pathologies of quantification - a dry, abstract, mechanical type of knowledge - because the results are so powerful. Numbering things allows tests, comparisons, experiments. Numbers make problems less resonant emotionally but more tractable intellectually" (Wolf 2010). Moreover, we tolerate the quantification of the self because it is so often phrased in terms of playing a game that will make our selves better; whether it is improving our bodies, our minds, or even our social lives. In this sense, gamification is a tool that goes far beyond disciplinary normalization. With normalization, individuals are "individuated by comparing their characteristics - height, weight, circumference of skill, and later intelligence and moral worth - with those of the population as a whole" (N. Rose 1999:75). In contrast, with gamification, we strive not to be normal, but to be the best, to be supernormal. ${ }^{57}$ With gamification, quantification rapidly proliferates and becomes a key measure of selfworth. Self-improvement does not end once one attains the average, but rather is a continuous levelling-up process.

\subsubsection{The Implications of Objective Quantification}

The continuous levelling-up and measuring self-worth via quantification has definite benefits as well as drawbacks, the latter of which have particular resonance for later chapters of this thesis. Gamification practices build upon psychological needs such as self-mastery and self-improvement, reputation and status building, achievement and reward (see A. J. Kim 2000, 2009, 2010). These efforts foster a sense of autonomy and

57 An example of the compulsion to become supernormal can be found in the ubiquitous leaderboards that encourage users to become the best player. 
self-efficacy as the player selects what quests to complete, how to do so, and on what timeline. Jason Della Rocca sees gamification as providing more benefits, in that the scores and achievement levels provide an "objective" form of self-validation. More importantly: "With regards to social validation, games provide an unbiased judge: The rules of the system are arbitrated by the unfeeling computer. This is transparent, highly detailed and visible to the community as a whole" (Della Rocca 2010). Computers now collect, keep track of, and display data about users in the form of metrics and user statistics. The computer is now the assessor of reputation, with the assumption that technology is more objective and infallible that humans. As elaborated by Reeves and Read in their work on gamification of workplaces, "Players (and leaders) learn to trust game metrics, in part because it's difficult to cheat. It's important for gamers to know that the data are objective and less susceptible to social influence than might be the case in settings with fewer environmental confirmations of performance" (2009:169). Ultimately, we believe that computers are better at faithfully evaluating a person's worth. The data does not lie.

While the user believes that this validation is unbiased, it is important to emphasize that particular values are deeply inscribed in the system. The system rewards the user for their actions and points are generally rewarded in a transparent manner, thus seeming objective and fair. Yet the valuation of what actions earn points is set by the designers of the system, thus system designers have more control than ever (Whitson 2010a). They can reward subtle changes in behaviour to inspire and evoke optimum performance. Only certain behaviours are worthy of notice and rewards. So, in the case of 
rewards in systems like foursquare, brand loyalty, return visits, and consumption are all worthy of rewards. In the case of a call centre, answering as many calls as possible within a time limit is valued.

While the dashboards of these systems are seen as transparent - showing users exactly what other users are doing - the inner workings of the system are opaque (Whitson 2010a). Moreover, they can be tweaked and altered as the game progresses. The true workings of the system are hidden. Every aspect can be monitored and controlled. Questioning what behaviours are rewarded and what behaviours are ignored is not done, because the rules of the game are hidden with the black box of technology.

Statistics, measurement practices, and classification schemes more generally are never just a benign assessment of the world, but change our conception of the world and our understanding of ourselves (Huff and Geis 1954; Bowker and Star 1999). Quantification practices tell us what is important to measure, how we should measure it, and indicate how we should change it. On a technological level, it is much easier to measure and reward some behaviours in comparison to others. As an example, this is why killing in games is such a popular activity - it is perfectly suited to the computational aspects of a game. As stated by game writer and designer Darby McDevitt, "Death is a Boolean operation: something is either alive or not-alive, which makes determining victory conditions easy. Are you dead? Then you have lost. Is your opponent dead? Then you are winning; keep it up" (2010). In games, the conditions of success are clear and decisive. Common activities such as jumping, racing, shooting, and solving puzzles can all be scored or measured with a high degree of certainty. 
Game designers and game scholars alike lament games' current inability to adequately address areas of life that are not easily quantified such as affect (Kline et al. 2003; McDevitt 2010; Juul 2005). McDevitt gives an example of this difficulty:

If a designer wanted to make a game called Terminal Relations, say, in which the only goal was to comfort your pious, cancer-stricken grandfather in his final days, she'd probably have a difficult time designing the actual challenges. Matters of emotion, morality, empathy, religion, cultural identity, and the like, are difficult to translate into iterative mechanics because they are primarily psychological or interior phenomena with no clear victory conditions. (McDevitt 2010)

Not everything in life can be objectively measured, quantified, and turned into a game mechanic with clear victory conditions, yet this quantification and measurement of a person's worth is exactly what attracts proponents of gamification.

\subsection{Conclusion}

In this chapter, we saw how gamification practices, while operating under the umbrella of play, foster a quantification of the self: collecting, collating and analyzing minute data and providing feedback on how to better care for one's self. This quantification of the self feeds into neoliberal governance projects that promise to make daily practices more fulfilling and fun. Enabled by increased levels of surveillance (self-monitoring and otherwise), these projects use incentivization and fun rather than the traditionally ubiquitous motivators of risk and fear to shape desired behaviours. Becoming a victorious neoliberal subject is a never-ending process, guided by a teleology of constant and continual improvement, driven by an unending stream of positive feedback and virtual rewards, and fuelled by the notion that this process is playful and fun. 
The example of gamification presents an paradigm version of how idealized subjects of the New Spirit of Capitalism are governed through freedom. The citizens of the Projective Cité are focused on self-improvement, wherein their autonomous actions are guided by the objective numbers and feedback mechanisms of game-like applications. But how did games move from the sandbox to the office space? If traditional notions of games and play are focused on actions that are set apart from the real-world - actions that are inconsequential and frivolous - what ideological shifts must take place for games to be accepted as tools for productivity and governance? The next chapter addresses these questions, showing how the instrumentalization of play creates a governable space where before there was none. 


\section{Chapter 4. The Instrumentalization of Play}

Rationalizing processes have consequences for the way we think about and utilize games today. Different rationalities justify what games are allowed to do, and for what purposes games and play are suitable. This thesis argues that shifts in rationalities are linked to changes in underlying Cités, and consequently, what games do and how they are made are changing along with the New Spirit of Capitalism. In the last chapter we saw how gamification becomes a technology of governance, aligning with the networked logics of the Projective Cité which justify and provide value structures for the New Spirit of Capitalism. This chapter is about the ideological preconditions required for the gamification movement to take root, ideologies based on instrumental rationalities ${ }^{58}$ that have broad impacts on games and play more generally. It focuses on play itself, while the remaining chapters focus on the developers who create play spaces.

As games become institutionalized ${ }^{59}$ on larger and larger scales (such as FarmVille with more than 80 million players at its peak) they become systems of social rationality. Games organize players in a manner similar to how markets organize consumers and state bureaucracies organize citizens. Rather than being mere play, they articulate cultural and political meanings. As this chapter argues, these meanings

58 To avoid the confusion between "rationalities" in the Foucauldian sense, and "rationality" in the Weberian sense, in this rest of this chapter I use "Cités" to denote the former meaning and refer to "rationality" only in the latter sense. Superficially, it would seem that instrumental rationality would align closer to the Second Spirit of Capitalism and the Industrial Cite rather than the New Spirit, however I argue that instrumental rationalities remain, but are now couched in language of the Projective Cité.

59 By institutionalized, I mean that games become sites of large-scale organization, wherein formal and informal schemas, rules, norms, and routines become established as authoritative guidelines for social behaviour. The institutionalization of digital games is technologically mediated, leaving less room for players to spontaneously negotiate the rules and their exceptions. As Grimes and Feenberg argue, (2009) players' actions are increasingly reduced to a predetermined set of possibilities. Games thus produce of a form of institutional order, governing the behaviour of individuals within the player community. 
overwhelmingly favour rationalization, productivity, and efficiency. Ultimately, in the New Spirit of Capitalism, there is no room for unproductive play. Instead of the separation between work and play underlined by the Industrial Cité, in the Projective Cité, the lines between work and non-work are blurred - especially when non-work activities feed into self-development and thus increase one's employability in new projects. Accordingly, in this chapter I trace the changing notions of play, highlighting the shift in thinking that play is not inconsequential - that play can be work - as well as resistance to this idea.

My own normative stance on instrumental play has developed from the perspective of a player. Instrumental play results in games wherein players are focused on overcoming predefined constraints in a largely choreographed manner. This contrasts with games that are less goal-oriented and thus open up more heterogeneous range of actions. Instrumental play is not inherently negative - I actually prefer linear, singleplayer shooters premised on instrumental levelling up and goal directed behaviour. But, instrumental play is commonly coupled with the shift in thinking that play can - and should - be productive. Most of the time, players like myself are aware of and complicit in this production (e.g. user-generated content), even when what is being 'produced' is the responsibilized neoliberal subject (e.g. using games to lose weight). However, in many cases, the forms of production are less transparent or completely hidden from players. For example, when player data is collected, analyzed and used for in-game targeted marketing, or when achieving the goals of the game is surreptitiously linked to spending more money in-game (a common trait in free-to-play games on social networks), or when 
the game is explicitly developed to produce docile workers and thus mask the realities of the workplace. In these cases, I resent the lack of transparency and the extent to which the pleasures of play are commodified and/or leveraged to support less laudable goals. In this sense, I value play as a goal in and of itself, and thus dispute the arguments that play and games would be better if they were made more productive. ${ }^{60}$

\subsection{Instrumental Play}

The theoretical underpinnings of this thesis centre on instrumental play and the increasing rationalization of games. On the player side, our most playful and frivolous actions - the sheer joy, fun, and challenge involved in playing games - are leveraged to enrol us, as players, into propagating a social order based on notions of rationality, efficiency, commodification and surveillance. Changing ideas of what games can do (e.g. gamification) allows games to becomes greater spaces of governance. This chapter largely takes a player-centric approach to explore how the way we play is changing, while the remaining chapters take a more developer-centric approach in analyzing how this rationalization transforms game development from an aesthetically charged and creative set of practices into an increasingly passionless and professionalized instrumental process.

My analysis draws from four notions of instrumental play: as it was first

60 While it could be argued that many game scholars and developers romanticize play (for example, Miguel Sicart's emphasis on paidia over ludic games), I do not believe that this is necessarily a bad thing. To return to Mark Twain's definition of play from Chapter One: work consists of anything a body is obliged to do, and play consists of whatever a body is not obliged to do. The escalating efforts to make play more productive, serve non-game purposes, or even, to make player's behaviours more predictable - to me - endanger this fundamental aspect of play by binding it within layers of obligation and ulterior motives. Rather than being something intrinsically valued, it collapses under the weight of these other additional expectations. 
introduced by T.L. Taylor (2003, 2006b); and then later reworked by Miguel Sicart (2010); and, the instrumentalization and rationalization of play (aka the "ludification theory") proposed by Sara Grimes and Andrew Feenberg (2009). (The instrumental work/play of game development itself is described by Casey O'Donnell (2008) later in the thesis). I use these theories as a toolkit, pulling from each to refine my understanding of how play is changing alongside the massive economic shifts that are currently restructuring the game industry. ${ }^{61}$ In the following sections I will introduce and engage with each theory individually. But first, I will briefly define what I mean by "instrumentalization".

The instrumentalization of play is rooted in Max Weber's work on rationalization (2002). Rationalization refers to the process in which rational motivators for behaviour replace emotional, traditional, or value-laden motivations. David Garland, drawing from Jürgen Habermas, Theodor Adorno, and Max Horkheimer, defines rationalized social practices as those "rule-governed forms of social action which are calculated and calculable, based upon a self-reflexive knowledge of their aims and conditions, and oriented to achieving these ends by the most instrumentally appropriate means" (1991:179). These practices are contrasted with affective, customary, or traditional forms of social action. The latter of which are non-rational or quasi-rational because they are dictated by emotion, habit or other irrational factors. Rationalization is a modernizing development wherein social practices become better informed, more efficient, and more self-consciously adapted towards specific objectives. This 'progress' is attributable to the

61 Even more broadly, rationalization in the game industry parallels much larger trends in the way innovation and creativity in new media industries are harnessed by businesses espousing Projective Cité values. 
role of science, wherein technical knowledge - including objective data and mathematical calculations - replaces belief and sentiment as the leading determinants of action. In this context, instrumental reason involves calculation and efficiency, thus reducing all relationships to those of means and ends. Accordingly, rationalization has dehumanizing tendencies. While social practices and institutions are made more instrumentally effective and efficient, they become less compelling or meaningful because the emotional, affective, and spiritual components have been stripped away, thus fuelling a "disenchantment" with the world. As we will see in the following chapters, this rationalization has implications for how games are made (i.e. focusing on furthering efficiency, calculability, predictability and control in game development), but it also has implications for what games are.

I am not arguing that instrumental play is a new phenomenon, but rather that it is extending its reach (i.e. more play within games is instrumental) and its domain (i.e. instrumental play is leveraged in traditionally non-game spaces). Play has long been understood in relation to work, as a form of training for the capitalist workplace and a tool for inculcating social order. Most recently, Mary Flanagan has followed in this tradition, for example, using the context of Victorian dollhouses to detail how "playing house" normalizes and institutionalizes cultural norms of the time, especially in terms of feminine domesticity. She provides another example in the form of nineteenth century boardgames, which filled the expanding leisure time of the middle class with games that developed the thinking skills and the moral instruction deemed necessary for success in the industrial age (Flanagan 2009). Of course, capitalist industrialists have long realized 
that a workforce that played after work would be more productive. Leisure time fuels an industrious cycle: workplace productivity provides employees with capital that is then expended through the consumption of leisure activities. In order to continue these leisurely activities, employees return to productive labour, and thus perpetuate the capitalist cycle of work and play. ${ }^{62}$

However, as Sicart, Taylor, and Silverman and Simon all argue, this clear distinction between work and play is collapsing. As Silverman and Simon recount, "good work is play", and the values associated with play such as freedom, autonomy, and joy have become values associated with idealized forms of work (2009:354), especially in the new knowledge industry (O'Donnell 2008) and in the Projective Cité. At the same time, all of the above noted authors trace how, conversely, the values associated with work such as a focus on efficiency and organizational skills, a drive to accumulate and 'level up', and a dedication to grinding through long swathes of tedious work, have become associated with play. Increasingly, play has become productive, in terms of the creation of virtual goods with real world value. New terms have been created to describe this shift and the intertwining of consumption and production within games: "free labour" (Terranova 2000) “productive players” (Humphreys 2005) and "playbour” (Kücklich 2005). Clearly something is at work here: the rise of instrumental play.

In its general usage, the term "instrumental play" describes play that is focused on advancement within the game. Play is a means to an end (whether it be completing the game, beating opponents, etc.) rather than an end in itself. It is goal directed and centred

62 For an elegant review of the intermingling of work and play within critical social theory see (Grimes and Feenberg 2009). 
on rewards. While the rewards may be intrinsic to the game, such as earning points, they may also be extrinsic, such as gaining social status through the accumulation of $\mathrm{Xbox}$ Live achievements and trophies. Certain forms of digital games are instrumental by definition, in that they are developed to facilitate something other than just play. For example, all serious games are instrumental in that play is a means to impart an educational message and, ideally, change players' behaviours outside of games. This also applies to gamification, wherein both playful attitudes and game interfaces are leveraged to promote a wide range of behaviours such as weight loss, worker productivity, and fiscal awareness.

The instrumentalization of play can be described in general Frankfurt School terms $^{63}$ in that mainstream games and gamification movements are largely standardized, formulaic goods that manipulate society into passivity (although this process is far more complex than a simple hypodermic needle effect, where the intended message is directly received and wholly accepted by the player). The pleasures of play can be leveraged to create a docile and content population, and hide the fact that the instrumentalized games we play do little to fulfil our psychological needs. Rather than offering actual freedom and creativity, they offer the simulation of freedom and creativity.

As Casey O'Donnell cautions, we need to distinguish instrumental play from the foundational concept of instrumental rationality detailed above. The key difference here is that instrumental play "has no claim to the irreducible or absolute" (O'Donnell 2008:67). In fact, a key component of playfulness is the drive to always push up against

63 Sicart's argument in particular, seems premised on a divide between high art (paidic games) and low (ludic linear games). Parallels can be drawn between other culture industries here (i.e. cinema, literature, etc.); however, in games the emphasis is on interactive ideals (freedom, play and creativity) as well as the intellectual and aesthetic elements that traditionally characterize high culture. 
what is considered irreducible (see Whitson 2010a). Similar to the methods of scientific enquiry espoused by Enlightenment thinkers, instrumental play is about discovering associations, analogies, and relationships, but unlike instrumental reason, instrumental play makes no claim about an absolute character of those suppositions. There is always the assumption that what players are playing with (or in the case of game development itself, working with), might swerve and send the player in new directions. ${ }^{64}$

\subsection{Miguel Sicart and the Rules of the Game}

For Miguel Sicart, the rise of instrumental play is defined by the current trend to favour "'gameness" over playfulness in games, at least to a certain extent, or how digital games are about achievements and victory more than about play itself" (interview with Bateman 2010). Increasingly, Sicart argues, games are about goals, rather than the pure pleasure and performance of play. Sicart highlights the role that game studies academics and designers have in promoting instrumental play, and argues that defining, designing, and analyzing games solely as rule-based systems is to blame. For Sicart, academics and game designers define and speak about games in instrumental terms. This, in turn, influences others to speak about and design games according to instrumental terms. Players then adopt these instrumental terms and values, leaving no room for other forms of play. To understand the implications of Sicart's view, we need to turn to the recent history of game studies.

In "Against Procedurality" Sicart traces the history of proceduralist discourse in

64 I would argue, however, that the space between instrumental play and instrumental rationality diminishes with the increased reliance on using metrics and big data to predict player's behaviour and, in near real-time, preempt responses deemed by game publishers to be unfavorable to their interests. 
game studies (Sicart 2011). Continuing the work started by ludologists, proceduralists argue that by their very nature, digital games are different from other media such as books and board games and thus deserve to be "analyzed through the ontological particularities that makes computer games unique" (Sicart 2011). Characterized by the work of Ian Bogost (2006) and rooted in ideas of Janet Murray (1997), proceduralists prioritize the understanding of games as rule-based systems. The central premise of proceduralism is that games are defined by their rules, and through these rules games present embedded values that are appropriated by the player. Thus the rules, the values embedded within the game, and the appropriation of these values as the player plays through the game become key sites of analysis (Bogost 2007).

While the idea that objects can embody values in their design is not new (Winner 1986; Latour 2005), Sicart suggests that the proceduralism, as a mode of theorizing games, is technologically determinist: defining games and their meaning by their rules alone and ignoring the importance of both play and players. He argues that technological determinism is found in the analysis of games (Flanagan 2009; Juul 2005; Bogost 2006, 2007), but, more importantly, it contains an implicit approach on how to design games that is becoming much more common (Salen and Zimmerman 2004; Brathwaite 2010), meaning that more and more games are designed to be procedural. Following this approach, designers "provide players with guided experiences in which the very act of play is controlled and oriented" towards the completion of predetermined goals (Sicart 2011).

Sicart suggests the problem with proceduralism, especially in the design of games, 
is that the authorial intent and the creation of rule sets takes predominance over players actually playing the game. Games, or more accurately, the rules of games become a text that is read and assimilated by the players. Accordingly it is the text, and not the play that is emphasized:

Game designers are supposed to create play, that is, a particular behaviour in players. Proceduralists believe that those behaviours can be predicted, even contained, by the rules, and therefore the meaning of the game, and of play, evolves from the way the game has been created and not how it is played; not to mention when and where it is played, and by whom. (Sicart 2011)

Players are important only as activators of the game. Thus, the understanding of games and play is equated to understanding rules. This downplays player creativity and expression and instead prioritizes a rational progression through the game, following a path that has been selected beforehand by designers. The efficient player - the one that learns the system and selects an optimal route towards the predefined goal - is the successful player.

But Sicart reminds us that: "Games structure play, facilitate it by means of rules. This is not to say that rules determine play: they focus it, they frame it, but they are still subject to the very act of play" (2011). While rules are a part of play, play cannot be summarized by the rules alone, play is less about the rules of a game and more about the player engaged in the game. The very nature of play encourages testing, bending, and even breaking the rules. Playing a game necessitates learning the rules and testing their boundaries (e.g. how high can I jump? Who/what can I shoot? etc.), while winning requires mastery of the rules (Koster 2005), and in some case bending (e.g. exploits) or breaking them (e.g. mods or cheats) in order to win. Players consciously decide to play 
with the rules and structure of the game (Sotamaa 2009:82). Mastering, bending, and even subverting rules is a part of play. For example, Salen and Zimmerman define play as "free movement within a more rigid structure" (2004:304). Using the example of the 'play' of a car's steering wheel, Salen and Zimmerman argue that play is made possible by rules and rigid structures. In other words, the play of the wheel is only made possible by the rigid structures of the steering column and axles, while game play is only made possible by the rigid structures of the rules. Because by nature a game has room for the movement of play, it is "always possible for players to drive a wedge into the system, bending it and transforming it into a new shape" (Salen and Zimmerman 2004:565). Play cannot and should not be reduced to rules. Play is the dialogue between the player and the system of rules. In this dialogue, the player may submit to the rules, but more often they push against, creatively appropriate and reshape these rules.

Yet the proceduralist turn has emphasized, both to academics and designers, that the meaning of a game is to be found in its rules. Despite the "mangle of play" (Steinkuehler 2006) and the inherent freedom emphasized by commonly held notions of play, Sicart argues that the predominance of proceduralist thinking by academics and designers leads to play itself being instrumentalized and seen as the mechanical outcome of precisely designed processes and rules. Designers provide players with guided experiences that control, channel and orient the very act of play: "Proceduralism, with its call for systems at the core of the essence of games and its disregard for expressive or ineffective play, turns the act of playing a game into a labor-like action, into work towards an externally decided, predetermined, and rational outcome designed by others 
than the players" (Sicart 2011). This results in instrumental play, which is further defined by Sicart as "the process of playing for other means, as play subordinated to the goals and rules and systems of the game. In this sense, play is subordinate to reason, to the logical of achievement and progression externally determined by the [designer]" (Sicart 2011). Ultimately, Sicart concludes that instrumental play reduces the player to a cog in a cybernetic loop and is becoming increasingly common not only in commercialized AAA games, but across the board, including idealistic indie games and well-intentioned serious games.

\subsection{T.L. Taylor and Power Gamers}

Sicart's views on instrumental play are influenced by the work of T.L. Taylor (Sicart 2011). Taylor's ethnography of the Massively Multiplayer Online Game (MMO) EverQuest was one of the first to explicitly discuss instrumental play (Taylor 2003, 2006b, 2006a). In EverQuest (EQ) "power gamers" exemplified instrumental play. While described by other players as "too dedicated" and borderline "pathological", the power gamers interviewed by Taylor saw their actions as "reasonable, rational, and pleasurable". Key to their play was the "focus on efficiency and instrumental orientation, dynamic goal setting, commitment to understanding the underlying game systems/structures, and technical \& skill proficiency" (Taylor 2003:302). This form of play and the related focus on efficiency was equated by other players to labour. Simply put, to other players and to outside observers, what power gamers did was closer to work than play. In the context of $E Q$, Taylor argued that the existence of power gamers provided an alternate mode of play 
that ran counter to people's expectations of what fun and play should be. She further notes: "What was striking to me was the willingness of power gamers to go through very hard work to achieve their goals. It was not the activity itself that became the measure of "fun" but the possibility for success that pushed them forward" (2003:303). Taylor thus highlights and problematizes an important dichotomy in game studies dating from Huizinga (1955) and Caillois (1961) that places leisure on one side and labour on the other.

I will make two brief points relating to the legacy left by the progenitors of game studies. The first deals with Roger Caillois' concepts of paidia and ludus and the idea that the complexity of rules in games and the prevalence of rule directed behaviour can be used to classify the forms of play, thus giving a name to the difference that Taylor found between the power gamers and the larger $E Q$ player community. Ludus, in its common usage $^{65}$, is associated with formal games and a reliance on rules to structure the experience (e.g. poker), while paidia is associated with free-form play, without goals or victory conditions (e.g. children playing 'make-believe'). Caillois' definitions of paidia and ludus have been refined by Gonzalo Frasca, who defines ludus as "activity organised under a system of rules that defines a victory or a defeat, a gain or a loss" and paidia as "physical or mental activity which has no immediate useful objective, nor defined objective, and whose only reason to be is based in the pleasure experienced by the player” (Frasca 1999). Following this definition, the rule-bound, goal-oriented behaviour of power gamers is in alignment with ludus, while other forms of free play in $E Q$, such as

65 In-depth examinations of the significance of paidea and ludus can be found here (Frasca 2001; Juul 2005). 
role-playing, can be equated with paidia. This separation between goal oriented and free play, ludus and paidia, aligns with the conflict between instrumental play and roleplaying described by both Marinka Copier (2007) and Joris Dormans (2006). ${ }^{66}$

Grimes and Feenberg adopt Caillois, describing paidia and ludus in terms of occupying two poles on a continuum. Paidia, at one end, describes open-ended, unscripted play whereas ludus describes formalized play that is bound with arbitrary, imperative, and tedious conventions. Caillois argues that as societies modernize, there is a move towards ludus from paidia, from "turbulence to rules" (cited from Grimes and Feenberg 2009:109). Although games are increasingly institutionalized, the established rules are still subject to paidia. This explains, in part, the process of rule-forming and reforming that takes place during the mangle of play, and the dialogue between player and game.

At first glance, paidia and ludus might explain the power gamer phenomenon described by Taylor: power gamers prioritize ludus, rules, and winning conditions. But this brings me to my second point about the legacy of Huizinga and Caillois: the forms of play described by Taylor do not align with the definitions of games and play that are foundational to game studies. Huizinga and Caillois' definitions of games created a dichotomy between work and play. This divorce of 'real' from 'game', 'play' from 'work', 'labour' from 'leisure' traces its game studies ${ }^{67}$ roots to Huizinga's notion of the "magic

66 As a side note, there is the related argument that instrumental play is at odds with social activity (Ducheneaut et al. 2007; Copier 2007) and the assumption that power gamers are only out to help themselves. In counterpoint, both Taylor (2003, 2006b) and Simon, Boudreau and Silverman (2009) highlight the inherent sociality of instrumental play, especially in the context of MMO guilds and the sharing of detailed technical knowledge and strategies on how to play the game.

67 This separation of leisure from labour, of course, is well known beyond game studies, but in the interests of brevity, I am restricting my analysis to Huizinga and Caillois. In regards to Boltanski and Chiappelo (2007), the blurring line between work/play in game studies echos the larger blurring 
circle", which emphasizes a boundary between games and everyday life. The magic circle describes spaces of play that have been marked off as "temporary worlds within the ordinary world, dedicated to the performance of an act apart" (1955:10). Huizinga defines play as a "free activity standing quite consciously outside 'ordinary' life as being 'not serious', but at the same time absorbing the player intensely and utterly. It is an activity connected with no material interest, and no profit can be gained by it” (1955:13). Influenced by the work of Huizinga, Caillois further defined games as being separate from the real world, make-believe, and importantly, unproductive: "creating neither goods, nor wealth, nor new elements of any kind; and, except for the exchange of property among the players, ending in a situation identical to that prevailing at the beginning of the game" (as cited in Salen and Zimmerman 2004:76). The view that play is fundamentally different from and opposed to work is problematized by the serious play of power gamers, the work of productive players, and the realization that sometimes play is boring, tedious, and can look an awful lot like work (case in point: the 'grind' in MMOs to level up, or the repetitive click-to-harvest mechanics in many Facebook games). Returning to Taylor, power gamers and their instrumental play push the boundaries of what constitutes "legitimate" play (2003).

As Taylor's ethnography moves from $E Q$ to World of Warcraft (WoW), an interesting shift occurs in her view of instrumental play. Whereas in $E Q$ instrumental play is a play style freely adopted by a relatively small number of players, in WoW instrumental play dominates, leaving little space for alternate modes of play. This 
analysis is echoed by Sicart, who states that WoW is designed "against creative play, and in favour of instrumental play" (Sicart 2010:26) and Copier, who states that WoW is “coded for instrumental play" (2007:62). While the degree of instrumental play varies according to the player, the spatial and temporal context of the play session, and the game itself, this means that WoW, the world's "most popular MMORPG" (Wikipedia 2011) and the model that all other MMOs and online games are measured against, makes alternative modes of play nearly impossible. This increasing prevalence of instrumental play can also be documented through the growing popularity of social games, and the nascent gamification movement.

What sets Taylor's view of instrumental play apart from Sicart's is that Taylor links the rise of instrumental play to the sociotechnical objects created by players. In Sicart's experiences playing WoW, "Fun was only what the game deemed was fun, and that was largely defined by the actions rewarded by the system and constrained by the rules", thus other modes of creative play were effectively designed out of the game (2010: 26). Sicart situates the growth of instrumental play as top-down process: a result of proceduralist thinking on the part of game designers and academics. But Taylor depicts a bottom-up process - something much more complex and messy: players themselves, and not just designers, have a part in determining and legitimizing 'fun'. In the case of WoW, instrumental play is not only prioritized by the designers of the system and imposed hierarchically, but it is also encouraged and enabled by players, operating in a more networked manner.

Creative play does not necessarily run counter to instrumental play. There is still 
space for creative free play between the rules, as demonstrated by the work of modders. In accordance with the idea of appropriation as a fundamental part of play, Constance Steinkhueler (2006) describes a "mangle of play", where the game that is played by users is not the game that developers originally had in mind, but is rather the outcome of a mangle of production and consumption, human intentions (of both designers and players), material constraints and affordances, broader social norms, cultural practices, and even chance. Yet, even when players appropriate and reshape the game, they often do so in ways that reinforce instrumental play. Of course, it is important to remember that, despite the increased potential for emergent play that comes with online multiplayer contests, designers and the industry as a whole have a vested interest in channelling and shaping play, even emergent creative play, and thus directing it in instrumental ways.

To demonstrate players' roles in promoting instrumental play, Taylor discusses user-created mods, particularly the damage meters and UI mods that are essential tools for high-end play. These mods facilitate and shape collaborative play in guilds, allowing players to assess their own performance in quantifiable terms and allowing for the lateral surveillance of guildmates. For example, damage meters specifically quantify and rank a particular player's contribution to a fight, encouraging processes of ranking and normalization. As Taylor cautions, these tools are imperfect. While seamlessly integrated with the core system, there are flaws in their design. In particular, these mods cannot capture all meaningful player action. This means that player actions that are not amenable to statistical analysis and quantification, such as role-playing ability, are deemed less important. For example, Taylor argues that quantified damage data and statistics work to 
dwarf other aspects of play and participation (2006a:328). Mods become key actors in defining what constitutes 'good play' and thus constructing a particular form of rationalized play. Because of their centrality in guild raiding operations, players that opt out of mods must also opt out of high-level encounters as well. Taylor suggests that because these mods are increasingly mandatory in guilds, they become "emergent coercive systems" (2006a:332) that reshape what play is, and "normalize particular forms of play and action such that alternate modes appear not only unthinkable but downright stupid to many players" (2006a:336). In WoW, nearly every player is now a power gamer, using highly advanced statistical tools to quantify their play experience and determine the most efficient route to success. The valuation of this rational-instrumental approach has permeated online game culture as a whole (Silverman and B. Simon 2009:357).

Mark Silverman and Bart Simon's analysis of Dragon Kill Points (DKP) describes another player created sociotechnical system that allows players to monitor each other, rank contributions, and distribute loot. In this context, DKP systems might be seen as a paradigmatic form of a disciplinary technology. They serve as yet another example of how the creative activities of players (i.e. in this case, those who create systems that regulate DKP) work to promote instrumental play (2009). MMO players are depicted as "hyper-workers" who exhibit an idealized from of perfect rationalization and perfect discipline. At face value:

The basic levelling mechanics of "the grind," power gaming guild structures, and DKP produce what we might call a power gamer subjectivity and power gaming as we understand it today is inseparable from this composition of forces. The combined effect of these is the production of a particular kind of play that resonates with the rationalized and bureaucratized conditions of social life in late modernity. It would be logical to conclude then that power 
gamers are the penultimate player-workers of the information age. (Silverman and B. Simon 2009:373)

Yet, while power gamers produce a social order, it is one much different than those created by modern capitalist institutions.

In contrast to those who argue that games have now become training grounds for work (Yee 2006b; Reeves and Read 2009) Silverman and Simon suggest that this instrumental, hyper-rationalized labour in games is a form of play - it is a critique of hyper-rationalized post-industrial work, rather than a form of post-industrial work. ${ }^{68}$ Power gamers devote significant time and effort to the MMO, but they are not actually producing or consuming anything - they are simply "role playing an ideal form of the rational-economic player character" (2009: 358), and may quit any time they like. Accordingly, because this is all play and not, in fact, work, DKP:

is not a technology of disciplinary power so much as a hyper-technology that extends instrumentality and rationalization to the point of pointlessness. Rationalization implodes with DKP, and the subjectivities of late modernity implode with it. Within the localized subculture of the power gamer every action makes sense, and DKP is simply a pragmatic solution to an increasingly large-scale coordination problem. But the subject produced by this peculiar arrangement of forces has no purchase on the world. (Silverman and B. Simon 2009:374)

Silverman and Simon remind us that games can simply be turned off, along with the instrumental subjectivities and focus on rationality and efficiency. The subjectivity created by the game disappears because it has no physical hold upon the actual body of the player. And so, like the game itself, the subjectivities sit abandoned on the shelf, gathering dust, forgotten by the player.

68 Silverman and Simon argue that, on an institutional level, casual gamers and not power gamers represent the ideal subjects. This is because casual players do not make so many demands upon the game publishers, unlike power gamers. 
Yet, I find myself troubled by Silverman and Simon's provocation, especially when I think of the games that are currently experiencing massive growth in player populations, such as social games. Silverman and Simon suggest that the example of power gamers highlights the the precariousness of disciplinary power in the digital age. I agree with Silverman and Simon that within digital spaces and digital games in particular it is relatively easy to enable hyper-rationalized forms of social organization, especially those predicated upon surveillance and quantification, yet it is much harder to maintain these systems. Players simply drift away and the hold of the game dissipates. However, I believe that something larger is at play here. Yes, power gamers can walk away from the game, abandoning their virtual goods, DKP, and accumulated reputation to migrate to another game. But what does this abandonment mean when more and more of our games prioritize instrumental play? We may abandon the grind of WoW, but as Silverman and Simon acknowledge, this grind and instrumental play predominates most MMOs. We can abandon MMOs altogether in favour of other types of play such as console FPS shooters or farming games on Facebook, but these games are also premised on a hyper-rational instrumental play, characterized by precisely charting one's progress through the game and comparing one's rank to other players. Even in single player games, normalization and rationalization are omnipresent in player rankings; leader-boards, trophies, and achievements. And with the rise of gamification, instrumental play can be found in traditionally non-game spaces such as the classroom and the workspace.

While the power gaming in WoW may represent an extreme instance, instrumental play is a dominant form of play in most digital games. And while power gamers in WoW 
may be a drain on the institutionalized system of the game, consuming resources but producing nothing, I would argue that players are productive. As I previously highlighted (Whitson 2010b), players are increasingly enrolled as producers of content. But more importantly, especially in the F2P model of games and gamification efforts, players ARE the product. They produce reams of information that are commodified and sold. And while power gamers can walk away from $W o W$, it is not so easy to walk way from the growing number of games that are imbricated with our everyday lives such as Facebook games, or games that promise the fulfilment of tangible, rather than virtual, goals (e.g. weight loss, medical health, financial solvency, etc). Furthermore, it is even more difficult to walk away from workplaces and educational systems that are premised on gamification. And so, while the ease of walking away from the instrumental player subjectivity in MMOs may work as a critique of rationalization, I would emphasize that we cannot easily abandon all games to the shelf, and while we may extricate ourselves from specific games, their replacements may create even more hyper-rationalized subjectivities. Sure, many games are "a culturally situated lark, a pastime, a dalliance" (Silverman and B. Simon 2009:374), but as games and game mechanics colonize more and more sectors of our lives, this is increasingly not the case.

\subsection{Grimes and Feenberg's Theory of Ludification}

Sara M. Grimes and Andrew Feenberg, drawing from the latter's theory of instrumentalization (2002), have formulated a critical theory of digital games in order to explain how play practices come to reproduce the processes of rationalization at work 
within capitalist societies. Like Sicart, Taylor, and Silverman and Simon, Grimes and Feenberg problematize the dichotomization of work and play. Because the "rules [of a game] define a play domain with unambiguous measure of success and failure and a clear-cut distinction between strategic and nonstrategic action" games exemplify formally rational systems (Grimes and Feenberg 2009:105). They, like Silverman and Simon, see games as systems of social rationality akin to other modern institutions and emphasize that, due to the nature of play, this rationality works differently than in other bureaucratic organizations. More importantly, Grimes and Feenberg provide a framework to account for how play changes as it becomes rationalized and argue that this rationalization is achieved specifically through the technological mediation of games.

Games have always contained rational qualities such as rules, points systems, etc., but while games may be amenable to becoming systems of social order, they only do so when they are institutionalized on a large scale. Citing the work of social theorists such as Marcuse, Mills and Bourdieu, Grimes and Feenberg suggest that, "as play activities become more organized, even administered, they are increasingly structured by the same values, priorities, skills, and norms that drive the workday" (2009:107). Games, accordingly, are systems of social rationality that operate on three principles: 1) the exchange of equivalents (e.g. players all start the game on equal footing - at level 1); 2) classification and application of rules (both those rules internalized by game system and those rules established by the player community); and 3) optimization of effort and calculation of results (e.g. levelling up, points, the social capital that skilled players accrue, etc.) (Grimes and Feenberg 2009). 
Grimes and Feenberg trace the beginnings of the institutionalization of games to the standardization of the rules that accompanied the professionalization of sport. Professionalization of leisure activities such as baseball encouraged a strict adherence to the formal rules in order to create a homogenous experience for the participant, as well as to allow spectators to follow the game. This homogenization is also essential in order to commodify the experience (e.g. broadcast rights, audience shares, etc.). The creation of a homogenous experience, by necessity, reduces the potential for the spontaneous negotiation of rules that is part of more informal game play.

As games become technologically mediated, commodification is facilitated through increased opportunities to "measure and monitor, package and repackage" the experience (Mosco, as cited by Grimes and Feenberg 2009:108). Online games epitomize the ease of creating highly administered homogenous experiences, as the players' actions can be standardized though the program code; the boundaries of the game are technically mediated and enforced, thus reducing the potential for renegotiation of the rules and limiting player agency to a set of predetermined options. ${ }^{69}$ Citing Taylor, Grimes and Feenberg acknowledge that online games contain space for more collaborative and emergent play experiences, emphasizing that those players with high levels of situated knowledge are able to engage with digital systems of games in unanticipated ways, such as modding. In contrast to the rationalization imposed by corporate owners and administrators of games, Grimes and Feenberg speak of a "democratic rationalization" that can occur when players challenge and restructure the underlying social order of the game. Yet the game code provides the scope and limitations for the vast majority of in-

69 For a more extensive discussion of this, please see (Whitson 2010b, 2010a). 
game activities. While games such as WoW are constructed with flexible user interfaces that allow player-developers to modify and provide core functionalities to the game experience, since "the majority of players do not have the technical expertise required to intervene at this level, most player actions fall firmly within the scope of what is provided by the Blizzard game engine" (Grimes and Feenberg 2009:113). This does not preclude unscripted or emergent behaviour on the part of players, but the ways in which players can appropriate the game environment for a variety of social and creative purposes is often restricted and channelled in certain approved directions by the game designers.

Grimes and Feenberg's theory of ludification describes a series of three transformations that occur when we play a game; transformations that must occur in order for us to move from the undifferentiated practices of everyday life into the magic circle, as depicted by Illustration 4.1 which is reproduced (and added to) below.

\section{Illustration 4.1: Ludification and the rationalization of games} transfomation 1 transfomation 2 transformation 3 transformation 4

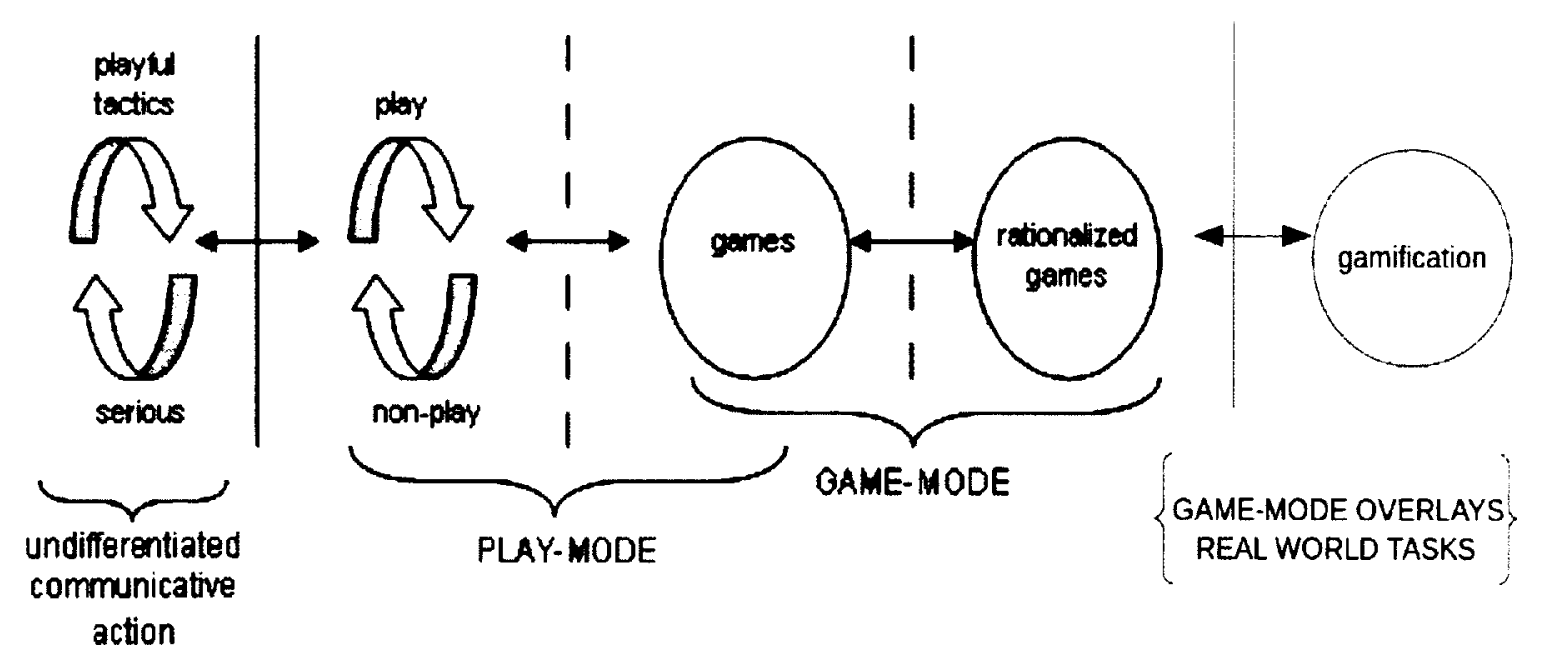

Source: Adapted from (Grimes and Feenberg 2009:110) 
The first transformation is the move from everyday playfulness, which is a momentary and unorganized action that modifies serious contents, to more organized play. This play "is not yet constrained by permanent rules and not fully separated from the world of nonplay, "reality," which threatens to intrude from time to time" (2009:111). The second transformation aligns with the shift from paidia to ludus, where the play is increasingly rule-governed, and the temporal and spatial boundaries of the game become more fixed, delineating what belongs to the game world from what does not. With the move to more ludic play, the player enters a state of heightened reflexivity about their relationship with the game's rules and boundaries. In this sense, play now includes the desire to win, to progress through the levels of the game or outmanoeuvre a competitor, but to also uncover the game's hidden structure, to push against the boundaries of the game and discover where exploits and loopholes may be found. As Grimes and Feenberg put it, here, "to play a game is thus a dual process, one that demands a delicate balance of playing and gaming": a mixture of paidia and ludus (2009:111).

The third transformation describes what happens when games become technologically mediated and commercialized on a large scale. It is a process of rationalization wherein the rational features that are a part of all games, due to their very nature as rule-based systems, become more prominent. In other words, as games increasingly move online and become sites of commerce and profit, the paidiac aspects of play will decrease, while the more ludic aspects will increase to an unprecedented extent. In particular, rational properties such as reflexivity, boundedness, and rule-governedness all are intensified via their technological mediation. "The rules and parameters of the 
game system are programmed into the game code and become ever more tightly enforced and optimized. Play itself becomes subject to increasingly precise forms of measurement and calculation" (2009:111). Yet, player agency is not absent. Emergent behaviour (hacking, modding, player created content, and sociality) operates both inside (e.g. using game scripting tools to create content) and outside of the formal structures of the game (e.g. creative content on fan sites). Many of these activities are 'playful' in that they play with the normal expectations of play, and thus become metaplay: an exaggeration and playing with the boundaries of the game itself.

Building upon Grimes and Feenburg (2009) I argue that the New Spirit of Capitalism and the Projective Cité help create the conditions for a fourth transformation, ${ }^{70}$ a transformation detailed in the previous chapter on gamification. Following the New Spirit of Capitalism, citizens take responsibility for their own self development, a task that is never-ending and not restricted to work hours. As put by Boltanski and Chiapello, in the Projective Cité citizens cannot squander time on pleasures of the "ludic variety", free time must be allocated to seeking information about good projects and kept in reserve to exploit future opportunities - especially in the face of unstable labour markets and job shortages $(2007: 152)$. However, with attempts to gamify life, we see how the playful moods, game mechanics, narrative devices and visual aesthetics of games are increasingly applied to everyday life. In contrast to the second transformation where players move from the undifferentiated practices of everyday life into the magic circle, in the fourth transformation players are asked to suspend their disbelief, overlaying the

70 In the following chapters on the game industry and the rise of social games and metrics, we will see how this works on a an empirical basis (i.e. we will see how the Projective Cite shapes game production processes towards certain ends.) 
magic circle on top of their everyday experiences in the interests of self-mastery through games. Through the fourth transformation, games promise us the pleasures of play and an escape from the pressures of work, school, and family life, while simultaneously offering us routes toward self-improvement. ${ }^{71}$

As games move through this series of transformations, they develop five key properties that enable their transformation into systems of social rationality: reflexivity, boundedness, rule-governedness, precision, and playfulness. Grimes and Feenberg argue that these properties can be established technologically (e.g. designed into the code of the game system) or structurally (e.g. norms, etiquette, legal contracts such as EULAs), but they all must be present in order for the game to operate as a system of social rationality. I will discuss each of these properties in turn, and how they relate to the fourth transformation I propose above and the idea that play can be productive.

\section{Reflexivity:}

As play becomes rationalized, it becomes increasingly self-referential and exclusionary of themes and activities from outside the constructed reality of the play activity or game. The system and structures of the game, along with the player's role, gain in primacy at the expense of an increasingly differentiated "outside" or "real" world. (2009:112)

Reflexivity is characterized by a deep engagement with the system and structures

of the game. In the terms of Raph Koster, it is about 'grokking' the game, understanding the system so well that players become part of it (2005). Players learn how to navigate through virtual spaces via controls until actions become

71 While I reserve judgement on whether these promises of self-improvement actually deliver, this promise fuels the rapid rise of gamification. In cases such as the call centre described in the previous chapter, games are used to mask the lack of real autonomy and creativity by allowing workers more in-game autonomy. 
intuitive. They develop a sense of a game's mechanics and, in doing so, a sense of the range of possible actions. They learn just what it is they must do to earn points, to get ahead, to beat the competition. Through feedback, the game communicates with the player, indicating the route towards the goal. These rewards (e.g. points, levelling up, acquiring virtual goods, unlocking new content) provide players with a template for 'proper' game play, revealing and highlighting the measurement criteria by which players' actions are evaluated. In the case of power gamers, they are so in tune with the workings of the game system that they can manipulate the game in ways that seem impossible to neophyte players.

In the case of gamification and other forms of productive play, when play is super-imposed over everyday tasks, players are reflexive to the game and its rules. This is an ideal technology of governance, in that the pleasures of play and the flow of the game mask how play is specifically constructed to reshape players' worlds outside of the game. Themes and activities extraneous to the game are excluded as players "zone into" the game and real world consequences fall away. The world is seen in game terms, and instrumental rationalities based on progression, competition, and winning, are foregrounded in contrast to preexisting social mores, norms, and values. For example, Facebook games are sites where the values of instrumental play commonly conflict with non-game social norms. Losh (2008) used case studies of popular games to highlight how players may violate social norms about aggression, proximity, and privacy in ways that sacrifice real-world friendships, while Rossi (2009) argued that while Facebook 
games can serve a social bonding function, the design of Facebook games

encourages players to use their social networks as a strategic resource (i.e. treating

friends and family as game tokens).

\section{Boundedness:}

Since play is a differentiated activity, a level of boundedness must always exist in order to distinguish play from the undifferentiated communicative practices of the lifeworld. As games become rationalized, however, the boundaries, in terms of the scope, space, and possibilities for play, become more limiting, well-defined, and self-contained. (2009:112)

In the context of digital games, the boundaries of the system are inscribed in code.

This code creates the environment of the game, supplies it with the laws of physics, and determines the range of possible actions. While emergent behaviour can occur, it must occur within the predefined constraints of the technical system (unless, of course, these constraints are remade through hacking). For the most part, the coded boundaries of the game are hidden behind the graphic user interfaces. Accordingly, the majority of players do not see nor do they directly engage with the lines of code that create the game world within which they play. ${ }^{72}$

This boundedness has important implications for games as governance, in that they map out a territory of government that is bounded in time and space, where all actions are visible, and the range of player activity is predetermined. Following Rose, games in this sense are a form of cartography, exemplifying the ways in which "spaces are made presentable and representable in the hope that they might

72 This chapter is explicitly concerned with the work of authors who explicitly address the instrumentalization of play. More generally, the work of Lawrence Lessig (1999) and Alexander Galloway (2004) addresses how digital technologies create bounded spaces of control. 
become docile and amenable to government". The boundaries of the game and what objects, actions, and people matter are clearly marked: "Salient features are identified and non-salient features are rendered invisible" (N. Rose 1999:36). When games undergo the fourth transformation and become applied to the outside world, this boundedness imposes artificial constraints on users' behaviours. For example, gamifying a call centre results in limiting the range of activities in what is already a highly restrictive workplace. Compelled to use the game dashboard, workers concentrate on fielding as many calls as possible, in order to earn 'treasure'. A number of other activities are supported by the game interface (e.g. lending aid to a co-worker, taking lunch breaks), but other activities (i.e. reporting labour code violations, or unionizing) are not.

\section{Rule-governedness:}

When play is transformed into a game it becomes governed by a specified set of rules and parameters. As games become rationalized, their rule systems become more rigid and comprehensive as they are determined at the technical and institutional level. (2009:112)

Rule-governedness operates in tight conjunction with boundedness and reflexivity. Rules define the game, its limits, and constrain player behaviour accordingly (Juul 2005:32). They describe the formal structure of the game and operate on multiple levels: operational rules are usually synonymous with written rules and are guidelines the player requires in order to play, constitutive rules are the logical mathematical and formal structures that underlie the game, such as code, while implicit rules are the unwritten rules of the game and concern proper 
game behaviour and etiquette (Salen and Zimmerman 2004:130). According to Bernard Suits, game rules prohibit more efficient in favour of less efficient means. ${ }^{73}$ As a result, the challenge of a game is to achieve the goal while working under the constraints of rules. The rules construct the possibility space of the game (i.e. what players can and cannot do) - they are affordances that permit certain actions while prescribing and preventing others. By accepting to play, players consent to the constraints posed by the rules, both those that are written in code, and those that are enforced by other players (norms and community expectations) and handed down by the game publishers (EULAs and Terms of Service).

What makes digital games different from traditional games (e.g. card games and board games) is that the rules are embedded in the hardware and the software of the game, thus freeing players from having to enforce the rules themselves. Digital technology enables the automation of complex procedures, allowing games to be more flexible by storing and restoring different game states, and more complicated by concealing some of the mechanics from players (e.g. physics engines, statistical calculations that compute damages, etc.). Technologically mediated rules are "rigid and precise, and cannot be negotiated or challenged by the average, nonspecialist player" (Grimes and Feenberg 2009:114) meaning that very few people have any ability to change or push back against the rules. The automation and opacity of the complex rules and procedures are at the heart of

73 Suits defines a games as the following: "To play a game is to engage in activity directed toward bringing about a specific state of affairs, using only means permitted by specific rules, where the means permitted by the rules are more limited in scope than they would be in the absence of the rules, and where the sole reason for accepting such limitation is to make possible such activity" (1967). 
governance. Technological systems - even seemingly benign ones like digital games - provide a set of rules, or scripts, encouraging certain uses and interactions and denying others (Winner 1986; Akrich and Latour 1992). For many theorists (Castells 2000; Lessig 1999), what makes computers and software systems such as games important topics of research is that the environments they create are entirely built and dependent upon digital architecture - the coded rules that determine how the software will operate and what functions will be allowed and disallowed. While the opacity of the rules may not matter as much in a game such as Halo, the increasing quantification of our most intimate selves though apps that track our fitness, IQ, sleeping patterns, social interactions, etc, means that more and more of our personal lives are guided by rules that are locked away within the black box of technology, and thus unquestionable. And, as argued above, 'playing at real life' using game rules means that game norms and values (winning, competition, efficiency) are substituted for any preexisting norms and values.

\section{Precision:}

The specification and standardization of a game's rules are accompanied by an increase in precision, which enables measurement and optimization of the game play, in terms of both efforts and results. Like rules, precision leads to a reduction in the scope of what is possible within a game, and transforms play into a quantifiable and predictable set of activities. (2009:112)

Precision operates in conjunction with the collection of metrics and the quantification of player activities. Technologically mediated spaces allow for unprecedented levels of surveillance and quantification, not just in a top down 
manner (e.g. game companies collecting data), but also in terms of lateral surveillance between players, as well as solitary self-directed surveillance. As I have argued elsewhere, digitization allows for previously unimaginable levels of inexpensive data collection, tracking what players do, what they say, where they go and who they talk to in the game space (2010b). This data is easily converted into analyzable forms, such as the damage statistics detailed by Taylor (2006a). The precision of rationalized games allows players to measure their own progress and chart how they compare with others, thus encouraging normalization and acting as objective tools used in social coercion. Similar processes allow for the measurement and comparison of games themselves: indicating how well they compete in the market, determining which games and developers are winning in terms of the most retail sales, players, or advertising revenue.

As further argued in Chapter Nine, the relative ease and low cost of collecting player data greatly impacts the way games are designed. Intricate tracking and data-mining systems allow designers to discover new patterns in behaviour and player preferences, and thus find new ways to create compelling play experiences (or, as in the case of some social games, find new ways to impel player spending). These detailed profiles of player behaviour, especially when linked to their demographic data (e.g. location, age, gender, Facebook page) create a new income stream for the game industry: the commercial sale of these profiles (e.g. user trend reports, advertising, and other marketing initiatives). More importantly, when users consent to gamify their life and experience the fourth transformation, this 
data is then explicitly applied to govern their behaviours outside of game spaces.

\section{Playfulness:}

Playfulness describes the undifferentiated form of play that occurs within everyday communicative practices. Contrary to the imaginative freedom of play, playfulness is characterized by its situatedness within and dependence upon the game system to provide direction, themes, and content. Playfulness can be subversive or reactive, but always functions in direct interaction with the rules, temporality, sequence, and structures of the game. (2009:112)

Playfulness is the reflexive dialogue between the player and the system of rules. In this dialogue, the player may submit to the rules, but more often they push against, creatively appropriate and reshape these rules. Playfulness describes the players' relationship to and negotiation with the social rationality of the game. Sometimes, players may play within the system, but others may play with and against the system. While the rules of the game provide some constraint upon players' freedom, by definition, there is always room for play within these rules, especially in terms of unanticipated emergent play. Many of the player behaviours discussed above are examples of playfulness, from trial-and-error explorations in order to grok the game system, to the development of player mods, to the hyperrationalized, yet unproductive play of power gamers described by Silverman and Simon.

The extent to which playfulness has a role in gamified environments is debatable. While undoubtedly the game system provides the direction, themes, and content that governs and shapes real world activities, the ability of users to push back on or alter the rules of the game are tightly constrained. This is not only due to the 
lack of technical aptitude on users' part, but also because any changes to the rules of the game might endanger the whole enterprise (i.e. the goal of self-mastery and improvement cannot be altered or the whole game is rendered moot). For example, finding loopholes in the points system of a weight-loss app would defeat the purpose for the game, just like cheating and exploits make social leaderboards unintelligible.

The five properties Grimes and Feenberg describe deeply resonate with the themes that emerged from my data collection and interviews. I am therefore following in their tradition, using these five properties as orientating touchstones throughout my chapters. I deeply believe that games are systems of social rationality that operate and should be situated within the larger sociohistorical context of modernity, especially in terms of how shifts toward neoliberal government influence the direction of new media. Like Grimes and Feenberg, I also believe that as games become increasingly technologically mediated, play becomes institutionalized. It becomes rationalized. It becomes commercialized. Increasingly, play is instrumental. It is highly surveilled, quantified, and measured. It operates in service to other higher goals, such as teaching players about world crises, or reducing their fuel consumption, or simply getting them to return more often, invite friends to the game, divulge their personal information, and spend more money. It flows in coherence with the commodification of leisure and the widespread rationalizing processes of modernity.

It is important to clarify that I am not espousing technological determinism here. The production of technology is framed/created within socio-economic contexts and 
value systems (i.e. Spirits of Capitalism) that promote efficiency, productivity, accumulation, etc.. I believe that individual agents have the power to alter and reshape technology in more beneficial ways but often cannot justify their proposed changes in the languages of the current Cités, all of which are oriented toward the accumulation of capital. Accordingly, the following chapters will not focus on play and players, but will look at game developers themselves, the creators of the games that constrain us, emphasizing their attempts to reshape the trajectory of game development and their vocal resistance to many of the trends discussed in the the previous chapters.

On a reflexive note, I am not sure if this is a bad thing. By 'this' I mean the increasing amount of goal-directed play, the growing emphasis on ludus, on rules, and a decrease in the chaotic performance of paidia, the emphasis on charting progress, and the idea that when I play a game I am moving upwards, forwards to some greater goal. I feel productive - that I am making progress - even if I am not. Perhaps this is because, in real life and the Projective Cité, this linear teleological progress is rare (How can you move 'up' or progress in a network?), and rewards and reputation are difficult to assess (Boltanski and Chiapello 2007:92). The vestiges of the Industrial Cité remain, in that I relish the security that comes with knowing and surpassing these preset and linear expectations (i.e. beating the game). The quantification and surveillance that occurs while I play is incidental, as the metrics that come out of it show me how to improve, how to become better, how to excel. I willingly trade my privacy for a sense of self-mastery and control. I am already the perfect neoliberal gamer, eager to discover the route to success. ${ }^{74}$

74 This is a recent revelation that came after a brief chat with Miguel Sicart about what games we were playing. Miguel listed a raft of unfamiliar indie games and board games, including a whole bunch of abusive games (Wilson and Sicart 2009, 2010). I nodded my head sagely when he mentioned a few I recognized. Yet, these games with all their emphasis on paidia, performance, and dialogue between the 
Despite my personal reticence to follow Sicart and lament the loss of paidia ${ }^{75}$ in contemporary games, I do believe that the instrumentalization of play has directly negative consequences for developers and the creation of cultural commodities. In terms of gamification, I am more troubled. Gamification services promise a future where everyday tasks are made more fun yet, when compared to actual practice, this promise rings hollow. Once the leaderboards, charts, and badge systems are stripped away, nothing remains but surveillance machines.

\subsection{Conclusion}

In this chapter, we saw how play is enrolled in New Spirit of Capitalism via ideological shifts that increasingly frame play as productive. These shifts, particularly the growth of instrumental play, mean that games are increasingly used as technologies of governance. While the last two chapters focused on the player and changing notions of play, the following chapters examine game production, using empirical descriptions of the industry to chart the increasing instrumentalization of play, starting with the console sector in Chapter Six, adding other established game sectors such as PC and handheld games in Chapter Eight, and finishing with Casual games in Chapter Nine. The goal of these chapters is to show how the instrumentalization of cultural commodities such as games is

designer and player, are really not what I play for fun. When I head down to my basement, I plug in a Dead Space game, or Borderlands, or Bioshock. I am one of the dumb hordes of players who are simply not interested in sophisticated, meaningful art games. I like to shoot zombies and be rewarded for it. This is the gutter of my reflexivity.

75 I also want to avoid sounding nostalgic for the 'good ole games' of the past. Certainly, the games I played in my youth did not emphasize paidia any more than the ones I play today. My normative stance on games is not retrospective, but looks to the future, hoping that as games become more proficient at shaping human behaviour, the direction this shaping takes and the motivations behind it become more transparent to players. 
rooted in the economic models of their production. Just as Sicart and Taylor argue that games cannot be analyzed in isolation; that analysis should draw attention to how players interact with, appropriate, and re-shape games, I argue that discussions of games should incorporate much deeper reflection on how games are made, who makes them, and the processes that shape the production of games, as these reflections are key elements in understanding the rise of instrumental play itself. The next chapter sets the stage for the rest of the thesis, highlighting why developers are an important avenue of study. 


\section{Chapter 5. Researching Game Development}

At this point in the thesis, I shift my approach. In Chapters One and Two, I introduced current shifts in the digital game industry, arguing that these shifts can be explained by the shift to New Spirit of Capitalism, and the adoption of rationalities rooted in the Projective Cité and networked logics. In Chapter Three, I used the example of gamification to show how gamifying everyday life is an idealized model of the New Spirit of Capitalism, as well as a paradigm example of governing through freedom. In Chapter Four, I introduced the concept of instrumental play and the rationalization of games, showing how the ideological revision in thinking that games can be productive is necessary step in enrolling games as tools of the New Spirit of Capitalism. In the remaining chapters I focus on game development itself. I examine the socio-economic forces influencing the production of games, highlighting how the New Spirit of Capitalism operates on the the ground. Importantly, I show how current development practices alter the New Spirit of Capitalism proposed by Boltanski and Chiapello (2007, 2005). This chapter situates this shift in approach, first arguing that developers are an important - yet neglected - site of study, and then outlining my research methodology.

\subsection{The Paucity of Developer-Centric Research}

In academic studies of the game industry, developers are largely invisible. With the exception of critical political economy research, innovation and organizational studies that focus on managing the creative process, and a few rare ethnographies, considerations of game developers are absent from game scholarship. The industry itself is often 
simplified into a monolithic entity and the foundations of game production are ignored in favour of analyzing play and players, or glossed over and oversimplified. This is not to say that game studies is not interested in developers. Many game studies academics, such as Ian Bogost, create games and have close ties with the industry. Still more, such as Jesper Juul and T.L. Taylor, interview game developers in the service of player-centric research. Others, including myself, have referred to the game industry in terms of a capitalistic force that opposes players, for example, lodging lawsuits against modders (Jenkins 2006) and endorsing highly constrictive End User Licensing Agreements and other terms of service (Kücklich 2009; Whitson and Doyle 2008; Humphreys 2007). Sometimes, this monolithic industry is depicted as working in alignment with players, channelling their creativity (Banks 2009; Humphreys 2008; Taylor 2007). However, the industry's underlying interest for doing so is economic, and market interests ultimately shape spaces of play. Depicting the true complexity and heterogeneity of the game developers and the game industry is sacrificed in favour of simplified visions of player exploitation and corporate dominance.

The game industry, and developers themselves, are far from monolithic.

Following Jesse Schell, I use the term "game developer" to refer to "anyone who has any involvement with the creation of the game at all" (2008:xxv). This includes engineers, animators, writers, designers, programmers, modellers, and musicians. Tschang (2005) separates game development into three components: programming code, game design, and creating content. These three components are interdependent, working together to provide a cohesive game experience. Kerr (2006) and O'Donnell (2008) both use a 
similar disciplinary structure, separating engineers (programmers), from designers, from artists (content). However, they add a fourth category: management. The roles of producers and other management personnel are important because they must unite these dispersed disciplines and harness their creativity in order to produce a marketable game. Accordingly, game developers as such do not reflect one homogenous community, but rather reflect smaller communities that are separated according to discipline and must work together in order to develop a cohesive game.

The lack of research on developers is largely attributable to issues of access. Discussions about the social context and complexity of the game industry are often censored by researchers in exchange for data and access to their intended sites of study. Game development is an inherently secretive process (see Nieborg 2011; O’Donnell 2008). As a researcher, getting access to this network is in itself difficult and is negotiated within an atmosphere of distrust of academics. Even if access is secured ${ }^{76}$, revealing details about game development becomes an impossible endeavour due to non-disclosure agreements. For example, designer-academic Aki Järvinen explains below why he is no longer writing a book on social game design, or even blogging about the topic:

When I see e.g. blog writings about social games from independent sources, I definitely identify how I could produce text from a similar perspective (and I have) but, in the same breath, I also recognize that the perspective is at least a step or two removed from the daily dilemmas of working in the social games industry: e.g. product metrics, company processes, what we hear from our Facebook liaisons, and so on. Another tangential factor to the problem has to

76 This access if often negotiated in terms of an exchange of services. For example, O'Donnell (2008) and Banks (2002) worked at the studios they were researching. Nieborg gathered his data in context of interviewing developers for industry publications. I would also suggest that gender plays a significant role in access to data. As a female interested in the socio-economics of the industry (and not someone positioning themselves as a potential employee), I was relatively exotic. I had fewer difficulties finding interviewees in face-to-face industry settings, such as developer conferences or IDGA sessions. This access was also facilitated by a well-placed industry contact. 
do with non-disclosure issues: writing a book in a highly competitive space from an inside position just is not possible without either breaking such clauses - which I have no intention of doing - or making compromises which, in the end, take most of the sting out of the substance, or the secret away from the sauce, so to speak. (Järvinen 2011)

There is a clear 'inside' and an 'outside' to game development, and most often academics especially those in the social sciences and humanities - are left standing on the outside.

When granted inside access, academics are effectively muzzled by the need to anonymize their sources. This is due to numerous industry-side factors including: Non Disclosure Agreements (NDAs); worries about leaking details of release dates, budgets, and technologies to competitors; fears that critiques of manufacturers, publishers, and distributors might compromise future partnerships; and, concern that details of budgets and waning profits might discourage potential investors and employees. What this means is even the most general details, such as the name of the studio or the type of game created, must be obscured and hidden in published work. From a legal and an ethical perspective, this anonymization makes sense for academic researchers. However, if game studies is to effectively engage with game development proper, this anonymity is detrimental. In the game industry, game credits (i.e. the listing of games one has worked on) are cultural capital. They are essential determinants of reputation. On a more general level, game developers may come from heterogeneous backgrounds, but they are united by the lingua franca of development - the references to games, game mechanics, play styles, genres, legendary and infamous developers, etc. Not being able to name interview subjects, or even the title of the game in question, means that the credibility of interview data is devalued. More importantly, contextual cues, such as whether the person is a 
graphic artist or lead designer, how long they have worked in the industry, what games they have contributed to, and allusions to the history of games must be omitted.

Another issue related to the anonymization of data in exchange for access and the associated loss of contextual cues is the inherent difficulty in disentangling different conversations, viewpoints and interests. This leads to the homogeneity of developer discourses in academic research discussed above. Game development is not only characterized by an inside/outside, it is also structured into different sectors according to the form and genre of games produced (e.g. console, social, serious games, etc.) and into different communities according to the speakers' affiliation (e.g. product manager, chief technical officer, business analyst, lead level designer, etc.). Quite often, these narratives connect and cross-pollinate and this is where interested parties can track the emergence of trends. For example, using non-anonymous publicly available interview data over a period of three years, I charted the proliferation of business-driven metrics, tracking its emergence from the fringes of the industry in social games, noting the sectors with the most vocal resistance (e.g. indie and core developers) and then tracing the gradual adaptation to new innovations as they are refined and once-critics become vocal converts. In this sense, the most meaningful findings of my thesis were rooted in the dialectics of industry change, as trends such as metrics went through stages of thesis, antithesis and synthesis, most often framed in terms of Core vs Casual and creativity vs constraint, but also framed in terms of the increasing rationalization of game development. 


\subsection{Methodology and The New Spirit of Capitalism}

My methodological approach echoes that of Boltanski and Chiapello (2007), although with some important alterations. Like myself, Boltanski and Chiapello are ultimately interested in the ways in which people engage in action, their justifications, and the meaning they give to their actions. To this effect, they look at how management discourse sensitizes individuals - especially managers and engineers - and enrols them into capitalism. In order to be successful, the models purposed by these management discourses must coincide with these peoples' moral experiences of daily life, and suggest models of action they can grasp (Boltanski and Chiapello 2007:14).

Management discourse provides managers with self-evident reasons for the right actions. It is:

[A] discourse legitimating these actions; encouraging prospects for individual development; the chance for people to project themselves into a future that was restructured in line with the new rules of the game; and the suggestion of new models of reproduction...and upward social mobility ... . (Boltanski and Chiapello 2007:57)

Boltanski and Chiapello found that as Cités change and new Spirits of Capitalism become ascendant in the workplace, management discourse changes as well, providing managers with new answers to the demands for justification. Thus, they focus their analysis on management texts in the 1960's and 1990's in order to chart changes in management discourse over time. Management literature provides practical recipes for improving productivity, and also has a high moral tone, in terms of offering normative judgements and prescriptions for success. It demonstrates:

[H]ow the prescribed way of making profit might be desirable, interesting, exciting, innovative or commendable. It cannot stop at economic motives and 
incentives. It must also be based on normative aims, taking into account not only personal aspirations to security and autonomy, but also the way these aspirations can be attached to a more general orientation to the common good. (Boltanski and Chiapello 2007:58)

During each time period of their study, management discourse provides a coherent accounting of what problems exist, the proposed solutions, and what is being rejected. It provides managers with rules of conduct and guides for action to make their work intelligible, and confers meaning to work which would otherwise seem arbitrary and ad hoc (Boltanski and Chiapello 2007:103).

If I had wanted to replicate Boltanski and Chiapello's study, there are many management texts dedicated to software development. In particular, The Lean Startup (Ries 2011), was a commonly recommended text at the game developer conferences and functions that I attended. Agile and Lean development methods, in particular, align very closely with Projective modes of thinking (see M. Poppendieck and T. Poppendieck 2009). ${ }^{77}$ However, I took a different approach which allowed me to avoid two key criticisms of Boltanski and Chiapello's methodology and their reliance on management texts as a primary data source. The first criticism alleges that management texts may have little influence on workplace realities because their lessons do not trickle down to individual workers. ${ }^{78}$ The second criticism of management texts is that they describe

77 For example, Mary and Tom Poppendieck begin their most recent book with an anecdote of a bank in Sweden and the man who made it profitable. It reads as a paradigm example of the the New Spirit of Capitalism: "Wallander felt that central control got in the way of the people closest to customers, slowed down their reaction time, and stifled their creativity. So decision-making authority devolved to branch managers; staff groups were dramatically reduced in number and size, and those that remained had to sell their services to the branches. ...Jan Wallander understood that the most effective, responsive organization is one where small unit leaders make local decisions. He devised an organizational structure, governance approach, and culture that reliably engaged the creativity and dedication of knowledge workers at hundreds of local branches" (2009:xiv).

78 My own conversations with software developers are riddled with references to management texts and the software development methods these texts recommend. These personal anecdotal accounts seem to run counter to this first criticism, evidencing that software developers are very aware of management 
idealized workplaces that do not align with reality. Boltanski and Chiapello address the latter criticism by arguing that management literature is important precisely because it has a prescriptive, moral tone. It is a normative literature, stating what should be the case, rather than being an exhaustive account of what is really the case. Management literature is thus a way to examine sense-making discourses.

However, I would argue that - in the case of the game industry - management literature is not the only way to expose these sense-making discourses. In fact, the discourse that looks to define the values, the justifications, and the boundaries of the game industry is more clearly exhibited in the blogs, news sites, business reports, developer magazines, and conferences that unite game developers. This is because this discourse does not come from management professionals (like academics, another set of outsiders), but from inside - from industry visionaries and broadcasters of developer culture, morals, and norms. Instead of idealized management case studies and prescriptions, developer discourses present a much messier, conflicted vision of the New Spirit of Capitalism. By looking at developer discourses, we not only see how the values and norms of the Projective Cité are validated and disseminated, but we also see how modes of production that are endorsed by the New Spirit of Capitalism are critiqued, reworked and adapted to fit the heterogeneous needs of those in the industry.

In order to establish general baseline for industry discourse, I performed twelve formal and informal interviews. This sample included representatives from a number of development sectors, including mobile games, downloadable console games, console and PC games, and advergames. My interview subjects came from a wide array of literature and trends. 
backgrounds and included co-founders of game studios, a creative director, general manager, engineering head, programmer, lead designer, indie developer, and those in marketing and publishing. They were all male.

I initially solicited interviewees via public listservs and cold-call emails. Due to the poor response, I tried a different tactic. I contacted potential interviewees ${ }^{79}$ at developer conferences, and asked them face-to-face if they would mind being interviewed. This tactic was much more successful. Interviews took place in a public setting, either in the staff rooms of their game development companies or at public cafes and restaurants. The interviews generally lasted from one to two hours each, with a few lasting longer. I drew from an interview guide (see Appendix B) to provide a starting point for the interview, but generally allowed the conversation to flow in an unstructured way. Allowing for deviation from the interview guide allowed me to adapt each interview to the respective positions of my interview subjects. ${ }^{80}$

I initially intended to use my interviews data much more directly and centrally than I did. However, given the small development community in my interview area, I could not guarantee anonymity. Even making reference to the form of games (e.g. downloadable console game or a iOS game) would identify the studio in question. Since much of my content dealt with potentially controversial issues such as oppressive relationships between developers, publishers, and manufacturers, and thus could potentially damage a studio's business partnerships, I decided not to include quotes from

79 I did not attempt to randomize my sample, but rather solicited a representative cross-section of game industry workers. Thus I was able to gather interview data from all game sectors and from all levels of the development hierarchy.

80 For example, when talking to an executive from a console manufacturer, I did not ask about personal experiences of crunch, but did ask about the industry's perspective on crunch and undesirable working conditions. 
my interview transcripts. I used my anonymous interviewees as informants. Their initial narratives of industry change and tensions directed my focus for the rest of my data collection, telling me what was important to look for, and perhaps more importantly, who was important to listen to. For example, a prominent theme in my interviews was the conflictual relationship between manufacturers, publishers, and Core developers. This theme directed my investigations, becoming the driving force behind Chapter Six.

In short, I used these initial interviews as a barometer, telling me what game developers were currently concerned about, what they love about their work, what fears they have for the future, and where they think the industry is going. I then turned to other qualitative data (i.e. the discourses of developer conferences, news sites, blogs, etc.) to follow these themes, linking the local experiences of developers that I talked to to much larger trends in the development community.

Besides these initial interviews, my research methodology also differs from Boltanski and Chiapello's in terms of how I locate relevant occupational discourses. Boltanski and Chiapello are interested in tracking changes in management discourse, comparing how these discourses changed from one Spirit of Capitalism to the next. In order to do so, they collected representative samples of management texts from both time periods (1959-1969 to 1989-1994), resulting in 1000 pages of text that was subjected to analysis. In particular, they searched for "authors' concerns, the solutions they proposed to the problems of their period, the imagined they offered of the inherited forms they declared to be outdated, and the various arguments advanced to effect the conversion of their readers" (Boltanski and Chiapello 2007:60). I am also interested in these concerns 
and solutions, but look at one specific time period (2009 - 2011) to see how The New Spirit of Capitalism - and the projective justificatory regime that supports it - adapts in the face of critique, and how these adaptations impact the work of game developers and how games are produced. Ultimately, I investigate what the New Spirit of Capitalism looks like at ground zero of the game industry.

To do this, instead of relying on management literature, I use both physical and digital artifacts, such as blogs, news sites, business reports, developer magazines, and conferences to trace and track themes prevalent in my interviews, particularly the instrumentalization of play in game development, the shifting economic landscape, and other problems identified by the authors (e.g. increasing competition, smaller margins of profit, new economic models etc.), as well as potential solutions. Beyond the initial interviews that directed my gaze to prominent topics, themes and keywords, online developer resources such as news sites and developer blogs provided a large source of my data. These sites are amenable to cataloguing, coding for trends, and archiving snapshots, allowing for a more structured content analysis. The collected resources represent a number of perspectives including industry news sites, more specialized sites catering to smaller game sectors (e.g. indie games, social games, etc.), market analysis sites, fan sites, and a number of individual developer/designer blogs.

From August 2009 to December 2011, I used a desktop news aggregator, Liferea Feed Reader, in order to systematically scrape the feeds of approximately thirty popular developer sites, such as Gamasutra.com, Gamesindustry.biz, and Develop-online.net, which are detailed further in Appendix C. This number fluctuated as some sites became 
inactive, others amalgamated, and new ones appeared. On average, each weekday I

reviewed 200 new items, a process which generally took two to four hours depending on

the content. Over the course of two and half years, this meant I reviewed over 130,000 items. $^{81}$

I focused on cataloguing developers' opinions of industry change and what directions they saw the industry moving towards. Divisive topics, including Core vs Casual, cloning vs creativity in game design, and debates on ethical game design, were particularly important as they revealed developers' values and norms, as well as their fears of the future. Pertinent items were then archived by taking a snapshot and coded for themes using the research and annotation system, Zotero, and detailed research notes were appended. ${ }^{82}$ During this period I archived approximately 1000 articles (960) and subjected them to further analysis. I supplemented this data in a number of ways, including attending developer conferences, compiling economic statistics, and analysing game postmortems.

Developer conferences provide additional rich data about upheavals in the game industry and the tense relationships between development studios and publishers, console manufacturers, and retail distributors. They are indicators of general industry sentiment,

81 Yes, this is a much larger data set than that of Boltanski and Chiapello. In contrast to their methodology, I did not subject everything to a second analysis using analytical software and instead relied on the sheer number of articles to find a saturation point and validate my findings. This kept the scope of the project to a manageable size.

82 To capture and document industry trends, I created new codes as I went along. These codes were organized under three categories of focus: game design and development, industry trends, and social impact of games. Representative codes in the cluster on game design and development included: democratization of design, use of psychology, design innovations, techniques specific to sector, etc. Representative codes in the industry cluster included: growth of sector, working conditions, developer culture, death of sector, industry tensions, as well as many codes dedicated to tracking statistics such as revenues for individual games and sectors. Representative codes in the games cluster looked at larger social trends and included gender, governance, emotion, health, instrumental play, learning, violence, and surveillance, to name a few. 
and provide material that is not present in more formal sources such as news sites. Developers are much more forthright when addressing an audience of fellow developers, commonly discussing economic numbers, or 'rants' on subjects that would not otherwise reach a sociologist's ears. I attended MIGS (The Montreal International Game Summit) in both 2009 and 2010, and the Ottawa Game Conference in 2012. All provided networking opportunities that precipitated many informal discussions about emergent trends in game development. The GDC (Game Developers Conference) held annually in San Francisco is the premier conference for developers, with a number of off-shoot GDCs occurring in Europe, China, and Austin, Texas. Video and audio recordings as well as slides of GDC talks are archived and accessed through the GDC Vault, allowing me to chart the sudden emergence of trends such as social games, and the increasing number of discussions about how metrics are applied in practice and their potential to make the game industry more profitable.

To examine the economic models and production processes of the game industry, I drew from a number of statistical sources. Unbiased projections on the size and growth of the digital game industry were difficult to find (Kerr 2006). I relied on a number of sources in order to preserve validity, including the Entertainment Software Association, the National Purchase Diary, the International Game Developers' Association, and economic projections published by Game Developer Magazine. Following Petrillo et al. (2009), I also performed a content analysis of Game Developer postmortems from 20052010, using their categorizations to code the content and highlight common constraints in game development, as well as successful coping strategies. This content analysis model 
counts how often certain problems recur, as well as charting teams sizes, development times, platforms, etc., as well as changes in what types of games are represented (e.g. the recent inclusion of digitally distributed games), and how game analytics and other modes of feedback are used in the development process. This postmortem data directly fed into Table 8.1 in Chapter Eight, and provided essential data on how team size, development processes, technologies, and production schedules according to the type of game made. ${ }^{83}$ Beyond the initial face-to-face interviews and sessions at developer conferences, the bulk of my research material was found online. In a strange twist while critiquing data-mining and surveillance in the game industry, I employed these self-same methods. Following Latour, I note that the digitization and archiving of game industry discourses in such detail online fundamentally changes the nature of social science research, allowing me to precisely chart, quantify, and verify what were previously seen as unverifiable rumours and unsubstantiated intuitive leaps:

In other words, the former distinction between the circulation of facts and the dissemination of opinions has been erased in such a way that they are both graduating to the same type of visibility - not a small advantage if we wish to disentangle the mixture of facts and opinions that has become our usual diet of information. (Latour 2007)

By (obsessively) tracking changing industry discourses over time, I was able to follow different narratives in the game industry, tracing the epigenesis of game industry "rumours" and "fads" until they became "scientific fact" (Latour 2007), as well as tracing the opinions of experts as they change over time (e.g. the gradual acceptance of social games was one of the best examples of this). By contrasting different industry discourses,

$83 \mathrm{My}$ findings from the postmortem content analysis are reserved for future publications, for both space considerations and in the interests of keeping the themes of this thesis as coherent as possible. However, many of the descriptions of working conditions, crunch, and constraints in development cited in the rest of this thesis come directly from postmortems. 
I was able to document industry-wide shifts among different game sectors - a process that would not have be possible via more traditional ethnographic methodologies such as participant observation.

In each of the following chapters, I treat my primary sources as experts and cite them just as I cite my academic sources. Readers interested in tracing my arguments back to the primary source material to replicate my arguments are thus able to do so. In Appendix C, I provide further detail about my online sources, such as a complete list of the sites I followed. To disentangle primary from secondary sources, I created two separate bibliographies. The first, located in Appendix D, references all of the primary data I quote, while the second, located in the Bibliography section, references all of the sources (both academic and industry) I drew upon for my industry overviews, literature reviews, and analysis. Each of the following chapters includes a paragraph detailing how my interview data informed my analysis and how primary sources are used to build my arguments.

\subsection{Interpretation}

From my initial data set of over 130,000 items, I used discourse analysis to further examine the 960 articles that corresponded to the themes of industry upheaval and change arising from my initial interviews. In the context of this thesis, discourse is defined as "the way in which language and other forms of social semiotics not merely convey social experience, but play some major part in constituting social subjects...their relations, and the field in which they exist" (Purvis and Hunt 1993:474). Discourses impose 
frameworks that limit what can be experienced and how experiences are interpreted, which then influence the actions of individuals. In terms of the game industry and the New Spirit of Capitalism, institutional discourses allow certain things to be said while impeding other things, thus affecting the range of possible understandings of, and responses to, emergent trends in the industry.

The symbolic order of the New Spirit of Capitalism is reproduced via game industry discourses. These discourses, whether they are blog posts, industry analysis, or interviews, serve multiple functions. These primary data sources are structures of knowledge and storied ways of knowing that build and reinforce the normative structures of game developers, reflecting the justificatory regimes of society (i.e. Cités) more generally. For example, blog posts and a large proportion of articles on game developer sites are first person perspectives that share the experience of a particular group (in this case, a development studio) so that others may know life as they know it. They are a teaching tool and parable from which other developers can learn, signposting what success looks like in the industry and the preferred routes for achieving it. Importantly, they also afford students, players, and academics a rare glimpse at what developers' life is really like. ${ }^{84}$

The context in which these discourses are written (e.g. the teller, the intended audience, and their respective relationships) are as important as the actual content. For example, in postmortems, events, successes, and failures, are carefully selected, compressed, shaped, recreated and reconstructed. A postmortem published in Game Developer is not the same as a narrative told by the same author and reporting the same 84 The answer to this is: nasty, brutish, and short. 
events, but told in private conversation among peers. Not surprisingly, postmortems of the same game, look vastly different depending on the author, whether they are written by an executive producer, a programmer, a CEO, or lead designer.

The occupation of the writer and the context in which they are writing frame how they represent trends to their audience. For example, financial consultants frame social games much differently than indie developers. And console developers present very different views on the future of console games dependant on whether they are speaking to the general public (i.e. fans and investors) in a high profile interview versus whether they are speaking to a closed room of other developers at a conference such as MIGS. I found myself tuning into and recognizing particular voices as 'experts'.$^{85}$ Some voices recurred simply due to the sheer quantity of their writing, especially for games journalists like Eric Caoili, James Brightman, and Rob Crossley. Other journalists such as Leigh Alexander and Rob Fahey and many others became recurring characters because of their insightful editorials or engaging interviews with notable industry figures. ${ }^{86}$ Developers who reflected on their design processes as well as larger industry issues, such as Brenda Braithwaite, Daniel Cook, and Raph Koster, featured heavily in my analysis, which was also influenced less directly by the work of academic practitioners such as Ian Bogost and Sebastian Deterding. Industry consultants such as Tadhg Kelly and Nicolas Lovell provided pragmatic and often controversial counterpoints to these narratives, offering advice to developers and showing that game development is as much about business strategy as it is about creativity and play.

85 This list is not exhaustive, but serves to demonstrate the different perspectives that fed into and tempered my analysis of industry trends.

86 For example, the in-depth interviews and articles from Matt Martin, Alec Meer, Kris Graft, and Christian Nutt provided a significant amount of research material. 
In attending to the discourses of experts who are widely respected by other game developers, following Boltanski and Chiapello, I am attending to the "great men" ${ }^{\text {87 }}$ of the industry - the visionaries that give the industry shape, articulate norms, and provide templates for success. In each Cité a person's worth is assessed through a legitimate process and in terms of a given standard, thus contributing to their "greatness" (Boltanski and Chiapello 2005:167). Great men strongly embody the Cité's values. In the Industrial Cité greatness is based on efficiency and professional abilities, where in the Civic Cité, greatness is defined by those who express the collective will. In the Projective (i.e. networked) Cité, greatness resides in those who have the largest number of social ties, those who are adaptable, flexible, and work to connect other people together in productive ways. The great man of the Projective Cité is the communicator, the networker ${ }^{88}$, and thus, consequently, the author of the blog posts, news articles, and developer presentations, that feature in my analysis. They define the values of the game industry, unite developers, and - in text - represent the voices of the industry.

Writing on the game industry works to solidify developer culture, not only serving in a training context but also, following Cortazzi, used to entertain, "to express rapport and solidarity, to ratify group membership, to convey collective values and a sense of history and progress" (2007:387). The social transmission of experience through online sources serves an institutional role in promoting the continuity and reproduction of

87 I am directly referring to a gender imbalance here. With few exceptions, the great visionaries of the game industry are male.

88 Boltanski and Chiapello define the great man as a network extender, putting others into contact with each others. However, great men ultimately work to assure the health of the network and often make personal sacrifices to do so (see Boltanski and Chiapello 2007:122). In contrast, the networker is selfish and focused on self promotion, exploiting the work of others for their own gain (Boltanski and Chiapello 2007:356). Networkers thus do not positively contribute to the growth and development of healthy networks. 
developer communities and cultures. ${ }^{89}$ Accordingly, reading online sources as ideological discourses, rather than simply objective accounts of the development process, emphasizes their role as creating and reinforcing developer culture, and defining the boundaries of what constitutes the game industry, as well as where its future lies. Each of my data sources emphasized different contexts and solidified the industry's boundaries in different ways. Yet, the future they depicted was strikingly similar. As we will see in the next chapters, this future lies in the Instrumentalization of Play, in terms playing games as well as creating them.

\subsection{Conclusion}

This chapter addressed why developers are an important site of study, despite the barriers to academic research. It also proposed a methodology for capturing and analyzing heterogeneous game industry discourses. The next chapters untangle these different discourses, showing different sectors of the game industry operate according the New Spirit of Capitalism. As well shall see, emerging sectors are much more agile, adapting easily to the Projective Cité, while the console sector - in particular - struggles to keep up.

89 Tangentially, this also explains why the same issues keep recurring in postmortems, despite the broad range of games represented. Postmortems are a performance of developer culture. Accordingly, it is not surprising that that same morals, the same lessons are continually repeated, as they serve a boundarymaking function. 


\section{Part II.}

The Game Development Landscape 


\section{Chapter 6. Console Developers: Cogs in a broken machine}

In the first half of this thesis, I introduced the New Spirit of Capitalism and - with broad theoretical strokes - started making links to both games and the game industry. I also introduced the Instrumentalization of Play, arguing that play is increasingly seen as productive and that games are rapidly becoming governable spaces. Both of these shifts are rooted in the New Spirit of Capitalism, and the blurring line between work and play in the Projective Cité. However, digital games cannot be understood without attention to the economic systems from which they emerge and the political, social and cultural contexts in which they are produced and consumed. Accordingly, in the next few chapters, I take a more descriptive empirical approach, detailing how the Instrumentalization of Play is embedded in the economic and cultural structures of the game industry.

In 2006, Kerr valuated the global games software industry somewhere between $\$ 18$ and 21 billion (2006:49) ${ }^{90}$ Console games dominated the industry, accounting for 57 - 78\% of all global game software sales (2006:52). Half a decade later, the console industry is collapsing under its own weight. As put by Rob Fahey, writing in response to the demise of a number of high-profile development studios:

In a nutshell - developing top-tier console games has never been more expensive, and it's never been more risky. As such, it's never been less attractive to publishers, who find themselves forced into the position of funding individual projects with cash backing that used to fund a whole title catalogue, and then chewing their nails as they wait to see if they're ever going to see any of that cash again.

It's not that the games business hasn't always been hit-driven and hence risky - it has, of course. It's just that the gambles are getting bigger and bigger, and

90 She used multiple sources for her figure, noting the difficulty of acquiring independent and affordable data to corroborate these reports. 
the odds are getting worse. Moreover, even as game software budgets rise, sales have flat-lined (R. Fahey 2011b).

Long dominant, the console industry has become a lumbering dinosaur struggling to survive while developers on the margins (e.g. indies) and 'outsiders' (e.g. social games, gamification, and mobile game developers) are experiencing growth and financial success. This expansion is largely result of new economic models premised on digital distribution and the shift from games as a retail product to games as a service. The console industry has become overly specialized and is unable to shift quickly enough to keep up with its more agile competitors. Rumours of its collapse abound (R. Fahey 2012).

This chapter accordingly focuses on the struggles of the console industry. It reviews how console games are made, the role of developers in making games, and the instrumental pressures that shape what forms of games are ultimately published. It explains why the console industry is contracting in the face of massive upheavals. The console industry retains many features of the Second Spirit of Capitalism ${ }^{91}$ while emerging sectors embrace the New Spirit, and are much more agile, adaptable, and heterogeneous in style. I will start then, at the beginning, with a brief overview how console games are made. Next, I will detail the relationship between developers, publishers, and console manufacturers. Before doing so, however, I will make the important parallels between competing Spirits of Capitalism and the Core and Casual industry divides, contextualizing the collapse of consoles in the wake of the Casual

91 This is not to say the console industry lacks any ties to the New Spirit of Capitalism. In fact, it embraces many hallmarks of New Spirit businesses - such as an increased reliance on outsourcing, and an emphasis on the role of the 'network' in it's organizational structure (see O'Donnell for more on the network trope). However, the argument here is that the industry has not changed enough in that critiques of the Second Spirit of Capitalism can still be applied to the traditional console industry. 
Revolution.

The direction for this chapter came directly from my interviews with developers. A number of these developers struggled to secure contracts with publishers and manufacturers and were dissatisfied with how little creative autonomy they retained over their games. The few royalties they received after profits were divided between retailers, distributors, manufacturers, and publishers were never enough to fund future development, so they continually began the process of securing publisher funding all over again, trading away their intellectual property in order to keep their studios running. My motivation for writing this chapter was to reveal the structural constraints that cripple traditional game development studios, and how alternative production models are an appealing contrast.

\subsection{Core vs Casual}

Game industry discussions commonly focus on two archetypes of games: Core (hardcore) and Casual. This over-simplistic dichotomy frames much of the discussion of current industry trends, with traditional console and PC production methods associated with "Core", while emerging trends are associated with the rise of "Casual". While the boundaries between the two are porous and depend on the context in which a game is played, it is useful to further define and untangle these terms. As a first clarification, I use "Casual" (capital "C") to refer to all casual, social and mobile game sectors, while "casual" (lower case "c") refers to much smaller, specific subset of Casual games, further detailed in Chapters Eight and Nine. 
No hard rules delineate Core from Casual, but there are important differences between how people play games, as well as why they play. The appeal and accessibility of Casual games can be conceptualized using five attributes outlined in Jesper Juul's book, The Casual Revolution (2010), the first being Fiction. Casual games, in comparison to Core games, have emotionally positive fictions and are located in non-threatening settings (e.g. diners and cheery fantasy realms) as opposed to the violent and conceptually dark settings of most traditional games (e.g. dystopian war-torn cityscapes and outer space). Secondly, Casual games prioritize Usability. Casual games "can be played in short time bursts, and generally do not require an intimate knowledge of video game history in order to play" (Juul 2010:5). In addition, Casual games are easily Interruptible. They demand a shorter time commitment from the player and can be played in short bursts of time, whereas Core games, such as the Final Fantasy franchise, require considerable time commitments in order to complete. Another difference between Casual and Core games is in their level of Difficulty and Punishment. While Core games punish failure (e.g. the player must replay the level until they pass it), Casual games generally have lower difficulty levels and exhibit lenient punishments. Finally, Casual games differ from Core games in their level of visual and aural feedback, which is described in terms of Juiciness. This juiciness takes the form of excessive positive feedback for every successful action the player performs.

While game studies scholars recognize the inadequacies of using static descriptors such as Casual and Core to define games that are more fluid depending on the context of play, in practice (both in academia and actual game development), Core most often refers 
to mainstream console and PC games such as First Person Shooters (FPS), massivelymultiplayer online games (MMOs), and big-budget role-playing games (RPGs) (Consalvo 2009a:50), while Casual games generally refer to smaller games that prioritize matching sets, finding hidden objects, solving puzzles, and using mimetic interfaces. Casual gamers, in terms of their time spent playing, investment in play, and even play styles, can be - like Core gamers - extremely dedicated players (Begy and Consalvo 2011). While Casual games are not new (e.g. Solitaire and Minesweeper are both Casual), the current rise of social and mobile games, such as FarmVille and Angry Birds, is part of the Casual Revolution documented by Jesper Juul.

There has been much fanfare about the "Casual Revolution" (Juul 2010; Kultima 2009). As reported by Kultima, Casual games are a $\$ 2.25$ billion per year industry that is growing at a rate of $20 \%$ per year. Over 200 million people play Casual games each month over the internet (2009:58). The growth of Casual games is credited to their appeal to new gamers (i.e. those who have not played digital games before) as well as their ability to attract lapsed gamers (i.e. those who have played digital games in the past, but have not played recently). While Casual games are often treated as being diametrically opposed to Core games, Juul explains that what really separates the two is that Casual games are flexible toward being played in different ways, while Core games are inflexible, demanding that players be amenable to meeting the games' demands (2010:53). As Juul notes, the same game can be Core or Casual depending on how it is played. While Core games demand players' undivided attention for long periods of time (for example, requiring a player be online for hours at a time when raiding with their 
guild in World of Warcraft), Casual games are amenable to being picked up and played as a secondary activity (Kultima 2009). They can be played at the office while on a break, on mobile phones while waiting in line or on the bus, at home while socializing with friends, etc. Casual games fit easily into the daily routines of a much wider range of people. Moreover, Casual games have few technological barriers to entry, they are easy and quick to download, can be played on most computers, phones and handheld devices, and are less expensive than Core games (or altogether free). Challenging to play, and requiring large time commitments on behalf of the player, it is argued that Core games appeal to a much smaller market, historically comprised of young males (Kline et al. 2003). Accordingly, it is not surprising the Casual Revolution is fuelling most of the upheavals in the larger game industry.

\subsection{Core and the Second Spirit of Capitalism}

The struggles of the console industry can be located in terms of Core production models that are premised on the Second Spirit of Capitalism. They conflict with the new models of Casual sectors, whose structure and operation embodies the New Spirit of Capitalism. We can thus re-frame the Core vs Casual debate in terms of Industrial vs Projective Cités and their associated modes of production.

The Second Spirit of Capitalism began in the 1930's -1960's as family led

businesses slowly gave way to corporations that were run by managers and owned by stockholders. ${ }^{92}$ The Second Spirit emphasized the job security provided by economies of

92 Each Spirit promises a liberation from the critiques of the last Spirit. The Protestant Ethic promised a liberation from traditionalism - individuals could chose their job, social conditions, and practices of consumption through the capitalist workplace. The Second Spirit promised a liberation from capitalism, valuing the welfare state and job stability, and offering a respite from the forms of oppression. 
scale, rationalization, and long term planning. However, starting in the 1960's, this Spirit was heavily critiqued, especially in terms of the managerial problems associated with corporations' giant size. In these large firms, the negative side effects of bureaucracy multiplied: workers argued that they had little autonomy, and the emphasis on rationalization and efficiency - while upholding a meritocracy - left no space for creativity. Accordingly, these instrumentalized Taylorist workplaces turned workers into interchangeable and easily replaceable cogs in a machine.

These artistic critiques (i.e. focused on authenticity, creativity, and disenchantment with rationalized workspaces) induced a transformation in the operation and mechanisms of capitalism: "either through a direct response to critique aiming to appease it by acknowledging its validity; or by attempts at circumvention and transformation, in order to elude it without having answered it" (Boltanski and Chiapello 2007:167). These critiques were answered and incorporated into the New Spirit of Capitalism, which promised more worker autonomy by rejecting bureaucratic hierarchies and replacing them with lean companies that are organized in networks or by projects. Idealized New Spirit companies, by nature, are flexible and innovative. Because of the constant rate of technological change, companies and workers need to be flexible, adapt, and "be able to 'ride' all 'waves"' (Boltanski and Chiapello 2007:71). The internal flexibility of firms is based on transformations in the organization of work and the techniques employed (e.g. multitasking, self control, worker autonomy etc.) and the

characterized by early firms. In other words, the Second Spirit rejected the Domestic Cité values that underlay the First Spirit of Capitalism, and valued meritocracy, rationality, and professional management over the nepotism and personal judgements that characterized the Domestic Cité. In contrast, the New Spirit of Capitalism offers an escape from the massification and standardization of Second Spirit workplaces. 
external flexibility of Lean Projective firms that emphasize just-in time production, continual improvements in quality, and lots of outsourcing and contracting (Boltanski and Chiapello 2007:218).

The New Spirit of Capitalism emphasizes individual mobility and initiative. Each small team is run by a visionary leader, rather than waiting for orders to be passed down through the hierarchy, and networks of production are created by slim cores of stable personnel surrounded by contractors and allies. Workers are "malleable in terms of employments (casual jobs, temping, self-employed workers) working hours, or the duration of work (part-timers, variable hours)" (Boltanski and Chiapello 2007:218). As we will see in the next few chapters, many of these New Spirit of Capitalism traits are present in the console industry (e.g outsourcing, flexible work hours, etc.) yet artistic critiques from the 1960s remain: dissatisfaction with hierarchical management and other problems - such as the lack of autonomy - that are bound up with the unwieldy size of publishing, manufacturing, and development firms. This critique parallels the instrumentalization of play detailed in Chapter Four, showing how the work of those who create play spaces is increasingly instrumentalized as well. This rationalization of the game industry directly parallels similar movements in other culture industries, such as film and television. In fact, much of the academic writing on game industry economies takes a critical theory approach, drawing parallels between the film industry, book publishing, television industry and game development (see D. Williams 2002; Kline et al. 2003; Nieborg 2011). 


\subsection{The Rationalization of Console Game Development Processes}

In the console industry the quests for profits predominate. Production is streamlined and rationalized. The creative processes - the free play- of developers is greatly curtailed in order to maintain shrinking profit margins. Developers are the creative force behind games - conceptualizing, designing, and coding our spaces of play. But in the larger chain of console development they are a small cog in a rationalized production cycle, occupying a subservient position in contrast to publishers and manufacturers and bargaining from an undesirable negotiating position.

Three key players constitute console game development: development studios, publishers, and console manufacturers. For the sake of brevity, the roles of each of these players can be summarized as follows:

- Development studios create games;

- Publishers finance and promote the games to console manufacturers and retail distributors; and,

- Console manufacturers develop the hardware platforms on which games are played.

These three players are not on equal footing and there is considerable jockeying for position. Paradoxically, while developers are responsible for conceptualizing, designing, and programming the game, they occupy a position of little influence compared to both publishers and manufacturers.

The digital game production life cycle can be simplified into a five-stage process (see below), from development, to publishing, to manufacturing, to distribution, to retail. ${ }^{93}$ In the following paragraphs, I will describe each step in turn.

93 This figure is adapted from Williams (2002). Kerr's stages are slightly different: design, pre-production, production, publishing, distribution and retail. 
Illustration 6.1: Five stages of the digital game industry

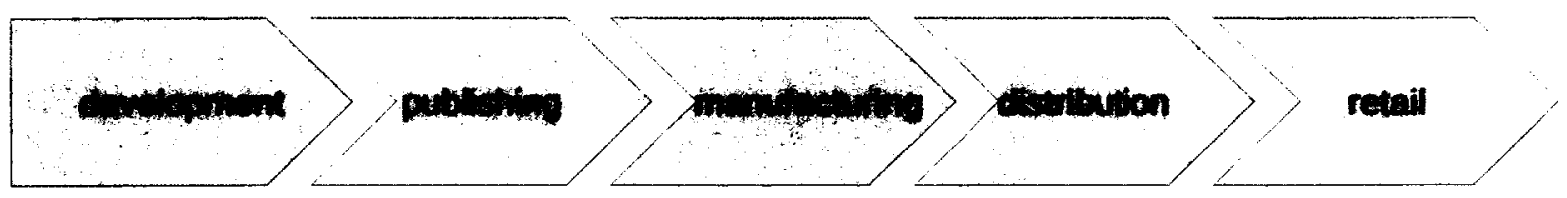

In the development stage, games are conceived, created, programmed, and tested. This stage can be broken up further into six smaller stages: the design stage, the prototype stage, pre-production, production, testing and quality assurance (QA), and finally after shipping the game, the final support stage. Generally, the development stage is carried out entirely within the development studio. Kerr provides an illustration, below, that breaks down the individual stages for console and PC games, using a 24 month long project to approximately show how long each stage took and how many development studio employees were involved at each level in 2006.

Illustration 6.2: Production stages and increases in staff on a 24-month project

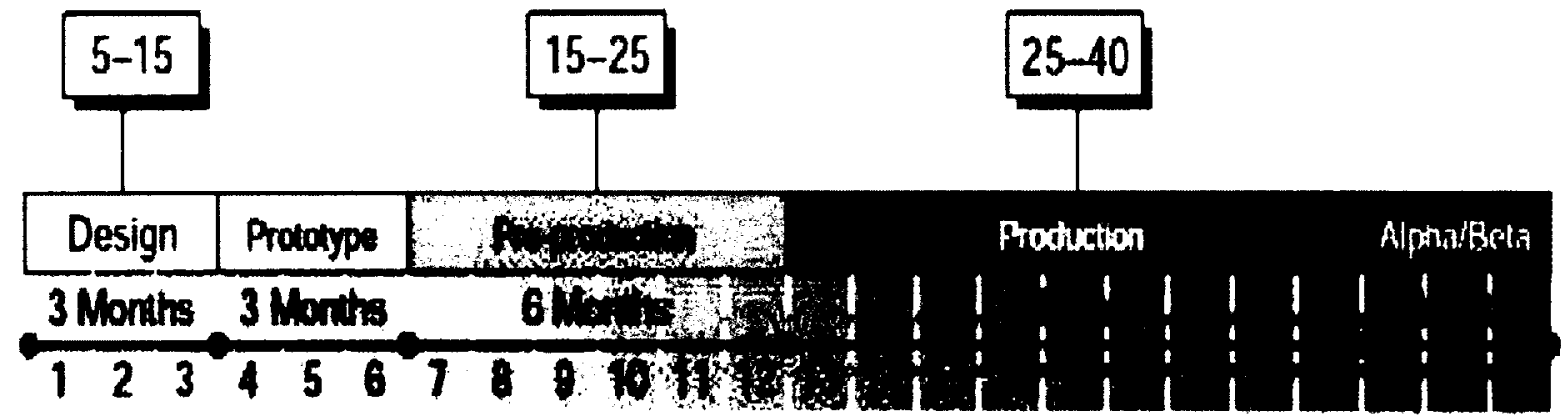

Source: (Kerr 2006:55)

Publishers take over the business side of games, ostensibly leaving development studios free to focus on the creative side of games. Traditionally, the publisher's role 
began once the game had been developed, tested and delivered. The publisher is also responsible marketing the game launch. However, shrinking profit margins in the console sector have compelled publishers to involve themselves throughout the development cycle, carrying out early market research and focus testing and suggesting changes to the game to attract a larger potential market. ${ }^{94}$ Publishers are also responsible for setting schedules and deadlines, ensuring the completed game is shipped at an advantageous time - typically in time for holiday shopping.

Publishers are also financially responsible for manufacturing the physical game. In the console industry, manufacturing game disks and cartridges is the exclusive domain of console manufacturers who must be contracted by the publisher. ${ }^{95}$ Publishers often take on the role of distributors, distributing not only the titles they produce but also the titles of competitors (D. Williams 2002). Distributors ${ }^{96}$ are responsible for the physical storage and delivery of the game product. They are also responsible for the sales effort, ensuring that the game reaches store shelves.

The retail stage is the only stage to avoid publishers' vertical integration. Retailers are the direct link to potential consumers and thus wield considerable power as they determine the visibility of the product for the mass market. The relationship between publishers and retailers is often contested and is the result of negotiations between these actors at the local, national and multinational scale (Johns 2006:171). Publishers compete

94 Although most developers would argue that their best work is done without interference from larger corporate structures (D. Williams 2002:46), there is a trend towards vertical integration as publishers and manufacturers in the console sector acquire development studios, looking to assert more control over the production process and thus resuscitate profits

95 This policy was instituted by Nintendo after the industry crash of the ' 80 's, as it allowed them to control the quality and quantity of the games produced for their system as well as effectively prohibit games made by other manufacturers.

96 In the early to mid 1990's distributors started to establish exclusive contracts with major retail chains. 
for limited shelf space. Those games that reach the shelves face a high turn-over rate and within a matter of weeks are replaced by newer games (Kücklich 2005). Accordingly, in big box stores, only games that have 'hit' potential are displayed. Retailers charge publishers additional market development funds to create posters, in-store displays, and other advertising. Publishers share the risk of the product's failure, promising that if the game sells poorly and copies remain on the shelf, the publisher will bear some of the store's discounting cost (which is then passed along to developers). While digital distribution means that publishers and developers may avoid the costs of the manufacturing, distribution, and retail stages, retailers maintain considerable power over the console sector. Games may be distributed digitally but game hardware, such as consoles and accessories, cannot. Accordingly, publishers are caught between developers wanting to focus on digital distribution and retailers who demand a continual supply of games as part of the agreement to stock game hardware. ${ }^{97}$

Illustration 6.3, below, breaks down the distribution of profits from a typical $\$ 60$ game. ${ }^{98}$ Approximately $20 \%$ goes to the manufacturer, $10 \%$ goes to the distributor (often the publisher), $30 \%$ to the retailer, and the remaining $40 \%$ is divided between the publisher and the developer.

97 This directly impacts the consumer. Although digital copies of a game are made much more cheaply than their physical counterparts, publishers have a vested interested in selling physical and digital games at the same price. If digital copies were sold more cheaply, retail software sales would decline precipitously and revolting retailers would no longer carry hardware and consoles, the latter of which already is sold below cost and is not very profitable even for retailers.

98 Input data was amalgamated from both Kerr (2006:66) and Johns (2006:165), and aligns with more recent numbers from Electronic Entertainment Design and Research (EEDAR) (Electronic Entertainment Design and Research 2011). 


\section{Illustration 6.3: Profit distributions for a $\$ 60$ game}

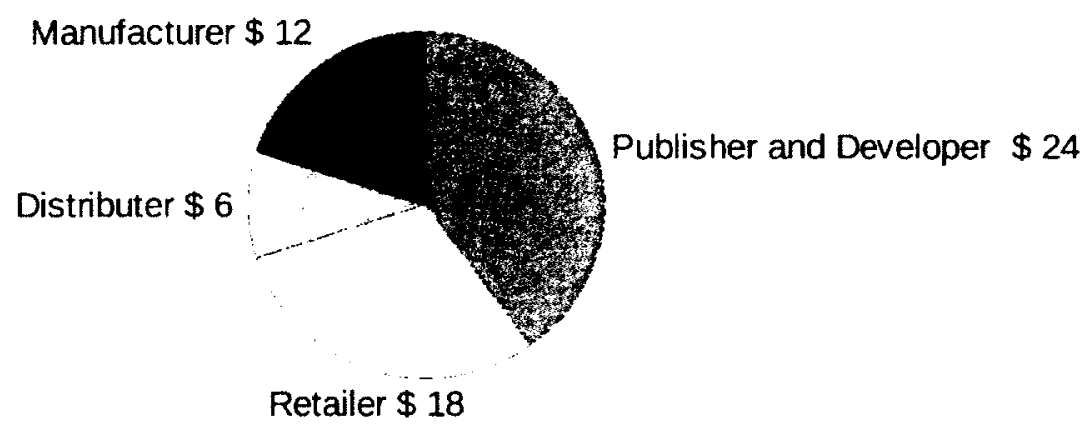

Negotiations of how profits are to be divided become a point of tension between business orientated publishers and the more creatively minded developers. These negotiations escalate as the Development stage becomes more more complex. Historically, console publishing and development was a high margin business, but these margins have succumbed to rising development costs and times. The essential production model of 2011 still remains the same as that detailed by Kerr's 2006 illustration (6.2) above, but schedules and team sizes have greatly increased. Production for retail console games can easily last more than 36 months in contrast to Kerr's average of 18 to 24 months, while teams sizes peak at over 200 developers, a far cry from the teams of 12 to 40 people that Kerr described (Kerr 2006:55; Gouglas et al. 2010).

The expanding scope and scale of development projects is directly related to the increasing technological complexity of each console generation - in this case, from the 6th to 7 th generation consoles. Not surprisingly, development costs have greatly inflated from the $\$ 3$-10 million estimated by Kerr (2006:26). Development budgets in the $\$ 20$ $\$ 25$ million range are now considered mid-size and have difficulty competing with more 
extravagantly funded projects (Graft 2011a). As the cost of development increases along with the cost of marketing - which matches and surpasses development budgets - the margin to be shared between publishers and developers steadily decreases. Thus publishers are pressured to vertically integrate and acquire development studios as a way to excise wider margins. The creates a trend towards conglomeration wherein publishing is concentrated in the hands of a few large corporations (e.g. Activision, EA, $2 \mathrm{~K}$, and Ubisoft), and the number of independent developers for consoles is decreasing (D. Williams 2002). While the game industry is often idealized as space of creating play, more often the 'creators' are at the mercy of corporate interests, particular those of manufacturers and publishers. This lies at the heart of the alienation and disenchantment faced by many game developers.

\subsubsection{Console Manufacturers}

The economic models behind game development greatly influence the form of digital games, but surprisingly little is written on the subject. Reports detailing the economic and organizational structure of game development share a common thread: the realization that "almost nothing" is known about the industry itself (D. Williams 2002; Tschang 2005; Kerr 2006; Cohendet and L. Simon 2007; Johns 2006). The little academic research there is focuses on manufacturers and the "console wars" wherein each new console ${ }^{99}$ attempts to outdo its predecessors in terms of advances in processing power. ${ }^{100}$ (Schilling 2003; Mateos-Garcia et al. 2010; Shankar and Bayus 2003; D. Williams 2002; Clements and

99 The 8th generation is predicted to begin with the release of the Wii's successor in 2012/2013. 100The Wii shirks this trend, focusing on motion-sensing technology rather than improvements to graphics or processing power. 
Ohashi 2004; Afuah and Grimaldi 2005).

The high price point for consoles means that most players can only afford one console, and thus there is only room for a small number of firms. As summarized by both Shilling and Williams (Schilling 2003; D. Williams 2002), there is an disincentive for console manufacturers to work together as they are all fighting over the same potential user base: One firm's gain in network effects is likely another firm's loss, so the drive to acquire those consumers first is all-consuming. Console manufacturers are oligopolisitic and endorse closed technological systems, thus expressing a strong structuring effect on the game development process. They erect a number of barriers to protect their market share and prevent the entry of competitors, going to great lengths to control the flow and quality of content onto their system and to ensure that non-licensed software from nonaccredited developers will not work on their platforms (Kerr 2006:57). It follows that this structural antipathy should and has promoted cartel-like behaviour that negatively impacts developers (D. Williams 2002:44).

In order to attract the largest install base, console manufacturers take a 'razor blades' approach, selling the console at a loss while hoping to profit from software sales. ${ }^{101}$ The more games sold, the more the manufacturer can reduce the cost of the platform. Each platform price drop drives more platform and game sales. While market share is dependent on the sale of consoles, console sales are directly related to the number

101For example, when the Playstation 3 (PS3) launched in 2006, the 20GB unit was sold for $\$ 499$ while estimated production costs were $\$ 800$. This meant that Sony was selling at an estimated loss of over $\$ 300$ for every 20GB unit (Graft 2011c). In the previous generation, Microsoft had to sell eight or nine games for every Xbox in order to break even. As described by Kline et al., the more Xbox units Microsoft sold, the more money it lost: "Some internal sources suggest the company will lose eight hundred million dollars over the Xbox's first four years, and two billion over eight years before it can even think of turning a profit" (2003:175). 
of high-quality games available. In the console war, manufacturers recruit developers as expendable foot soldiers. Platform holders compete to achieve a dominant design status by enlisting coalitions of developers to provide games that then attract users to the console, increasing the console's install base with the aim of accruing greater market shares and thus suffocating competitors. Once they have gained the largest market share, leading manufacturers often abuse their power with unreasonable contract offers for developers, or poor quality products for consumers, colloquially termed as "shovelware". Platform manufacturers rely on software sales to recoup hardware losses via licensing and manufacturing agreements with developers and publishers. For example, outside developers who create games for Sony, Nintendo, and Microsoft pay a per-copy licensing fee that ranges from $\$ 5-\$ 8$ per unit. ${ }^{102}$ Manufacturing fees to create the disks at the manufacturer's plant add additional costs of $\$ 1-3$ per disk. This means that console manufactures profit from each game manufactured, regardless of whether these games are ultimately sold. Developers must also purchase development kits from console manufacturers that vary in price from $\$ 10,000$ to $\$ 20,000$ each. ${ }^{103}$ Smaller debugging kits can be purchased at a fraction of the cost (e.g. $\$ 1,000$ ), but these kits do not offer the same level of functionality. Both kits are issued only to registered developers who are pre-approved by console manufacturers. Manufacturers also impose stringent quality controls referred to as Technical Certification Requirements (TCRs or 'certification' for short). These extra fees and barriers work to offset manufacturer's production and marketing costs, consequently keeping the per unit price of each game high while 102Current licensing fees are difficult to find. Kerr (2006) states that licencing fees for Nintendo were generally about $\$ 7-\$ 10$ a game.

103Jason Della Rocca verified that this is the current price range, however the price declines as the console ages and nears the end of the generation. 
restricting the number of developers who can create console games.

By controlling the game manufacturing process, console manufacturers increase their control. This enables them to design anti-piracy measures, ensuring that only games with these measures are playable on their consoles. It also allows for quality control, monitoring the industry outputs, and importantly, allows them to claim a percentage of revenue for each game sold. This gatekeeping ability is a source of direct power over publishers, as they only use approved publishers who have gone through a formal vetting process, thus creating an inner circle of preferred suppliers.

\subsubsection{Publishers}

Publishers occupy a place of power as the connecting node between manufacturers and developers. Because there only three manufacturers, they give publishers the authorization to sell games for their platform. This creates strong links between publishers and manufacturers, and between publishers and development studios (Cadin and Guerin 2006:253). Publishers - not players - take the role of 'buyers' by selectively providing funds for game development projects, signalling to developers what to make. They are "'governors' of the value chain in that they have the financial resources to develop games and, critically, to market them; these resource needs far exceed those available internally to development companies" (Readman and Grantham 2006:257). Publishers pay developers advances to cover development costs, these advances are released only if developers meet pre-established progress milestones on time. In exchange for assuming the financial risk, publishers typically acquire the intellectual 
property rights for the game as well as advantageous terms for future sequels.

Publishers are the target of much game developer critique, largely because of the constraints they put on developer creativity and autonomy. Console game development is a formulaic, hits driven business (D. Williams 2002; Kerr 2006; Kline et al. 2003). Only 3 percent of digital games make a profit (Kerr 2006:45). Thus, hits must cover the production costs of a large number of failures. This provides an incentive to stick to tested themes and mechanics. Publishers introduce similarity and predictability into this process, commissioning formulaic games that fall into particular generic categories. As Kline et al. summarize, this risk aversion creates a pressure towards mainstream content; "a relatively restricted repertoire of games promoted by well-connected publishing houses, and towards marginalizing or asphyxiating the projects of developers outside this circle" (2003:179). Additionally, in order to reduce marketing costs, which can match or exceed development costs, games are often tied with established franchises or IP that consumers are already familiar with (e.g. basing games on toys such as LEGO or books such as Harry Potter), and thus further limiting creative exploration or the development of new game genres. Publishers' bargaining position with manufacturers is often less than ideal, as evidenced by the following quote from a publisher:

The bulk of the risk of financing projects is carried by us, the publishers. They don't manufacture the game until we have paid them and their only other costs are their own internal costs. The actual material cost of producing a game is probably less than $€ 2$, so if they are charging us anywhere between $€ 7$ and $€ 10$, that is really where they are making their money. (as quoted in Johns 2006:163)

In these cases, rather than occupying a position of power, publishers are caught between manufacturers and developers. 


\subsubsection{Developers}

Developers provide the game code in exchange for a fixed fee and/or fixed percentage of sales revenue. The illustration below highlights the different stages of development as game code goes from developers, to manufacturers and publishers, to distributors and retailers before finally reaching the consumer. Rather than the flexible, adaptable production networks epitomized by the New Spirit of Capitalism, console game development is largely hierarchical, with each element (i.e. developer, manufacturer, publisher) highly interdependent on the 'chain' of command.

Paradoxically, rather than being the central node of the game industry network, developers occupy a peripheral space in comparison to manufacturers and publishers. They are charged with the creative development of the game code, but the rest of the production network is overseen and controlled by publishers. 


\section{Illustration 6.4: Game development's actors and interconnections}
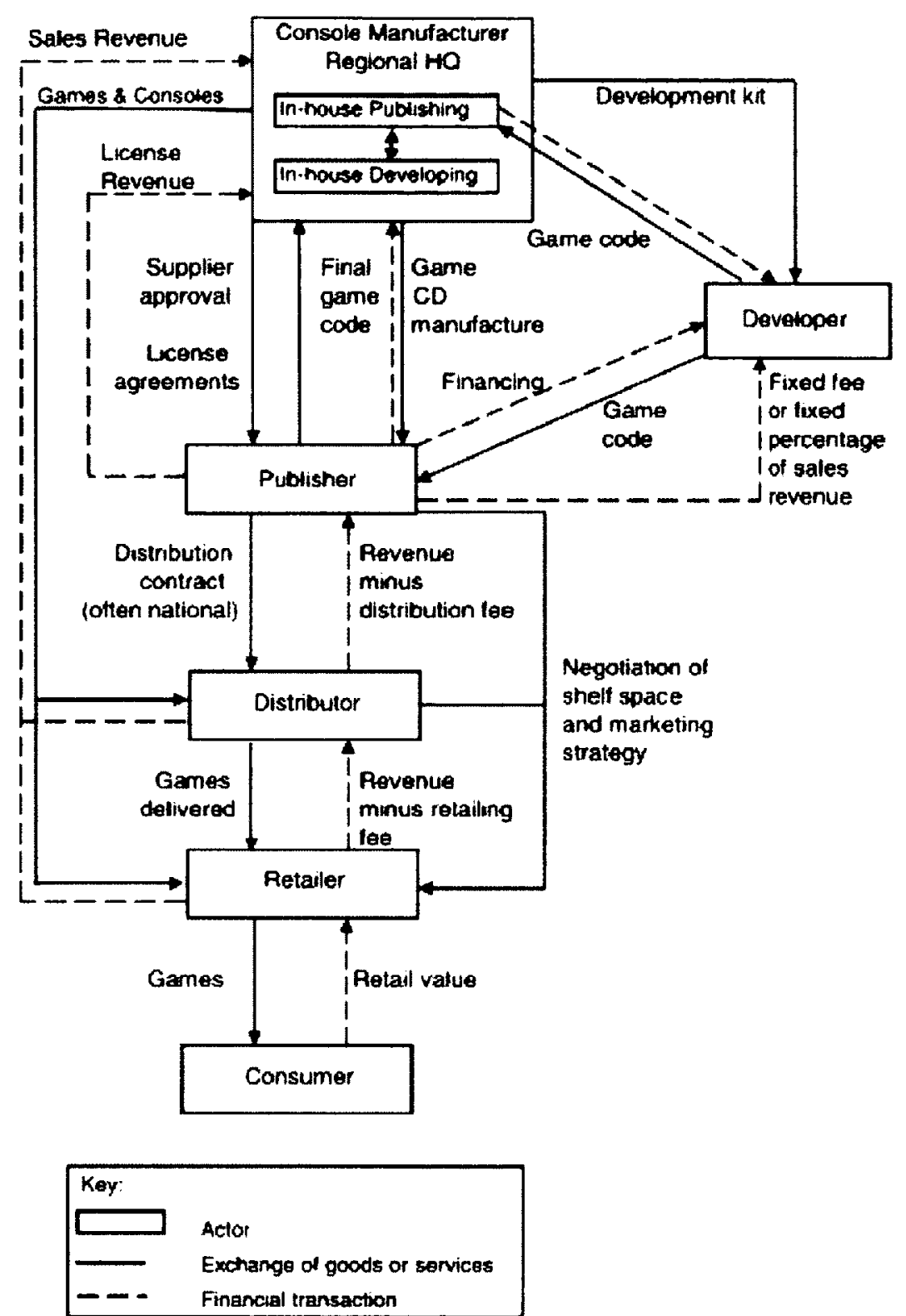

Source: (Johns 2006:164)

Larger companies, especially publicly traded console manufacturers and publishers, are accountable to shareholders and thus favour consistent quarterly reports 
over taking risks. Commonly, publishers only become involved in game development after large portions of the game are already developed. In the event that the developer retains the IP rights for a successful new game, publishers may attempt to purchase the entire development studio in order to control the franchise. O'Donnell argues that once a start-up is able to prove itself and produce a successful game, it "is acquired and then strapped down with milking the value out of those resources" (2008:164). Developers who chafe under these larger corporate hierarchies leave to start new companies and begin the cycle over again - consequently pushing the industry in new directions. This offloading of risk onto independent developers serves to create a perpetual start-up cycle within the industry. The risk of developing new IP or innovative products is most often borne by smaller independent companies who collapse if the project proves unsuccessful. Developers are certainly constrained by this production process, which subordinates their creativity to numerous other desires (e.g. economic efficiency, creating a mass market hit, publishing in time for the holiday shopping season, etc.). They are also exploited by the console manufacturers and the console life cycle.

\subsection{The Console Life Cycle}

Developers negotiate with publishers and manufacturers from a disenfranchised position. The competition for limited funding contributes to the lack of developer organization and bargaining power. The negotiation power of individual developers depends on the reputation and history of the development studio, the reputation of individuals employed by the studio, and importantly, the timing. Developers' negotiating power fluctuates 
according to where consoles are in their individual life spans (Johns 2006; Schilling 2003; D. Williams 2002). At the start of a console cycle, developers have more negotiating power. As the manufacturer gains market share in the later stages of the life cycle, developer negotiating power drops, as depicted by the illustration, below.

\section{Illustration 6.5: The variable bargaining power of game developers}

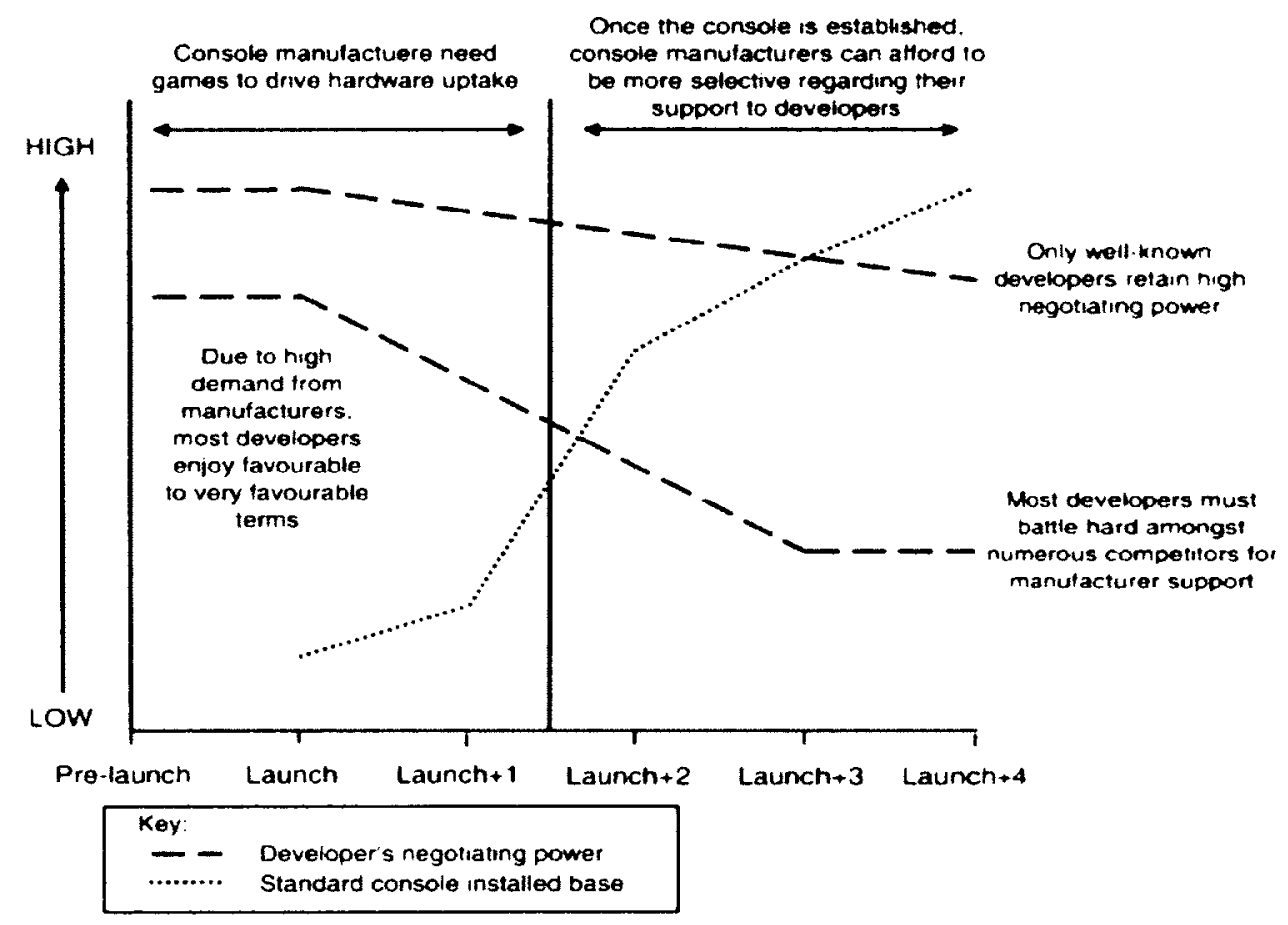

Source: (Johns 2006:170)

A more detailed analysis of these fluctuations is outlined by game designer Daniel Cook, former employee of Microsoft Game Studios and co-founder of the game development company, Spry Fox. He separates a platform's lifespan into four periods. ${ }^{104}$ From a

104Instead of focusing on console manufacturers, Cook broadens his analysis to platforms more generally, showing how patterns from the console industry are replicated by newer platforms, like Facebook. 
developer perspective, different risks and rewards occur at different points in the platform life cycle. For example, when new consoles are released developers are courted with favourable terms. But as the console gathers market share, developer freedoms are eroded and the platform holders become more abusive (Cook 2011a).

\subsubsection{Emerging Phase}

Developers must be attracted to a platform early in its life cycle, as having a large number of games for the birth of a new system is instrumental to the system's success (Schilling 2003). This is a difficult task for new console manufacturers, as developers do not want to waste resources on a system that has few users or is unlikely to survive the years' long game development process. It is difficult to predict whether a nascent system will ultimately succeed (D. Williams 2002). Accordingly, console manufacturers offer development studios sweetheart deals early in a console's life cycle.

\subsubsection{Engage Phase}

During the "engage phase" the console has succeeded in attracting initial customers, but requires outside help in scaling up production and providing new content for the growing population of users. Thus, manufacturers engage with outside developers. As Cook explains, manufacturers are welcoming to third party developers as they are relatively inexpensive to contract. It is these developers that assume the risk in trying out new things and testing what is possible with the system. Console manufactures are particularly interested in enabling and then promoting the success of specific developers as this 
success - especially their posted profits - attracts more developers to the console.

However, the console manufacture strives for total control over "killer apps", as these $20 \%$ of games drive $80 \%$ of traffic. ${ }^{105}$

\subsubsection{Extract Phase}

During the "extract phase" console manufacturers have achieved enough market share to ensure their continued survival. Enough players have purchased the console that the manufacturer now has distribution power: they control access to new customers. If game developers want access to these customers they must acquiesce to the console manufacturer's terms. Because many developers are competing to reach the console's users, the console manufacturer is not concerned about alienating a few developers, especially because developers are comparatively fragmented and lack the bargaining power that would come from being organized. At this point, the terms do not overtly favour developers and their percentage of revenues continually decreases. Conversely, regulations and constraints increase. ${ }^{106}$

\subsubsection{Decline Phase}

The "decline phase" is characterized by developers leaving for greener pastures. Most often these pastures are competing consoles at earlier, more welcoming, phases of the life

105For example, Microsoft acquired Halo developer Bungie in 2000. When Bungie separated from Microsoft in 2007 Microsoft retained the hit franchise's IP rights. Nintendo is an even more extreme case as most games released for the Wii are first party games.

106It is a common pattern for the largest and most successful firms to abuse their market power in negotiating with developers. This abuse of power and constraint of developers was directly responsible for Nintendo losing market dominance to Sega in the early 1990's. It was repeated in the mid 90's by Sony surpassing Nintendo (D. Williams 2002:45). 
cycle. But, as we will see in Chapter Nine, increasingly, developers leave console production for Casual games. As developers begin to drift away, fewer games are released and the console's market share diminishes as gamers move to other systems.

\subsection{Conclusion}

A majority of console game projects are economically doomed - "only $4 \%$ of the games that go into production, and $20 \%$ of those which are eventually launched, manage to cover their costs" (Mateos-Garcia et al. 2010:7). This means out of every twenty-five games that start production, only one will be profitable. As Mateos-Garcia et al. argue, the console industry has become a dominant design that is no longer sustainable (2010). The escalation in budgets has not been accompanied by market growth, partly due to the lack of continued financial investment in console games, and the increasing number of cheaper venues for games. As the scope and sophistication of console games increase, delays, budget over-runs, and quality issues increase in frequency (Mateos-Garcia et al. 2010:7). Surviving console studios must continually work harder just to maintain their financial position. With lowering profit margins, tensions between developers, publishers, and console manufacturers increase. There are ways to streamline development even further, making it more cost effective and efficient, such as outsourcing; however, as console development chains become more streamlined, game development itself becomes much less playful. And games themselves may follow.

Like Grimes and Feenberg in the earlier chapter on the instrumentalization of play, Casey O'Donnell links the increasing instrumentalization of play with moves 
towards professionalization of sport and leisure and the emphasis on 'official' rules to regulate and commodify what was previously uncommodified free play (2008) ${ }^{107}$ For example, in baseball, the playing fields, the rule-sets, and the equipment used - right down to the grass on the field and the weight and composition of the bats - have all been standardized and regulated in order to ensure a 'fair competition' that all spectators can understand and follow (and thus would want to pay to watch). A homogenous experience results, reducing the potential for the spontaneous negotiation of rules that is part of more informal game play.

In O'Donnell's case, it is not just the games themselves that are being professionalized, but also the professionalization of development. As this chapter has evidenced, console games are not playfully thrown together in a garage but are produced within sociotechnical assemblages that are increasingly large and globally distributed. The complexity of these production processes, by necessity, require intense levels of coordination and collaboration that must be enabled by growing levels of precision - hence necessitating increased surveillance to facilitate a seamless collaboration across technical and spatial divides. Of course, the size of this social-technical assemblage means that commercial profit-driven organizations (i.e. manufacturers and publishers) become part of the system, providing the funds needed to bankroll production and the managerial expertise required to direct sophisticated collaboration. But commercial profit-driven organizations are playing a different game than game development employees. As iterated by O'Donnell:

It is not simply the systematization and regimentation of game/sport that

107 For example, baseball rules, stadiums, and equipment have all been standardized 
causes the loss of game-like innocence. The issue is larger than that. Simply bringing money into the whirlwind does not automatically cannibalize your game. The difficulty is that money brings those interested in playing other kinds of games into connection with what many had hoped might stay a game. It is the incorporation of a drive towards institutionalization that changes the game. (O’Donnell 2008:70)

The goal of profit-driven organizations (e.g. publishers, retailers, distributors) is not to create a 'fun' game, or a desirable place of play. Their goal is to create a profitable enterprise. Their goals sometimes align with those of game developers (e.g. 'fun' and innovative games like Little Big Planet can be profitable), but often they do not (e.g. the emphasis on low-risk formulaic sequels). In terms of the development and production of games, the time needed to play with and learn technical systems, and the time needed to experiment with different iterations of a game is deemed wasteful and inefficient. And as the potential profits of the game industry increase as the market of potential players increases due to emerging game sectors, we can only expect the commodification and instrumentalization of play to increase.

This chapter used the New Spirit of Capitalism as a framework to more broadly situate and explain the struggles of the console industry, reframing Core vs Casual in terms of industrial vs networked modes of capitalism. This chapter sets the stage for Chapter Nine and the Casual Revolution, showing how economic pressures on production processes greatly constrain how games are made, but also which games get made and who can make them. But economics is only part of the console picture. The next chapter addresses developer culture and its relation to instrumental play. While the economy greatly influences game development in terms of the increasing instrumentalization of play, developers are not passive observers, but rather exhibit agency of their own. 


\section{Chapter 7. Maintaining the Machine: Game developers and Core culture}

This chapter is a continuation of the last, situating console developers within Core culture. While developers resist and critique the production hierarchies and limits on creativity that constrain Core development, they also work to protect Core norms and values, thus paradoxically upholding the economic systems that create these constraints. Accordingly, any understanding of the game industry cannot ignore the role of developers themselves. This chapter uses the instrumentalization of play to first tie together Chapter Four to console development. It then broadens the discussion to show how the instrumentalized play of developers contributes to and reinforces exploitative working conditions. Lastly, it shows how developer communities of practice reinforce cultural homogeneity and resist innovations (e.g. new platforms, technologies, or game genres) that do not align with their pre-existing Core values.

The game industry discourses that reproduce the symbolic order of the New Spirit of Capitalism also work to reify and maintain the shared identities of developers. My primary data, particularly online sources such as Gamasutra that cater to console and PC developers, reveal an important conflict. These sources readily admit to undesirable working conditions and long periods of unpaid overtime. This was also a common topic in my interviews with developers. As we saw in the previous chapter, developers voice additional concerns about lack of creativity and the excessive constraints imposed by manufacturers and publishers. However, at the very same time, Core developers are incredibly vocal in their opposition to, and denigration of, other game sectors - especially social games. Accordingly, the motivation for this chapter was to further understand how 
developers form a sense of culture and shared identity premised around Core ideals.

\subsection{Developers and Instrumental Play}

Any discussion of instrumental play and the blurring boundaries between work and play would not be complete without the work of Casey O'Donnell $(2008,2009)$. O'Donnell's three year ethnography of game studios in North America and outsourcing companies in India traces how the fun and play commonly associated with creating games has become characterized by instrumental play. A key aspect of O'Donnell's argument is that instrumental play has not only come to dominate games, it permeates the game industry as well. Just as playing games is a increasingly rationalized practice, so to is the creation of games.

The development of video games, and the software industry as a whole, is commonly idealized as a playful endeavour characterized by freedom, autonomy, and joy. Graduates of computer science, engineering, digital arts, and information technology fiercely compete to land jobs at popular studios, attracted by promises of Foosball tables, free candy, pop and food, and comfy couches for napping. Of course, the opportunity to create a beloved game often seems more like play than work. Like power gamers, employees demonstrate an ambivalence about what constitutes legitimate work and - at least at first - they happily 'play' much longer than 9-5, 5 days a week. In periods of "crunch" they work throughout the night, fuelled by caffeinated beverages and catching a few moments of rest on the couch. O'Donnell suggests that we can fruitfully conceptualize game development as a game itself, especially since many game 
developers describe their own work in terms of a game. But this game, at least in terms of how it is currently configured, is one for power gamers.

The language of developers' work/play is the language of games. It is a common language shared by all employees: the engineers who have come up through computer science, electrical engineers, physicists, artists, designers, writers, and managers. Each of these groups speak a highly specialized language of their own ,and thus communication between these different groups would be difficult, if not impossible, without a common touchstone: the games they all play. ${ }^{108}$ The game talk that unites these diverse disciplines situates employees within a realm of play rather than work. ${ }^{109}$ References to games, game mechanics, play styles, and genres become a means of understanding and communicating about the underlying systems and structures of the games they are working to create. Game talk is a working manual that instrumental work/play relies upon, especially in cases where a set of clear standards, rules and codes do not exist. Beyond providing a game design blueprint to riff off of and improve upon, game talk, especially that propagated by game reviews and postmortems, solidifies what counts as a "good" game, what is an innovative mechanic, and which endeavours are a "step in the right direction", etc. $^{110}$

1080'Donnell notes that, while providing a common sense of purpose, a moral code, and social system, the language of games can be used to exclude as well as include. Those who do not grow up playing games cannot speak the language of power-ups, button combos, cut-scenes and permadeath. They are strangers in a strange land, and as such, have a difficult time grokking the system. Consequently, the Indian developers followed by O'Donnell - because they grew up in a third world country without access to digital games - were unable to break into the inner echelons of the industry and develop the insider technical knowledge and network connections needed to bootstrap their own studios.

109This means that, like the gamification detailed in Chapter Three, the magic circle of play is overlaid on everyday work practices.

110 As indicated by a number of my interviews, game talk becomes an essential tool to speak not only to other developers, but to publishers as well. Studios were careful not to pitch new games as innovative or groundbreaking, as publishers responded better to known quantities (where revenues were easier to project). Accordingly, developers felt external pressure to copy and adapt existing games and pitch them 
Like power gamers, game company employees demonstrate the underlying drive and dedication towards the efficiency and instrumental orientation first discussed by Taylor (2003). They are committed to understanding the underlying game systems and structures, and developing their technical skills and proficiency, demonstrating each of the five key properties of reflexivity, boundedness, rule-governedness, precision, and playfulness that I described in Chapter Four. In this sense, "instrumental work/play is the process/drive/desire to determine how to get the most out of the systems required to get the job done" (O’Donnell 2009:2.1). Each discipline within a development team relies upon highly specialized technical systems, working within them to create the complexly woven and increasingly intricate code of the game: "Instrumental gaming is essential to game development. In many cases it is impossible for artists, engineers, or designers to know what precisely they can manage to create without causing the underlying hardware to buckle under the pressure they put on it" (2008:64). Much of their work/play can be framed in terms of grokking these technical systems (e.g. in-house software tools, middleware, console requirements), reflexively determining just what each system can do, finding their boundaries, and playfully pushing up against the rules of the system, and sometimes modding and remaking these rules in order to create novel and cutting-edge play experiences. Part of this play includes attempting things that should not work and finding the play between the rules of the system in an iterative process of exploration and play. With games, players must intuitively feel their way through the system, and at the outset are only provided with brief tutorials and terse user manuals. Game development is

in recognizable terms such as: "it's like Bioshock but multiplayer and social", or "the game is like Call of Duty meets Dead Space" or "it's like Mass Effect in its narrative system, but set in an World of Warcraft environment". 
a similar undertaking, wherein employees are given initial specifications and design visions, but frequently find themselves having to feel their way towards the goal of a finished product, reflexively learning about the underlying systems along the way.

Like games themselves, the work/play of game development is increasingly instrumentalized. Echoing Taylor's discussion of power gamers, workers are encouraged to become pathologically dedicated and work with superhuman determination (Costikyan 2009; Dyer-Witheford and de Peuter 2006). There is less opportunity for iterative free play and exploration (paidia), and more emphasis on efficiency, reaching the goals of milestones, certifications, and shipping dates in a timely manner and under budget. While some development studios emphasize the paidiac aspects of development, encouraging innovative modes of play or refusing to ship a game until it is ready ${ }^{111}$, the overall organization of the game development industry, especially in terms of the socioeconomics of development, promotes instrumental play and, in particular, the increased commodification of games and the surveillance of both players and employees. Using Taylor's terminology from Chapter Four, game development itself has become a "soft coercive system" (2006a). As with games, employees freely choose to engage with the system, but their actions are undeniably constrained and oriented towards certain directions. Their acceptance of the daily grind and gruelling years-long production cycle is something that comes from the bottom-up and is not just imposed from the top-down. Much like the surveillance mods described by Taylor in Chapter Four, while the organization of the system encourages certain forms of rationalized play, players (in this

111 Indie 'garage' developers with low overhead often characterize this experimental aspect of work/play, but I would argue that Valve, with its early experiment with digital distribution methods (Steam), crossplatform play and willingness to push back shipping dates (Portal 2) is a AAA example of allowing developers space for paidiac play. 
case, developers) take an active role in shaping, encouraging, and propagating these Core play styles. Game sectors that generally affiliate with Core games and their associated values and culture are console, PC, MMOs, and indie. While not all developers in these sectors make Core games (e.g. see family-focused console titles such as WiiPlay, or Mario Party), they generally all come from a Core gamer background (Mateos-Garcia et al. 2010; Cohendet and L. Simon 2007; O’Donnell 2008).

\subsection{Core Culture and the Working Conditions of the Power Gamer}

While alternative markets and platforms exist (as we will see in the following chapters), the console space dominates the imagination of developers. This predominance is due to the allure of AAA budgets and cutting edge technologies, but it is also rooted in the very exclusivity of the network, "It is a social indicator of having breached the intranetworks of the video game industry. It is a status marker" (O'Donnell 2008:55). Gaining access to the console game development network marks the aspiring developer as privileged and elite, a status that particularly resonates with hardcore gamer/developers. In this sense, part of the fun and play of game development work is because the closed nature of the network promotes exclusivity, and the belief that "If you do not know these things already, then likely you are not one of us" (O'Donnell 2008:286). ${ }^{112}$

O'Donnell highlights the role of developer culture in maintaining the status quo

112 In contrast to the exclusivity of Core, emerging sectors were populated initially by Casual interlopers and outsiders to the game industry proper, people with traditional web developer backgrounds, who "cut their teeth on figuring out landing pages, conversion funnels, A/B testing, and so on" (M. Su 2010). These web designers applied techniques from advertising and web design to attract massive audiences to relatively simple games. While Core developers are moving into these emerging sectors, they continually come into conflict with the value systems embedded in these new sectors, especially in the case of using metrics and quantitative measures to drive design rather than creative intuition (Brathwaite 2011b; S. Johnson 2010). 
for console development, a topic that is also addressed by Mateos-Garcia et al. (2010). In O'Donnell's words, there is something about games that enables and encourages game developers to push harder and longer than they would otherwise. There is a conflation between work and play that "encourages them to forge new connections and think creatively. These new modes of work practice are simultaneously crucial, yet capable of being pushed to too far, dissolving into destructive work practices" (O'Donnell 2008:19). These destructive work practices have come to epitomize the Core industry.

Part of the allure of working for a development studio is rooted in the atmosphere: unregulated work hours, loose dress codes, free food, on-site fitness facilities, and lavish interior design (Dyer-Witheford and de Peuter 2006:604). ${ }^{113}$ The largest proportion of game industry employees is between their late teens and thirties. Accordingly, the youthful stamina of these employees sets a norm of overwork and late nights, in an "enforced youthfulness" that characterizes many creative-industry workplaces (DyerWitheford and de Peuter 2006:610). Kline et al. (2003) argue that by making work more like play, employers erase the division between the two, ensuring that employees will almost never leave the office. The cool ambiance of the studio and the renegade reputation of star developers mask the fact that the working conditions are themselves far from ideal.

The length of the working day varies widely depending on the company, where the development team is in a game's life cycle, and the worker's role in the project. The work conditions of developers are commonly characterized by extended periods of

113This description applies to the software industry more generally (e.g. the offices of Google, or even local Ottawa offices), and not just to the game industry. 
unpaid overtime, "crunch", in order to meet production deadlines. The IGDA Quality of Life Whitepaper (International Game Developers Association 2004) surveyed developers and reported that:

1) Crunch time is omnipresent. During crunch, $30 \%$ of respondents worked $55-65$ hours a week, while over $35 \%$ of respondents worked 65 to 80 hours a week. A further $13 \%$ exceeded 80 hours a week;

2) Overtime for nearly half of all employees is uncompensated (46.8\%); and,

3) Spouses are likely to respond that "You work too much..." (61.5\%); "You are always stressed out." (43.5\%); "You don't make enough money." (35.6\%).

Even outside of crunch periods, the workday of the average developer is punishing: nearly $60 \%$ of developers work 46 hours or more on a typical (non-crunch) week, while nearly $20 \%$ more report working over 55 hours a week. Reports of working more than 100-hour weeks are not unheard of. The most common forms of compensation are time off at the end of a project, profit-sharing, and royalties. Few companies pay overtime in actual cash.

Quality of Life issues in the game industry are a relatively well-examined academic topic, especially in relation to the media attention attracted by the thenanonymous blog posts of "EA Spouse" that detailed EA working conditions (see DyerWitheford and de Peuter 2006; Kline et al. 2003). The aforementioned IGDA Whitepaper and other related initiatives were developed in direct response to the crisis (Hyman 2007). However, similar tales of workplace exploitation surface on a continual basis (e.g "THQ Spouse" and "Rockstar Spouse") (Costikyan 2009; Remo 2010a; Acton 2011). This is partly due to the fact that managers and developers often see crunch as a normal part of doing business (Dyer-Witheford and de Peuter 2006) and no-crunch policies are 
extremely rare. ${ }^{114}$

While most accounts of EA Spouse argue that game developers represent the most exploited post-industrial employees (Dyer-Witheford and de Peuter 2006; Kline et al. 2005), O'Donnell charges that the overviews of the EA Spouse phenomenon lack nuance, focusing on bad management or corporate greed on the part of EA rather than examining the broader contexts of the game industry. A key element that is lost in EA Spouse articles is that explanations for worker exploitation are inadequate, especially since many fans and aspiring developers work the same hours for free (see Humphreys 2005; Postigo 2003; Kücklich 2005). This moves beyond discussions of coercion and co-option in the workforce and co-option to something deeper that is rooted in the compulsions and underlying values and norms that are bundled with work/play.

Mateos-Garcia et al. (2010) echo O'Donnell's assessment, asserting that developers' willingness to work unpaid overtime (a mirror form of the playbour of users generating game content) is a manifestation of intrinsic motivations rather than exploitation or monetary rewards. Simply put, developers may not embrace alternative modes of game production because they love the form of games they create, and they cannot envision alternative methods of doing things. This finding parallels Taylor's assertion that players have a role in creating soft coercive systems (2006a). MateosGarcia et al. use the concept of "path dependence" to further explain developer's resistance to change. A term used by historians (David 1985) and Science and Technology Studies (STS) (Jackson et al. 2007), path dependence looks at the history of

114For example, in the IGDA Quality of Life Survey, only $2.4 \%$ of respondents reported having no crunch at all because their company mandated 40 hour work weeks. 
objects and processes to explain organizational and market inertia without the need to assume that actor's behaviours (in this case developers) are irrational. As put by economic historian Paul David, "it is sometimes not possible to uncover the logic (or illogic) of the work around us except by understanding how it got that way" (1985:332). Once established, systems - even inefficient ones such as the QWERTY keyboard that David chronicled - tend to continue in particular directions, making reversals or wholesale leaps to alternative approaches costly, difficult, and in some cases impossible. In this case of Mateos-Garcia et al. (2010), developers themselves exhibit a cultural path dependency, and an unwillingness to leave the Core games and the power gaming habits they have developed over years and decades behind - even if they contribute to their unfavourable working conditions.

Mateos-Garcia et al. counteract the marginality of developers assumed by other researchers and evidence the role that developers have in contributing to the emergence of a dominant design (i.e. Core console games). They re-assert the agency of individual developers that is marginalized or lost altogether in purely economic accounts of how the video game industry operates, and contribute to broader path-dependence literatures by providing a third alternative, beyond agents who are unable to deviate from a historically determined trajectory versus agents who exercise agency and work to break away from it. In this third situation actors "exercise their agency to sustain an existing, inefficient path with historically dependent features because they derive non-economical benefits from doing so" (Mateos-Garcia et al. 2010:2). This third approach explains why 'rational' developers avoid other development platforms in favour of Core console development, 
even though it makes economic sense for them to embrace the alternatives. Accordingly, Mateos-Garcia et al. emphasize the power of developer culture and communities of practice.

\subsection{Core Communities of Practice}

"Communities of Practice" is a term developed by cognitive anthropologist, Jean Lave, and computer scientist, Etienne Wenger (Lave and Wenger 1991). It broadly refers to people who share a common interest and a desire to learn from, and contribute to, a community - in this case game development. Newcomers learn through practice and participation (situated learning), establishing shared norms and collaborative relations. The enterprise of making games together, and a general love of games, become a shared repertoire that unite the members of a community of practice together (see Wenger, McDermott, and Snyder 2002). Mateos-Garcia et al. (2010) studied the organizational, management and recruitment practices of seven UK development studios to understand developers' resistance to new (Casual) platforms, specifically the Wii. They found that these practices resulted in a cultural homogeneity in both workforce and management positions, creating tightly-knit communities of practice. These communities favour the incremental innovation detailed by Ernkvist and Ström (2008) that ultimately obstruct the exploration of alternative dominant designs and presents a danger for their future economic success.

The culture of the development workforce shapes what games are made, irrespective of whether certain games would be more profitable economically. In the 
console industry, the workforce is heavily skewed towards the demographics of Core gamers. Developer communities of practice, despite being comprised of specialists from many diverse disciplines, are culturally homogeneous. Mateos-Garcia et al. emphasize the dangers of a homogenous developer community:

Cultural homogeneity in the workforce leads to the 'introspective tendencies' identified in the data. Studios tend to make 'the games they want to make', with only marginal attention - or even respect for - wider audiences. This impacts on the dominant definition of quality - something that is realistic, visually striking, technically sophisticated and 'cool', and will be respected by other peers and the specialist games press. It also results in the adoption of usability testing practices that assume a high level of user proficiency. (Mateos-Garcia et al. 2010:15)

A homogeneous creative community may work to increase collaboration among members and increase efficiency, but it also precludes access to new perspectives and sources of inspiration. ${ }^{115}$ This means that there is a smaller potential for creative diversity and inspiration, creating rigidities and creative stagnation as developers fall prey to a path dependence and willingly create more of the same, exploiting existing genres and mechanics rather than exploring the potentials for new ones. For example, the pool of influences that developers draw from when exercising creativity is narrow, narrated by nods to science fiction and fantasy and structured by references to other games and their mechanics.

Similar to Mateos-Gracia et al. (2010), Aoyama and Izushi (2008), and Ernkvist and Ström (2006), both Tschang (2005) and Cohendet and Simon (2007) focus on communities of practice. However, they take micro-level approaches in examining how individual developers within a studio work together and argue that, while developers may

115This incidentally explains why emerging sectors, such as gamification, social and mobile games, were initially populated by game industry outsiders, such as web designers and management professionals. 
exhibit some homogeneity, the varied specializations and backgrounds of development teams contributes to innovation. However, the separate disciplines are still aligned by their love of Core games which becomes a common bond that ties these diverse occupations together and contributes to a larger community of practice.

The recruiting and workplace organization of development studios serves to reinforce cultural homogeneity. When new developers are recruited they come directly from an 'amateur' talent pool of hardcore gamers (for example, play-testing games is one way for aspiring developers to get a foot in the door of development studios). Like Mateos-Garcia et al. and O'Donnell, Cohendet and Simon acknowledge that the video game industry generally hires from a pool of Core users, who speak the same lingua franca of games that developers do. This provides clear benefits: " Sharing a common language and common references, they would most likely agree on the generic features differentiating a good game from a bad one. This common cognitive platform allows them to work collectively as designers and also to promote their passion for video games"(Cohendet and L. Simon 2007:594). While the common Core culture allows employees to talk to each other from across vast disciplinary divides, it also makes it difficult to establish a dialogue with the "elusive" Casual gamer. ${ }^{116}$

Homogeneity is encouraged through self-selection - those passionate about games overlook the quality of life issues endemic in game studios, while those who do not identify as strongly with the dominant Core mentality will seek employment somewhere with more favourable working conditions and job security. On the management side,

116In my experience, this Casual gamer is depicted as "your mom" - a middle-aged female who is diametrically opposed to the twenty-something year old males that dominate most game studios. 

developers want to hire employees that are passionate about their work, who know games, and who will fit in with the staff. Experienced developers are recruited upwards through the management hierarchy, thus carrying developer culture and the associated ideas of what makes a good game upwards along with them.

This naturally results in the hiring of individuals who are demographically and culturally similar to the rest of the development studio employees. This selects for predominantly young male developers. Female employees are rare, due to a number of factors. As Dyer-Witheford and de Peuter argue (2006), developers hire candidates with previous experience in the game industry or a passion for games. Since games have predominately been marketed to young males, the result is an industry built around games made by males, for males. In comparison, potential female employees may lack gaming experience. The dearth of female representation is also linked to the gendered nature of 'feeder' disciplines, such as computer science, where female enrolment is traditionally low. Finally, the lack of females is due to a general risk aversion in the industry and the related lack of funding for developers who want to create games that appeal to a crossgender audience.

While new employees have the potential to inject fresh lifeblood into the system, they assimilate into the preexisting developer culture rapidly, thus locking games studios into an "'introspective' and self-referential trajectory of improvement along conventional dimensions of quality - graphical polish and 'cool features' in triple A games - instead of encouraging them to explore new genres, or target emerging platforms" (Mateos-Garcia et al. 2010:16). In other words, potential employees are willing converts to the 
established console path rather than trail-blazing missionaries.

Communities of practice can produce complacency and overconfidence in

established formats, genres, and game mechanics. While communities of practice lead to familiarity and trust between members (and in the case of workplaces, this leads to efficiency and cooperation), this discourages the formation of new networks with 'outsiders' and experimentation:

The combination of complacency and familiarity thus locks individuals and firms in the existing networks of their communities. As this goes on, the networks start to show their age. Unable to bring together information from different sources and perceive new opportunities, the networks become less flexible and less innovative than they used to be. (Aoyama and Izushi 2008:8)

Simply put, console developers affiliate around their love of Core games. This is what draws them into the industry. It becomes a binding community of practice. This means that developers are resistant to alternative dominant designs that do not seem to cater to Core values. While catering to a much larger non-Core (i.e. Casual) market makes economic sense and may reduce many of the economic tensions in the industry, developers would prefer to create games they are passionate about; games that they would play themselves. ${ }^{117}$ Accordingly, Core developers often do not understand the allure of other alternative non-Core formats (e.g. Casual consoles such as the Wii, or social games on Facebook).

117As Kerr notes, designers and developers base their assumptions about users on their own tastes and interests (2006:6). On the publisher side, most market research is on existing gamers: young males. And playtesting and de-bugging is carried out by this same population of passionate players. Accordingly, most feedback on games is from the same select population, reinforcing the notion that the Core market that publishers and developers should aim for is still males, age 13 to 25 (Kerr 2006:98). This influences the rate of innovation across all levels of the industry, from deciding which games to fund, to marketing, to designing the underlying consoles. 


\subsection{Conclusion}

Console development is rarely kind to developers. To reiterate Chapter Six, in the console industry the quests for profits predominate. Production is streamlined and rationalized. The creative processes - the free play - of developers is greatly curtailed in order to maintain shrinking profit margins. Development studios are the creative force behind games - conceptualizing, designing, and coding our spaces of play. But in the larger chain of the console industry they are a small cog in a rationalized production cycle, occupying a subservient position in contrast to publishers and manufacturers, and bargaining from an undesirable negotiating position. Yet, despite this, developers themselves are resistant to change. Part of this resistance is rooted in the finely tuned technological skills, aptitudes, and specializations these "power developers" have developed over time. Moving to something different would mean leaving the familiar, learning new systems, and starting anew. Resistance to change is also rooted in developer culture and a shared passion for Core games. Their passion for the work means that developers are often willing to overlook working conditions that are less than ideal, profit models that benefit those that sell and market games rather than those that create them, and contracts that stifle creativity in favour of less risky clones or sequels of games that are already proven to be profitable.

At some point developers inevitably reach a breaking point. As the statistics above show, most developers burn out quickly and abandon the industry within five to ten years. And the continued economic viability of the console market - at least in a form predicated upon physical retail sales - is in doubt. Increasingly, game developers argue that the 
economics of the mainstream industry stifle all creativity. As summarized by Chris Hecker $^{118}$, detailing his own shift from mainstream to indie development ${ }^{119}$ :

The obvious thing is in a triple-A title, for the most part, people are very risk averse because that's someone's fifty million dollars. People don't tend to want to lose that. Because people want a return on investment in the mainstream industry it has a direct impact on design. Because you're not given a very long leash. For doing really creative things you need to be able to fail and meander aimlessly for a while, while you're finding it right?

In order to maintain the essence of Core culture, console developers like Hecker have become critics of the hierarchical console development processes (just as protestors took the role in May 1968). Operating in a highly constrained and commodified Culture Industry, they ask for more autonomy and creative ownership over their work. The changing revenue models and alternative sectors detailed in the next chapter show how Capital has adapted in response, answering many of their economic critiques and promising some escape from publisher and manufacturer hierarchies. Accordingly, the following chapters ask these questions:

- What does the future of game development look like? How is it it different from traditional models of Core console development?

- Do these new emerging models offer a respite from the constraints and tensions characterized by console development?

- How do these emerging models work? How are they profitable?

- Do these models off more freedom, play, or power to developers?

- What do these new models of production mean for the shape of games and the experience of players? and,

- How is the instrumentalization of play enhanced by these emerging trends?

118Hecker goes on to criticize the indie community for being resistant to change. Even free from constraints that preclude experimentation, developers evidence a path trajectory - creating the same forms of games they played in their youth.

119Chris Hecker is a programmer who worked for both Microsoft and Maxis (where he worked with Will Wright on Spore). He was also Editor-at-large at Game Developer Magazine and speaks at numerous conferences, including the Game Developers Conference. He now creates indie games and is the cofounder of the Indie Game Jam. 
The next chapter will begin outlining the answers to these questions, illustrating the broadening landscape of game development. 


\section{Chapter 8. Blueprints for New Machines: Shifts in game development landscapes}

So far in this thesis, I have made the link between Spirits of Capitalism and the game industry, arguing that console development and Core culture are tied to the industrial, hierarchical models of production idealized by the Second Spirit of Capitalism. I have argued that the Instrumentalization of Play means that games are increasingly seen as rationalized tools, and that both playing games and making games increasingly focuses on goal-oriented actions and efficiency rather than free play and creativity. I have also alluded to the struggles of the console sector as it competes with new Casual sectors that are premised on the New Spirit of Capitalism and networked agile modes of existence. While the previous chapters outlined traditional modes of production and the constraints of console production, this chapter details how the industry is changing in response. By examining a range of game development sectors and focusing, in particular, on how Core sectors are adapting in response to emerging Casual sectors and digital distribution, this chapter begins to answer the questions posed at the end of the previous chapter. In this chapter, I outline the shifting landscape of game development from 2006 to 2011 , focusing on how Core sectors are changing to offer the creativity, adaptability, and flexibility denied by the Second Spirit of Capitalism. These changes are largely brought about by online connectivity and digital distribution.

This chapter heavily relies on the primary data from my online sources, listed in Appendix C. These sources provide vital information on what the game industry landscape looks like, how competing production models and economic models work, and, how team sizes, development schedules and budgets fluctuate according to game sector. 
Importantly, these sources also report on how established game sectors are attempting to stay alive by adapting innovations from emerging sectors. Those online sites that provide financial analysis of the industry as well as strategic advice about market trends, such as Industry Gamers, GamesIndustry.Biz, and GAMESBrief were essential tools in researching this chapter, as were Game Developer postmortems that provided details on teams sizes, development schedules, and budgets.

\subsection{Game Industry Sectors}

In 2006, Aphra Kerr classified the game industry into four sectors, each with its own unique revenue models and production processes (Kerr 2006). The two major sectors were console games and "standard" PC games, both of which used similar production methods and were distributed using traditional retail models (i.e. boxed product sold in stores). The key difference between these two sectors was that console game development involved an extra player - the hardware manufacturer - and unlike PC developers, console developers could not create or release console games without the approval of the manufacturer.

In a unique move for economic analyses of the game industry, which generally focus on the console industry and mention the PC sector only in passing, Kerr documented two emerging sectors: MMOs and mini-games. ${ }^{120}$ The latter category emphasized Casual gaming, including the games like Solitaire and Minesweeper that came pre-packaged with computers, and included early mobile and browser games that

120Indie games, a sector present from the birth of digital games such as Spacewar!, to Atari homebrew, to shareware and onwards, are rarely mentioned in economic analyses. However, they are an ever-present influence on the industry. 
were digitally distributed and supported via a number of different revenue models. Generally, "mini" games were dedicated to the Casual market (e.g. housewives, people playing games at work), while the Console, PC, and MMO sectors catered to the Core demographic (e.g. young males).

While traditional sectors (e.g. console and PC) were dependent upon physical game sales through brick and mortar stores, Kerr's emerging sectors experimented with relatively new payment models - such as charging a monthly subscription for MMO games. Mini-games exhibited the greatest variation in revenue streams. Casual game portals offered games on a subscription or pay-per-download basis, but others were supported by advertising revenue and were thus free-to-play (F2P). Mobile games were sold online and profits divided between the developer and the mobile company, while a few game channels were included as part of cable television products. Importantly, in contrast to Console Games \& Handhelds, PC Games, and MMOs, Mini-games were developed within short time spans and with smaller teams. The Mini-game model was nascent in 2006 but has grown exponentially, since then destabilizing the bastion of console development and initiating shifts throughout the entire games industry.

While the categories defined by Kerr still exist, the economic and distribution models of Consoles \& Handhelds, PCs, and MMOs have adapted. Entirely new economic and distribution models have led to a revitalization of Indie games and the growth of downloadable content and games for the console and PC industries. Mini-games have now expanded into a number of different sectors including: casual games, social games, and mobile games. On the periphery, gamification companies have latched onto the 
popularity of social and mobile games, using similar design techniques and distribution methods to make everyday life more playful. ${ }^{121}$

Illustration 8.1: The gaming landscape in 2011

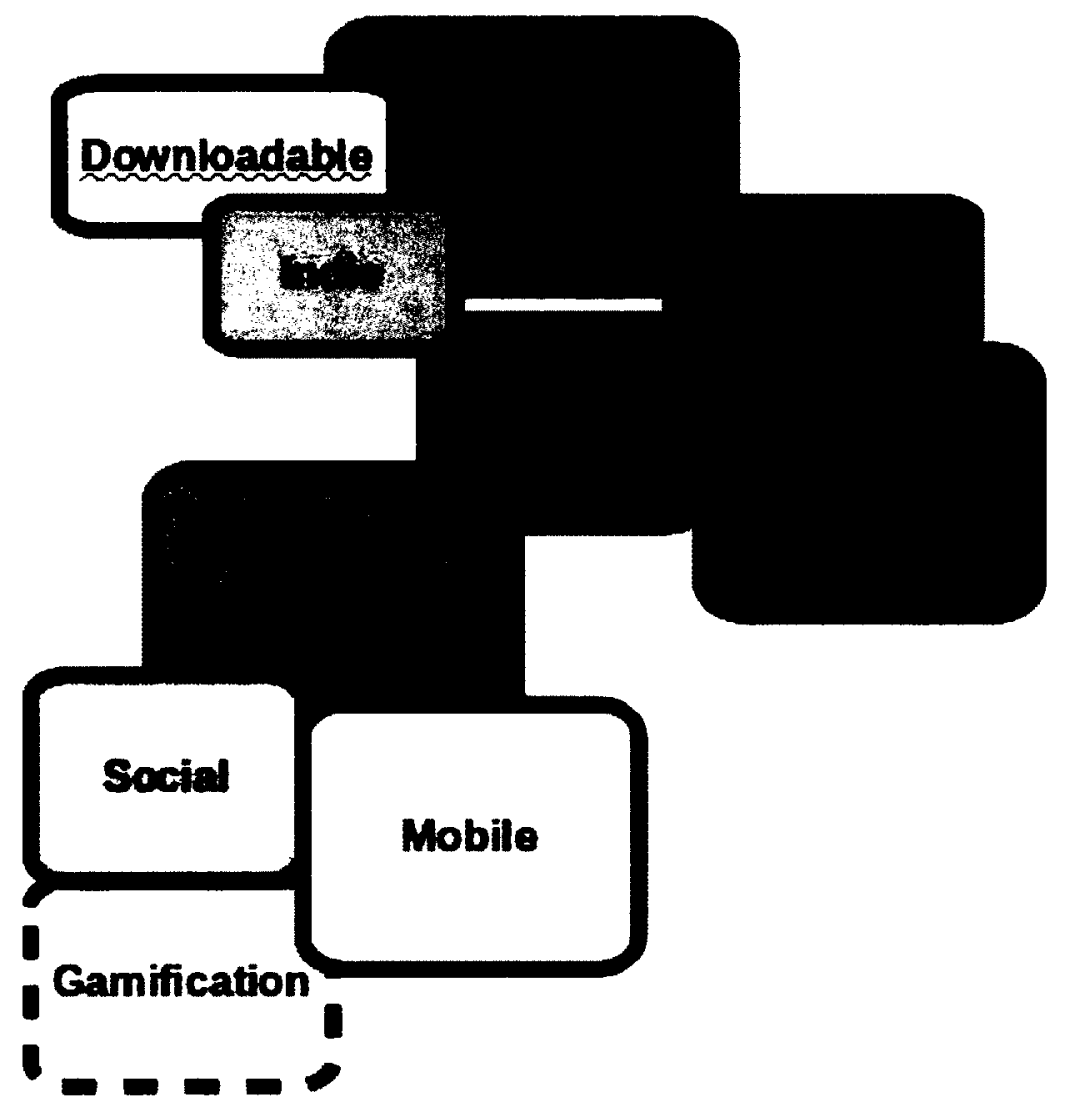

Table 8.1, below, provides a brief comparison of each of the different sectors. ${ }^{122}$ This table draws from a similar table by Kerr (2006:55-56). It has been updated to reflect numerous changes including growing team sizes, new revenue streams, and new sectors.

121This list of sectors is not exhaustive. Other sectors include art games (often lumped with indie games), serious games including games for health, workplace training and education, social change games, etc. I have written about them elsewhere (Whitson and Dormann 2011; Dormann, Whitson, and Biddle 2011). 122Gamification exists on the far periphery of game development. Accordingly, it is not included in the table. 
The table is organized in two sections, highlighting the divide between Core and Casual,

a central theme of this chapter.

\section{Table 8.1: Key Sectors of the digital games industry CORE SECTORS}

\begin{tabular}{|c|c|c|c|c|c|}
\hline & $\begin{array}{l}\text { Examples of } \\
\text { Platforms and } \\
\text { Games }\end{array}$ & $\begin{array}{l}\text { Market } \\
\text { Concentration } \\
\text { (Hardware \& } \\
\text { software) }\end{array}$ & Revenue Model & $\begin{array}{l}\text { Openness of } \\
\text { Hardware } \\
\text { System }\end{array}$ & $\begin{array}{l}\text { Software Production } \\
\text { Process }^{123}\end{array}$ \\
\hline $\begin{array}{l}\text { Sector } 1 \\
\text { Console } \\
\text { Games }\end{array}$ & $\begin{array}{l}\text { Console } \\
\text { Halo Reach on } \\
\text { the Xbox } 360 . \\
\text { Little Big } \\
\text { Planet on the } \\
\text { Playstation 3, } \\
\text { Super Mario } \\
\text { Galaxy on the } \\
\text { Nintendo Wii. }\end{array}$ & $\begin{array}{l}\text { Hardware } \\
\text { oligopoly } \\
\text { Sony, } \\
\text { Microsoft } \\
\text { Nintendo. } \\
\text { Limited } \\
\text { number of } \\
\text { approved } \\
\text { publishers and } \\
\text { developers. }\end{array}$ & $\begin{array}{l}\text { Hardware developed as a loss } \\
\text { leader and money made on sales } \\
\text { of software. } \\
\text { Game discs sold through shops. } \\
\text { Premium retail price. } \\
\text { Most games online and } \\
\text { multiplayer functionality. } \\
\text { Downloadable content (DLC) } \\
\text { sold online and in stores. }\end{array}$ & $\begin{array}{l}\text { Closed. } \\
\text { Proprietary } \\
\text { and non- } \\
\text { interoperable } \\
\text { hardware } \\
\text { systems. }\end{array}$ & $\begin{array}{l}\text { Games expensive to } \\
\text { develop ( } \$ \text { tens of millions } \\
\text { and upwards). } \\
\text { Varying follow-up service } \\
\text { costs that increase with } \\
\text { multiplayer functionality } \\
\text { and DLC. } \\
\text { Average length of dev. } 18 \\
\text {-48 months. } \\
\text { Average team size } 50 \text { to } \\
\text { over } 200 \text {. }\end{array}$ \\
\hline $1 \mathbf{b}$ & $\begin{array}{l}\text { Handheld } \\
\text { Scribblenauts } \\
\text { for Nintendo } \\
\text { DS, Patapon } \\
\text { for Sony PSP. }\end{array}$ & $\begin{array}{l}\text { Hardware } \\
\text { oligopoly } \\
\text { Sony and } \\
\text { Nintendo. } \\
\text { Limited } \\
\text { number of } \\
\text { approved } \\
\text { publishers and } \\
\text { developers. }\end{array}$ & $\begin{array}{l}\text { Hardware developed as a loss } \\
\text { leader and money made on sales } \\
\text { of software. } \\
\text { Game discs sold through shops. } \\
\text { Premium retail price. } \\
\text { Downloadable content (DLC) } \\
\text { sold online and in stores. }\end{array}$ & $\begin{array}{l}\text { Closed. } \\
\text { Proprietary } \\
\text { and non- } \\
\text { interoperable } \\
\text { hardware } \\
\text { systems. }\end{array}$ & $\begin{array}{l}\text { Games less expensive to } \\
\text { develop ( } \$ \text { hundreds of } \\
\text { thousands to single digit } \\
\text { millions). } \\
\text { Low service costs. } \\
\text { Average length of dev. } 12- \\
24 \text { months months } \\
\text { Average team size } 10-50 \text {. }\end{array}$ \\
\hline 1c & $\begin{array}{l}\text { Console } \\
\text { Downloadable } \\
\text { Castle } \\
\text { Crashers on } \\
\text { XBLA, Fat } \\
\text { Princess on the } \\
\text { PSN, Bit.Trip. } \\
\text { Beat on } \\
\text { WiiWare. }\end{array}$ & $\begin{array}{l}\text { Hardware } \\
\text { oligopoly } \\
\text { Sony, } \\
\text { Microsoft } \\
\text { Nintendo. } \\
\text { Numerous } \\
\text { publishers and } \\
\text { developers. }\end{array}$ & $\begin{array}{l}\text { Hardware developed as a loss } \\
\text { leader and money made on sales } \\
\text { of software. } \\
\text { Games downloaded online } \\
\text { through console. } \\
\text { DLC sold online. } \\
\text { Lower retail price than 1a. }\end{array}$ & $\begin{array}{l}\text { Closed. } \\
\text { Proprietary } \\
\text { and non- } \\
\text { interoperable } \\
\text { hardware } \\
\text { systems. }\end{array}$ & $\begin{array}{l}\text { Games less expensive to } \\
\text { develop ( } \$ \text { hundreds of } \\
\text { thousands to single digit } \\
\text { millions). } \\
\text { Low service costs. } \\
\text { Average length of dev. } 10 \\
-18 \text { months } \\
\text { Average team size } 5-20 \text {. }\end{array}$ \\
\hline $\begin{array}{l}\text { Sector } 2 \\
\text { Standard } \\
\text { PC Games }\end{array}$ & $\begin{array}{l}\text { Crysis, Team } \\
\text { Fortress 2, The } \\
\text { Witcher, Sid }\end{array}$ & $\begin{array}{l}\text { Numerous } \\
\text { hardware } \\
\text { manufacturers, }\end{array}$ & $\begin{array}{l}\text { Games sold through shops or } \\
\text { online distribution platforms. }\end{array}$ & $\begin{array}{l}\text { Common } \\
\text { standards, } \\
\text { non- }\end{array}$ & $\begin{array}{l}\text { Games less expensive to } \\
\text { develop than full console } \\
\text { games ( } \$ \text { tens of millions) }\end{array}$ \\
\hline
\end{tabular}

123Data sets for these averages are from interviews and postmortems published from January 2010 - May

\footnotetext{
2011. They are approximations.
} 


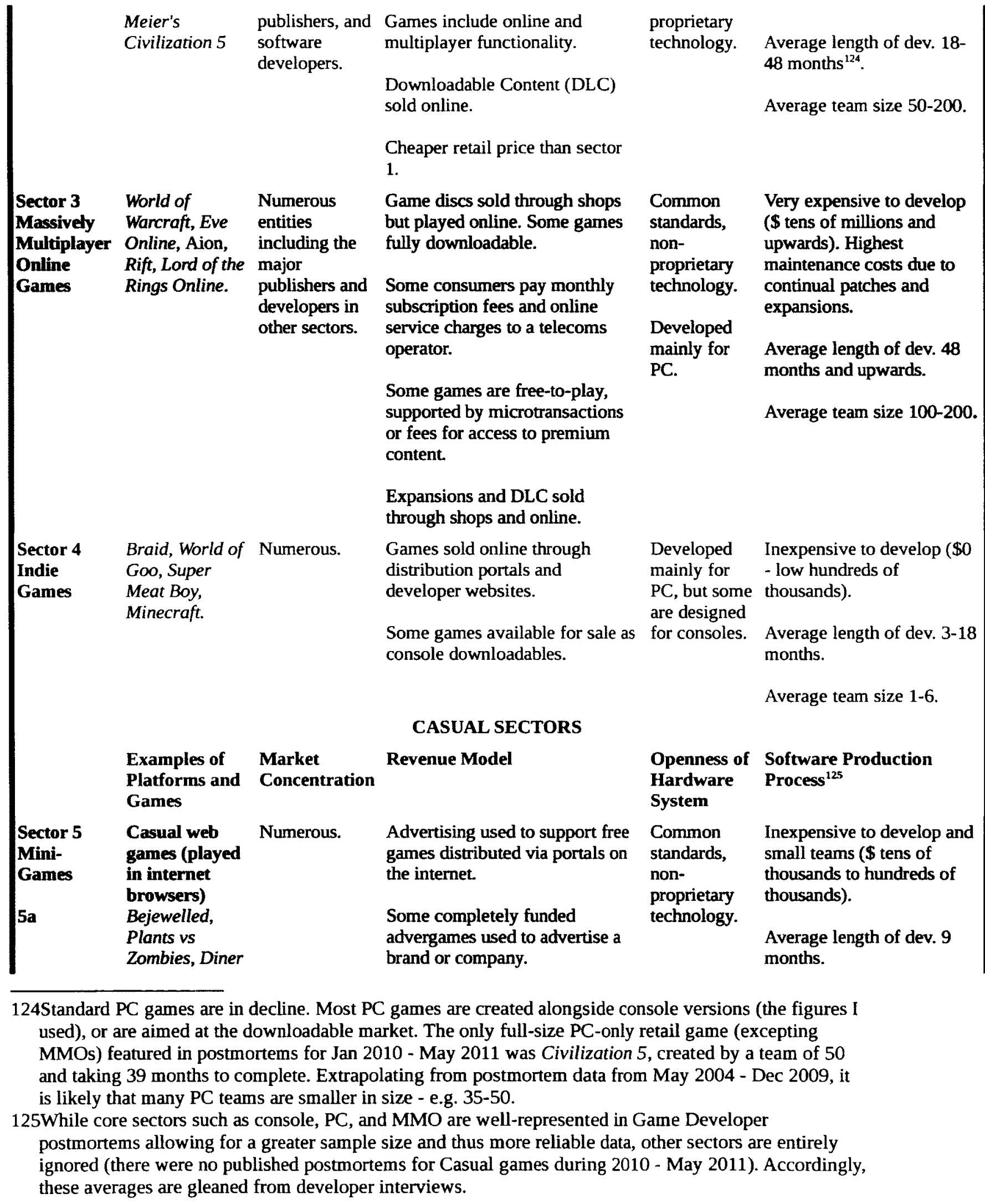


Dash.

Some games are free-to-play, supported by microtransactions or fees for access to premium content.

Also pay-per-play and monthly subscriptions.

$5 b$

Mobile Angry Birds, Cut the Rope, Rolando.

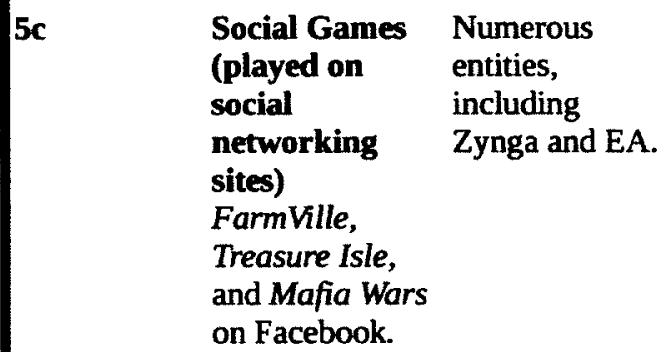

Numerous Games sold online through paydevelopers and per-download model. publishers.

For iOS (i.e. the iPhone and iPad), the Apple App Store is the only publisher.
Some games are free-to-play, supported by advertising, microtransactions or fees for access to premium content.

Revenue divided between developer and operator (e.g. Apple App Store, Amazon, etc.).

Games are free-to-play, supported by advertising and microtransactions.

Revenue divided between developer and operator (e.g Facebook).
A number of competing proprietary technologies.

A number of competing platforms and input devices.
Inexpensive to develop and small teams ( $\$$ tens of thousands to hundreds of thousands).

Average length of production 6 weeks-3 months, but continually expanding.

Inexpensive to develop and small teams ( $\$$ tens of thousands to hundreds of thousands). Long term service costs.

Average length of production 6 weeks-3 months, plus continual updates

The above table, while useful for untangling and differentiating the different sectors, is a snapshot in time. Team sizes and budgets for downloadable and social games exhibit continual growth, while the diversity of revenue models within each sector are increasing. As we will see in Chapter Nine, there is also a constant exchange of ideas, techniques, methods, and technologies between the sectors. Console and PC games are not disappearing, but their market growth has halted while online games and mobile games show continued revenue growth, as exhibited by Illustration 8.2, below. 


\section{Illustration 8.2: Global digital games sector revenue (\$B)}

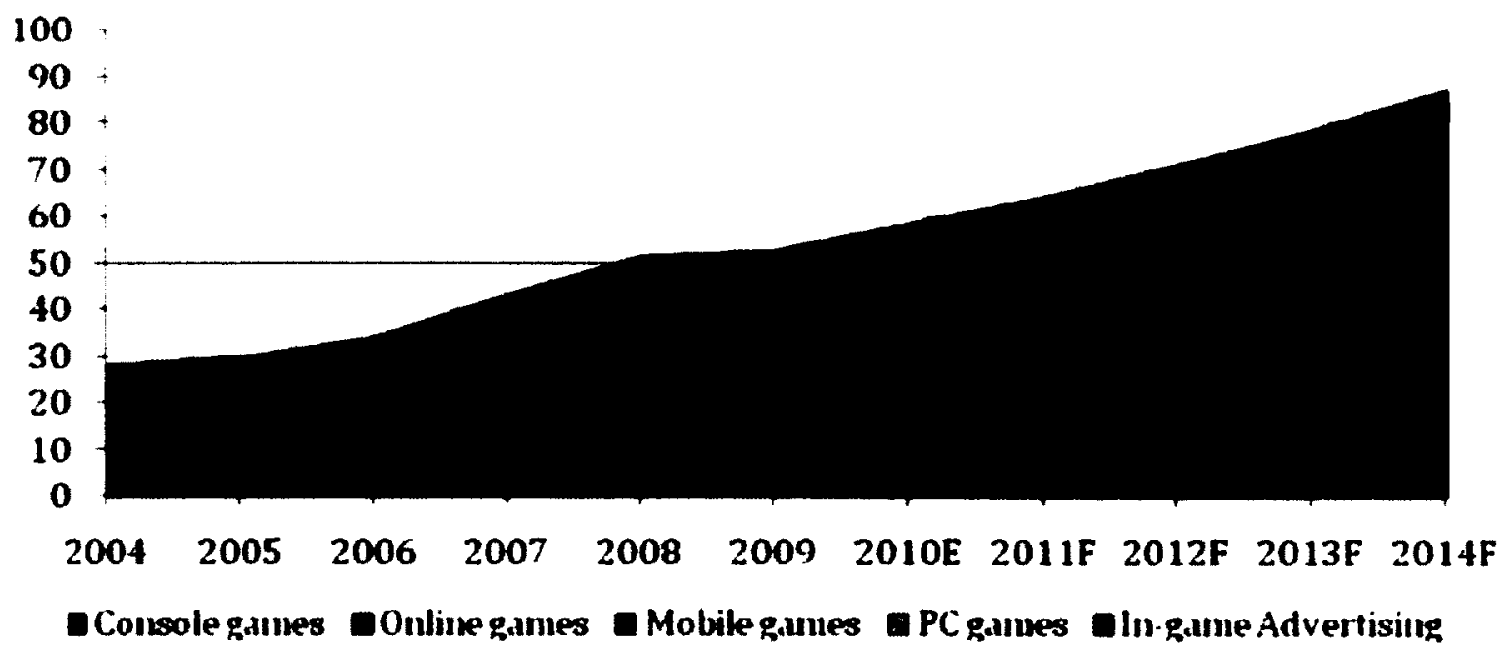

Source: (Merel 2011).

The games industry is experiencing a massive shift: moving from a predominately single-stream economic model dependent on retail PC and console games, to a more diverse economic landscape enabled by online connectivity and digital distribution. This industry-wide shift to digital distribution is not surprising. For example, Jennifer Johns predicted the impacts of growing digital distribution in 2006. She argued that digital distribution opposed the gatekeeping imposed by console hardware manufacturers, and would lead to a future "weightless"126 game economy in which consumers downloaded games directly from a variety of sources. Console manufacturers would lose the profits generated by their monopoly on physical game production, and therefore their control over game content and production would weaken dramatically (2006:176). The future predicted by Johns is now here, and the structure of production and access to finance and distribution are radically altered by online connectivity, and the promises of the New

126Johns argues that while digital distribution alludes to 'weightlessness', the realities of game development continue to be rooted in geographical and temporal space. 
Spirit of Capitalism.

\subsection{Detailing the 2011 Game Development Landscape}

In this section of the chapter, I introduce the Casual sectors, setting the stage for a more detailed discussion of casual, social and mobile games in the following chapter. I then describe how each of the Core sectors are adapting to the innovations popularized from the Casual sectors. I conclude the chapter with a discussion of how migrating to new sectors promises an escape from the constraints of traditional production methods, but introduces a whole new set of constraints.

\subsubsection{Casual}

Casual $^{127}$ games, unlike console development, are open to any developer, although certain casual platforms (mainly Apple's iOS) may require the game to first pass a certification process. In the following chapter, I more fully detail the economic models of casual games along with the related subsets of social and mobile games. But I will spend a few paragraphs here outlining key differences between the three sectors. Like casual games, social and mobile games are Casual in their play style. The difference between these three sectors is the distribution platform. The distribution streams for casual (little "c"), social games and mobile games differ slightly - casual games are generally distributed through online "portals". These portals, such as Big Fish Games and PopCap Games offer a range

127To cut-off the inevitable confusion: While casual games are a genre of games in themselves, the term also refers the the larger category comprised of casual, social, and mobile games. In industry discourses, "Casual" is most often used in the latter sense, especially in contrast to "Core", and thus encompassing casual, social and mobile games. The subset of casual games is increasingly ignored in industry discourses in favour of the newer categories of social and mobile games. 
of games that can be played in web browsers (most often these games are free, and are supported by advertising revenue), as well as games that can be downloaded (these games offer free trial periods, after which one must purchase the game to continue playing). Assuming traditional publisher functions such as advertising and distribution, portals act as a clearinghouse for casual games. Moreover, these portals also serve as a social networking site that allow players to share opinions and information about games and build communities (Consalvo 2009a).

Social games, in comparison, are distributed on social networking sites such as Facebook, GREE, Hi5, and MySpace. ${ }^{128}$ While casual game portals often take on marketing and advertising functions, social networking sites do not. Social games automatically advertise themselves using game play incentives that encourage players to disseminate the game to others. Accordingly, marketing budgets (normally the lion's share of development costs) are reduced. Social games are marketed virally via techniques that use a players' pre-existing social networks to expand awareness of the game. Simple 'word-of-mouth' can attract tens of thousands daily users just days after a game is launched, without spending any money on marketing (Sheffield 2010). With advertising budgets added to viral marketing, social games can attract millions of players a day. For example, a recent Zynga Game, Treasure Isle, went from 0 to 9 million users in six days (Sheffield 2010).

Mobile games are played on mobile phones and tablets and distributed through mobile platforms, such as the Apple App Store or Google's Play. The mobile market is

128As a side note, Facebook originally did not take any revenue share from developers, meaning that developers kept $100 \%$ of their profits. This directly accounts for Facebook's early success in attracting social game developers, and contributes to its current dominance in online social games. 
currently dominated by the Apple App Store. In contrast to iOS mobile games, the Android market is limited for a number of reasons. Unlike iTunes and Apple users, Android and Google users are not accustomed to paying for software. Android games are dispersed between a number of platforms, including Google Play, GetJar, and Amazon. This accordingly limits the discoverability of games, as - unlike Apple - all games are not available under one roof. Finally, Android purchasing functionalities lack the polish of iOS games, such as the inability to make one-click purchases within the game (a key design for increasing monetization and microtransaction revenue). ${ }^{129}$

While demanding less industry attention, casual gaming has, by far, the largest player population. For example, a survey of gamers in the US conducted by NewZoo reported that 113 million Americans played games on casual websites, in comparison to 87 million social gamers, and 76 million mobile gamers. Illustration 8.3 breaks these numbers down further, showing the overlap between the different sectors.

While many Casual gamers play games on casual websites, mobile devices and social networks, the data in Illustration 8.3, below, shows that casual games are, by far, the most popular sector, with an estimated 113 million players in the United States alone. Casual games can now be played on many different platforms, but the sector originated in games that came pre-installed on PCs, such as Minesweeper and Solitaire. They later moved onto the internet, are were accessible via casual game portals. Casual games generally refer to shorter, single-player games that prioritize matching sets, finding

129The Amazon store has clear advantages in terms of filtering games and recommending a game based on player's past purchases, drawing from its filtering system for book recommendations. However, Amazon has a poor reputation with developers. Amazon's contract terms have been flagged by IGDA, especially Amazon's ability to drop prices or give games away for free without developers' consent. 
hidden objects, and solving puzzles, for example Peggle, Plants vs Zombies, and Bejewelled.

Illustration 8.3: The reach of casual sectors in the U.S.

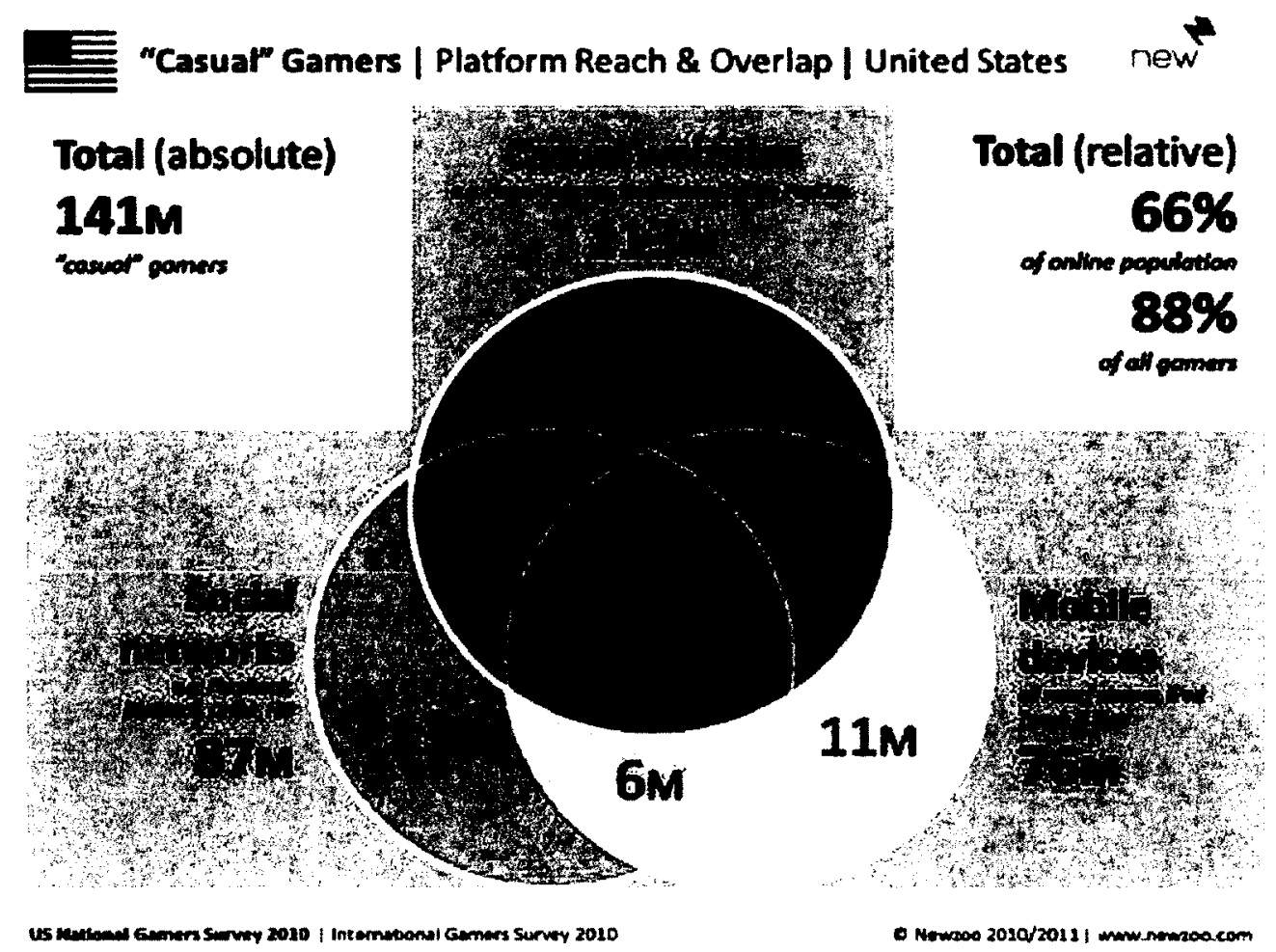

Source: (Caoili 2011)

In terms of style and design, casual games gave birth to social games. Like casual games, social games cater to larger player populations and encourage short play sessions that fit within the spare moments of a player's daily routine. The main innovation of social games is being embedded within social networking services (SNS) such as Facebook, allowing players to easily share the game and play with people that they know. Accordingly, social games add the social graph to casual games. Social games are 
updated frequently. ${ }^{130}$

Mobile games combine the accessibility of casual games and the social graph of social games with the convenience of always carrying the game in one's pocket. Mobile games are currently experiencing the greatest growth of any game sector. For example, the Entertainment Software Association states that $42 \%$ of heads of American households report they play games on wireless devices such as a cell phone or PDA, up from $20 \%$ in 2002 (Entertainment Software Association 2010).

While each of these sectors share similarities in terms of theme, accessibility, and short interruptible play sessions, differences between the distribution platforms leads to differences in how the games are created and played. Casual games are predominantly single player games. Each game takes minutes to a few hours to complete. In contrast, social games can be played solo, but are predominantly focused on encouraging players to invite their friends (already on the social network) to join the game. The novelty of interacting with friends, even asynchronously, leads to increased competition and player retention. Social games generally have no end point or failure conditions. Each player has their own small persistent game space that they add to, refine, and upgrade during weeks and months of play. Mobile games show more diversity in their design, especially since popular casual games (e.g. Plants vs Zombies) and popular social games (e.g. FarmVille) are ported to mobile versions. Some mobile games take advantage of the functionality of mobile hardware, utilizing the tilting motions enabled by gyroscopes, or the GPS built into smart phones Given the ubiquity of smart phones, the mobile sector is one currently targeted by developers.

130For example, Facebook games developed by Crowdstar are updated twice each week (Nutt 2010). 


\subsubsection{Core}

The widely broadcast success of games such as FarmVille and Angry Birds has garnered much attention from the game industry proper, with many publishers and developers from Core sectors, such as EA, now attempting to diversify and create Casual games for these new sectors. They have adapted their own practices in response, applying many new innovations from emerging sectors to their own. There is slippage between Core and Casual with innovations from each side influencing each other. This slippage increases as developers in the console and PC sectors attempt to woo the growing population of Casual gamers, and developers in the social, mobile, and casual sectors attempt to attract the fiercely loyal (and profitable) Core gamers. Publishers have slowly shifted their own practices to include these new sectors, adapting their production and revenue models, or buying out and amalgamating successful Casual game companies (Thomsen 2011). The following paragraphs detail how the Core sector is adapting and seeking to ameliorate the constraints of the production model described in Chapter Six.

\subsubsection{Console}

The console sector faces considerable challenges, including rising development costs and shrinking financial returns. Manufacturers and large publishers such as Activision and EA are slow to change in comparison with smaller, more agile development companies. However they have evolved since Kerr's analysis, spurred by the online connectivity offered by 7 th generation hardware.

Online connectivity has precipitated a shift in the business models of Core sectors, 
enabling a change in customer relationships for Core and PC games. In the traditional product-oriented model, games are sold on store shelves. They are a one-off product to be consumed, with a "ship it and forget it" mentality on the part of publishers and developers. The relationship between player and developer is indirect, only through the static game. This relationship is changing due to 'connectivity' and the use of metrics. Game companies are able to directly link to the customer in a more service-based orientation. For example, Jason Holtman, Valve's Director of Business Development, champions new business models for Core development premised on digital distribution that share many traits with Casual gaming models (Holtman 2009).

Online connectivity enables a service-based model that fosters long term relationships. Developers directly communicate with players through patches, content updates, and developer blogs. Players communicate with publishers and developers through fan sites, forum posts, and reviews. On an impersonal basis, developers and publishers watch what players do within the game via the collection of real-time metrics, a topic expanded upon in Chapter Ten. The ability to update, change, and add to games online extends their life cycle far beyond a few weeks on retail shelves. Online purchases for new downloadable content (DLC) extends this life cycle even further. Longer, more predictable profit periods decrease financial risk, a prospect that attracts industry investors (Holtman 2009).

This leads to a shifting economics of risk (Della Rocca 2009). In traditional game design, developers and publishers spend money until launch. Recouping these investments is restricted to the few short weeks the game is on store shelves, before it is 
heavily discounted. The influx of money comes only after the development cycle has ended. Ideally, the game sells enough copies to recover its expenditures, while the rare hit game may sell enough copies to fund future productions. Microtransactions have changed these economics. Developers are able to release a game in "beta" (a playable, but unfinished state). Microtransactions allow players to purchase additional items. If the game attracts enough paying players, the microtransaction profits can be rolled into further development, accordingly development of a larger, polished game is done in parallel with the growth of the playing population.

This model reduces economic risk, in that game concepts that initially fail to attract players are abandoned at relatively little cost (i.e. the losses would only include the time spent bringing the game to beta, rather than the larger costs of bringing the game to a final polished version). Instead of end-loading profits to a period of a few weeks, profits from microtransactions trickle in throughout development. This model, however, is closely tied with increasing precision and prediction of player behaviour. In order to reduce risk and determine how to improve the game, thus attracting more paying players, developers increasingly rely on surveillance in the form of metrics to guide their future steps, a topic that is explored further in Chapter Ten.

The shift to service is not the only adaption Core has made in response to online connectivity. Current industry profits are facilitated by the creation of smaller, downloadable console games and downloadable content (DLC). ${ }^{131}$ The difference

131To discuss the evolution of the downloadable and DLC sectors, I rely on Electronic Entertainment Design and Research (EEDAR). EEDAR is a market research organization that partners with companies such as Neilsen. It has amassed a proprietary game database with 20,000 games, broken down into more than 20,000,000 game features. This database is the foundation for all of EEDAR's services. EEDAR collected sales data on every downloadable game and DLC for all 7th generation consoles and handhelds and charted their growth over time. EEDAR extrapolates from this data, predicting that 
between downloadable games and DLC is that DLC is a supplemental add-on to a previously purchased full-size game. WiiWare, Xbox Live Arcade and PSN all offer high quality games that have passed console manufacturers' internal screening and quality assurance processes. Generally, these downloadable titles are shorter in length (e.g. 4 hours of game play versus 40 ) and are less expensive to purchase (e.g. $\$ 5-\$ 15$ rather than the $\$ 50-\$ 70$ for a retail game), echoing many of the successful traits of Casual games. There are numerous benefits of downloadable games, including the ease of distribution. Players can download the game on demand without having to trek to their nearest retail location.

On the developer side, digital distribution means there is limited overhead, cutting out the costs associated with the physical manufacture and distribution of the game. Ultimately, this means that both publishers and developers reap a larger share of the profits. For example, for a typical $\$ 60$ physical game, approximately $\$ 24$ goes to the publisher and developer. The remaining $\$ 36$ is divided between the manufacturer, distributor and retailer. ${ }^{132}$ In contrast, the average cost of a downloadable game in 2010 was $\$ 10, \$ 3$ of this went to the console manufacturer to cover "networking fees" while the remaining $\$ 7$ went directly to the publisher/developer.

downloadable games and DLC's revenue will grow over 35\% in 2011, and by 2014 will comprise 25\% of console software sales (Electronic Entertainment Design and Research 2011).

132Input data was amalgamated from both Kerr (2006:66) and Johns (2006:165), and aligns with more recent numbers from Electronic Entertainment Design and Research (EEDAR) (Electronic Entertainment Design and Research 2011). 


\section{Illustration 8.4: Profit distribution for retail vs downloadable games}

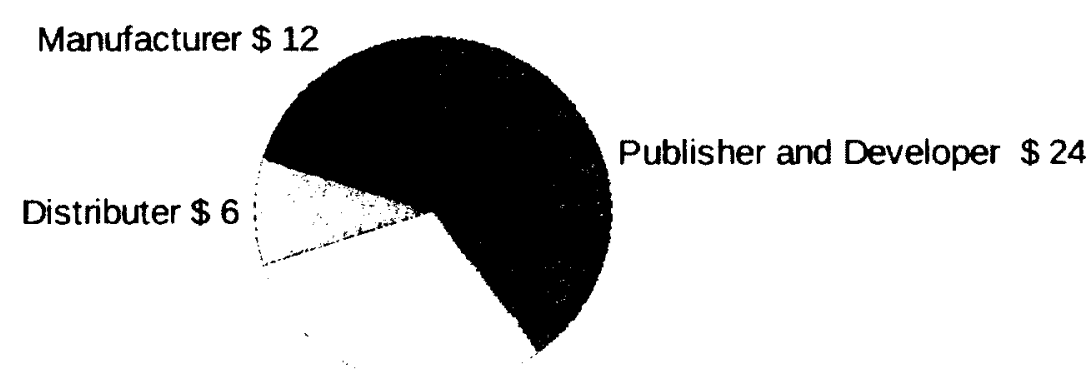

Retailer $\$ 18$

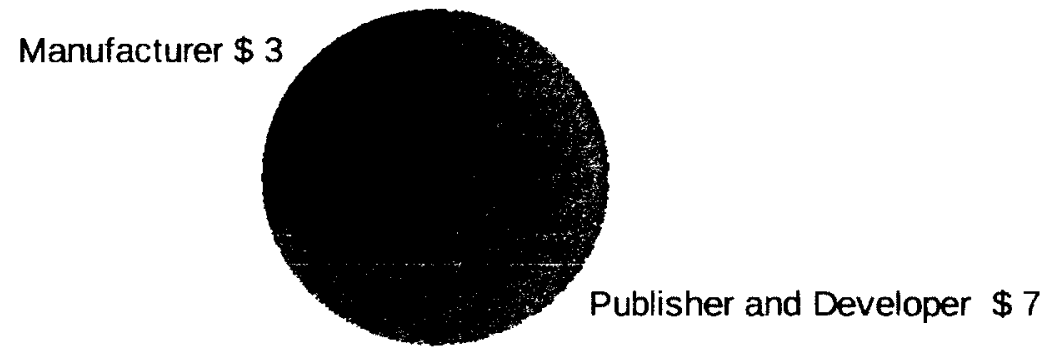

Digital distribution fundamentally changes the profit ratio that has, up until now, characterized console development. With the shift to downloadable console games, $70 \%$ of the revenue goes to the publisher and developer, instead of the traditional share of $40 \%$. Although downloadable games are generally priced lower than retail games, they are also created more quickly and with smaller budgets. In addition, their average price is steadily increasing (Handrahan 2011b). Downloadable games also offer other advantages. Because they are linked to the player's purchasing account on the console, this means they cannot be resold. This linkage also hinders game piracy. ${ }^{133}$

133Full-sized console games are increasingly downloadable. But larger games take much longer to download and consume more of the player's limited internet bandwidth. This increases the risk of players having to pay ISP fees for going over their allotted download quota. These factors are compounded by the fact that full downloadable games are generally not any cheaper than the same physical game offered in stores, and are generally more expensive than used copies. Players thus weigh the convenience of downloading full games against the ability to recoup the cost of the physical game 
In addition to downloadable games, DLC increases revenue flow, inspiring fans of the game to pay for individual packs of content, including new maps, additional equipment and weapons, and playable characters, many of which are especially attractive for multiplayer games as they may give paying players more variety and competitive advantages over players who do not purchase DLC. DLC also includes new levels, musical tracks and story lines.

There are additional reasons fuelling the growth of DLC. As enumerated by Daniel Nieborg (2011), DLC:

1. Extends the shelf life of the stand alone product;

2. Decreases trade-ins, and increases re-playability of the original game;

3. Allows the publishers to maintain the original game's pricing longer;

4. Eliminates sales return risks, as players cannot return DLC;

5. Combats piracy, as downloading DLC necessitates internet connections to the publisher, allowing them to simultaneously install piracy counter-measures; and

6. Gives publishers a better picture of who their customers are, what kinds of DLC they buy, why they buy it, and what they have already installed.

As a side note, used game sales are particularly detrimental to the game development industry, as profits from the sale of trade-in games go directly to the re-seller and are not shared with the publisher or developer. Offering DLC is one method to prevent the resale of games, as players delay selling their game if they know that future DLC will be released. Additionally, DLC can be used extract profit from used games sales as players purchase DLC for their second-hand games.

The console downloadable market offers solutions to the constant ramping up and ramping down that epitomizes the console cycle. Traditional development cycles create resource challenges for game development studios. The conceptualization and pre-

by re-selling it when they are done with it. 
production phases are carried out with a small core team who plans the general shape of the game. In the production phase, this team acquires a large number of developers, rapidly "ramping up" (hiring) to peak production size. When the game is released at the end of the production stage the team is "ramped down" (firing). While studios would prefer to continuously employ developers instead of always hiring and firing, maintaining surplus labour costs studio resources and is not economically viable. Some development studios shift these now superfluous employees to other internal projects but this is not always the case. This contributes to a lateral movement between studios as employees who have been "ramped down" look for employment in other studios that are "ramping up". Accordingly, traditional console development work epitomizes precarious labour. Downloadable games and content alter this cycle. Instead of firing employees after a game is released and retaining only a core team to plan the next game, studios increasingly see downloadable console games as a stopgap between the peaks of development, allowing them to retain more skilled employees during the down periods between AAA games, as depicted in Illustration 8.5, below, which was adapted from (Gouglas et al. 2010:10). 
Illustration 8.5: Integrated console and downloadable game development cycle

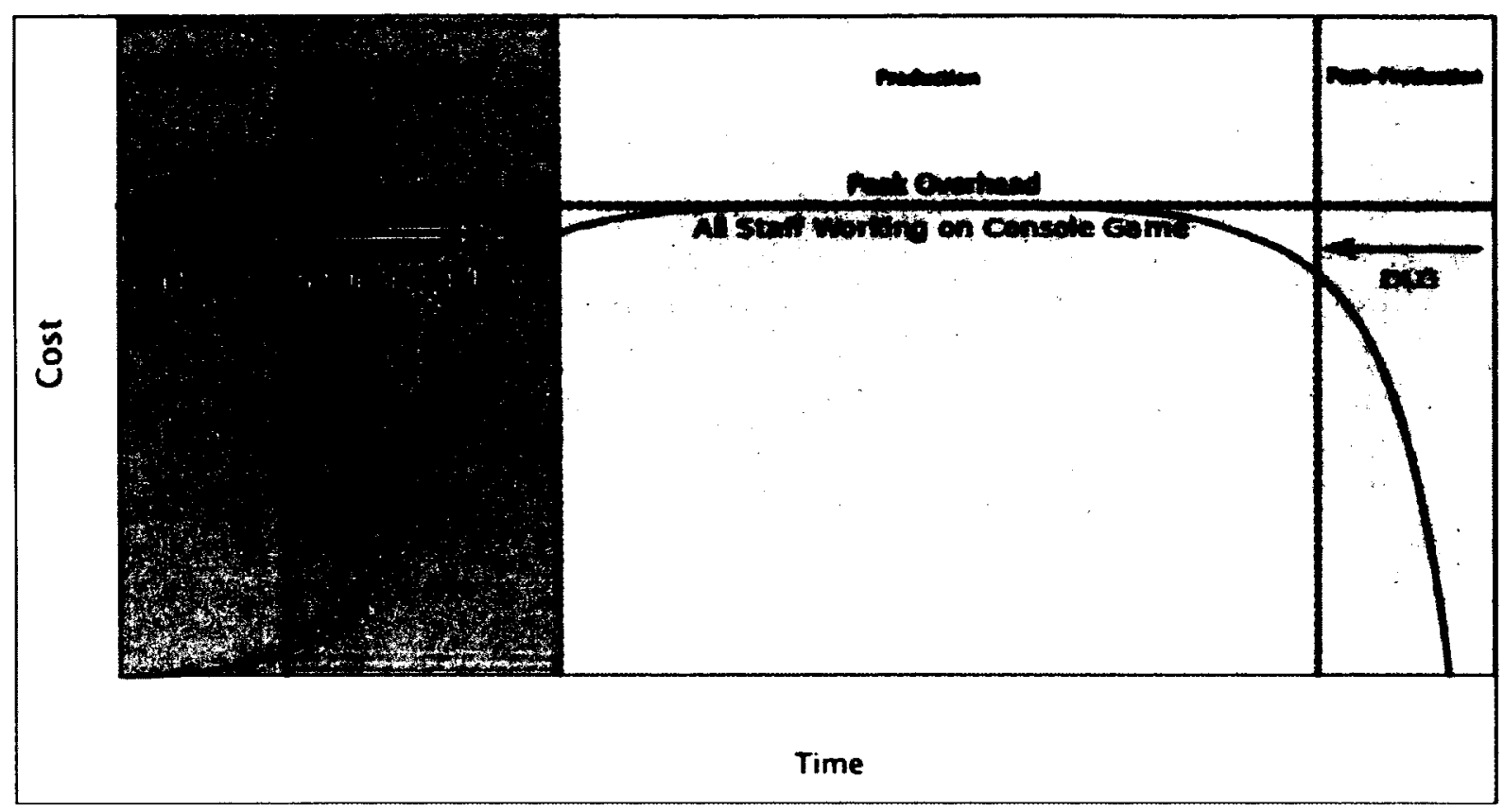

Source: revised from (Gouglas et al. 2010:10).

Considerable support for downloadable development is attributable to the success of studios such as Double Fine (Nutt 2011d; Parkin 2010b). During a lull in production of Brutal Legend, the studio experimented with the creation of smaller game concepts. After Brutal Legend shipped, in the downtime between projects the studio turned the experiments into fully-polished games. With smaller budgets of $\$ 1-2$ million rather than \$25 million, Double Fine was able to take more creative risks. According to Tim Schafer," You're either making really big expensive games that take no risks or make less expensive games that are creative and original" (Nutt 2011d). Smaller games also meant that the studio had a greater chance of retaining its profitable IP rights. In two months, its four downloadable projects were signed with publishers (the final of the four, Once Upon 
a Monster was released fall 2011). The projects were so successful the studio does not plan to return to AAA development, and is currently experimenting with the alternative crowd-sourced funding method, Kickstarter. ${ }^{134}$

Ultimately, an increasing percentage of the console market is digital, and more and more players are purchasing DLC and downloadable games. While these services offer an initial entry point for independent developers looking to break into console development (e.g. World of Goo, Braid, etc.), the downloadable market has become much more competitive as established console developers colonize the space (Vanden Bossche 2011; Martin 2011b; Elliott 2011c) and production costs increase.

\subsubsection{Handhelds}

The handheld sector faces many of the same challenges of the console sector. While development is shorter and less expensive, games are still relatively expensive to purchase at $\$ 20$ - $\$ 45$ per game. The sector has been decimated by the rising popularity of mobile games and the increasing technological sophistication of smart phones. Since iPhones were introduced in 2008, Nintendo's portable gaming revenue share fell from $75 \%$ to $57 \%$ (Valadares 2011). A direct competitor to handhelds, mobile phones offer a wide range of games that are easy to download and are much cheaper than handheld cartridges, costing a few dollars or less. While the development budgets for the newest generation of handhelds, the 3DS and PS Vita, have doubled or tripled, popular mobile games such as Angry Birds only cost $\$ 140,000$ to develop (Crossley 2011d; Graft 2011b).

134This experimentation was wildly successful. In the spring of 2012, Double Fine asked fans for contributions for their next game. They raised over $\$ 3.3$ million USD from over 87,000 contributors, well over the $\$ 400,000$ they originally asked for. 
The two main handheld manufacturers are innovating in different technical directions: Nintendo released the 3DS in March 2011, showcasing glasses-less 3D gaming. Sony released the PS Vita the following winter, aiming to mirror the high quality of Core gaming on a mobile platform. However, the disappointing reception of both handhelds does not bode well for the future of the handheld. ${ }^{135}$

Developers for handheld face numerous challenges: developing for unproven next generation platforms, which are already faced with a cool reception and poor software sales, rising development costs, and competition with similar games offered much more cheaply on smart phones. Instead of spending hundreds of dollars on a handheld with a limited number of games to choose from, consumers use their existing smartphones for gaming, especially when app stores already offer a vast selection of games numbering in the hundreds of thousands.

\subsubsection{PC - Full Size}

Historically, the only alternative to console development was developing games for the

PC market. At first glace, the PC market tended to avoid the marginalization of

developers given that it is free from the proprietary restrictions, licensing and

manufacturing fees, and the expensive development kits that characterize console

135Nintendo announced a significant price drop a few months into production ( $\$ 250$ to $\$ 170$ ), hoping to spur lacklustre sales (Plunkett 2011). Sony has attempted to combat the mobile market by offering a phone for gamers (Xperia Play), as well as integrating functionalities commonly found in mobile games; including location-based services that use GPS to promote online mulitplayer gameplay and sharing (e.g. players can find other Vita users nearby using the system's GPS functions), and a number of social networking applications (e.g. players are able to drop and share information, challenges, and digital gifts of varying rarity with other users). As of yet, however, the handheld sector has not embraced new freemium and F2P models. For example, Nintendo president Satoru Iwata has vociferously opposed free-to-play titles and games published on mobile and social platforms (M. Rose 2011a). 
development. PC development costs were lower than console development, meaning that PC games had a much lower break-even point. For example, in the early 2000's high-end PC games needed to make 90,000 sales in order to break even compared to 500,000 for licenced Nintendo games (D. Williams 2002:46). PC software also has a different profit distribution: retailers claim $30 \%$ of the retail value, distributors $10 \%$, and developers and publishers combined take the remaining $60 \%$, as no royalties are due to PC manufacturers.

However, the low barriers to entry mean that the PC market is much more crowded than console development and many more titles are released that vie for consumer's attention. As a result, the price point of PC games is typically much lower than that of console games. As put by Williams, while "consoles represent the mainstream of gaming, the smaller PC market represents the vanguard of imaginative programming, risk taking and fringe products" (2002:45). PC product diversity is driven not only by the low cost of production but also by demand of consumers. While console owners are generally younger on average, male, and have a preference for competitive action and shooting games, PC gamers are much more demographically diverse.

Williams (2002) notes that, despite the ease of entry and room for innovation and experimentation, many developers prefer the stability of console development with its well-defined parameters. Partly due to the fact that the hardware and software configurations of players' computers vary widely, PC games are also more likely to ship with software bugs and glitches. In comparison to the marketing efforts underwritten by console manufacturers, PC games are not as heavily marketed or funded. Publishers 
prefer console games as the target market is homogenous (e.g. younger males) and thus consumer purchasing is easier to predict (Johns 2006:165). Veteran RPG game designer Warren Spector discusses the changing market for Core PC games:

There has definitely been a change even over the last three to five years, and I think it is a substantive change, not just a rhetorical one...it is harder and harder to find people willing to fund games that only go after that narrow hardcore audience. It is a real change, no question about it. It costs the same amount of money to reach 250,000 hardcore real-time strategy gamers as it does to reach potentially five million console gamers. (Juul 2010:204)

Because of these reasons, the demise of the Core PC market has been predicted numerous times (Radd 2011b). Console games garnered larger development budgets and marketing funding, thus limiting the reach of PC games. Not surprisingly, most PC games are ported to console ${ }^{136}$ (e.g. Call of Duty and Crysis franchises) and many are now remade for social networks, including well-known franchises such as The Sims and Sid Meier's Civilization. The most popular PC-only games fall into the MMO category (e.g. World of Warcraft and Rift), with only a few AAA PC-only games (e.g. Starcraft In) remaining that aren't MMOs.

Despite the bleak outlook for retail PC games, the downloadable landscape is flourishing, partly due to to the existence of digital distribution platforms such as Steam, Kongregate, and Impulse, which act as clearinghouses for PC games of all sizes. The example of Steam highlights the utility of these platforms. While commonly associated with Valve's own slate of core games (e.g. Team Fortress 2, Half Life 2, and Portal 2),

136While PC and console both cater to Core gamers, these communities are often opposed to each other. The cutting edge technology in PC gaming rigs can easily cost thousands of dollars, but most studios that make PC games also aim for console ports and the increased access to funding they attract. PC gamers thus argue that the quality of PC games is pulled down by the need to design them to be playable with less sophisticated console controllers and hardware. 
Steam hosts indie, casual, and console ports alike. Steam controls an estimated $70 \%$ of the $\$ 4$ billion market in downloadable PC games (Chiang 2011). In exchange for $30 \%$ of the sales revenue, the platform offers multiple services to developers. ${ }^{137}$ Other digital distribution platforms offer a similar, although less extensive suite of services.

Without the infrastructure and services provided by these platforms, many PC developers would not be able to find an audience for their games (M. Rose 2011c; Meer 2011d, 2011b, 2011e). Accordingly, developers are willing to give distributors a larger percentage of their game revenues. However, like console manufacturers, Steam is a gatekeeper, limiting the number of games on its platform. While players are reassured that any game on Steam meets a certain level of quality, many developers find their games rejected.

\subsubsection{MMOs}

Massively Multiplayer Online Games (MMOs) originated from Korea where console gaming was banned until 2001. The sale of consoles is still banned in China (ironically, since most console hardware is manufactured and assembled there). The lack of competition from the console sector allowed PC gaming to develop and grow within a protected market. At the time of Kerr's analysis, MMOs were subscription-based. Players

137These services include: access to large pre-existing communities of gamers; embedded social networking services that enable user profiles, instant messaging, and friends lists, thus facilitating social play; multiplayer lobbies and matchmaking that allow players to organize and agree on game settings before joining, and can automatically group players together based on certain criteria such as skill level; automatic distribution and downloading of game patches and updates; payment frameworks, including multiple payment options, and the ability to integrate microtransactions into games; anti-cheat systems that identify and respond to cheaters and pirates; data analytics services, including the collection of metrics on the usage and reach of game, stability and performance indicators, and sales data; and, perhaps most importantly, advertising and game promotion, including hosting frequent sales. 
purchased the physical game at shops and paid a monthly subscription to access to the online game world. Boxed expansion packs such as those for WoW, and DLC have added additional revenue streams.

The MMO market has been dominated by a relatively small set of games, World of Warcraft being the most well known, while Ultima Online and EverQuest, released in 1997 and 1999, respectively, are two of the longest running MMOs. The massive success of WoW attracted venture capitalists to the development of MMOs. Because MMOs boast a stable user base and long-term revenues that do not greatly fluctuate from month to month, MMOs are an attractive financial opportunity compared to console games, whose success is difficult to predict and whose revenues are concentrated to a few short weeks of retail sales.

Despite the initial investor influx, and a number of well-funded MMO development projects, a 'WoW- killer' has yet to be found. A reason for this lies in the temporal demands of playing MMOs, which generally preclude playing more than one MMO at a time. In 2006, researchers estimated that reaching the highest level of WoW took, on average, two full months of work days (Ducheneaut et al. 2006). Migrating to a new, untested MMO potentially means leaving friends behind, as well as the social capital, virtual goods, reputation, and emotional investment developed over hundreds of hours of playing (Humphreys 2007). In addition, newly released MMOs simply cannot match the polish and the sheer amount of content that has been amassed over years of adding to and refining WoW and other long-running MMOs. In additional to massive development expenses, MMOs incur substantial maintenance costs including those 
related to server space, the continual release of new content, community management, and balancing the game to ensure that different player classes and weapons are evenly matched. For example, Kerr reported that support staff costs for Ultima Online cost 1 million Euros annually, on top of the initial \$15 million Euro development cost (Kerr 2006:59).

In response to the pressure of competing with WoW, and following the economic success of Casual F2P games, an increasing number of Core games have successfully shifted from their traditional retail and subscription models to F2P, relying on microtransactions and the sale of virtual goods to generate profits. ${ }^{138}$ This shift to F2P is a successful one for many MMOs. For example, the MMO The Lord of the Rings Online shifted from a subscription model to F2P in October 2010 and tripled their revenues by January the following year (Meer 2011c). Other MMOs to make the shift include Dungeons and Dragons Online, and Everquest II. How this shift occurs differs according to the MMO. ${ }^{139}$

\subsubsection{Indie}

The growth and evolution of the indie sector is the most difficult to define because the

138The decision of what to sell greatly effects the game's balance. For example, some games follow the freemium model and sell items that give paying players a distinct advantage over non-paying players (e.g. more powerful weapons). However, many players see this as an unfair practice that prioritizes purchasing power over actual skill. Other games only sell goods that do not affect the balance of the game, for example aesthetic items that do not impact game play (e.g. monocles and clothing for avatars in MMOs like Eve Online), or items that save time, allowing players to move through the levels faster.

139Some MMOs eliminate subscriptions entirely (e.g. Global Agenda), relying solely on microtransactions and the sale of expansion packs for continued revenue. However, the most common shift is a move to allow free access to basic content, but reserving premium content for subscribers (e.g. EverQuest II, Dungeons and Dragons Online, and Lord of the Rings Online). If a player wants to unlock new content or areas (e.g. end-game raiding or more powerful weapons) they can pay a la carte, but the most exclusive content is reserved. Even WoW has given a nod to F2P, granting players free access to the game until they reach level 20 . 
term "indie" itself is highly contested (see Siegel 2010). Independent studios are those not owned by a publisher and hence able to choose which games they develop. The term "independent" includes large studios that create AAA console titles for hire. In contrast, "indie" can either mean a method characterized by small teams and limited budgets, or a style that is rough and artistically - rather than economically - driven, both of which are linked to full creative autonomy (Caoili 2010a).

Most indies speak the language of Core, sharing a love of games and many of the same values of console and PC developers. They just may not share the vision of what creates a successful game (e.g. disdaining 'selling out', or working as a cog in a massive team). This stands in contrast to developers from emerging sectors such as social games who have come from different backgrounds and cultural milieus (e.g. web design, marketing, etc.) and thus may not be conversant in this language. The ties between the PC and console sectors and these sectors (e.g. social, mobile, and gamification) are accordingly much weaker, although they are strengthened as veteran developers are recruited into these lucrative sectors. If we see the selection of what postmortems get published in Game Developer magazine as an indicator of what developers and games belong within the game industry proper, indie games commonly appear in postmortems, while mobile, gamification and social games are markedly absent.

While over half of indie developers have never worked in mainstream development (Carmel 2010), there is a tight relationship between the indie and the Core console and PC sectors. Some indie developers aspire to work in traditional PC and console sectors, while others turn indie specifically in response to the frustration of 
working within traditional sectors. The lifespan of an indie developer's career is short. Developers that release successful games (i.e. popular, financially lucrative, or critically well-received) are targeted for acquisition by large publishers, while less successful developers go bankrupt and thus seek more stable employment in larger companies or outside of the industry altogether. In the opposite direction, many employees leave mainstream development looking to bootstrap their own indie studios with the skills and knowledge gained from working within the mainstream industry. Accordingly, the churn of indie developers fuels the creativity of larger mainstream studios as indie personnel come and go.

Self-identified indie developers create games for every sector except large MMOs and console games (the costs of development being prohibitive). Console manufacturers have attempted to leverage the creativity of indie developers by offering channels such as PSN, WiiWare, XBLA to release downloadable indie games alongside the games of larger publishers, after subjecting them to a rigorous certification process. Microsoft even offers a channel for indie-only games, Xbox Live Indie Games (XBLIG), while PC indie games are often released through distributors such as Steam and Kongregate, or on developers' personal websites.

However, due to intense competition it is increasingly difficult for indie developers to get their game on elite platforms such as Steam, PSN or XBLA (Deejay 2011). As stated by indie developer Sean Murray:

I think without the support of at least one of those outlets, the chances of success are reduced from tiny to almost non-existent. I think that if you talked to other indie developers, they might say, we've been turned down by Steam, or XBLA or PSN. It's a really common thing to hear, but it's also often a real 
finishing blow for any studio. (Pearson 2011)

XBLIG, while developed expressly to showcase indie games, has received considerable recent criticism. While XBLIG makes it relatively easy for indies to publish a low-cost console title, XBLIG games have been hidden away on the console interface. The relative lack of gatekeeping and promotion means that the same games released on platforms such as Steam are much more profitable. For example, the average game on XBLIG makes only $\$ 3800$ (Webster 2011c), and sales from less then a week on Steam can match the sales of an entire year on XBLIG (M. Rose 2011c).

Mobile distribution is characterized by far fewer gatekeepers. However, indie success is predicated upon attaining visibility in an extremely crowded marketplace. For example, without large marketing budgets and the support of Apple (i.e. being selected as a featured game), most new iOS games are soon lost amongst the millions of other available apps. Despite the drawbacks of distribution platforms for PCs, console downloadables and mobile games, they are still better than the traditional console oligopoly. As stated by Sean Murray:

I think they [online distribution platforms such as Steam, Apple, or XBLA] are also in complete control of that scene, in complete control of the output of indie developers. It's kind of a scary place, but I think we're certainly lucky to have them. If you look at the last generation, you couldn't make a PS2 game unless you were with a publisher and had a pretty sizable team. There were no other options. (Pearson 2011)

A number of alternative funding sources have been established for indie developers (M.

Rose 2011b; Carmel 2010), including crowd-sourcing production costs through donations via sites such as KickStarter (Webster 2011b; Gasse 2011; Crossley 2011a). The numerous publishing options for indies fuel claims about the expansion of indie games, 
especially with the well-publicized successes of games such as Minecraft. ${ }^{140}$ Despite the widely-publicized incomes of indie developers such as Notch, roughly $55 \%$ of independent game respondents working on a team or on their own reported making less than $\$ 500$ from game sales in 2010 (Newman and Sheffield 2011). While there may be a indie resurgence driven by digital distribution, this resurgence does not automatically equate to economic stability for indie developers.

\subsection{Situating Industry Shifts as Displacements in the Spirit of Capitalism}

Instead of attempting to enter the console wars and compete with industry giants such as Microsoft and Nintendo, new emerging sectors (casual, social and mobile developers) leverage online connectivity, aiming for a different market altogether and creating a very different product (thus the commonly heard refrain from Core developers that these aren't 'real' games). Accordingly, these new sectors effectively snuck into game development under the radar, away from the competitive marketplace dominated by console manufacturers and established game publishers. In this sense, emerging sectors and adapting sectors demonstrate the displacements and tests as one Spirit changes into another.

Echoing Boltanski and Chiapello's (2007) findings, industrial logic in game industry discourses still predominates. But networked logic is becoming more and more common, exhibiting changes in how the industry is being thought of and talked about, as well as concrete changes to what it looks like at ground level. We can situate these

140Developed by Markus "Notch" Persson, the sandbox game Minecraft amassed $\$ 33$ million US in sales while still in the unfinished beta version (Orland 2011b). 
changes on an individual level (i.e. not just macro-economic or technological) by explaining how people commit themselves to action, their motivations, and the meaning they give to their acts. Boltanski and Chiapello argue that the justificatory regimes of Cités provide the foundation for these explanations, thus tying together the individual agency of game developers to the massive structural changes the game industry is undergoing.

Cités are temporally and spatially rooted in their historical contexts, identifying and generalizing a form of existence that supports "a definition of the common good and a criterion for judgements about the value of beings, according to the contribution they make to the good of all thus conceived" (Boltanski and Chiapello 2007:520). In other words, each Cité presents a model of how the world works, what is the 'good life', and how to achieve it. New Cités are established in times of significant social change, legitimating new 'worlds' as well as the new forms of inequality and exploitation that go along with them. Cités aim to make these new worlds more just, by subjecting actions to tests that limit exploitation and restrain the actions of the powerful. Once Cités and the tests that go along with them are established, a more ordered (i.e. fair) world displaces a chaotic universe wherein the strong exploit the weak. Cités undergird both justice and critique. Each Cité can be used to criticize the tests organized in accordance with the logic of other Cités, as well as criticizing the negative practices that are pertinent from the standpoint of the Cité itself.

Cités change over time though a series of tests and displacements. Tests occur when Cités come into contact with the real world, applying the theoretical construct of 
justice of each Cités to material examples. Simply put, Cités promise individuals: "If you do X, you will succeed. Your actions will be fair, and you will have contributed to the common good, as defined by this Cité's description of the world, how it works, and the values that underlie it." As we saw in Chapter Six, the promises of the Industrial Cité are harder to keep in light of steadily decreasing profit margins, studio closures, and massive firms that are too hierarchical to adapt to and take advantage of technical innovations (i.e. smart phones, social networking sites, etc.). Accordingly, the industry is ripe for change.

Tests are ways to prove and uphold the promises of each Cité. Boltanski and Chiapello outline two forms of tests. "Legitimate tests" are measured according to the tenets of the particular Cité model within which it falls. They are qualified, categorized and formally regulated to make the world 'fair', to constrain the progress of capitalism, and to ensure that stronger members cannot exploit weaker members. Legitimate tests are judged by other people, based on explicit and acknowledged principles of equivalency, whereas tests of strength ignore these rules and regulations. In tests of strength, the ends justify the means. Anything goes as long as it is met with success.

Displacements are a response to these tests of strength. These displacements involve:

[G]roups of actors trying to free themselves from that which keeps them from enjoying and from increasing their privileges. They do this by looking for new ways to succeed and to gain recognition without having to take currently legitimate tests. Such actors tend to avoid established tests by experimenting with hazardous, local, and often low-key displacements. (Boltanski and Chiapello 2005:172).

All of a sudden, people succeed in a different fashion. Each successful displacement gradually modifies the test system, substituting new, less formalized tests for the 
previously established ones (ones that are often constrained by legal regulations). For example, making a profit by trying something novel passes a test of strength. The business models described in this chapter are all forms of displacement. Instead of creating a physical game to be sold though a retail store, online connectivity and digital distribution have allowed developers new (successful) ways to generate revenue, from microtransactions, to extra downloadable content, to providing a service rather than a product.

Displacements dispense with conventions, with established ways of doing things. Displacements become the locus of new forms of injustice and exploitation, "facilitated precisely by the fact that they were based on tests that had not been identified and categorized" (Boltanski and Chiapello 2007:345). For example, the predominance of outsourcing and the delocalization of studios to regions with cheaper labour or extensive tax incentives are two earlier displacements in the game industry. This chapter has detailed a number of displacements, such as moves to eliminate developer's reliance on publishers and manufacturers and new distribution methods that eliminate retail providers. These displacements are tests of strength, attempting to circumvent traditional console models and finding new routes to success (measured by increasing player numbers as well as developers' profit share). As we will see in the following chapter, displacements in social games such as a/b testing and viral marketing to engage players, can be seen as new forms of injustice and exploitation (at least according to the game developers who are criticizing social game companies). However, displacements such as these "disarm criticisms by presenting critics with a world that they no longer know how 
to interpret" (Boltanski and Chiapello 2005:175).

This cycle of tests, displacements, and the adoption of new Cités in response to overwhelming displacements explains how capital absorbs and responds to criticism. It thus situates the traditional console model of the industry, as rooted in the Industrial Cité, but facing numerous small displacements that attempt to make the lumbering industry more agile, more innovative, and capture some of the successes of the Casual sectors. However, as we will see in the next chapter, the Spirit of Capitalism rests on cycles of recuperation. As put by Boltanski and Chiapello:

Capitalism attracts actors, who realized that they have hitherto been oppressed, by offering them a certain form of liberation that masks new types of oppression. It may then be said that capitalism 'recuperates' the autonomy it extends, by implementing new modes of control. (2007:425)

With each displacement from Core towards Casual modes of production, game developers are finding new freedoms, but also new masters and new forms of oppression.

\subsection{Conclusion}

This chapter mapped out the game industry landscape, examining a range of game development sectors and focusing, in particular, on how Core sectors are adapting in response to emerging Casual sectors, online connectivity, and digital distribution. It situated these adaptations as displacements within the largely industrial modes of production that characterize the traditional console development process, as Core developers look to emulate the success of the Casual Revolution.

As we will see in the next chapter, these emerging sectors are even more tightly rationalized and instrumentalized than traditional development, emphasizing precision, 
rules, and efficiency. Games from these emerging sectors, while promising developers more freedom, are closely tied with the rationalization of play. Play and creativity are increasingly quantified and put to instrumental purposes that are predicated upon feedback, and closely monitoring and channelling players' actions in order to reduce the financial risk inherent in game development and to increase the amount of money players are willing to pay. They become surveillance games: by knowing what players want or what they spend time or money on, developers can hone their focus and avoid wasting resources on other assets or mechanics. Ideally, this would reduce financial risk and allow developers to experiment with new and innovative game ideas. However, in practice, game play in emerging sectors is highly scripted and constrained, each level, quest, and mission planned in advance by developers. On a theoretical level, the examination of emergent trends in the production, development and distribution of games highlights how both games and play are continually morphing in response to economic constraints and technological innovation. 
Part III.

Welcome to the Projective Cité 


\section{Chapter 9. New Economies of Play: Casual, social and mobile games}

This thesis uses the New Spirit of Capitalism as a framework to more broadly situate and explain the current shifts in the game industry. In particular, the struggles of the console industry and the rapid emergence of social and mobile game sectors can be located in terms of Core sectors - premised on the industrial models developed during the Second Spirit of Capitalism - attempting to compete with Casual sectors, whose structure and operation embodies the New Spirit of Capitalism. This chapter asks: If traditional console is equated with the Second Spirit of Capitalism, what does the New Spirit of Capitalism look like in the game industry? It answers this question with a case study of social game development, comparing it to the console development detailed in Chapter Six. It explains how these agile models of production ameliorate the concerns of Industrial Cité, but ignite new concerns relevant to the Projective Cité. It also describes some of the the innovations to the New Spirit of Capitalism that were not predicted by Boltanski and Chiapello, mainly the reliance on metrics and predictive modes of governance.

While the Projective Cité operates on connectionist logic, emphasizing autonomous individuals who find success through the fruits of their creative labour, a deeper look at social games tests these promises. Developers, as we will see, are promised a creative workplace free of the constraints that characterize console studios, but this promise fails to materialize due to other displacements. Instead of being guided by the visionaries and "great" leaders of the Projective Cité, social game development manages the uncertainties and risks of the industry by capitalizing on newer trends in 
networked-based thinking, such as analytics and crowd-sourcing In doing so, social game development re-establishes the rationalization, instrumentalization, and calculations of efficiency of the Second Spirit, but in a new, objective, quantitative way that relies on customer data rather than bureaucrats (i.e. console manufacturers and publishers) to drive decision-making. Thus, this displacement is much harder to criticize because it comes bottom-up from countless consumers, rather than being imposed top-down by management hierarchies.

Much of the primary data for this chapter came from interviewing developers in Casual game development and from viewing game developer conference sessions - both in person, and via the GDC Vault (www.gdcvault.com), which records and archives all sessions for later viewing. ${ }^{141}$ These sessions were particularly illuminating as - more often than not - they evidenced Casual developers' attempts to join the game industry proper. These developers fervently espoused the benefits of Casual economic and production models, as well as their commitment to making 'good' games; however they were often met with rejection. Only as more and more developers shifted from Core to Casual did this rejection lessen (see Brathwaite 2011b). Other primary sources included developer blogs and other online sources (see Appendix C) that illustrated the resistance to Casual.

\subsection{Defining Social Games and their relationship with Casual and Mobile}

Virtually unheard of a few short years ago, social games - a subset of the larger category of Casual games - are now seriously altering the composition of the games industry.

141Passes for GDC conferences easily exceed $\$ 1000$, far outside of the budget of most academic researchers. 
Resources and personnel are moving from mainstream development to Casual, creating massive shifts in the industry. As we will see in this chapter, developers of Casual games may face constraints in the forms and genres of games they make, but they lack many of the overwhelming constraints (financial, scheduling, hierarchical oversight) faced by Core developers. Importantly, Casual games are drawing resources from outside of the games industry: attracting players who do not consider themselves gamers, drawing funding from investors who would never invest in traditional development, and attracting developers who have roots outside of games.

The rise of Casual games ${ }^{142}$ in general, and social games in particular, was unanticipated by most video game industry insiders (Schell 2010b). However, in hindsight, the rapid emergence of mobile, social and casual games since 2006 is not surprising given the numerous constraints on development listed in Chapter Six. Games from the Casual sectors are the epitome of accessible. They are offered free or at negligible cost and play on the cell phones and computers that users already own, therefore diminishing any technological or financial barriers to entry. Development barriers are also low (made quickly, at low cost, in Flash and HTML5), thus requiring minimal investment and risk on the part of developers. While the profit per unit is low, Casual developers are promised much higher royalty rates (70\% for platforms such as Facebook, the App Store, and the Android Market), and access to a much larger "install base", and thus look to profit through an economy of scale. Perhaps most importantly, Casual game sectors seem to promise an escape from the increasing publisher oversight

142To reiterate, Casual (with a capital " $\mathrm{C}$ ") is an umbrella term referring to all casual (e.g. puzzle games like Bejewelled), social (e.g. social network games like Farmville) and mobile (e.g. iPhone games like Angry Birds) games. In this sense, Casual is opposed to Core, which refers to console, PC and indie games. 
that characterizes console development.

Academics have just started to chronicle the ascendance of Casual games (Bogost 2010; Deterding 2010b; Whitson 2011; Whitson and Dormann 2011; Juul 2010; Consalvo 2009a; Begy and Consalvo 2011), most often critiquing the simplistic mechanics of social games (especially the monotonous clicking). As of yet, there is little reflection upon the underlying socio-economic conditions that have made the game industry ripe for revolutionary shifts towards Casual games, nor the new economic structures that heavily influence game design and the tensions these socio-economic shifts create within the development community. Most discussions of Casual games and digital games in general are focused on players' interactions with the game, and players' interactions with each other. Questions of how these games are made, who makes these games and why they are made in the ways they are, are alluded to, but left unanswered. Idealistically, we could answer these questions using notions of artistic creativity on the part of designers and developers fuelling the paidiac experience of players (i.e. developers are shifting to Casual games as they offer more opportunities for creativity, exploration, and experimentation with play styles). However, these questions are more realistically answered by first attending to the socio-economics of game production. The following examination of social games provides an ideal entry point into examining the larger economic shifts in game production.

The recent growth of Casual games is rooted in social networking services and the popularity of social games. Social games have traditionally been defined as games in which the "play consists primarily of social interaction between participants" (Salen and 
Zimmerman 2004:622). Historically, all games were social in that they were played with other people. It is only recently that single player games emerged and become the norm, at least in terms of computer games (Pearce and Artemesia 2009). But this definition is not what is meant when publishers and developers refer to social games. In fact, they are referring to something very different. The term "social games", at least in the game industry, now refers to games that are accessed and played within social networking sites such as Facebook and MySpace. In this context, "social games" are shorthand for "games played using social networking platforms". Accordingly, I wish to emphasize that unlike the traditional game studies meaning of the term, with this new common usage term, there is nothing inherently social about social games (Deterding 2010b).

Aki Järvinen, developer, author and academic, defines social games as "online games that adapt the players' friendship ties for play purposes, while accommodating their daily routines" (2010). Writer and game designer Sande Chen (2009) lists a few specific traits that may define social games beyond their existence on social networking platforms and smart phones. These traits include:

- Multiplayer games that utilize the social graph, i.e. a player's social connections, as part of the game. e.g. Parking Wars.

- Games in which the main game play involves socializing or social activities like chatting, trading, or flirting. e.g. Pet Society

- Turn-based games that are played within a social context or with friends. e.g Words with Friends

- Competitive casual games that include friends-only leader-boards e.g. Who Has the Biggest Brain?

Social games harness pre-existing social networks to market and disseminate the game amongst communities of players. Embedding these games within players' social networks of friends, family, and co-workers motivates play. 
Social games are extensions of traditional casual games. Players are drawn to social games for the same reasons they are drawn to casual games. Their design exemplifies Juul's five traits of positive fictions, usability, interruptibility, lower difficulty and punishment, and juicy feedback (Juul 2010). But the deep allure of social games is rooted in the interaction with other players. According to Juul, social game design "isn't about creating a game that is strategically deep as much as it is about making sure the game, in turn, creates interesting interaction between players" (2010:122). Social games leverage the successful techniques of casual games and build upon them by incorporating one's real life social ties into game play.

While social games are not restricted to Facebook, the examples used in this chapter are primarily Facebook games. This is because the widely publicized success of Facebook games has propelled the rise of Casual games on other platforms (especially mobile platforms such as the iPhone), attracted developers to Casual sectors, and is setting the standards for the emergent Casual games industry in North America and Europe. Simply put, early successful Facebook games such as MafiaWars and FarmVille have set precedents for how all Casual games are now made. ${ }^{143}$ For example, in the fall of 2011 Zynga, the publisher of games such as FarmVille and MafiaWars, was evaluated at between $\$ 10$ billion and $\$ 20$ billion (Orland 2011d). This easily surpasses the NASDAQ value of console publishing giant EA, whose own recent rise in stock valuation was due to the purchase of Angry Birds creator, Chillingo (Meer 2010b). With over 800 million members, Facebook has introduced gaming to a massive globally distributed audience

143 It is important to note that while Facebook is currently the most popular social networking site in North America, this is not the case in much of Asia. Accordingly, the influence of Facebook in terms of design is exceptionally strong in North America and Europe, but varies elsewhere. 
(Olivarez-Giles 2011). Social games attract diverse audiences in comparison to Core games - over $60 \%$ of those who play the top Facebook games are female, and players over the age of 35 are a quickly growing demographic (S. Su 2010). This diverse and widespread audience made Facebook a compelling platform for developers, and the demographic and financial growth experienced by key Facebook developers has become a model for developers on platforms other than Facebook, especially in the mobile sector.

Casual game design strategies and techniques originating on Facebook have consequently migrated to other platforms (e.g. iPhone Apps) and entire games such as FarmVille have been ported to numerous other sites. Zynga has become the "Goliath" of the Casual games industry and has cornered the Facebook market (Caoili 2010a; Siegel 2010). While this leaves room on Facebook for smaller developers to cater to niche markets (Morrison 2010a, 2010b; Siegel 2010), larger developers are focusing on other markets and platforms, particularly mobile and app games, which incorporate all the affordances of social games as well as the benefit of being accessible by the player at all times via their smart phones (Meer 2010a). Accordingly, although Facebook has certain technical affordances and traits that may differ from other social networking platforms, I am using Facebook games in this chapter as exemplars for the much larger category of Casual games.

While the casual game sector has positively influenced the growth of the social games sector, there is an elision of 'casual games' and 'social games' in the game development industry. 'Social games’ are subsuming and becoming representative of all Casual games (or at least what Casual games 'should be'). In fact, most social games are 
casual games, but industry attention is centred on social games, leaving the category of casual games at the wayside, at least in terms of developer publications and conferences. There is a subtext here that Casual games are better if they are social. This subtext is directed not just at Casual games, but all online games, including Core. Making a game 'social', in this context, translates to linking the game to social networking platforms. By extension, the revenue-generating structures, online distribution channels, and datamining metrics of social games are depicted as the forward-thinking improvements on the traditional console retail model.

\subsection{Funding the Casual Revolution: The economics of development}

While game developers and publishers have historically concentrated on creating AAA Core titles, many are shifting their allegiance to Casual game development. Some have entirely moved away from the console and PC sectors to social game start-ups, while others are diversifying and adding Casual games (both browser and mobile based) as a supplement to their Core development. This transfer of loyalty can be explained largely in terms of the economics of development and the underlying tensions and constraints this system creates between publishers and developers. Much of this tension is rooted in the leading role that publishers play in the development of console games.

\subsubsection{Core Development}

To reiterate points made in Chapter Six, Core game developers only make money once their games reach retail markets. They depend on the financial support of publishers to 
support multi-year production schedules, buy hardware and software necessary to produce 'next generation' games, and to pay licensing fees and employee salaries (Kline et al. 2003). Besides providing operational funds, publishers are essential in negotiating retail exposure and getting games onto big box store shelves. Despite the growth of digital distribution detailed in Chapter Eight, big box stores such as Wal-Mart and Futureshop still account for the majority of game software and hardware sales (Crossley 2011b). Publishers are the only source of funding for most developers unless a prior game is earning royalties, as venture capitalists are loathe to finance game development studios (Lovell 2010). Beyond financing, publishers are also responsible for quality assurance testing, manufacturing, marketing, and distribution once the game is developed.

Development costs, timelines, and the size of development teams increase with each console generation. According to veteran game developer Brian Fargo: ...10 years ago, you could make retail games for $\$ 2$ or $\$ 3$ million. Four million dollars wasn't a big deal. Now with these goods, these things can [cost] \$100 million. It's made publishers crazy. Whole careers are on the line. Whole companies are on the line. Creating products creates this intense pressure. (as quoted by Graft 2010)

The chances for publishers to recoup every advance are slim because sales are concentrated on a small number of hit titles. In terms of sales revenue, the top twenty games "generate $80 \%$ of the industry revenues while hundreds of other titles make up the remaining 20\%" (Fullerton 2008:423). And, while the cost of development steadily increases, retail prices of games have remained relatively stable, thus contributing to declining profit margins (Fullerton 2008). Not every game 'breaks even'. In these cases publishers fail to recoup their initial advance to developers. 
Accordingly, publisher support comes at a substantial cost to developers (Carmel 2010). Because publishers assume most of the financial risk, they claim most of the profits (if there are any), as well as the intellectual property rights for the game. In order to ensure a game's success and to manage their initial investment, publishers are now inextricably involved in directing game development. Publishers wield considerable power over development - not only stipulating scheduling timelines and milestones the development team must meet, but also having a say in the design of the game and the creative process (Carmel 2010). This hierarchical chain of production results in constraints that hamper the creative process of development and compel a bureaucratic ethos fixated on milestones and certification tests rather than the creative autonomy that many developers desire. Acclaimed designer Tim Schafer explains :

With a triple-A game, when there's so much money invested, the risks for a publisher are huge. The more money you ask for from an investor, the more that you have to give up. No matter where in the world your publisher is based, they will remove features that could potentially alienate any users when the stakes are so high. (as quoted by Parkin 2010b)

The tensions created by this publisher/developer relationship are further detailed by Casey O'Donnell in his three year ethnography of American and Indian game development studios: "As far as developers are concerned, publishers and manufacturers control the video game industry. Development studios structure themselves around their relationships with these companies" (O’Donnell 2008:145).

This financing structure pits developers against publishers. In securing publisher funds to develop their game, developers sign away their IP rights as well as most of the royalties the game may earn. Thus, developer revenue streams are relatively static and 
may not reflect whether a game is sold at a loss or whether it is a commercial success.

The publisher advance is likely the only profit developers will see from the game.

Accordingly, when negotiating this amount, developers may 'mark-up' the advance,

including leeway for new features, changes and revisions demanded by the publisher,

cost overruns, and a margin for profit. By inflating this profit margin, developers can

finance the initial development of their next project, thus allowing them to escape future

publisher oversight (at least in the initial stages). For example, for a $\$ 10$ million dollar

advance, developers would initially budget $\$ 6$ million for development costs (Benton

2010). Brian Fargo explains this relationship:

The risk... I mean, the bigger risk, frankly, is on the publisher, right? I mean, these days, to put out a title, by the time they spend production, manufacturing, marketing, they're between $\$ 80$ million to $\$ 100$ million. Huge risk. So, in this particular case, I'm on the development side now. As developers doing a triple-A game, the upside is you get your costs covered. There's not a lot of money to be made as a developer getting straight-up advances. Publishers are not going to allow you to get rich off of just straight-up advances. You're having to count on the recoup. On the upside, you have your downside covered. But it's hard to recoup [on bigbudget games] because of the cost. (as quoted by Graft 2010)

Because developers sign away their IP and most royalties, there is less financial incentive for them to create the best game possible. Developer Brenda Brathwaite explains this dilemma in a recent blog post:

Several years ago, I was working for EA designing the lifestyle system for an urban fighting game. I had it FINISHED, and planned to send it off that day. Then, an idea occurred to me that would make it better - not massively, but maybe by $10 \%$. It was certainly a better way to do it, the right way, I would argue. Now, I vividly remember thinking about how much time it would take me to make this change. It was going to affect EVERYTHING I'd already written. I was on contract for a fixed fee, so it didn't matter whether my work took 200 or 300 hours. Done was done and $\$=$ the same. It was the first time I had been in such a situation. 
I vividly remember everything about that moment. Where I was sitting, what I was doing, what work the decision would affect. It wasn't a pleasant thought. But then came an important moment of professional growth for me my only responsibility was to do it right for the game. That is what I was hired to do. So, I took the massive amount of time, the stress on other things in my life (I was just starting at SCAD at the time, and that workload was nuts), and I did the job right. The critical thing is that had I not done it, NO ONE would have known. It was good enough, but I knew I could do better. (Brathwaite 2011a)

Conflicted, Brathwaite made the decision to rework the game despite the personal costs in time and labour. While many developers applaud Brathwaite's ethical decision, oftentimes development schedules are so tight that there is no room for this sort of improvement, or the extra work fails to get approval from upper management. Accordingly, despite the best efforts of individual developers, the system as a whole incentivizes making the game as cheaply and quickly as possible, as schedule extensions and iterative improvements decrease profit margins. Accordingly, traditional financing structures pit publishers and developers against each other rather than offering incentives for them to work in alignment.

Simply put, the perceived differences in constraints in Core development compared to Casual development are what fuel the shift in the larger game industry. Whereas console development is rife with hierarchies and chains of command, social development is flat, composed of small agile teams composed around game projects. Veteran developers, including both Brian Fargo and Brenda Brathwaite, have moved to Casual sectors, especially social and mobile games, to escape some overwhelming constraints of Core development.

The overall organization of the game development industry and, in particular, the 
economic constraints on console development, promote instrumental 'play' on the part of developers. Thus, game development itself, much like power gaming, has become a "soft coercive system" (Taylor 2006a). Developers freely choose to engage with the system, but their actions are undeniably constrained and oriented towards certain directions, such as unending grind and conserving resources. While resistant to change, Core developers are realizing that they are playing a losing game of their own. As reports of crunch increase (Crossley 2011c; R. Fahey 2011a; Remo 2010b; M. H. Williams 2011), and creative autonomy decreases in favour of risk management strategies imposed by publishers, Core developers are realizing that their own work has been effectively instrumentalized. Yet, as these modes of work/play become increasingly mandatory, developers start to push more and more against the boundaries of the system. The growth of gamification and Casual games, as well as digital distribution methods all evidence this pushing against the system in response to constraint, sending game developers moving in new and innovative directions.

\subsubsection{Casual Development}

Established developers of Core games who have shifted to Casual development believe that these games represent a "new frontier where radical and rapid innovation exists, in contrast to the more conservative world of AAA retail games" (S. Johnson 2010). Respected 'old school' designers like Brenda Brathwaite, Steve Meretzky, Sid Meyers, Raph Koster and Noah Falstein are all designing social games for Facebook (Hyman 2010; S. Johnson 2010). In part, this shift towards Casual games is predicated on the 
search for creativity free from publisher oversight. Returning to the theoretical underpinnings outlined at the start of this chapter, increasing constraints in Core development have brought many developers to a tipping point: publisher-imposed constraints, in particular, have limited the directions that developers can take. Publishers are risk averse, and thus restrict the content of the games they publish, favouring 'proven winners' over innovation. Frustrated with the lack of autonomy and room to innovate, developers look for ways to escape publisher oversight. Platforms such as Facebook are ideal targets for these developers.

In contrast to Core development, casual, social and mobile development offer significant benefits to both developers and publishers. They offer lower development costs, shorter development cycles, and quicker times to market. In comparison to Core games, which can take up to 4 years to develop, small flash games such as those found on Facebook can take a small team of 3 or 4 people a few short months to build. Shorter development cycles are attributable to the smaller size of these games and the simplicity of the technology. For example, the massively popular FarmVille was released after just 5 weeks of development (Kohler 2010), while Crowdstar releases both social and mobile games after development cycles of 2 - 4 months (Nutt 2010).

Publishers are lured to Casual development for a different set of reasons. Because development costs are much lower (e.g. $\$ 150,000$ for a flash game rather than $\$ 50$ million for a console title), there is less financial risk for the publisher. The accessibility and broad demographic appeal of Casual games make them particularly attractive to publishers. In order to cater to an economy of scale, Casual games are explicitly designed 
to attract large audiences. Most often downloaded online and played in web browsers, Casual games "can be played in short time bursts, and generally do not require an intimate knowledge of video game history in order to play” (Juul 2010:5). They have simple interfaces and can be picked up and played with minimal instruction and for short sessions of time. In comparison, Core games generally require some skill and foreknowledge (e.g. genre conventions such as spellcasting in RPGs, button coordination in FPS, etc.) as well as considerable time commitments. Casual games are additionally attractive to publishers because of thematic considerations. For example, Juul argues that Casual games, unlike Core games, are generally set in pleasant environments and have a positive emotional valence (Juul 2010:50). In supporting Casual games, publishers and developers thus avoid conflict with ratings boards and lobbyist groups concerned with media violence, and can market their games to a much wider audience.

Despite their differences, in each Casual sector (mobile, social, and casual), the move to digital distribution challenges the traditional publisher role. Due to lower development costs, developers turn to other financing options. And because Casual games are digitally distributed, developers are no longer reliant on publishers to manufacture and distribute the physical game, nor do they depend on publishers to negotiate retail deals in order to get their game on store shelves. Although developers may turn to publishers for their marketing acumen and knowledge on how to attract and retain customers, they are no longer dependent upon them. Traditional publishers retain influence because of their financial strength and brand power, but they are forced to innovate in order to maintain their powerful position in the game industry, "transitioning 
both organically (by getting offline products online and elongating development and marketing cycles) and inorganically (by acquiring online businesses)" (Gibson 2010:12). Publishers such as EA transition by purchasing Casual games companies, whereas others such as Ubisoft and Activision Blizzard create their own portals and link them to Facebook (Blizzard Entertainment 2010; Takahashi 2010). These efforts evidence the changing relationship between publishers and developers, and how Casual development alters their traditional roles.

Changes in the game industry are not all due to developer-publisher relations. The shift away from Core development also stems from developers' own lives as well as business imperatives. For example, Brian Reynolds ${ }^{144}$ states that the impetus to move from Core to Casual design is rooted in changes to his own play habits - he wants to develop games that fit with his current play style (Alexander 2010c). Older, with families and other responsibilities, many developers have less time for time consuming Core titles, and thus prefer games that easily fit within their busy schedules, and/or that can be played with their children and families. Moreover, after working with development teams of unwieldy size (ranging from 30 - 200 people for console and PC titles), Casual games offer a return to the 'garage game' ethos wherein games potentially can be made by a few people working together in close concert (although given the growing emphases on metrics, monitoring players, and constantly updating Casual games, this may be difficult to do in actual practice without the assistance of marketing and business specialists). For some developers, Casual games hold a nostalgic appeal and are a welcome return to

144 Reynolds was lead designer for Civilization II and co-founder of Firaxis Games and Big Huge Games (both Core strategy game companies), before he became the Chief Game Designer at Zynga. 
shorter arcade-style games and a respite from the exhausting and time consuming production of Core titles. Fargo further argues that:

$[W]$ ith the smaller stuff, you get kind a return to the roots. One of the things I do like is that part of the industry reminds me of the late ' $80 \mathrm{~s} \mathrm{-} \mathrm{Small,} \mathrm{creative}$ teams trying to figure out how to make a buck. You didn't have these huge costs and risks. The costs and risks turn down because you're kind of able to creatively try some different things. (as quoted by Graft 2010)

Clearly, there are many facets of Casual games that make them an appealing opportunity for developers.

However, developers may not find the freedom they desire in the shift towards social games. At the time that Aphra Kerr was writing The Business and Culture of Digital Games (2006), an earlier flight from publisher oversight stimulated the casual games industry. Many developers migrating from Core to casual development discovered that they merely exchanged one set of overseers for another, as Margaret Wallace discusses:

There is a vicious cycle where the people with access to the audience are making demands on the kinds of content being made, not wanting to take a lot of risk due to the quarterly numbers, but at the same time developers don't want to take risks because they want to appear on the portals. (as quoted by Juul 2010:212)

While social networking sites, portals, and the App Store do not have the same content restraints of publishers, developers must still acquiesce to the demands made by platform owners or risk having their game suspended. Platform holders thus maintain their gatekeeping role, even with digitally distributed games. As one developer puts it during his launch announcement of an indie-run digital distribution platform:

The availability of games in online stores is dictated by relationships with publishers that the platform-holder needs to nurture. It doesn't matter how 
good your indie game is, if it conflicts with [INSERT BIG PUBLISHER HERE]'s next AAA title then you're not getting green-lit. If giving users a decent search/recommendations feature reduces the platform-holder's leverage over their heavyweight content-providers, then it just isn't going to happen. In the case of mobile app stores, either the owners don't care or they're too busy rolling around in money to care. It works well enough for them, so who cares? (Deejay 2011)

Instead of bottle-necking physical production, platform operators have taken on a "digital curator" role, as evidenced by Apple's closed iOS App Store, and XBox Live's limited slate of games.

As Casual platforms become more popular, platform holders demand a larger percentage of the profits and more control over content. ${ }^{145}$ Favourable profit shares attract developers to new sectors early in the platform cycle. As the platform matures, amassing both its player base and stable of developers, the profit share ratio increasingly favours the platform holder. And as Casual game sectors become increasingly competitive, developers are compelled to further divide their profits, enlisting the aid of publishers who will ensure visibility in a crowded marketplace, as well as other service providers that aid in marketing and advertising (and selling advertising space), as well as enlisting monetization services that ensure smooth financial transactions and payment security.

However, despite the increasing similarity to the Console space, the Casual Revolution and the growing popularity of casual, social and mobile games makes sense. It provides accessible games to a broader demographic of players. It decreases the cost

145For example, before 2011, developers of social games on Facebook kept all profits for the game. This profit divide was vastly preferable to the $40 \%$ share for physical Console games (which in turn was drastically reduced by the need to divide this $40 \%$ with the publisher). However, as the sector grew and greater numbers of developers flocked to social networking platforms, the profit share decreased due to the 30\% microtransaction tax imposed by Facebook. Facebook developers complied with other substantial restrictions, including the limiting of notifications and the prohibition of any currency other than Facebook credits, because they are loathe to abandon the captive audience of 800 million Facebook members. 
and time commitment of development, allowing developers to build games more quickly. Publishers benefit by producing these games at lower cost, marketing them to a wider audience, and reducing their own economic risk. Because digital distribution alleviates developer dependence on publishers, publishers are also pressured to innovate in order to maintain their market share. This revolution is impacting the shape of the industry as a whole, and influencing what types of games are made: Casual game development is exploding, and it is becoming increasingly difficult both to fund Core game development and to profit from the games themselves (Parkin 2010a). While the revolution started first in casual games, it is social games and their economic structure that fuelled the flames, and mobile games that are currently setting the industry ablaze. Simply put, Casual games are inexpensive and quick to create compared to console games, yet social games have proven that the revenues of Casual games can match that of console games. Consequently, the revenue structures of social games are influencing changes in the revenue structures of Core development, as well as the other sectors of Casual development.

\subsubsection{The Particular Economics of Social Games}

Traditionally, players spend money to play digital games. They feed quarters to an arcade machine, purchase hard copies at retail stores, or buy monthly MMO subscriptions online. But tradition is changing, reshaping the economics of games and, consequently, altering how games are designed. Social games are free-to-play (F2P), meaning that players can access and play the game without buying it outright. Not restricted to social 
games, the F2P model, as we saw in Chapter Eight, is also common in online MMOs, casual games, and app games. According to Neil Young, ex-EA executive and founder of iPhone games company, ngmoco, the growth of F2P games is "the most significant shift and opportunity for [game developers] since the birth of this business" (Young 2010). As stated by Brian Fargo, "It changes the business model, and people are now making money from advertising or selling goods on a free-to-play basis. And the people who are playing these things are doing it in sort of bite-sized chunks... And the good news is you can jump in and experiment without spending a ton of money" (as quoted by Graft 2010) F2P game design is tangibly linked to business success in the form of monetization. Revenue streams for social games are threefold : 1) microtransactions (i.e. revenue from players themselves); 2) advertising; and, 3) lead generation. Monetization, in turn, is intimately tied to metrics, the collection of player's data and their interactions with the game. The player's desire to advance quickly in a game undergirds microtransactions. In short, microtransactions allow players to purchase in-game currency and virtual goods so they can level up faster, customize their play, and improve their overall experience. In most F2P games, every action requires energy and energy is finite. This energy is replenished or earned on a regular basis, but this is not frequent enough for motivated players. Thus motivated players purchase a master currency that can be exchanged for elements that effect game play For example, Frontierville's master currency is the horseshoe which can be used to purchase energy, but is also used to attain reputation and social capital - by buying 'rare' items such as animals, decorations, and buildings, or giving extravagant gifts to one's friends. Typical microtransactions are 
generally under $\$ 2$, a low cost suited to impulse buys on the part of the player. While often critiqued as a nefarious scheme to force users to pay in order to access vital parts of the game, this minor expenditure can be easily rationalized by players who otherwise may be spending $\$ 70$ on an untested retail game, or even $\$ 15$ on a monthly MMO subscription (Schell 2010b). Only a fraction of players use microtransactions - an average of $1-2 \%$ for most games, and up to $10 \%$ for the most economically successful games (Young 2010).

Advertising is another avenue for social game revenue. Social games generate advertising revenue by placing third-party interstitial ads, banner ads, video ads, links, or branded virtual items inside the game. Companies such as AppSavvy, LivingSocial.com, and Cubics broker agreements between interested advertisers and social games publishers. Alternatively, developer/publishers can sell ad space independently, or crossmarket other games in their line-up. Because social games are so thoroughly imbricated in players' social networks, they are an ideal site for advertisers who can selectively target their ads based on the player's listed gender, birth date, geographical location, media preferences, and even their political and religious affiliations - information that is available to social game developers once players install the game application (although player's privacy settings may restrict the extent of this information as well as how long developers have access to it). As a side note, players are often unaware at the depth of information available to third party game providers, and do not realize that these developers and affiliated advertisers may not be bound by the privacy contract between Facebook and its users. Accordingly, players' personal information is exposed to the 
prying eyes of advertisers, who pay game developers and publishers for access to lucrative demographic information as well as data on how players respond to their ads (for example, which demographic of players is more likely to click on the ad).

In terms of lead generation, publishers turn to companies like TrialPay, Super Rewards and Offerpal Media. Simply put, lead generation is a referral fee system. In exchange for filling out surveys or offers to join NetFlix, Blockbuster, apply for a credit cards etc., players receive virtual currency or goods. Companies such as NetFlix then pay the publisher for each referral, using Super Rewards and Offerpal as a middleman. Other lead generation offers ask users to install other games as a way to earn credits for the game they are currently playing. According to Young (2010), 18-32\% of users download and install these offers in exchange for some virtual good. Lead generation results in more revenues than microtransactions themselves (Schell 2010b; Sheffield 2010). Lead generation, while a profitable revenue stream across many social gaming platforms, raised considerable controversy over offers that automatically signed players up for recurring fees, unwanted products, and cell phone charges (Arrington 2009; Luscombe 2009). As reported by Newsweek, Facebook, in particular, was slow to police these scams partly because these leads generated substantial advertising revenues for Facebook itself (Lyons 2009). This unwanted media attention tainted lead generation techniques and thus put their continued use into question. However, lead generation companies such as Applifer.com take a less controversial approach, helping game developers set up crossreferral services to attract players to their game in exchange for promoting other developers' games to their players. ${ }^{146}$ 146Applifier, in turn for this free service, is allowed to insert paid game ads into participants' games. 
The financial model of F2P is predicated on close communication with players. But this communication is not social (in the traditional sense of the word) but is rather premised on the collection of player data. This data tells developers who is playing their game, how, for how long, and, importantly, how much they are spending. For social games in particular, communication is a numbers game rather than a language game: developers and publishers rely more on statistics than fan forums or critical feedback to drive their design decisions. The financial model of F2P games is also undergirded by highly developed surveillance structures. Unlike retail games where the money is collected up front in one lump sum payment, F2P games are predicated upon maintaining players over a long period of time and monitoring their behaviours. Games are launched early as MPVs (minimum viable product) and are purpose-built to mine real-time user data (Young 2010). Data collection is widespread, including the age and gender of players, how long they play and how often, their friends lists and transactions history, and even - for iOS apps - where they touch their screens.

This data is commonly used to make iterative improvements to the game. This allows game designers to test different features and gather detailed feedback on player interactions, and is one of the factors that set social games apart from traditional Core games. For example, Brian Reynolds, lead designer at Zynga divided Mafia Wars players into different groups in order to test the accessibility of seven different tutorials running in parallel: "We learned all kinds of stuff about what it takes to get somebody to become a regular player...We went down to three, then one, and that one was totally counterintuitive to what we thought the tutorial should be like" (as cited by Elliott 2010). In 
response to these metrics, F2P games are remotely adjusted 'on the fly' in order to optimize three main things: monetization, retention of users, and reach (a measure of how many players have been exposed to the game) (Young 2010). This continual surveillance opens up new revenue streams, as data can be sold to advertisers as well as used to attract new investors.

Metrics and the resultant design decisions are deeply tied to economic concerns. Individual player data is aggregated and compiled into business-centred metrics such as DAUs (daily average uniques) and ARPUs (average revenue per user) that measure how lucrative these monetization measures are in the short term (i.e. how much money is spent per user per day) and in the long term (i.e. predicting the monthly growth in game revenues and valuating the market price of the game development company). Changes in the structure of game design are monitored to see how they impact DAU and \$/DAU (the average amount each unique spends on a daily basis). \$/DAU is a measure of how well the game is "monetizing usage", and, at least in the eyes of proponents of F2P games as well as publishers and venture capitalists, is the most important measure of how successful a game is. Monetization is the "fuel that drives [the] thinking" of the F2P game industry (Young 2010).

While the average user may only spend a few pennies on a F2P social game, the potential revenue of these games balloons in economies of scale. FarmVille at its peak claimed 33 million DAU compared to World of Warcraft's 11.5 million monthly subscriptions (Bethke et al. 2010). World of Warcraft costs around $\$ 150$ (for the original game and expansion packs), plus an additional \$15 monthly subscription fee. For 
economic as well as demographic reasons, the fact that FarmVille's player population trounces WoW's is not surprising. What is surprising is how lucrative these free-to-play games can be. Zynga’s revenue for 2010 was $\$ 597.5$ million, while documents from its filing for IPO led the New York Times to speculate that the company was worth as much as $\$ 20$ billion USD (Rusli 2011a), nearly double its estimated value from February 2011 (Rusli 2011b). In the summer of 2011, Zynga made an average of 5 cents per day from each of its 59 million daily users (Orland 2011d). While this seems like a paltry sum compared to console game prices of $\$ 70,59$ million DAU x 5 cents $=$ revenues of $\$ 2.95$ million each day.

The MVP model means that social games can be developed and launched quickly (within a few months) at low cost (with budgets in the tens of thousands of dollars, rather than in the millions). In comparison to retail games, whose main revenue is concentrated in the short time period the game is on retail shelves (generally 6 weeks), the revenue stream of social games, as well as MMOs and other subscription based games, extends for a much longer period of time. This revenue stream is also more predictable thus making it an attractive investment for publishers and the venture capitalists who would normally avoid funding game development. The economic model of social games has distinct affordances for developers and publishers. Coupled with the wider demographic reach of platforms such as Facebook, it is not surprising that the game development industry is flocking towards social games. Yet, the revolution is not without its problems. 


\subsection{Opposing the Revolution: Critiques of social games}

Given that social games are disseminated through digital distribution methods and can be marketed through viral channels, manufacturing, advertising and distribution costs are reduced, leaving more room for potential profit. In terms of economics, the shifting allegiances of publishers and developers are understandable. Yet, the rise of social games is not unproblematic. Incorporating the 'social' into games is done in a manner that emphasizes bureaucratic measures like retention rates, monetization levels and metric analysis rather than meaningful levels of engagement. Many developers feel that the design of social games is rooted in business, subordinating the practice of making games to the practice of making money. Social games create a rift in the game development community. These rifts are not uncommon (for example, the divisions between Core vs

Casual, console vs PC, Sony vs Microsoft). Described in terms of a war between 'business' and 'creativity', there is an emerging division between "persuaders (developers who want players' money) and the rest of us (who want to give the players joy)" (S. Johnson 2010). This view is summarized by game designer and writer, Greg Costikyan (2010):

1. Social network games produce easily trackable metrics.

2. It is thus possible to expose a portion of your audience to a new feature and determine, with no possibility of argument, whether or not that new feature increases your revenues.

3. It is, however, impossible to use the same metrics to determine whether or not, say, your players now feel they are having more or less fun, or whether they feel they are having a more or less emotionally impactful experience, or, indeed, to track any aesthetically meaningful criteria.

4. Consequently, the design of social network games is driven entirely by business rather than aesthetic criteria.

Social games have a paradoxical effect: increasing economic constraints in the Core 
sector limit developers' creativity. The social sector attracts developers, offering a much different economic structure (F2P over retail) thus lessening the sometimes stifling hold of publishers. Yet, the F2P model propagates creative constraints due to its current reliance on metrics-based design.

Following this argument, instead of creative artistry and design, social game development is largely influenced by marketing and behavioural science. Critics argue that social game design is premised on "psychological tricks" such as variable reward schedules (Schell 2010b) rather than a creative craft. Chris Hecker, game developer and consultant, sees this as a divide between intrinsic motivators (e.g. playing a game because it is fun, engaging, and immersive) and extrinsic motivators (e.g. playing a game for social standing, points, leaderboard achievements and trophies) (Hecker 2010). Hecker's theory extrapolates from the well-known psychological tenet: adding extrinsic motivators to a task results in declining intrinsic motivation. In other words, players are willing to engage in repetitive dull tasks (e.g. clicking monotonously to plant, plough, and harvest virtual crops in games like FarmVille and FrontierVille) if they are extrinsically rewarded, even if their appreciation for the play itself is diminished or was non-existent to begin with. Social games players continue with these never-ending tasks because their friends are also continuing with the same monotonous grind. ${ }^{147}$

Critics perhaps unfairly single out the most popular Facebook games for their critiques, and hold them representative of all social games. These games are shamelessly derivative. They are 'reskinned' clones of clones and outright copies of other developers' art, concepts, and mechanics. Most of Zynga's games (if not all) are direct reproductions: 147Yes, this parallels the critique of power gamers and instrumental play from Chapter Four. 
FarmVille is a reproduction of Farm Town developed by Slashkey, whereas Mafia Wars is a reproduction of Mob Wars developed by David Maestri. Similar in name, game play and graphical style, these games are blatant clones that have successfully 'built a better mousetrap', offering slight improvements on the original games, aggressively advertising them, and thus attracting much larger player populations.

While nothing directly inhibits innovation and creativity in social games, in an economy of scale such as Facebook and in Casual games more generally, the games that profit the most are ones that attract players. Only $1-2 \%$ of players actually purchase anything, and even then transactions are generally under a dollar or two. Accordingly, in order to offset operating costs along with the continual costs of updates and new content, social games must attract larger audiences. ${ }^{148}$ Larger audiences also attract lucrative advertising and lead generation partnerships. The games that are simple to play, thematically benign, and cater to the largest denominator are the ones that are financially successful. This, in turn, promotes the inclusion of viral mechanics and 'spam' notifications as developers must continually recruit new players in order to retain a large player base. While this can be achieved through aggressive marketing, to reduce advertising costs or avoid them altogether, developers utilize viral mechanics that mine existing players' social contacts, and offer incentives for them to play the game.

Ultimately their success in attracting players, as well as the funding of investors, means that these heavily critiqued games are held as ideal models of social games.

148 The alternative is catering to a devoted niche audience that is willing to pay more than a small ( $\$ 1-\$ 2)$ microtransaction amount. But with higher expenditures, players expect more content and increased game complexity, both of which cost money and time to develop, and may not be technologically feasible on social network platforms such as Facebook that only support flash. This can lead to a tradeoff: to offer a technologically complex game to players, developers must move away from platforms such as Facebook. But in doing so, they lose access to the massive user-base that the platform offers. 
Ian Bogost articulates a four-part critique of social games. The first part of his critique is rooted in the Heideggerian notion of enframing. The design of social games enforces the belief that "people are just the things they might do for you when you need them" (Bogost 2010). Games such as FarmVille simplify social relationships into preformatted notification messages and structured visits to neighbours' 'farms', instrumentalizing social ties and negating the need for any deeper social interaction. Bogost explains: "In social games, friends aren't really friends; they are mere resources. And not just resources for the player, but also for the game developer, who relies on insipid, "viral" aspects of a design to make a system replicate" (Bogost 2010).

The second part of Bogost's argument echoes Hecker, emphasizing the psychological manipulations and exploits utilized by social game developers to compel players to continue playing the game. In Bogost's words:

I have a liberal sense of what a game is...I do think, though, that the kind of experiences that they're creating are more like [Skinner] boxes, like behaviourist experiments with rats. They're relying on creating these compulsions so people will want to come back and click on the bar. And so, in that respect, I fear those kinds of products. (as cited by Terdiman 2010)

While MMOs such as World of Warcraft also employ similar compulsion loops and "brain hacks that exploit human psychology in order to make money" (Jesper Juul as quoted by Bogost 2010), Bogost's argument here is that MMOs, unlike social games, offer more than simple brain hacks. In other words, there is still a place for paidia and creativity in MMOs, unlike social games. Social games have become so instrumentalized, they fall off the paidia/ludus scale entirely. They are no longer playful, and thus are not games. ${ }^{149}$

149 We can see direct ties here with the fourth transformation in the Theory of Ludification detailed in 
Bogost's third criticism is directed at the "optionalism" of the game, wherein players can skip the actual game play by delegating the tasks or spending money to overcome them. For Bogost, "social games are games you don't have to play" (Bogost 2010). The last element of Bogost's argument focuses on time lost while playing social games. While many games, especially online MMOs, demand intense time commitments on behalf of the player, social games foster a deeper compulsion that lays claim to the time we spend away from them. ${ }^{150}$ "Social games so covet our time that they abuse us while we are away from them, through obligation, worry, and dread over missed opportunities" (Bogost 2010). The timer-based operations and notification messages constantly remind players what they have to lose by not periodically checking into the game.

Bogost and Hecker liken the design of social games to slot machines. They are both driven by monetization, premised on easy-to-learn mechanics, and focused on getting users to return again and again. Yet, this drive for monetization is not a new occurrence for the video game industry, as the 'insert coin to continue' model shaped the design of arcade games. Arcade games are expressly designed to be difficult, with no pause buttons, save features, or unlimited lives. The games are nasty, brutish and short, thus compelling the player to insert another quarter to continue (Montfort and Bogost 2009:112). In the console market, monetization efforts have been off-loaded onto

Chapter Four. The difference here is that instead of making everyday tasks more like play, social games become so rationalized that they are no longer seen as games.

150To understand the allure of social games, for 75 consecutive days during the summer of 2010, I played FrontierVille on an alternate Facebook account. I can personally attest to the compulsions the game created. On an average working day, I spent over two hours periodically checking into the game to harvest crops and visit friends' farms (strangers I had 'friended' in order to progress in the game). I had to quit so that I could start writing my dissertation. 
publishers, ideally leaving developers to create the game and publishers to find a way to make it profitable, thus creating a separation between the creativity of game development and the business of game publishing. Of course, increasing publisher involvement in game development (e.g. certification, milestones, and demands for specific content) evidences that drives for monetization are not absent from the Core development process, and indicates that perhaps, in a profit-seeking industry, creative freedom is more fantasy than actual reality.

\subsection{Responding to Criticisms}

Despite these numerous criticisms, Casual sectors adapt to critique, just as we saw how Core games were adapting in Chapter Eight. Casual games take from and learn from Core games, just Core sectors learn from and are reshaped by Casual sectors. Developers migrate to the social sector and try to improve social games using techniques and mechanics developed in Core sectors (this can be clearly evidenced in the increasing complexity from early versions of FarmVille, to FrontierVille and, now in CityVille). Conversely, Core developers adapt and port Casual techniques such as metrics, viral spread, and shorter game play sessions from the social sector to other sectors, including mobile, PC, and console. Core sectors, in exchange, offer ideas on how to encourage user creativity (e.g. content creation, modding, fan forums) as well as techniques to gradually layer in complexity, allowing Casual games to incrementally develop the skill-sets of their players and offering them a more multi-faceted and rich playing experience than is currently available. Moreover, social games take lessons from online PC games, 
especially MMOs, on how to manage and nurture deep and meaningful social interaction (e.g. competition, guilds, and community formation). While the most popular social games (e.g. FarmVille, Pet Society, Mafia Wars) are often equated with the whole of Casual game development, it is important to note, their current ascendency will end, making way for more varied and sophisticated game play experiences. As argued by Brian Fargo ${ }^{151}$, while social games may have a current affinity for 'Skinner boxes' (named after the research of B.F. Skinner), gamers will demand more complexity:

So, the truth is, people, as usual, they're going to demand more over time. These little applications that people are getting away with that are pretty superficial and more like the rat and the pedal, that's all fine and dandy. What it's done is created a new kind of way of playing. People will start to demand more, more and more, and pretty soon, I think you will start to see the marrying of more traditional game elements for the purists with that sort of mechanism. (as quoted by Graft 2010)

This exchange from Core to Casual goes in the other direction as well.

There are four pillars of social games that are fruitful avenues for all developers (S. Johnson 2010). The first of which is a "true friends list", where online games can be exclusively played with one's real world friends. Multiplayer Core games, including console games and online MMOs often attempt to match players with their real world friends, although their efforts are less successful when friends have different skill levels. Secondly, social games are premised on persistent, asynchronous play. Since it is difficult for many players to coordinate in order to play with their friends, asynchronous games allow gamers to play at their own pace, and on their own schedule. Whereas most multiplayer games are predicated upon the players playing simultaneously, social games

151Another veteran developer, Fargo was the founder of Core RPG developer/publisher, Interplay. In the early 2000 's he went on to found developer/publisher, InXile, whose slate also includes successful Casual iPhone and flash games. 
allow players to $\log$ in at their convenience, play for a few minutes and then log out. Interaction with friends is indirect, and is mediated through written messages, gift giving, and requests for favours or aide on missions. Asynchronous play allows friends with conflicting schedules (or time zones) to feel like they are playing together.

In addition, social and mobile games successfully leverage the F2P business model, and so far have proven to be profitable for developers like Zynga. While this F2P model has flourished in the Asian MMO scene, it had not yet colonized Western Core sectors, until social games popularized the model. This F2P model is now leveraged by Core developers to attract the investment funds and venture capital that are absent from the traditional retail model, and relieve some of the financial pressure on Core developers. Finally, metrics-based iteration can be a positive step forward in game design because, "Retail games are developed in a vacuum, with designers working by gut instinct. Further, games get only one launch, a single chance to succeed. Most developers would love, instead, to iterate quickly on genuine, live feedback" (S. Johnson 2010). While a focus on metrics is not without numerous problems, the increased ability to learn how players interact with the game, and the ability to change the game in accordance with this data can be used to increase flexibility in design and promote developer creativity and experimentation.

As the technology of social networking platforms becomes more sophisticated, opportunities for more complex game play and social interaction within the games itself will inevitably arise. Yet, developers as well as game studies scholars must be wary of the path dependencies created by social games such as FarmVille, as these precedents may 
prove resistant to change. The heavy reliance on metrics is one such path dependency that requires further examination. In particular, the ideal of metrics-driven design carries problematic assumptions of how games should be developed that will be explained more fully in the following chapter. Metrics are focused on the quantifiable indicators of success: the number of players and the amount of money they spend. These indicators are then analyzed to determine how to make the game more "addictive": how to attract more players to the game, how to get existing players returning on a regular basis, and how to get these players to spend money (Siegel 2009). The reliance on quantifiable data means that qualitative measures of success such as aesthetics, narrative complexity, affective impact (i.e. the emotions the game inspires), and paidia, creativity and exploration, are thus de-emphasized as drivers of design.

The agency of designers is reduced, as their actions are increasingly directed by metrics. Even simple design decisions such as the colour of a button are not left to designer discretion, but rather quantitatively tested for their efficacy in retaining players and increasing monetization (Elliott 2010). Moreover, the never-ending nature of these games means that developers are tied to maintaining them as long as they are profitable. As argued by Bogost, 'Developers are told to 'listen to their players' and to enact quantitative design regimens to insure that players get exactly what they want - even if they do not know they want it. Just like playing one, running a game as a service is a prison one may never escape" (Bogost 2010).

On the part of the player, the reliance on metrics has its own set of repercussions. The intimate surveillance required by metrics may not be problematic in a single game 
space, but when these persistent games are linked to one's social network, legal identity, and everyday activities, the potential for the exploitation of these surveillant functions increases. More importantly, the reliance on metrics reduces the agency of players. Their input is important only as aggregated numerical data, one set of data in a faceless mass. This is a change from the individualized feedback gleaned from fan forums, and player testing.

\subsection{Criticism, Tests and the New Spirit of Capitalism}

A key element of this chapter is how the Casual games sector innovates, attempting to escape the constraints of the mainstream industry, and how these innovations are met and reshaped by critique. In terms of The New Spirit of Capitalism (2007), critique ${ }^{152}$ of capitalist systems casts a spotlight on the normative foundations on which Cités are based. Critique codifies what is not going well and searches for causes of this situation with aims to finding solutions. Critique always refers to fairness. Critics show how the world is ignoring this fairness, exposing people who have greater access to resources and thus procure unwarranted advantages, and how existing morals and norms hide these realities (exploitation, domination, etc). Critique leads to tests, as well as showing the injustices hidden in tests. Critique works to purify and tighten up the rules of tests, expose unfairness, ensure tests are legitimate, or even get rid of tests that don't align with current changes in values. ${ }^{153}$

152This directly relates to Boltanski and Chiapello's premise that key developments in society rest on the dialectic between capitalism and "critique". Capitalism keeps going, overcoming the crises it generates, by responding to "critique", stealing the thunder of its critics by answering some of their challenges, while diverting attention away from other issues that are unremedied or exacerbated.

153There are two ways to critique tests. First, Corrective criticism upholds the existing Cité but reveals how a specific test is unfair (i.e. how the powerful have gained undeserved advantage). Corrective 
The shifts in the game industry described above can be described as a series of displacements, tests and criticisms as developers search for new routes to success. These displacements take two key forms: 1) the emphasis on Casual play-styles and genres, thus aiming at populations other than the young males targeted by the console industry; and 2) alternative revenue, production and distribution models that use online connectivity and digital distribution to side-step the brick and mortar retail model. These novel innovations are tests of strength - their worth proven by their financial success and ability to reach a much wider demographic of players.

New tests can emerge from these displacements, generally in two ways: First, those enacting displacements may argue that they have contributed to the common good, and thus are entitled to specific acknowledgement (e.g. social game developers broadcast the success of viral marketing, or MMOs argue that more games should be F2P). Second, when local, individual, circumstantial displacements start to be seen by the rest of the community as something generally effective. Criticism plays a central role in determining these tests, ensuring they are fair, and granting displacements legitimacy.

Actors to whom displacements are beneficial develop, for themselves and others, a value (a "greatness") that conveys their hold on the world, and injects an autonomous moral dimension into their situation: "They turn these new tests into something that is justifiable. Along with this legitimization process, norms are established (quite often through legal rules) so as to differentiate between morally acceptable and morally

criticism works to improve fairness of test and ensure it is rooted in widely accepted conventions. In order to remain valid, the test needs to respond to the criticism or show how it is wrong. The second mode of criticism is Radical. It wants to do away with test, as the Cite it is rooted in is no longer valid. Radical criticisms work to circumvent tests and marginalise them, upholding a new Cité and providing fuel for displacements. 
unacceptable and abusive (i.e. selfish) ways of making use of new resources" (Boltanski and Chiapello 2005:173). This is achieved via criticism. ${ }^{154}$ There are two forms of criticism: social criticism that exposes inequalities, misery, and impoverishment, and artistic criticism that exposes oppression, massification of society, standardization and pervasive commodification. In the game industry, the instrumentalization of play, the blurring between work/play, and developer culture's acceptance of less than ideal working conditions, means that social criticisms become less prevalent for Core developers and artistic criticisms predominate. Artistic criticisms uphold an ideal of individual autonomy, authenticity, and creativity.

As the last section shows, tests are quickly materializing as developers contest, criticize, and adapt some of these displacements but not others. Many innovations are immediately adapted, such as social networking and true friends lists as they align with developers values about what 'good' play is, others are contested, such as the a/b testing and using metrics to drive design, while others - at least by Core developers - are rejected outright (e.g. lead generation and aggressive viral marketing). The criticisms detailed throughout this chapter are Artistic critiques, ${ }^{155}$ centred on the increased commodification of that comes with metrics-driven design, $\mathrm{a} / \mathrm{b}$ testing and new revenue models. Core developers argue that successes of Casual games are rooted in exploiting players. The

154Criticism is generally aimed at capitalism, because criticism is always rooted in the justificatory regime of Cités. No criticism means there is no need for a justification, and thus no need for a Spirit of Capitalism. Capitalism is always in need of justification. In other words, Capitalism needs to engage the people required for the production and functioning of business. To be capable of mobilizing people, the Spirit of Capitalism must incorporate a moral dimension.

155Interestingly, despite the common complaints about working conditions, very few developers argue that they have left Core development for Casual in search of shorter working hours. They argue that crunch is less prevalent, but their key justifications are all rooted in the creative aspects of their work and escaping the "pressures" of Core development. Monetary justifications - i.e. relating to financial compensation for their work are entirely absent. 
next chapter discusses the artistic criticisms of rationalization in further detail, showing why they fail to gain traction.

\subsection{Conclusion}

The perceived freedoms of Casual development, as well as the economic successes, have induced a shift in the composition of the game industry. Developers frustrated with Core development and/or facing the cuts and downsizing created by the collapse of mid-size "AA" games, look to the blue oceans of social and mobile games (Graft 2011a).

However, these new models come with constraints of their own (Cook 2011a). Early adopters such as Zynga prosper, while the rest struggle to claim territory. Success is often formulaic. And as these Casual waters attract more and more developers, they turn red as well. While proving that developers can profit with F2P, new financial models based on metrics are closely imbricated with designing for marketing and advertising needs rather than an idealized freedom to experiment with fun and social play.

By examining the economic models of Core and Casual development, this chapter highlighted how the rise of Casual games hastened industry-wide changes in how games are conceptualized, created, and distributed. Using the example of social games, and Facebook games in particular, this chapter showed how new modes of play and new spheres of game development are shaped by socio-economic factors. It examined the production cycles of Core vs Casual sectors, with a particular emphasis on the changing relationship between publishers and developers, one of the key sites of constraint and tension in Core development. I argued that the strained relations between developers, 
publishers, and retailers of mainstream games created necessary preconditions for the shift towards emerging sectors such as Casual games. Hoping to escape the constraints of the Core cluster, developers, despite their numerous critiques of social games, have flocked to their development. Moreover, buoyed by the success of social games, many developers from outside the games industry networks are now shifting towards games. This chapter explained how a shift from a product to an evolving service necessarily entails rationalization. Grimes and Feenberg argue that as games move online and become sites of commerce and profit, the paidiac aspects of play decrease, while the more ludic aspects increase to an unprecedented extent. In particular, rational properties such as reflexivity, boundedness, and rule-governedness all are intensified via their technological mediation: "The rules and parameters of the game system are programmed into the game code and become ever more tightly enforced and optimized. Play itself becomes subject to increasingly precise forms of measurement and calculation" (Grimes and Feenberg 2009:111). To extend their argument, as game development focuses on selling a online service rather than a one-off game, the work of developers becomes increasingly rationalized. In the next chapter, I will explore how the expanding use of metrics furthers this rationalization, turning game design into "design by numbers". 


\section{Game Design By Numbers}

So far in this thesis, I have linked both Core and Casual game development sectors to the New Spirit of Capitalism, arguing that the decline in Core is tied to its reliance on outmoded Industrial forms of production. In contrast, the emergence of Casual games is predicated upon more agile, networked forms of production. In order to survive, Core sectors adapt, embracing many of the displacements introduced by Casual sectors, particularly the use of metrics.

The primary data for this chapter comes from my online sources (see Appendix C), particularly the descriptions of how metrics work, the introductions and analysis of new companies that provide metrics services, and interviews with developers that detail how they apply metrics to improve game design. Developer conference sessions provided additional background on the often rocky reception of metrics-driven design.

By following the development and spread of metrics in both the Core and the Casual sectors, I highlight key differences between what capitalism looks like at the ground level of the game industry and Boltanski and Chiapello's version of the New Spirit of Capitalism. These differences are rooted in information technology - using predictive technologies to govern networks of players. Instead of visionaries guiding projects, reams of data are harnessed and used to point the way forward. Creativity is no longer rooted in individual developers, but is rather crowdsourced from massive player populations, leading to quantitative and thus 'objective' numbers that are used to drive game design. The lessons of this chapter can be applied beyond the game industry, showing how surveillance and predictive governance are utilized to revive the rationalism 
that was rejected in the 1960's and absent in earlier visions of the networked Cité.

This chapter is structured into two parts. The first part defines metrics: providing examples of how metrics are used in the game industry; tracing their evolution across multiple sectors; and, highlighting the increasing precision of metrics as both governance and marketing tools. The second half of this chapter situates the use of metrics as an adaptation of the New Spirit of Capitalism. It explores how the increasing dependence on metrics impacts both developers and players. This reliance on metrics changes developers' relationships with players, shifting from dealing with recalcitrant and unreliable forum users to addressing more tractable dividuals ${ }^{156}$ who are monitored through automated data-collection processes. While rationalized in terms of economic efficiency, this increasing dependence on metrics-driven design challenges developers' autonomy as essential creative forces behind game design, reshaping successful game design from an art to a craft molded via the scientific method. Accordingly, two prevalent themes are interwoven. The first is the increasing emphasis on quantitative data, coupled with the belief that this data will pinpoint more tractable subjects. The second theme is the increasing rationalization of game design and creative practice more generally.

\section{Part I.}

\subsection{Metrics: From Core to Casual}

As noted in the previous chapter, debates on the merits of metrics took centre stage at

156The term "dividuals" refers to the work of Giles Deleuze (1992). Instead of individuals - irreducible and with an autonomous sense of agency - the new subject of governance is instead the dividual. Dividuals are created by the data streams each individual trails behind them. Information is separated from individuals and recombined in new ways outside of their control. Such "recombinations" are based on the criteria deemed salient by those with access to the information, be they government officials or corporate marketeers. 
game developer conferences in 2010 and 2011 (Brathwaite 2011b; Caoili 2010b;

Terdiman 2010; Young 2010) and controversial articles on the evils of metrics and social games flooded game developer magazines and news sites (Adams 2010; Alexander 2010b; Birke 2011; Brightman 2011b; Elliott 2010). Despite this, there is very little detailed analysis of metrics applications in the game industry. ${ }^{157}$ Metrics are interchangeably referred to as game analytics (Medler and Magerko 2011), game telemetry (Moura, el-Nasr, and Shaw 2011), and instrumentation (J. H. Kim et al. 2008). ${ }^{158}$ Within the game industry, "metrics" is the most commonly used term. For the purposes of this paper, "metrics" refers to collecting player activities through logging user actions, thus providing a quantitative record of player-game interactions (Moura et al. 2011:11). Most often, writing on metrics focuses on analyzing players and monitoring their behaviour via logging in-game events, such as when they begin a level or when their character dies. This data is then used to determine how players interact with the game in their natural environment and is considered an improvement over other, more qualitative, evaluation methods such as self-reporting surveys or controlled play tests (Medler, John, and Lane 2011; Drachen, Canossa, and Yannakakis 2009).

Metrics are one part of the triad of user research that includes usability testing and playability testing (Arey and Swain 2008). ${ }^{159}$ The table 10.1, below, provides a brief

157There is some data on the use of metrics within closed academic settings, but this is experimental and not generalizable to the game development industry. This lacunae in industry sources is due to a number of reasons, foremost being corporate confidentiality and non-disclosure agreements, the lack of generalizability due to proprietary and purpose built systems, and the focus on virtual environments rather than games specifically (Drachen and Canossa 2009:203).

$158 \mathrm{Generally}$, the term that is used depends on the academic background of the user, the types of data collected, and how the data is used.

159Usability testing determines whether the user can operate the software, playability determines whether the user has a positive play experience, while metrics (instrumentation) uses tracking software to determine how exactly the user is playing. 
comparison between play-testing for usability and playability vs metrics. ${ }^{160}$

\begin{tabular}{|c|c|c|}
\hline $\begin{array}{l}\text { Type of } \\
\text { Data }\end{array}$ & $\begin{array}{l}\text { Play-Testing } \\
\text { qualitative, subjective }\end{array}$ & $\begin{array}{l}\text { Metrics } \\
\text { quantitative, objective }\end{array}$ \\
\hline Methods & observational, interviews, surveys, etc. & automatically collected data \\
\hline $\begin{array}{l}\text { Precision of } \\
\text { Data }\end{array}$ & $\begin{array}{l}\text { includes data on demographics, age, } \\
\text { gender of players }\end{array}$ & $\begin{array}{l}\text { player data is generally anonymous; but } \\
\text { can track a persistent player identity over } \\
\text { time }\end{array}$ \\
\hline Investment & $\begin{array}{l}\text { requires long-term investment in } \\
\text { terms of researchers to collect, code, } \\
\text { and analyze data; }\end{array}$ & $\begin{array}{l}\text { requires large initial infrastructure } \\
\text { investment in terms of data storage, } \\
\text { development of visualization tools and } \\
\text { collection processes, etc. }\end{array}$ \\
\hline Pitfalls & $\begin{array}{l}\text { - difficulty coding and generalizing } \\
\text { subjective player feedback; } \\
\text { - feedback may not mirror how player } \\
\text { actually behaves in the game; } \\
\text { - data collection is time-consuming; } \\
\text { - "lack of detailed and objective data" } \\
\text { (Drachen and Canossa 2009:204) }\end{array}$ & $\begin{array}{l}\text { data does not reflect the context behind } \\
\text { player behaviour and why it arises; } \\
\text { difficulty addressing abstract concepts, } \\
\text { such as "fun". }\end{array}$ \\
\hline Benefits & $\begin{array}{l}\text { can address context of play, } \\
\text { including player motivations, and } \\
\text { perceptions; } \\
\text { can address abstract concepts such } \\
\text { as "fun". }\end{array}$ & $\begin{array}{l}\text { ties game experience to specific game } \\
\text { design features with detailed records } \\
\text { of player-game interaction; } \\
\text { can be collected in large numbers at } \\
\text { low cost. }\end{array}$ \\
\hline
\end{tabular}

Ideally, metrics supplement other forms of user testing. However, on a limited budget, building the technical infrastructure of a telemetry system reads as a better financial investment, as automated data collection provides immediate data that is tractable to analysis without the need for subjective interpretation on the part of coders and test managers (Tychsen 2008).

160 Greenberg and Buxton (2008) posit that all usability evaluation can be considered harmful as this evaluation relies on weak scientific methods that attempt to prove an hypothesis rather than disproving it. Furthermore, usability evaluation in the early design stages may eliminate promising ideas or the development of multiple, competing ideas. 
Jeferson Valadares provides a concise summary of the promises and perils of metrics in his 2010 GDC Europe presentation. Metrics provide immediate, actionable, feedback that objectively tells developers and managers exactly which elements work, and which fail. This means:

1. Developers act on things that have metrics, because they are trackable and quantifiable. Accordingly, what gets measured gets done.

2. Good metrics drive positive behaviour, centring discussion on how to improve parts of the game that everyone can see are bad.

3. Well-chosen statistics allow for well-informed decisions about a game's user base.

4. Metrics are ideal for highly measurable environments, such as web environments. They lead to rapid results. (Caoili 2010b).

Metrics also appeal to the need for efficiency and cost reductions in the face of decreasing profit margins. With metrics, developers can "fight design bloat with data" (Kelly 2010). These statistics show how developers can streamline game design, telling developers what features players will actually use, and which features may be cut before wasting the time and money during development.

The drawbacks of metrics mirror the benefits. Ultimately, metrics act as 'blinders', focusing developers' attention on those elements that are easily measured. Anything that is not captured and tested via telemetry is effectively ignored. As iterated by Valaderes:

1. Developers act on things that have metrics because they are trackable and quantifiable. Accordingly, anything that lacks metrics gets ignored, even if it is vitally important.

2. Bad metrics drive bad behaviour in the company. For example, when companies tie incentives to metrics, employees cheat the system in order to increase their numbers.

3. If you are not tracking the 'right' metrics, you can be making decisions in the completely wrong direction.

4. Metrics are bad for noisy environments with long feedback loops.

5. Metrics can age quickly. "Three months go by and suddenly [what you're tracking is] not the thing to watch anymore," he cautions. "It's time to start working on some other thing." (Valaderes as quoted by Caoili 2010b) 
To benefit from analytics, developers must commit to continually experimenting, tweaking, and updating the game in order to recruit and retain the greatest number of players. And, following Valadares' last point, they must also continually upgrade and refine their ageing metrics. As argued by Giordano Contestabile, PopCap's senior director of mobile product and business strategy, this ties developers into a perpetual innovation loop:

That [shift to freemium] literally is changing the way games are designed, games are marketed, and it's changing the way games are maintained and operated...So we don't have, anymore, a developer that develops a game, publishes it, and then sits back and waits for the revenue. It's actually a living product that they need to update and keep alive over the months and over the years. (as quoted by Webster 2011a)

Keeping a game "alive" is a massive change from the "ship it and forget it" model of big box games. Metrics are essential for the shift to service, the economy of scale required by F2P, and the alternative revenue models provided via advertising. But this shift comes with an exhausting schedule of introducing new features on a weekly or twice weekly basis.

\subsubsection{Metrics in Core Sectors}

While rapidly disappearing as Core sectors adapt the innovations of Casual, there are key differences between how metrics have traditionally been used in Core sectors and how they are used in Casual sectors. The use of metrics in Core development falls into two main categories: metrics about players, and metrics about the development process.

Developer facing metrics concentrate on how developers use tools and the asset pipeline, 
and thus identify and react to workflow issues, such a bottlenecks in production or crunch, that slow productivity or increase the likelihood of errors. For example, developer-facing metrics such as Bioware's Skynet measure the efficiency and productivity of the development team, making this process visible to team members and managers (Zoeller 2010).

Customer-facing metrics are generally used to uncover design issues and analyze player behaviour once the game has been released, allowing developers to identify and react to issues such as bugs post launch, to understand the market and how their game was received, and thus to learn for future game development. Heat maps are commonly used to aggregate the behaviour of many players and play-throughs, and thus highlight certain flaws in design, such as bottlenecks where players experience unanticipated difficulties (Pruett 2010).

\section{Illustration 10.1: "Narrows" Halo 3 heat map}

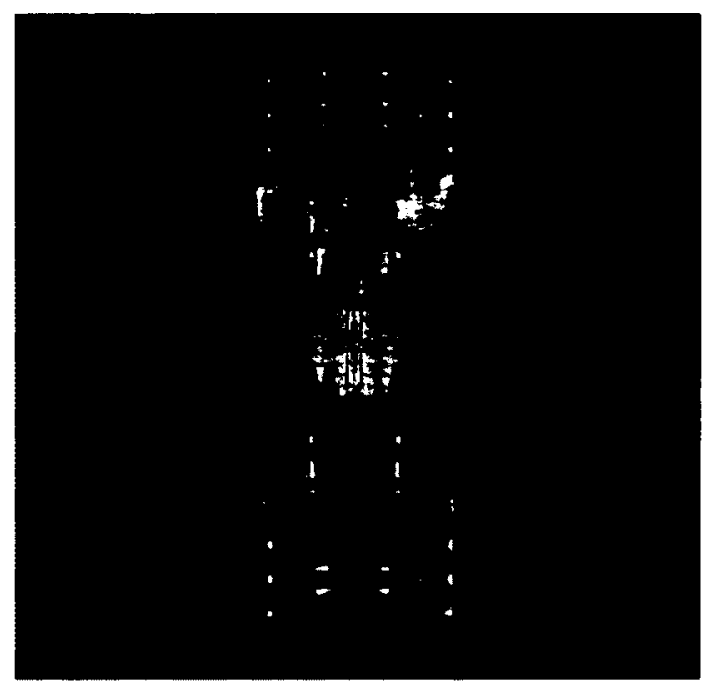

Source: http://halo.wikia.com/wiki/Narrows 
For example, this heat map is from a multiplayer level of Halo 3. Player deaths, represented by the lightened areas, skew towards the top half of the map. This indicates to designers that the team invading from bottom half has an advantage. Accordingly, the weapon placements and terrain can be tweaked by the designers to give both sides an even chance at winning. Besides heat maps, metrics are also used in Core sectors to classify player behaviour into different player types. ${ }^{161}$ Another instance of behaviourbased metrics can be seen in matchmaking systems for multiplayer games, such as XBox's Trueskill, that assess the player's skill level and matches them with other players, working under the assumption that players with similar skill levels will derive more satisfaction from the game (Medler 2009).

Moreover, specialized visualization tools are being developed to help designers make sense of the massive amounts of telemetry data being collected (Medler et al. 2011; Moura et al. 2011). Developers can cue up the data from any single player, determining the player's travel paths, time spent in each area, time spent interacting with NPCs, maps, and menus, as well as which items were collected. A final example of how metrics are used in core games is the experimental use of biometrics. Current uses of biometrics combine player-testing in a controlled lab setting with the collection of data such as heart rate and electrodermal activity to assess player engagement and response. ${ }^{162}$ This data

161These methods draws inspiration from Richard Bartle's four player types and their subsequent updates (Bartle 1996; Yee 2006a). In MMOs such as EverQuest, Dark Age of Camelot, Ultima Online, Star Wars Galaxies and Eve Online, empirical research on player types is achieved via player surveys (Yee 2006a; Nutt 2011b). In contrast,other researchers use automated data collection to recognize patterns in how the game is played, sidestepping "the traditional exhaustive user and player testing procedures used in the games industry" (Drachen, Canossa, and Yannakakis 2009:8).

162Most notably, Valve has experimented with biometrics, measuring the electrical resistance of players' skin to gauge interest in Left 4 Dead. The data was fed into Valve's AI 'director', which in turn upped the challenge during lulls of activity. Valve intends to use biometrics to facilitate multiplayer games, as they found that users were more engaged when they could see how they affected their opponents' biometrics levels. Similar experiments with galvanic skin response and breathing rates have be used to determine 
ostensibly provides objective data on the emotional states of players. Ultimately, the goal of biometrics research by companies such as Sony and Valve is detecting the emotions of players, designing games to leverage player emotions, and discovering optimal arousal rates to increase player retention (Crossley 2011i).

\subsubsection{Metrics in Casual Sectors}

Game development is no longer recognizable to the traditional modes of production detailed in Chapter Six. As put by EA Playfish's Tom Mapham ${ }^{163}$ "What they're doing looks a lot more like e-commerce than game design (as quoted by Alexander 2011). The new giants of the game industry, such as Zynga, have little in common with developers from Core, "Today scores of analysts continuously sift Zynga's vast customer data flow in real-time, segmenting their audience, optimizing customer acquisition and passing intelligence to designers and programmers to improve the customer experience, and, ultimately, drive up sales (Gibson 2011).

The desire for more data is never-ending, as evidenced by this interview with Lou Castle $^{164}$ when he was VP at Zynga:

Gamasutra: When designing a social game, what sort of data do you find most valuable in terms of metrics?

Castle: The data you didn't gather! Seriously, it is critical that you collect, as quickly as possible and allow for analysis of as many things as you can think of. Of course you need to balance scale, performance, storage etc. but in the end, your success will depend on your ability to gather information immediately and act quickly on what you find. (Castle 2011)

what makes games such as Dead Space 2 and Resident Evil 5 scary (Windels 2011).

163Mapham made this comment in regards to creating the Facebook version of the The Sims franchise. On a daily basis Playfish traced over a terabyte of player data, with a team of analysts and product managers running continuous usability tests and market research.

164 Castle is an industry icon. Co-founder of Westwood Studios, he is famous for creating the Command \& Conquer series. 
Castle's quote is telling in its emphasis - success in game development is not dependent upon intuitive design or the creative genius of developers, but rather how much "intelligence" can be gathered on players, and how quickly the company can act upon this intelligence.

Unlike Core development, the historical influences on the development of metrics in Casual games come from a very different direction. Metrics came to Casual games via the web developers who created the first early Facebook games. ${ }^{165}$ The evolution of metrics in Casual games was fuelled by the need to track the number and demographics of users, and not by playability research. ${ }^{166}$ This essential data determined advertising revenues, as in order to sell screen space to advertisers, publishers needed to quantify and guarantee the number of eyes that would see that ad. However, publishers soon realized that metrics could be refined to learn what attracted the greatest number of players, and thus cater to economies of scale with F2P games. Taking cues from the a/b testing in web design, Casual developers simultaneously rolled out different versions of a virtual object, mechanic or entire game to see which was more effective.

As technology has evolved, so too has player monitoring. ${ }^{167}$ Kontangent, whose customer list includes EA, Ubisoft and PopCap Games, provides a visual diagram to

1650 course, web designers were playing a very different game than that of traditional game designers. Success is measured in terms of page views, player numbers, and potential revenues, not in introducing critically acclaimed or innovative game play.

166Both the platform and design of Casual games facilitates gathering user data. Flash games in browsers are, by definition, always online, and thus easily send back continuous user data to the host.

167Early metrics providers simply tracked the number of users loading a particular game. Processing and bandwidth constraints meant they sampled a tiny proportion of users and then extrapolated their findings. However, the relative simplicity of flash games facilitated gathering more and more precise data. For example, analytic services for the Casual sectors include tracking player achievements, microtransactions, and player behaviour, including data on how often in-game advertising is clicked (Medler 2009). 
show the evolution of analytics services, below.

\section{Illustration 10.2: The shift from web analytics to social analytics}

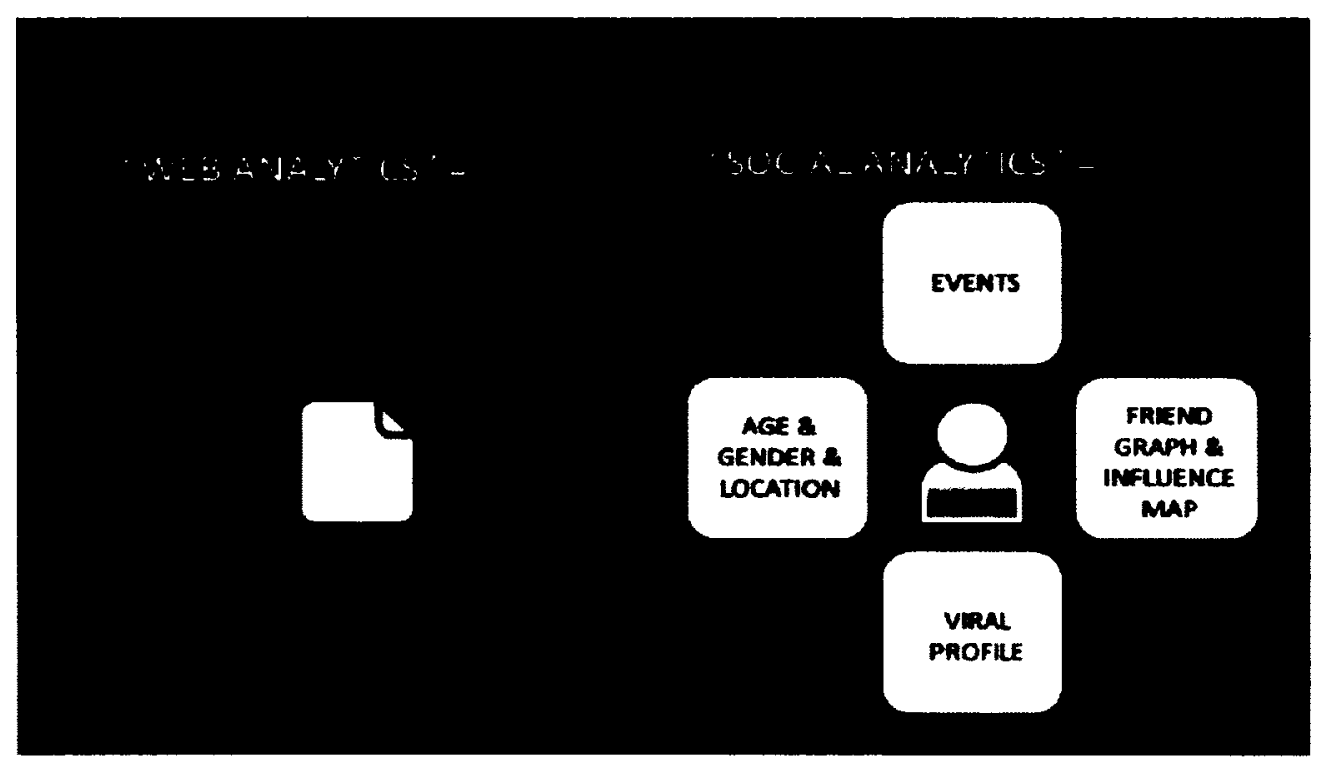

Source: (Lai 2011)

Whereas early analytics providers provided data on general trends, counting page views and session time, with Facebook and the preponderance of games linked to social networking services, companies have moved from tracking the masses to tracking individuals, in a shift towards "Social Analytics"(Lai 2011).

Facebook gaming was particularly fertile soil for the growth of metrics because for the first time in the game industry - quantitative metrics data could easily be linked to a wealth of real demographic data, providing the social context of players and clues to their behavioural motivations without having to resort to messy, time consuming, and expensive qualitative player-testing. ${ }^{168}$ As put by investment consultant Rick Gibson,

168Moreover, companies could contract the inexpensive services of a rapidly expanding number of analytics providers such as Mochibot, Nonoba, Kontangent, Playtomic, etc. Developers then use application programming interfaces (APIs) to hook into these companies' online analytics systems, automatically feeding recorded gameplay information to the analytics provider. The analytics provider then generates visual reports on a multitude of player behaviours and then sends them back to the 
"These new analytics tools allow unprecedented insight into your players - who they are, where they came from, what they play, what and how they buy, who they recruit on your behalf and, ultimately, how valuable they are to you" (2011). Instead of the ISP addresses and avatar user names, analytics companies have access to profile information that includes gender, age, location, religious preferences, friends lists, etc. In terms of Grimes and Feenberg's Theory of Ludification (2009), this allows for an unprecedented level of precision in terms of learning about players and their desires and bringing their non-game interests and social networks within the bounded magic-circle of the game.

New start-ups such as GameAnalytics ${ }^{169}$ promise considerable improvement over their competitors, especially in terms of shifting from simple a/b testing to more nuanced psychological profiling. Consequently, the company garnered this introduction in a Guardian news article: "It's a real-time service that continually monitors every player in any virtual world it's commissioned to work on. It's like CCTV constantly monitored by psychologists and statisticians" (Stuart 2011). GamesAnalytics promises "turnkey" solutions, off-the-shelf software solutions that are easily integrated by publishers and developers into each of their games. The emphasis of these systems is on monitoring individual players, creating psychological profiles based on their in-game actions, and then offering individualized marketing messages. This process is automated and thus works in near real-time.

Alan Miller, co-founder of Activision, the largest game publisher in the world and

publishers/developers in more decipherable formats (i.e. statistics, graphs and charts). $169 I^{\prime} m$ not sure if there is an "Emperor's Clothes" phenomenon at work with analytics start-ups. The evolution of GamesAnalytics, and competitors like GameAnalytics (yes, minus the "s") and Ninja Metrics deserves further study, especially since the latter two companies have roots in academic game studies rather than the marketing and game publishing industries. 
strategic adviser to GamesAnalytics, further illustrates the advances in metrics from $a / b$ testing with a somewhat disturbing dairy farm analogy:

To use an analogy - I have family in dairy farming - that approach that Kontangent provides gives a very high altitude view on the cattle, and it can tell you that 80 per cent of them are in Field A, and 20 per cent in Field B. It can also give you other global information, and that's very useful to publishers.

GamesAnalytics, because we do behavioural analytics at the individual player level, instead of looking at the entire herd, we look at each individual cow and can tell you a lot about that individual cow - and predict its behaviour.

Then, instead of just providing analytical results, we take it another step further and deliver immediately actionable marketing. In this analogy it would be sending a message to the farmer to have one of his field hands go out and milk a certain cow in a certain place - and tell you how much milk to expect from it. (emphasis added, as quoted by Elliott 2011a)

While colourful, this description of the heart of metrics - "milking players" - is representative of the general discourse arising from social games analytics. As put by journalist Keith Stuart, "Whereas traditional games are about creating big macroenvironments for player exploration, freemium is about micro-managing every step the player takes toward actually buying something" (Stuart 2011).

With the growth of cloud based metrics solutions that allow for flexible data storage, detailed real-time analysis of data flows is being applied to more and more game sectors. In 2011, a number of analytics service providers, such as Ninja Metrics and PlayMetrix, have started to offer more in-depth analytics to Core console and PC publishers. Veteran designer and modder Chris Birke ${ }^{170}$ speaks to the increasing sophistication of cloud-based metrics, especially those that track persistent identities and

170As a side note, Birke's modding roots run deep: he created the first "Quake Movies", popularizing Machinima and was also involved with the production release of Counter Strike (the Half-Life modturned AAA release). 
social graphs to customize games on a per-user basis:

The relationships between all the players, their in-game interactions, and the histories of players over time and across games can now be tied to a unique profile for each user. This massive well of information is where web analytics and game design begin to synthesize into something new ... Rather than just gathering information on player attendance, every aspect of player behaviour can now be collected as analytical data. Questions of what, when, who, and why can be asked, and a whole slew of predictive information can now be developed. (Birke 2011)

Successful game design thus becomes a game in terms of predicting what players will do next. This sort of challenge becomes very alluring to power gamer developers.

Metrics turn development into a god-game. ${ }^{171}$ The developer, using the data from metrics, controls the game on a large scale, using their omniscient knowledge and ability to tune the game on the fly. Raised on strategy, simulation, and god-games, developers use metrics to turn their own work into a game of indirectly managing players by changing the environment of the game. Accordingly, metrics - especially the fine-tuned metrics used in Casual games - allow developers to play with governance in unprecedented ways.

Not surprisingly, games are idealized tools of governance. Due to computational logic - the belief that once you introduce a computer into the mix, the rules become rigid - this governance, while limited, is more tractable in game worlds than in offline spaces (see Whitson 2010a). As put by designer Dan Cook in comparing the messiness of realworld governance to the governance in games, the use of metrics in games presents considerable appeal to developers because, as long as long as the methods of

171God-games, such as Black \& White and Spore, are a sub-genre of AI games. They are similar to construction and management simulation games such as The Sims but, instead of directly telling characters what to do, the player takes the role of an omniscient god that can only indirectly influence characters' behaviour by changing the environment (e.g controlling the weather, transforming the landscape, etc.) 
measurement are reliable, developers can "create reproducible and scalable patterns of human behaviour to a degree that has hitherto been the downfall of empires". He provides further clarification on this appeal of computational logic:

Once I've tested a game on 10,000 players, I know with reasonable certainty what effect it will have on 10 million. The playtesting and metrics that drive modern game design are as much about finding the predictable bounds of human behaviour within a system as they are about finding a system that is enjoyable. (Carrots and honey when properly applied are quite successful at constraining a populations' behaviour while still honouring free will.) (Cook 2010)

What makes games special from other attempts at computer-moderated governance is that games "explicitly deal in the intricate aspects of autonomy, motivation, goals and other psychological circuitry that seem so central to our illusions of identity and will" (Cook 2010). For players, they are spaces of seduction and governance because they are spaces of fun, creativity, and exploration.

On the developer side, the instrumental play involved in metrics-driven design comes with the challenge of using data and subtly manipulating the game environment to learn how players 'tick'. Developers have an increasing understanding of how, on a psychological level, game design might be used to promote both positive and negative behaviours (see E. Robinson 2011; Blair 2011; Birke 2011; B. Phillips 2009). While alluring, the power to govern player's behaviours comes with an increased ethical burden, as unlike god-games, developers are playing with real people, not NPCs.

To link this back to the discussion of Foucauldian governance ${ }^{172}$ in Chapter Three, the collection of data in games, maps and thus creates a territory, rendering visible the

172In particular, I am drawing from Nikolas Rose, who in turn is drawing from Bruno Latour and ActorNetwork Theory. 
space over which government can be exercised. Metrics become a inscription device. They are modes of objectifying, making, inscribing and preserving otherwise ephemeral and subjective visions (i.e. what exactly are players doing when they are playing?). Inscriptions such as maps, charts, diagrams, graphs and now metrics, make things stable, mobile, durable, comparable. Inscription devices are thus powerful tools. As phrased by Rose, they are rhetorical:

That is to say that a map, a chart, a table, a diagram is a little machine for producing conviction in others. Inscriptions thus produce objectivity in a way that is different in its nature and its uses from speech and the bearing of witness. Inscription devices are 'intellectual techniques': material techniques of thought that make possible the extension of authority over that which they seem to depict. (1999:37)

In a few simple and elegant statistics, metrics define what matters, distilling the complexities of the world and human behaviour into something more tractable, such as measuring 'fun' as a function of ARPU calculations, or determining the human worth according to the money they spend in-game and whether they are likely to spend more. The escalating surveillance in games and gamification movements is important not because it identifies a real name, address, or location. For the most part, player's privacy and right to anonymity is protected. Surveillance in games matters because metrics are inscription devices that render people and their actions governable, regardless of whether their real name is known or not.

\subsection{Who Counts in Game Design? Why surveillance and god games matter}

Metrics turn games into spaces of social sorting and commodification. They allow developers to expand into niches beyond the mainstream, or divide the mainstream into 
more manageable developmental targets. Of course, not all demographics are of equal worth. On average, $98 \%$ of Casual game players do not spend a single penny. This means that for every 1000 players, there are 980 "freeloaders". Of the $2 \%$ of paying players, most only spend a dollar or two, while a small fraction of them spend much more. Knowing more about what player types spend money in-game, and what items they are willing to purchase, and what entices a non-paying player to become a paying player, are essential tools that help shape the design of the game. Consequently, players are tracked and split into categories depending on how much they have spent in-game. These highspending players are now classified throughout the industry as "whales". ${ }^{173}$

Developers are consequently advised that "If you're a game designer, your main take away is that very few transactions - and consumers who complete those transactions - make up the bulk of your revenue. Therefore, your "meta-game" should be about whale hunting" (Valadares 2011). Once developers identify what game mechanics or virtual objects encourage user spending, especially for whales, the direction for future design is set: producing improved variations of profitable items.

The increasing sophistication of metrics means that analytics providers and developers cannot only pinpoint and cater to big spenders, but can also locate players who exhibit behavioural patterns similar to whales and thus might become big spenders given the right encouragement. ${ }^{174}$ For example, GamesAnalytics markets their service as

$173 W$ hales are those players that spend over $\$ 1,000$ on a game, with some spending over $\$ 20,000$. Nicholas Lovell collected industry reports of ARPPUs (average revenues per paying users) and provided the following categorizations:

1. Whales: for every 1000 players, 2 are whales, spending an average of $\$ 20$ per month;

2. Dolphins: for every 1000 players, 9 are dolphins, spending an average of $\$ 5$ per month; and,

3. Minnows: for every 1000 players, 10 are minnows, spending an average of $\$ 1$ per month.(Lovell 2011b).

174Conversely, Lovell speculates that developers such as Zynga also shift the "freeloaders" to a series of 
a way to identify freeloaders who exhibit similar psychological patterns to whales. They then target these players, hoping to turn them from players who spend nothing to whales who spend thousands of dollars:

[O]ur software will scan every active player - that can be thousands, hundreds of thousands or even millions of concurrent active players... and that's a big technical challenge. We evaluate all of them to see if they're getting close to any of the desired behaviours, and when they start to get close, we signal the game to deliver one of the pre-defined marketing messages to that specific player. They're individually assessed and targeted marketing messages designed to accomplish specific things. (Elliott 2011a)

Essentially, what GamesAnalytics is doing is whispering into the ears of players, nudging them to consume. The vast majority of these players have no inkling that these whispers are individually crafted just for them based on sophisticated psychological and behavioural profiling.

While whales and potential whales are the most recognizable demographic for targeting, monetization is not the only driver of social analytics. As Birke summarizes in his assessment of behavioural profiling in game design, not all players are of equal worth. Just like grade school, it pays to be friends with the popular kids, and thus "influencers" are the new goldmine for targeting:

It's very valuable to identify your early adopter players and the opiniongenerating players who act as social hubs. These players are the agents of virality, and your means to success. Identify them and let them know how special they are with rewards and privilege. Tell them early about your next game, and encourage them to move on to it. The rest will follow. (Birke 2011)

The focus on identifying influencers comes from surprising avenue - academia, particularly the burgeoning field of social networking analysis (SNA). Ninja Metrics, one

different games on a two month rotation, hoping that one of these games will convert the freeloader into a whale (Lovell 2011a). 
of the newest analytics start-ups is founded by University of Minnesota computer science professor Jaideep Srivastava and Annenberg School of Communication professor Dmitri Williams. ${ }^{175}$ Instead of aiming their services at academics interested in online socialization or developers looking to understand play, Ninja Metrics is decidedly marketing-focused. ${ }^{176}$ The system is predominately marketed as "A state-of-the-art patented system that tells you who matters most in your network" and is also capable of tracking player behaviours such as spending, quitting, cheating and being hacked, as well as creating heat maps (Ninja Metrics 2011).

Ninja Metrics' Katana Engine is the ultimate social analytics tool, tracking both spending patterns and social interactions to pinpoint influencers:

Social networks work more virally than anyone thought. Behaviours spread over time, for everything from buying, quitting, and even moods. Knowing how things spread tells you who matters in the network. Some people are more influential than others. We quantify everyone, enabling you to see the whole network, and then deep down, who is driving the behaviours We actually tell you the value-for each person - based on their influence. Welcome to Ninja Metrics...(emphasis added Ninja Metrics 2011).

Quite literally, the analytics engine and visualization tools turn players into dollar signs, as evidenced by Katana's demo screenshot, below. Those nodes that are at the centre of social hubs are identified as influencers. As the example above shows, influencers can be whales, but this is not always true. The red nodes ${ }^{177}$ are players who "are costing you money by driving others out of the game" (Ninja Metrics 2011).

175Ironically, this is the same Dmitri Williams that critiqued the financially motivated, hits-driven structure of the console industry in Chapter Six.

176This may be a necessary marketing move to attract initial investment.

$177 \mathrm{ff}$ this illustration prints in black and white, the few red nodes encompass a range of spending values, from $\$ 20$, to $\$ 97$, to $\$ 298$ and $\$ 233$. Accordingly, simple assessments of how much each player spends do not equate to their 'true' worth, as red undesirable players spend much more than the average player. 
Illustration 10.3: Ninja Metrics and a player's true worth

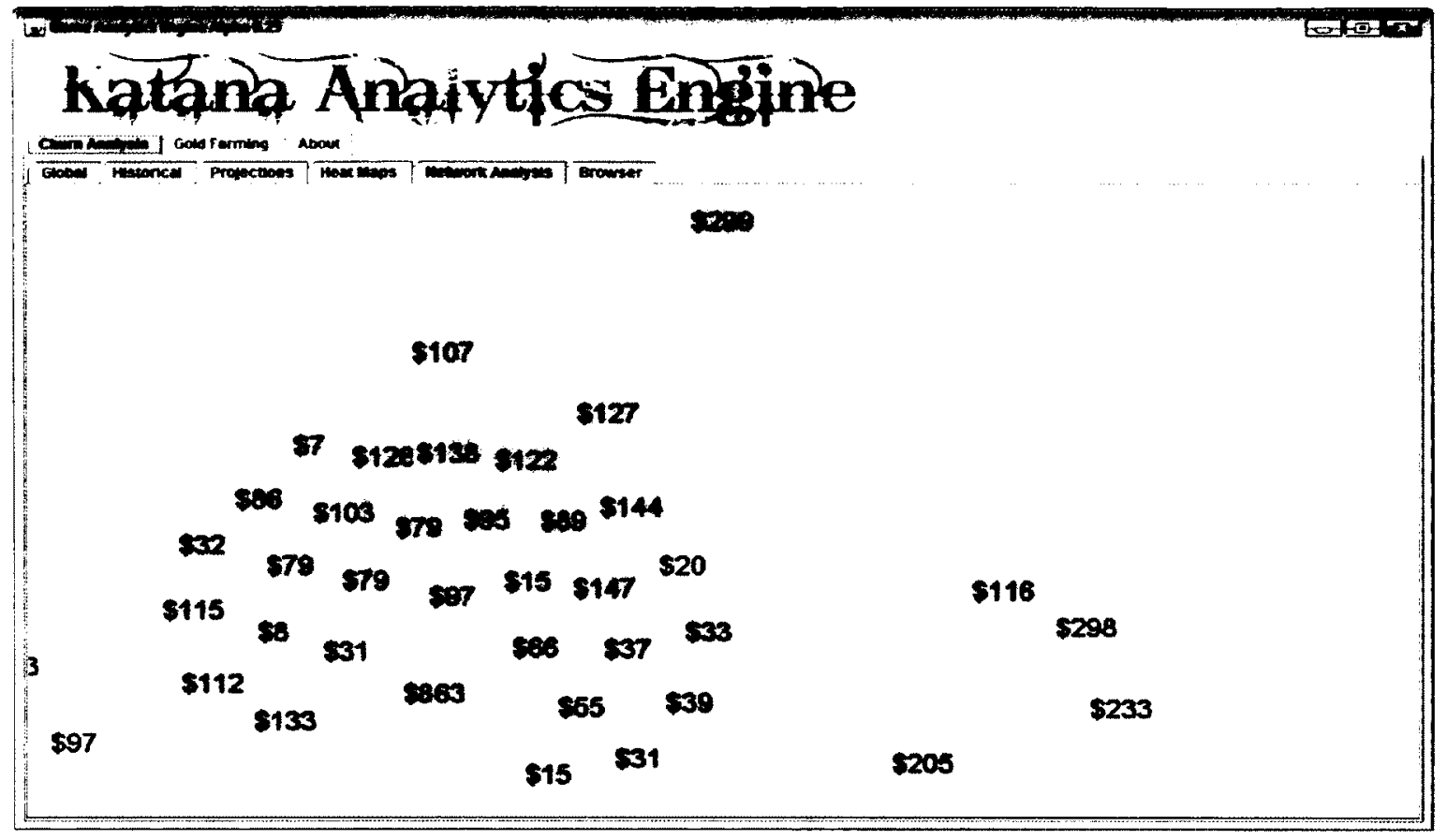

Source: (Ninja Metrics 2011).

Ninja Metrics promises publishers improved consumer relations. Customer service representatives (CSR) are immediately able to call up SNA profiles and player histories for each user. By immediately knowing which player's opinions matter more, CSRs can perform customer service triage: "With each players' Network Value and history charted, your CSR team can make important on the spot decisions of specific interventions to take with individual customers, depending on their network value, such as providing them a discount or free item for troubles they have experienced" (Ninja Metrics 2011). In effect, SNA engines like Katana are a digital redlining tool. Whereas insurers and bankers once refused service to people because they lived in areas deemed to be a poor financial risk, game 
publishers are now - unbeknownst to players - able to streamline and cater, reduce and cut services based on predicted spending patterns and behavioural categorizations.

This is not an isolated case. Gabe Newell, co-founder of Valve ${ }^{178}$, half jokingly speculated on what metrics means for games:

[T]his is something as an industry we should be doing better - is charging customers based on how much fun they are to play with. Some people, when they join a server, a ton of people will run with them. Other people, when they join a server, will cause others to leave. We should have a way of capturing that. We should have a way of rewarding the people who are good for our community.

So, in practice, a really likable person in our community should get Dota 2 for free, because of past behaviour in Team Fortress 2 . Now, a real jerk that annoys everyone, they can still play, but a game is full price and they have to pay an extra hundred dollars if they want voice...Each one of these people should represent a different monetization scheme for the community as a whole.....That's the best thing in the long run for the community. (Crossley 2011j)

This sort of digital redlining in games is what makes the New Spirit of Capitalism so insidious. With the networked models of the Projective Cité, everything is connected. There is an erasure of the separation between work and non-work activity that deprives individuals of their 'private existence' (2007:465). There is no space outside of work and the commodity sphere. Even while playing a game, our personalities and our play styles whether we are "likable" or a "jerk" - can be measured and leveraged in the pursuit of profit.

While the Second Spirit of Capitalism and the Taylorism of work is responsible for treating human beings like machines, the New Spirit of Capitalism places "the most

178Valve is also driving the biometrics research noted earlier in the chapter. I can only speculate what happens when one's biological flows - our heart rate and arousal levels - start to enter the domain of large-scale metrics. I can certainly envision a future where how much you pay to access games and how long you are able to play them depends on your fitness level. 
specific qualities of human beings - their emotions, their moral sense, their honour, and so on - directly in the service of the pursuit of profit" (Boltanski and Chiapello 2007:465). Everything - from our social relationships to the time we spend playing games - is commodifable. We never leave capitalism behind us. Every moment of our lives, every action we take, can be leveraged in some way to make us better workers and consumers. There is nothing beyond the sphere of commodification. Like smoke filling our lungs, we can breathe nothing else. We have no critical distance, no breathing room, from which to criticize the forms capitalism has taken.

What makes predictive governance in games so disturbing is the opacity of this commodification. Player recognition of surveillance and resistance to it is focused on comparatively unsophisticated uses of telemetry, such as logging player deaths to create heat maps and using aggregated data of play-throughs to determine whether to cut certain features, such as the ability to play as a female character in Mass Effect (Bruno 2010). In fact, in over four years of gathering data on the game industry, I only found a few instances of player resistance to profiling and surveillance. ${ }^{179}$ Osborne Clarke lawyer Jas Purewal supports this finding, arguing that many players do not understand the way their data is used to influence future behaviour: "It means you've got data, you've got metrics, you know things about your customers that you can work with, but again there's a

179For example, in response to Just Cause 2's heat maps, players half-jokingly speculated about their game logs being used against them in non-game contexts:

Consumatopia: I forsee prosecutors requesting a subpoena from video game developers so they can show the jury all the stuff the accused likes to do in video games. Certainly if I were selling health or life insurance I'd pay to get this data.

MiniMatt: Oh crap, you're right. My big brother sense was already all a tingle but I can see prosecutors (and the Daily Mail) having a field day with stats gathered. Anyone else here nuke Megaton in Fallout $3 ? . .$.

Hallgrim: Oh my, what a wonderful way to provide evidence of someones "insanity". "Your honor, I have here in my hands, evidence of the defendant's dangerous psychosis!"*holds up Grand Theft Auto logs* (Meer 2011a) 
disconnect because players don't really understand that everything they do is being monitored" (as quoted by Handrahan 2011a). As of yet, most players are unaware that individualized, persistent player monitoring is indeed possible. Nor are they aware that individualized versions of games are released to players. There is no recognition that players may be categorized into groups based on their desirability as consumers. There is no recognition that players' future behaviours and worth are now being predicted, and there is absolutely no recognition that players are now being sent individualized whispers of marketing information and treated differently accordingly to how much they are predicted to spend, or how fun they are to play with. After all, this is only a game, right?

The opacity of data-based surveillance, coupled with its obvious utility in game design, is part of what makes metrics so hard to critique. The second half of this chapter focuses on how this reliance on data relates to the New Spirit of Capitalism and what this means for game design, particularly for the humans that make and play games.

\section{PART II}

\subsection{Innovations to the New Spirit of Capitalism}

To return to the New Spirit of Capitalism, Boltanski and Chiapello are interested in tracing how a new and virulent form of capitalism that relies on networked forms of thinking has an even more disastrous impact on the fabric of a common life than its predecessors, yet has managed to install itself smoothly and inconspicuously without any organized resistance from forces of opposition. Boltanski and Chiapello have been criticized for excluding the impact of technological changes from their analysis, 
especially the impacts of new information technologies. ${ }^{180}$ This second half of the chapter stands as a corrective to this. I focus on one technology - metrics - and how it influences the shape of the New Spirit of Capitalism, tracing how it changes the Projective Cité in ways that were not accounted for, nor predicted by, Boltanski and Chiapello.

To reiterate, Cités are different intuitive notions of justice that people bring to their encounters with the world of social relations and objects. They are "orders of worth" that contain systematic and coherent principles of evaluation (i.e. justificatory regimes). Each Cité uses a distinctive "grammar" of justice. In the Projective Cité, this grammar is founded on the "network", a harmonious figure of natural order that conveys an understanding of how the world really works. Cités (Civic, Market, Inspirational, Renown, Industrial, Domestic, and Projective) co-exist in the same social space, with some more dominant than others. In particular, the game industry operates according to logics rooted in the Projective Cité and the Industrial Cité, with the Projective Cité quickly gaining precedence. ${ }^{181}$

The Second Spirit of Capitalism promised an escape from the Domestic Cité, promising job security, meritocracy, and an end to nepotism. In turn, the New Spirit of Capitalism promises an escape from large bureaucracies and the cold rationalizations by which they operate, replacing alienated labour with networked models of production that reward autonomy, self-governance and innovation. Yet, the New Spirit of Capitalism has clear drawbacks. Material and psychological security is traded for mobile autonomy, the

180They argue that this was an intentional move. By not isolating technology as an independent variable in the development of capitalism, they hoped to break with a "fatalistic vision of technological determinism" (2007:xix).

181This is not to say that other Cités are absent. For example, it could be argued that the values of the Domestic Cité play a role in Japanese game development (see Ernkvist and Ström 2006). 
barrier between work and leisure time is collapsed, and networked projects suffer from the difficulty associated with assessing reputation and rewarding contributions fairly.

The metaphor of the network has a powerful structuring aspect on the Projective Cité. With the network metaphor comes new values and measures of success, new understandings of what is 'fair', and new visions of how businesses should operate. Instead of the game industry - or any industry, for that matter - operating as an efficient industrial machine, the workforce is seen as a living, complexly linked, constantly shifting network. Consumers are seen not as a mass undifferentiated market, but a dispersed web of individualized yet connected groups that needs to be catered to in subtly different ways. Whereas authority in the Industrial Cité is rooted in adhering to a managerial hierarchy and accepting orders from above, the Projective Cité emphasizes that 'the customer is king', not the management. As Boltanski and Chiapello argue, this customer-centric view presents clear advantages:

[O]n the one hand, it inflects self-control in directions conducive to profit, given that a firm's differential ability to satisfy its customers is an essential ingredient of success in a competitive economy; and on the other, it transfers some of the control exercised by superiors in the 1960 s to customers. (2007:81)

In the game industry what this means is that numbers - both in terms of players and profits - become paramount. These numbers articulate the demands of the populace, and despite what individual developers may want, the customer is king. If millions of players are playing social games, then they cannot be wrong. Not surprisingly, this has distinct implications for creativity.

Creativity and innovation take distinctive network forms. In the industry, new 
connections between individual members and sectors are continually being made, leading to emergent forms of innovation. Both the Projective Cité and the Inspirational Cité are premised on creativity and innovation, emphasizing the uniqueness of being and things, but the Inspirational Cité focuses on individual creators and artists, while under the Projective Cité, people are only creative when they are linked to others. For example, as we saw in Chapter Nine, creativity and innovation in social games comes from harnessing networks of players, not individual designers or managers.

This leads us to a key evolution in the New Spirit of Capitalism that was not accounted for by Boltanski and Chiapello: replacing the "great men" and "visionaries" that drive the Projective Cité with crowdsourced data sets. In Boltanski and Chiapello's account of the New Spirit of Capitalism, small autonomous teams working on projects have a shared meaning - they know what they must do without being told. This shared meaning is cultivated by their leader, who has a vision and knows how to communicate it and get others to support it. As Boltanski and Chiapello acknowledge, "This is doubtless the weakest link in the new mechanisms, for everything rests on the shoulders of an exceptional being; and it is not always clear how to train or even recruit such beings, especially in sufficient numbers, since every firm needs them" (2007:76). With information technology, specifically metrics, industry - not just the game industry, but any industry that utilizes similar data analytics - can do away with a reliance on leaders. Following the discourse of trusting the consumer, all industry has to do is to find out what each consumer wants and then cater to each of their individual desires. ${ }^{182}$ As we saw in

182In 1999, at the time Boltanski and Chiapello published the first French edition of The New Spirit of Capitalism, datamining technologies to make this possible were still nascent. Accordingly, it is perhaps not surprising that they rely on so heavily on fallible human visionaries rather than automated crowdsourced ones. 
the previous chapter, the social game industry has excelled at this.

This leads us to another evolution in the New Spirit of Capitalism: the revival of rationalism. As the growing field of Social Network Analysis (SNA) argues, the emergent patterns, currents, and forces of power that underlie networks are largely invisible to the common eye. They are only made visible when they are locked in place and parsed via inscription technologies such as metrics. SNA practitioners and game developers both apply a 'hard science' approach, using software to quantify and measure what was previously unquantifiable (e.g. the ties between people) (see Knox, Savage, and Harvey 2006). This is at play with the gamification practices detailed in Chapter Three: our own bodies, actions, and relationships are too complex. We trust in machines to parse these flows of data, and to give us intelligible feedback on how to master our selves. On a macro level, this is at play with human networks of consumers. Metrics and other data collection practices replace human - and thus fallible - vision with quantifiable - and thus verifiable - numbers directly from consumers.

This is what the wisdom of crowds is all about. ${ }^{183}$ It replaces the rationalism that was objected to in the Second Spirit as "cold, calculating administrative rationality" (Boltanski and Chiapello 2007:78) with something that is much less open to criticism. Instead of relying on rationalizations handed down from managers at the top of lumbering workplace hierarchies, this new form of rationalization comes from the bottom-up: it comes directly from the consumers, making conclusions and management directions drawn from this data much harder to criticize. In addition, the numbers

183James Surowiecki's The Wisdom of Crowds (2004) argues that a diverse collection of individuals make better predictions and decisions than individuals or experts. In contrast (and more in alignment with my own beliefs) Jaron Lanier argues that the wisdom of crowds is best suited for problems that involve optimization, but is ill-suited for problems that require creativity or innovation (2010). 
churned out by metrics are made visible, thus avoiding the Kafkaesque opacity that comes with bureaucracies.

Not only does this new rationalism solve the problem of unreliable visionaries, it also presents a solution to the job insecurity that has so far characterized the New Spirit of Capitalism. Job insecurity is partly reliant on the fickleness of consumer tastes created as the demand for authenticity on the part of consumers leads to rapid cycles of infatuation and disappointment as they search for unique, authentic products (Boltanski and Chiapello 2007:445). Firms such as game publishers attempt to predict what the market - and these fickle consumers - desire, but these predictions often fall flat. In the game industry where only $4 \%$ of games that go into production and $20 \%$ of launched games actually make a profit, the survival of entire companies depends on the ability to predict whether the one game they are working on will be a commercial success (Irwin 2008). Accordingly, a better ability to predict consumer desire via increasingly sophisticated metrics is linked (at least in game developer discourses) to the continued survival of firms, thus leading to individual job security.

Metrics solve another problem associated with the Projective Cité. Small, agile, networked pods of workers can result in too much worker autonomy (i.e. the project can quickly get off track, or lone individuals can, for selfish reasons of their own, torpedo the success of the project). Boltanski and Chiapello emphasize the inculcation of new business ethics and self-control to avoid these problems (2007:95). But technology offers a new solution. Metrics extend novel forms of control over individual developers: there is less direct monitoring and face-to-face control, but other, less overt and thus less 
objectionable, modes of control are established in their place. As exemplified in Chapter Three, metrics becomes an essential tool for internalizing self-control. This applies just as much to developers as it does to players, allowing them to reduce direct hierarchical supervision by substituting self-control. While granted a greater degree of autonomy than workers on the Taylorist factory floor, developers are increasingly "monitored by computer systems that not only define the relevant categories recognized by the system, but give them a 'prescriptive' force, which leads to structuring tasks through 'grammars of action'"(Boltanski and Chiapello 2007:431). Developers have freedom in how they go about their work, but are generally compelled to use the quickest way of working (and not the one that suits them best). Moreover, developers are tightly controlled by the market. They must meet consumer demands and tight deadlines, thus space for iteration, experimentation, and free play are limited. The more data on what consumers 'want' means increasingly less space for creativity. In the next section I highlight what this means for game design and spaces of play.

\subsection{What This Means for Game Design}

In the Projective Cité, in particular, metrics bolster a particular regime of truth with authorized knowers and particular forms of knowledge that are predicated upon quantitative data. In the game industry, data is what is authorized to 'speak' and not individual developers or players. Metrics are what counts as truth, allowing a positivist scientific discourse to now overlay the production of games. Metrics leads to a quantification in culture industries such as game development, encouraging specific 
forms of creativity as well as specific forms of governance that look to predict consumers' behaviour using 'objective' data. Quantification and the automation of surveillance and data analysis have now become key aspects of the New Spirit of Capitalism. This ultimately changes what game design looks like and how both players and developers are treated.

\subsubsection{From Individual to Dividual}

In this section, I will argue that metrics lead to the creation of tractable subjects and the valuation of quantitative objective data over other forms of player feedback. In the shift from a static retail product to selling a long-term service, opening communication channels with players is depicted as one of the most important things a developer can do in order to guarantee long term success (Holtman 2009). However, with the increasing reliance on player metrics, there comes an unintentional devaluation of more qualitative forms of feedback, and a consequent devaluation of players as unique agents. This devaluation occurs in four general steps:

1. Metrics prove that players do not behave as developers and designers predict;

2. Metrics prove that players do not behave they way they say they do in qualitative feedback, such as forums and playtesting;

3. Accordingly, players do not know what they want; and,

4. Thus, compared to metrics, players are untrustworthy.

The rise of social and mobile games has been directly attributed to the connectivity and the "close relationship with players" that are enabled by metrics (Gaudiosi 2011).

According to Ed Fries, metrics are likely the biggest change that's affected the gaming industry over the last few years: 
It's not just that we have digital distribution to our customers. It's that we have this incredible two-way connection that we've never had before with our customers. We've gone from a situation where we dream up a game, we spend three years making it, we put it in a box, we put it out in stores, we hope it sells, to a situation that's incredibly more fluid and dynamic, where we're constantly modifying the game with the participation of the customers themselves. (as quoted by Bishop 2011)

Game industry executives, publishers and developers laud the improved communication with players that metrics facilitate; however, upon closer inspection, this communication seems more a tool for governance, prioritizing the monitoring of dividuals and framing this surveillance as communication. In this case, the "participation" that Fries is referring to is inadvertent on the part of the players - automatically collected metrics act as tractable stand-ins for more active forms of participation.

Metrics, and not qualitative feedback are depicted as the most accurate way to learn about players. For example Andy Tian, the general manager of Zynga Beijing, espouses the following view of metrics:

[Y]ou understand more about player behaviour through metrics. One way the metrics can definitely help you is to understand how players really behave, how they really think. Another way is you always ask your user to get qualitative feedback.

Quantitative measurement and qualitative feedback need to go hand in hand. Metrics can tell you how they're doing it, but not why are they doing it. Sometimes you may not be able to answer that - like qualitative feedback from users sometimes doesn't really represent what they actually do. (emphasis added, as quoted by Nutt 2011a)

While Tian gives lip service to qualitative feedback, ultimately metrics are deemed as preferable sources to learn about how players think and feel. Importantly, while Casual games companies such as Zynga and Crowdstar employ community managers and keep close track of qualitative fan comments and forum posts, they note that quantitative 
feedback is a more accurate predictor of current and future player behaviour. It is only when metrics fail to provide the needed context (e.g. why are people getting stuck on this level?) that developers then turn to forums and qualitative data for clues (Nutt 2010, 2011a).

While many developers, such as Eve Online's CCP Games, still emphasize the importance of qualitative feedback and face-to-face interactions with players (Nutt 2011b), there is increasing acceptance that as player populations grow, it becomes more difficult to maintain relationships and communication channels with players without the use of metrics. ${ }^{184}$ In an economy of scale that undergirds MMOs and Casual games alike, communication (collecting and responding to feedback) is automated.

Discourses on the use of metrics in games are commonly coupled with 'discoveries' about the failures of traditional modes of player communication. Particularly, there is a common mismatch between what players say they want and what they do in the game. For example, aggregated playthrough data evidences that the majority of players never finish games, despite the fact that many gamers are clamouring for larger, harder games. As put by John Davison, Vice President of both Metacritic and Gamespot:

The problem is, the vast majority of gamers don't really behave the way they say they do. How do we know this? Because an increasing number of games incorporate telemetry systems that track our every action. They measure the time we play, they watch where we get stuck, and they broadcast our behaviour back to the people that make the games so they can tune the experience accordingly. (Davison 2010)

184For example, J Todd Coleman, creative director and VP of tween MMO Wizard101, discusses the influence of social games on player feedback in MMOs. Ultimately, the company's direct player-testing is decreasing as the game's population increases, "We still try and get feedback from the players, though it is becoming harder when you got 10 million. Separating the signal from the noise becomes a real challenge that we'll continue working on that and try to improve on that" (as quoted by Caoili 2010c). 
For Davison, in a cash-strapped Core development scenario, the best solution is not giving players what they ask for, but rather to focus on producing shorter games. ${ }^{185}$

Industry discourses are rife with examples of gamers not doing what they say. As a result, the use of metrics parallels growing distrust of player forums, the most popular venues for game-specific qualitative feedback. For example, at GDC 2011, Ben Cousins, general manager of EA's Easy studio discussed the surprises that came with launching Battlefield Heroes (Nutt 2011c). When faced with lower than predicted revenues, the company experimented with a range of new monetization methods, including the introduction of weapons that could be purchased through microtransactions. While they were met with overwhelmingly negative responses on player forums, the company proceeded with the unpopular changes in a last-ditch effort to generate revenue. ${ }^{186}$ Overnight, there was a 100 to 200 percent jump in daily revenue with the new guns, and three times as many players as before "converted" from freeloaders to spenders.

To Easy Studios, this meant "there was a mismatch between what was being said on the forums and what was happening in the data" (Cousins, as quoted by Nutt 2011c). This finding aligns with similar accounts from Brian Reynolds at Zynga, who discusses the general distrust of forum data, especially negative feedback on forums: "First of all, we would have to say, 'Well, is that good or bad? Is it just anecdotal, that it's just the

185For example, Davison states that less than $5 \%$ of a game's audience plays a title through to completion, whereas more than $90 \%$ of players play for just four or five hours. I disagree with Davison. Players are willing to spend $\$ 70$ on a game because of the perceived value of said game. While they may play 5 hours only, the fact that a large environment awaits them with missions yet to complete and detailed worlds to explore is still a selling point. Short 4-5 hour games have a much tougher time attracting paying customers unless their price is drastically reduced, especially as they are now competing with F2P games.

186The studio was facing job cuts by parent company EA, adding to the pressure. 
loudest voices or the craziest people saying those things or is it actually true?' ... Well now with social games we know that stuff. It's very easy to figure that stuff out. We can run a test" (as quoted by Brightman 2011b). Accordingly, while Zynga relies on forum data to provide context for metrics, metrics provide testable and thus more objective data, especially in comparison with the "whiners" and "crazies" that generally populate forums. Players cannot be trusted, while data does not lie.

Accordingly, Easy decided to investigate whether any forum feedback could be trusted. Their analysis of both game metrics and forum metrics showed that $78 \%$ of players never visited the forums, $20 \%$ "lurked" but never posted, while only $2 \%$ of the players actually posted on the forums. It would have been easy to ignore the forums altogether, labelling all posters as whining freeloaders. However, Easy Studios collected further metrics detailing which players posted on forums, what they did in-game, and how they spent their money. They discovered that forum posters spent on average $\$ 22$ more than 10 times the average user. Instead of concluding that forum posters were a profitable niche demographic that should be catered to, Easy Studios came to a very different conclusion: that these forum posters did not know what they wanted: "there seemed to be a disconnect between what they were saying - 'I will leave and never spend a penny' - and what they were doing - sticking around and spending a lot of money". In other words, companies like Easy Studios and Zynga believe that qualitative accounts are ultimately unreliable, and may run counter to actual spending habits. Accordingly, Easy Studios now prefers a "data-driven examination" of potential problems rather than relying on direct player feedback (Nutt 2011c). Rather than being an isolated case, distrust of 
qualitative user feedback is a common trend in the game industry (see Cook 2011b;

Caoili 2010b; Dallman 2011). ${ }^{187}$ Ultimately, following qualitative feedback and listening to user forums is depicted as bad for business, while metrics are depicted as revealing unseen truths. As the above example attests, developers readily agree that hard facts are to be trusted over subjective user responses.

\subsubsection{Designing Out Designers}

Ironically, developer and designer support for metrics comes with consequences for their own job security, as metrics are more successful than designers at both predicting and shaping player behaviour. As put by games journalist Christian Nutt, "That's what I find really fascinating. It's a well-known fact that what game designers might think is cool is not necessarily what users might like. But it's also true that potentially what users might think they want isn't exactly what they want" (Nutt 2011a). Just as evolving a game based on forum feedback is increasingly seen as a poor business decision, designing a game based on the creative intuition of developers and designers is seen as an untrustworthy, risky, and inefficient business strategy.

As put by Justin Johnson, developers "create a certain game experience that they want a player to go through, and I wonder how many boxed retail games are played

187For example, Playdom designer Joshua Dallman's experiences with Wild Ones echo that of Battlefield Heroes: "The qualitative feedback coming in was that the game changed too much for the player base and was now terrible - but the quantitative feedback for number of players, session lengths, and monetization told a completely different story, showing that players loved the new game, and it was thus an unequivocal success. In live game design you have to be careful not to design around the vocal minority, thereby ousting the silent majority. Those who played and stayed and paid never said a word they spoke with their actions. Those that hated it made sure we knew it (Dallman 2011).

This is again echoed by designer Daniel Cook (2011b) as well as Playfish studio director Jeferson Valadares at GDC Europe, "People tell you one thing and do another... Sometimes listening to them... it's always important, but it might not be the best thing for a business" (quoted by Caoili 2010b). 
where the gamers do nothing that the designers expect them to do" (as quoted by Elliott 2011b). Metrics address the drawbacks of insular developer culture, as feedback from players drives design rather than relying on what developers think is "cool" or "creative". Developers are fallible. When developers rely on intuition alone, they risk the chance of not giving players what they want. Metrics reduce the risk of game development. As argued by Justin Johnson, "In years gone by it could be pretty hit-and-miss, and you'd have designers that just went on gut instinct. The good ones got it right; but they didn't get it right all of the time - but now you can concentrate on looking at what all of your players want, and work towards providing it for them" (Elliott 2011b). Accordingly, rather than relying on developer insight into player behaviour, metrics are advanced as more reliable replacements for traditional modes of developer intuition.

Quite often, metrics are used to highlight flaws with designers themselves, and fuel discussions on whether more sophisticated metrics can "design out designers", or at least lessen the reliance on unpredictable creative intuition and unreliable predictions of player behaviour. In Casual games, the reliance on metrics comes partly from a belief that developers, especially those migrating from Core, just don't "get it". For example, Henric Suuronen, head of social game studio, Wooga, in an interview with Christian Nutt states:

When it comes to what to think about when designing for social games, I think you have to almost throw everything from your console brain, or your hardcore things, into the garbage. That will get you on the wrong track. Not only is it social, but it's free-to-play. And free-to-play, I think, changes the game design even more than [the fact that] it's social. The free-to-play is a mindset that a lot of console guys don't get (Nutt 2011e).

Lou Castle reiterates this belief, stating that many developers migrating from Core to Casual are unsuccessful because they unable to "put their ego aside and react to what the 
players actually do" (2011). Instead of having creative control over the shape of the game, developers must follow the metrics and statistics about player behaviour to iterate and improve upon the game - to validate (and test) their intuition rather than following it. For Castle, the persons best suited to this form of design are project managers rather than seasoned designers, as the former are already familiar with making data-informed design decisions and focused on delivering predictable (and clearly measurable) results (2011).

In Casual games, game design has already been reduced to a single figure: a Daily Active User/Monthly Active User ratio of .2 or higher, meaning that on any given day at least $20 \%$ of active players are returning to a game (Reader 2010). In Core games, the influence of metrics is not so extreme and most innovations are focused on creating datadriven tools to predict player behaviour based on past behaviours. However, better prediction is commonly equated with less reliance on developers, and thus less risk. For example, achievement data from 14,000 WoW players was used to create models for future actions. As put by one of the system designers, "We are able to predict what a player in a game will do based on his or her previous behaviour, with up to 80 percent accuracy." (Harrison as quoted by Shipman 2011). Ultimately, systems like this can be applied to other gaming formats, or even online retailing, but their allure lies in using data to both govern players more effectively, and reduce reliance on designers: "You could develop a program to steer players to relevant content. Because it is a data-driven modelling approach, it could be done on a grand scale with minimum input from game designers" (Roberts as quoted by Shipman 2011).

Metrics ultimately mean less reliance on developers' intuition and subjective 
views of what is fun, and a greater reliance on data analysts and predictive governance efforts. The Core industry is advised to abandon thinking of game design as a creative art and instead rapidly adapt more "scientific" techniques. For example, Zynga's Erik Bethke advises Bioware about how to reach success with their new Star Wars MMO: ${ }^{188}$ "I think they need to ... get there through science-based metrics...You have all the data in the world about why you are currently failing, and you can correct it in real-time (emphasis added Bethke et al. 2010). Bethke criticizes designers who rely on intuition rather than data to design games, further insinuating that the only way to prevent failure is to embrace data-driven design. The emphasis here is that the Core industry is currently struggling because they treat game design as an art, or even a craft, rather than a science.

A few executives counter this argument with the response that artistry can be productively combined with metrics-driven design. As Alan Miller states, "It's important to realize, we're not selling accountancy software. You're creating an emotional experience, it's an art form, individual style is tremendously important" (as quoted by Stuart 2011). However, still more executives, such as Andy Tian and Lou Castle, believe that game design should be akin to a technical craft rather than an art, with little room for exploration. In all cases, metrics carry the final word because they are incontrovertible: they are precisely measured, quantifiable, and objective. There is room for play in metrics-driven design, but only if this play runs in parallel and not in opposition to what the metrics are 'saying'.

188For a young "upstart" company, Zynga's representatives enjoy inciting controversy, and commonly advise industry veterans about what they're doing wrong. In this case, Bethke was framing Bioware's game as a failure despite the game being nearly 2 years away from launch. 


\subsubsection{Playing the Numbers Game}

With the rise of metrics, game development becomes a numbers game. While metrics are touted to developers and publishers alike as tools to refine game play, ultimately there is a power imbalance in terms of who is interpreting the data, and how this data influences the decision-making process of the interpreter. As put by designer and author Greg Costikyan, the problem with games that rely on metrics, especially social network games, is:

[T]hat they give the suits, if you will, the whip hand. Have a design idea? Try it out! If the metrics say it increases our ARPU, then it's good! Keep it! If not, kill it. (ARPU is "average revenue per user", a telephone-industry term that's been creeping into the discussion of online games recently.)....From the perspective of, say, an aggressive Internet entrepreneur, this is heaven; you don't have to cater to those annoying game design dweebs and their abstruse theories, you just try shit out and if it works you keep it. (Costikyan 2010)

Power rests in the hands of publishers, who have complete financial control over the development process.

While developers believe that a game is an end in itself, publishers have different motives:

... publishers use it as a means to profitability. This makes them a completely different animal: their priorities are different, their expectations from games are different, and their outlook of "success" will also be different most of the time. Not evil, just different in ways that often run counter to the interests of the medium. (Bruno 2010)

Jose Gonzalez Bruno further argues that statistics that are meant to aid developers are taken out of context by publishers, who use them as "proof" of what players really want. Metrics are seen as more reliable predictors of both player behaviour and a game's ultimate success. Accordingly, publishers such as EA are shifting their focus. Andrew 
Wilson, senior VP of worldwide developer at EA Sports elaborates on this change:

It has been a gut-wrenching change for us as an organization, as we move from build-launch-build-launch, to build-launch-live service, forever. No longer is it about the opinion of what the producer or designer thinks is great, now we are a consumer-driven development organization who are constantly changing based on real-time feedback on an hourly basis from the people who play our games (as quoted by Brown 2011, emphasis added).

This further entrenches the risk-averse nature of the game industry, as games become "mathematically tailored experiences, targeting the lowest common denominator with unprecedented precision" (Bruno 2010). Metrics consequently frame design discussions in terms of cutting some features while improving others in order to save time and money. But this appeal to budgets, supported by inarguable numbers from metrics obscures other ways of playing the development game. For example, developers may see the fact that $80 \%$ of players play the male default character merely as a function of a poorly designed menu (i.e. players didn't know they could chose other characters) whereas publishers seize upon the statistic as a rationality for cutting underutilized and expensive player customization options. While both arguments may have merit, the power imbalance between publishers and developers means that "developers will end up losing such arguments more often than not, and we the audience will end up settling for lesser games" (Bruno 2010).

Metrics allow publishers to further rationalize the creation of cultural commodities, reducing the reliance on creative professionals, and thus allowing publishers much more influence over design. As stated by Steve Perlman, founder and former CEO of the cloud gaming service, OnLive, the true benefits of cloud gaming services and online connectivity lie in the ability to stream data back to publishers: "So 
we gave the publishers tons and tons of data, they love it. It allows them to go and make decisions, to design games better" (emphasis added, Martin 2011a). The wording of this last quote is important. Metrics and telemetry allow publishers - not developers - to make key design decisions. As the above quote also demonstrates, the phasing out of creative autonomy is smoothed over with references to increased reliance on users. However, crowdsourcing creativity through the use of metrics is a difficult endeavour.

Metrics - even real-time metrics - are fundamentally retrospective. They indicate what mechanics are currently popular, and can refine and perfect existing mechanics, but in doing so they contribute to repetitive and self-referential game design. As put by indie developer Edmund McMillen, developer of Super Meat Boy:

The funny thing about [metrics] and business in general is the idea that they think they're perfecting something and they're going to be more successful by perfecting it. When in reality, I guarantee you something will come out in the next few years that will beat out these games, and it will be something nobody knows about, and something nobody knows they wanted, because the thing that people really want is something they don't know exists. (as quoted by Sinclair 2010)

This sentiment echos arguments made by Saul Greenberg and Bill Buxton about usability studies in general (2008). The reliance on these forms of evaluation encourages developers to solve problems through iterative refinement, following a life cycle of "design, implement, evaluate". But this leads to local hill climbing (minute improvements along similar lines until an optimum state is found) at the expense of considering and developing much better ideas that lie further afield, far beyond the purview of simple $a / b$ tests.

As the Core industry has long known (Kerr 2006), using current user feedback to 
learn about current customers tells you nothing about how to innovate or break into new markets. And while metrics are continually evolving in order to provide contextual cues about player behaviour (i.e. what were they doing just before they quit, or just before they purchased an item), there are many things metrics cannot tell developers (i.e. what motivates player behaviours).

Many of these aforementioned problems are addressed through the increasing surveillance of players, collecting more intimate and refined knowledge about what players are doing in-game, and linking this information to what players are doing outside of the game (e.g. demographic details, larger purchasing and consumption habits, social networks, etc.).

However, there is a larger issue at stake as metrics promote the increasing rationalization of play (or at least the increasing rationalization of the design of play spaces). This rationalization is based on computational logics that are inherently flawed that metrics are objective and that they can effectively measure 'fun' - when in fact, developers and publishers exploit these systems as much as players develop exploits for games (perhaps even more so, given that the effective gaming of metrics can make the difference between attracting both investors and players versus becoming just another failed games company) .

While the statistics derived from telemetry are seemingly objective, small changes to how these metrics are interpreted, visualized, and aggregated can drastically change the outlook on a game, transforming it from a vaunted success to a sinking ship. For example, when Facebook revised its method for counting users in October 2011, Zynga 
dropped from 262.5 million aggregated monthly active users to 195.5 million a day later. This one small change equated to a loss of $25 \%$ of users (Orland 2011a). At the time, Zynga was preparing for its IPO and this drastic drop negatively effected its ultimate valuation.

Another example of the shaky objectivity of metrics is when publishers purposely "game" these measurements in order to attract investors. In this sense, instrumental play is at work. The development game is less about creating or exploring, but more about developing an efficient strategy to make the numbers lead to success, even if this means exploiting the data.

Exploiting metrics is a commonly recognized practice in the industry. Trip Hawkins, founder of EA and Casual games company Digital Chocolate, provides a detailed analysis of how Monthly Active Users (MAU) for social games are artificially inflated by advertised installs, where players are incentivized to install a new game in order to earn virtual currency for a game they are currently playing, "MAU are very misleading...Two games can have the same MAU but one is really a success and the other is really a failure (2010). What is interesting in this case is that while Hawkins acknowledges that metrics are easily manipulated, especially by developers to whom "exploits" and "gaming" the system are deeply ingrained cultural tropes, he maintains the belief that these machinations may be exposed with the collection and publication of even further metrics. In other words, despite their current failures, Hawkins believes that metrics can be objective. ${ }^{189}$

189Introducing more sophisticated technology in the face of dramatic failures in surveillance is a theme not restricted to the game industry. Paul N. Edwards' history of control technologies during the Cold War is a particularly illuminating example of this and the associated discourse that everything in the world can be measured and predicted (1996). 
This provides an example of how the instrumentalization of play works in counter-operative directions. While play spaces seem to be increasingly rationalized, constrained, and governed via metrics, at the level of the game industry, there are multiple examples of developers "playing" with metrics, reflexively learning about the systems and the structures of the metrics game, uncovering the rules of which metrics to measure, and which metrics determine "winning conditions" for the game (e.g. a high MAU/DAU ratio signals success to investors) and then playing with different collection methods, calculations, and visualizations to discover an optimal strategy.

Numerous examples of gaming the metrics system are found in the mobile and console downloadable markets, where developers struggle for visibility amidst the multitude of other games. Generally, games with the highest number of downloads are selected for promotion by the platform operator, such as the ubiquitous "top downloads" and "top sellers" lists in the Apple App Store, XBLA, Steam Store, Google Play, etc. ${ }^{190}$ Strategies for "gaming" the metrics of a game's success are myriad. For example, on the Steam Store, the continual release of DLC for a game ensures visibility under the "new releases" section on the homepage. In the mobile markets, developers play with strategies to game the leaderboards by inflating download numbers. Visibility is ensured by number of downloads, and those games with the highest number of installs ${ }^{191}$ are guaranteed front page visibility. This visibility and promotion thus ensures more downloads in a selfreinforcing cycle. Not surprisingly, one of the most rudimentary methods to game

190 "Gaming the blade" was a prominent feature in my early developer interviews, as local developers experimented with strategies to increase their visibility on the Xbox 360 dashboard ("the blade"). 191This itself is an artifact of console and PC games, where number of installs directly equated to the revenues for a game. However, in the Casual games market, the relationship between installs and profits is much more nebulous. 
leaderboards is to offer the game for free, either as a F2P game or as a free demo. Another method is "pay-per-install incentives" where one game offers virtual currency if users download or buy another app from the same developer (or a partner developer).

Platform owners play an escalating game of their own, attempting to discover and shut down these exploits. For example, the Apple App store banned pay-per-install incentives in the summer of 2011 (Crossley 2011g). Google's Android Market also changed their metrics algorithms in the summer of 2011 to subvert the artificial inflation in popularity due to advertising. ${ }^{192}$ Google refused to comment on the extent of the changes "because doing so might invite manipulation" (Crossley 2011h). Apparently, acknowledging that metrics are fallible could incite further tampering.

Even subjective criticisms of games are now quantified, and in turn, gamed. Metacritic.com translates reviews from critics and users into percentages, and aggregates all reviews of a game (or movie, or TV show) into two scores: one score aggregating critic reviews and one score aggregating user reviews (for example, Skyrim for PS3 received a critic score of $92 / 100$, and user score of 5.3/10). Widely used by consumers to determine whether a game is worth purchasing, the site is also employed by publishers to gauge the overall success of a game and distribute royalties to developers (Totilo 2008). Publishers rely on metrics as they simply do not have the time (or the skill) to play and judge the games for themselves (S. Johnson 2009). However, blogger Jose R. Gonzalez Bruno extends this argument, stating that executives are numbers people (2010). Thus they respect Metacritic because it reduces many subjective, qualitative opinions into a

192The day these changes were made, one developer found his app jump to 11th from 63rd in the rankings of top free social apps. He speculates on the changes: "It's a fairly gameable system. The whole point of an ad spend is to drive you to No. 1 in the rankings. That may still happen but it's nice if the rankings are not as gameable" (Geoff Cook as quoted by Crossley 2011h). 
single number with the illusion of objectivity. Not surprisingly, Metacritic continually faces accusations that scores are gamed, particularly by developers who masquerade as regular players in order to inflate user scores (M. Fahey 2011). This is just one more example of how 'success' in the game industry is rooted in objective numbers, numbers that are continually gamed.

The Metacritic example illustrates that numbers are innately convincing, not just to publishers by also to developers and players. Developers have difficulty resisting the Casual Revolution, the growth of metrics, and all of the negative implications these may have for their continued creative autonomy, because they believe that - despite exploits here and there - data is unassailable. They are at heart power gamers focused on efficiency, achieving goals, and levelling up. The feedback the development game is giving them (measured by player numbers and profit margins), is that in order to succeed, they must embrace at least some aspects of metrics and metrics driven design, even if it means that many designers are effectively designed-out of game design.

\subsection{Conclusion}

Initially resisted by Core developers, metrics are increasingly accepted as the cost of remaining in business. While publishers and studio heads emphasize the economic potential of metrics (e.g. opening new revenue streams and streamlining production processes), developers emphasize very different benefits. Development becomes a godgame, learning about player behaviours and how to shape them. The allure of designing games as governance tools accordingly attracts developers and publishers alike. 
However, with the growth of metrics game design becomes less about creativity, and more about gaming the numbers: harnessing, massaging, and exploiting data steams.

The examples in this chapter can be applied to other creative workplaces outside of the game industry by relating them to the New Spirit of Capitalism. While the original Spirit of Capitalism proposed by Boltanski and Chiapello in 1999 relied on visionaries and great men to tie networked projects together and give them direction, the Spirit of Capitalism seen in the game industry today replaces fallible, subjective visions with data gleaned from the masses. The reliance on metrics addresses many of the original shortcomings of the Projective Cité, as better ways to predict complexly shifting networks of consumers directly relates to job security, and rather than being constrained by management hierarchies, developers are given more autonomy in their work. However, the creativity that was emphasized by earlier versions of the New Spirit of Capitalism is tightly constrained by this self-same data. Creativity does not lie in a single developer's intuition, but in finding patterns in the data from crowds of dividuals and harnessing them. This reliance on data re-establishes the rationalization that was rejected by the Second Spirit of Capital, but re-formats it into a mode that is much harder to critique because the data is coming from the ground-up (populations of users) rather than handed top-down by bureaucrats. 


\section{Chapter 11. End Game}

The game industry is an industry predicated upon play. If any industry should emphasize intuition, creativity, and subjectivity, you might think it would be game development. Yet, here it is, increasingly rationalized, automated, and driven by numbers. On a cultural level, what does it mean that our most popular games are premised on hyper-efficiency and even the time we spend in seemingly idle play is surveilled and commodified?

The thesis provides an explanation of massive shifts in the ways games are both made and played. A few short years ago, Casual sectors burst upon the scene. Their success - especially the success of Facebook - was unpredicted, and their rising popularity - both in terms of player numbers and profit margins - was inexplicable to most Core developers, who responded with vociferous criticisms. Yet, a few short years later many of the loudest critics are now making these very same games (e.g. Soren Johnson and Ian Bogost) and numerous icons of Core have defected to Casual (e.g. John Romero, Sid Meier, Brian Reynolds, Brenda Brathwaite). Metrics are designing out designers, replacing creative intuition with predictive governance. How did this all happen? And how do shifts in the game industry reflect larger shifts in the operation of capitalist systems?

\subsection{Retracing Our Steps}

In this thesis I argued that game industry shifts are a way to examine how the New Spirit of Capitalism operates on the ground. Capitalism absorbs and adapts to critique by altering its material forms and underlying systems of order. These adaptations explain 
many of the current upheavals in the game industry, as Core console production models decline and new Casual sectors and gamification efforts rapidly take their place. The shifts in dominant game sectors can be visualized in terms of the Industrial Cité and the production methods it espouses slowly being replaced by network-centric methods of production premised on the Projective Cité.

The New Spirit of Capitalism legitimates itself in terms of rationalities of selfgovernance and autonomy, initially sacrificing the material and psychological security that was prioritized by the Second Spirit of Capitalism in exchange for freedom from hierarchies. New adaptations to the Spirit of Capitalism based on metrics and predictive governance now offer the security that was absent in the original New Spirit of Capitalism. This is achieved by introducing novel, less objectionable, forms of rationalization based on increasingly precise consumer data. While limiting the forms of creativity that developers are able to express, these metrics allow capital to colonize more and more of our everyday experiences. From the emotions and behaviours we exhibit during game play, to our intimate real world experiences via gamification, all are subjected to predictive governance processes.

I began this thesis with gamification, showing how it is the idealized version of the New Spirit of Capitalism. I argued that the forms of surveillance and feedback in games have been adapted and applied to our non-game experiences - from our social relationships, to our school and workplaces, to our health and fitness, and beyond. I intentionally started at the end of the story in order to set the stage for the remainder of the thesis, which traced how games and play became such powerful tools of governance. 
At the end of the third chapter, I asked: How did it come to be that we effortlessly accept play as productive? How can games move so seamlessly from sandbox to office space?

In Chapter Four, I started answering these questions, showing how the instrumentalization of play fuels an ideological shift that allows the New Spirit of Capitalism to take root. Instrumental play focuses on advancement within the game. Play becomes a means to an end, rather than an end in itself. It is goal directed and centred on rewards. The instrumentalization of play is what allows the shift in the way we treat play. Play changes from something that is idle fun to something that can be made productive. The instrumentalization of play is not new. It began long before the first digital games, when sport was first professionalized and a strict adherence to standardized rules was required in order to commodify the experience (see Grimes and Feenberg 2009; O'Donnell 2008). What is new, however, is its reach. More and more digital games cater to hyper-efficient power gamers and fewer games emphasize ludic, exploratory play styles. Other important transformations are happening as playful systems of social rationality and game-modes start to overlay everyday tasks, as happens with gamification. The boundaries between work and play, game and reality, blur and disintegrate further. Games are now productive.

In order to understand this phenomena, we must look at how games are made. I traced the rise and fall of game industry in order to develop a picture of how game development works, what it looks like at ground level, and where its future lies. In Chapter Five, I addressed the relative paucity of developer-centric research and outlined my research methodology. In Chapter Six, I began my exploration of game development 
by starting at the bedrock of the industry: Core console development. I argued that traditional models of production in the game industry are hierarchical, unwieldy, and exploitative. They turn developers - the creative force behind game development - into just another cog in a massive, rationalized, risk averse system. However, as Chapter Seven showed, developers are not passive dupes. The instrumentalization of play becomes a discourse that sensitizes developers and players into these new forms of games as capitalist tools of governance. What they see in games and gamification coincides with their moral experience of daily life (i.e. the grind of goal-focused, hyper-efficient power gamers) and suggests models of action they can grasp. Despite undesirable working conditions, Core culture and developer communities of practice premised on instrumental play perpetuate these flawed production systems. However, increasing creative constraints on Core production have pressured the game industry to innovate in order to survive.

In Chapter Eight, I panned out from the Core console sector, showing the larger landscape of game development. This chapter emphasized how the New Spirit of Capitalism innovates following critiques, thus revitalizing and transforming traditional game sectors such as MMOs, PC, and even Console. These innovations encourage more Projective modes of production, promising agility and autonomy instead of the hierarchies and risk aversion of the Console Sector. Chapter Nine continued in this line, emphasizing emerging social, mobile, and Casual sectors that exemplify the Projective Cité. While individual developers resist to these emerging sectors, they cannot justify this resistance in terms of the accepted ideological language of the New Spirit of Capitalism. 
Developer resistance to new sectors falls flat partly because these innovations align so closely with instrumental play, a valuation on numbers, and goal-orientated achievement - regardless of the means. And, when the customer is king, it is metrics and not developers, who are authorized to speak.

Chapter Ten situated industry wide shifts in the game industry within the growing reliance on data-driven design. As each game sector innovates, it becomes more and more dependent upon metrics, as seen in Illustration 11.1, below. This illustration emphasizes how game development becomes more centred on predictive governance - anticipating and then shaping player behaviour.

Illustration 11.1: Data and predictive governance in the game industry Each layer integrates the uses of previous layers

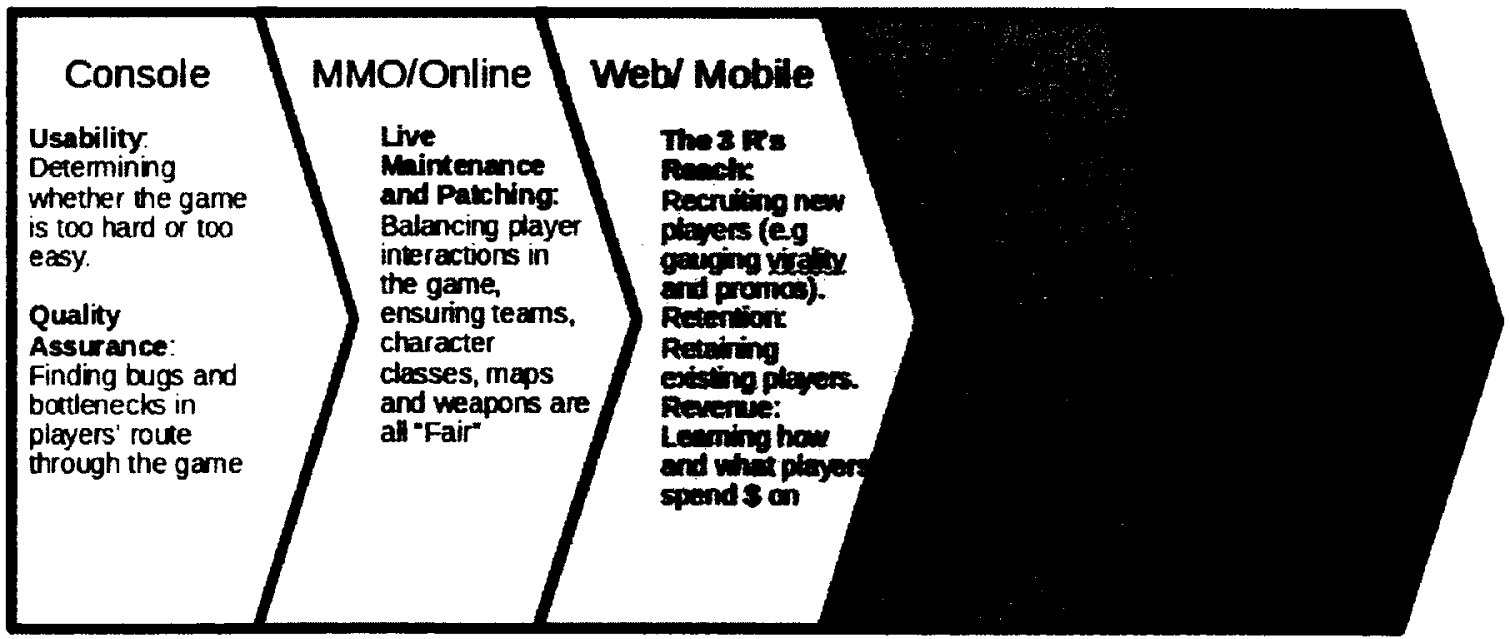

\section{Reliance on metrics increases with each layer}


While rationalized in terms of economic efficiency, this dependence on metricsdriven design challenges developers' own autonomy as the creative force behind games, reclassifying their work from an art to a statistical science and thus rationalizing game design. Metrics-driven design facilitates both the valuation of dividuals over individual players, as well as the "designing out of designers". It rejects the idealized creative freedom that characterizes design, deeming it wasteful and inefficient.

\subsection{What This Thesis Means for Game Development and Beyond}

This leaves us in a rather dark place. Metrics are predicated upon predictive governance watching what we do, in the hopes of shaping how we behave. In terms of the operation of governance, metrics replace man with the machine. Yet, because this machine is created directly from crowdsourced user data, this control seems to come from the bottom-up. And despite the inherent flaws with metrics (i.e. they ignore subjective context and are easily exploited), the posited solution in the game industry is not to avoid metrics, but rather to increase their precision.

The findings of this thesis are not restricted to the game industry alone, but can be extrapolated to other workplaces. This thesis has shown how capitalism continually adapts its form, paralleling shifts in the underlying value systems of the populace. In this context, studying the game industry is important because it adds a corrective to the original Spirit of Capitalism proposed by Boltanski and Chiapello in 1999, showing how technology - by emphasizing quantitative forms of reasoning and rationalization - plays an incontrovertible role in capitalism. Technology, particularly data collection technology, 
is easily framed and created to promote productivity and accumulation. Even creativity can be automated and made more efficient, and the emergent complexity of networks can be distilled down and harnessed into actionable data. In games and spaces of play, the influence of technology has promoted this form of rationalization, fuelling the growth of instrumental play. In gamification and beyond, this rationalization is hidden behind discourses of 'play' and 'fun', despite the fact that, in actual practice, both of these attributes may be completely absent.

The central lesson of this thesis is that the adaptations of capitalism continually silence critique. For example, in the game industry, while emerging sectors alienated many Core developers, they also offered solutions to many of the problems of traditional development (i.e. long production schedules, massive teams, risk aversion, struggles for publisher support). The new revenue streams that Casual sectors popularized promised a freedom from the economic constraints that have traditionally stifled creativity. Agile, Casual production models premised on the Projective Cité, foregrounded flexibility, innovation and proficient, self organized networks over the slow lumbering hierarchies of Core. They emphasized information as source of productivity and profit and promise a better way to communicate with, and thus serve, players.

More broadly, metrics are sold as the route towards salvation for struggling industries. Data technologies are a way to escape economic constraints, substituting selfcontrol and autonomy in place of tyrannical management. Metrics thus structure and support new networked modes of production. Instead of relying on visionaries and creative directors to determine what consumers want, metrics point the way forward, 
driving design. However, this liberation from old oppressive mechanisms of capitalism comes with new mechanisms of oppression - the eventual valuation of quantitative data over subjective, and thus fallible, individual human creativity.

\subsection{Ways Forward}

The game industry, while illustrating the dangers of an over-reliance on metrics, can also offer ways to mediate these dangers. There are routes forward and ways to lessen the detrimental impacts of data-driven design as well as other negative aspects of the Projective Cité. The solution lies not in rejecting the Projective Cité altogether, but in shedding light on the dark corners that provide spaces for exploitative practices to flourish. With critique, the Projective Cité can be re-made.

Accordingly, the point of this thesis is to make more transparent the debate about the use of metrics in the game industry, in order to tease out and question the underlying value placed on instrumental play, including its emphasis on efficiency, quantification, and winning by any means necessary, and how this feeds into and reinforces the use of games as tools of governance and commodification. This debate is already percolating in the industry itself, thus influencing how metrics are taken up and adapted.

Most developers frame resistance to metrics of a Us vs Them battle (i.e. people who use metrics vs people who do not) (Brathwaite 2011b), but there is a growing realization that this battle is more of a dialectic, as metrics practices filter through the sectors of game design and become an unavoidable way of doing business. The acknowledgement that the use of business-centric metrics is not restricted to outsiders 
and Casual games initiates much larger discussions of how 'real' designers are complicit in unethical design, continually compromising their ethics as the behest of executives, publishers, and managers (Birke 2011). Metrics are seductive. They can be used to drive compulsive behaviours, reshaping spaces of play into spaces of governance and consumption. But they are also useful tools that can be wielded to make games better more social, more emotional, more fun, more educational.

Accordingly, developers are now struggling to cultivate a reflexivity about how metrics are used in the game industry, to determine the rules and boundaries that govern how metrics are utilized, and to ultimately determine how to play with, experiment, and reshape metrics from tools of governance to tools for exploration and play as well. Part of this challenge lies in overcoming pulls of computational logic and the allure of metricsdriven design. Overcoming, in this sense, does not mean rejecting metrics outright, but rather influencing the design and deployment of analytical systems and consciously directing the role they have in shaping the game development industry. While metrics certainly drive a rationalization and instrumentalization of play, there is still space to be playful - to push against the boundaries of the system and reshape the game via more reflexive design.

Not surprisingly, developers are exploring ways to remake, reshape, and exploit the development game in order to general new winning conditions:

Design your ethics into how games will interact with players. Sometimes it's okay to make something fun and compelling. Other times you'll be forced to make concessions. I've done some pretty shameful things in development. I've compromised on principles of violence against women, I've modelled munitions for the army, and I've studied very hard at how to make people keep doing things compulsively when they otherwise wouldn't. (Birke 2011) 
By following such instructions, developers may be able to re-make the metrics game as well. The ethical divide is not between who uses metrics and who do not, as the new economics of game development - and the Projective Cité itself - are now inextricably dependent upon metrics. The divide is now between those developers who design ethics into their games to protect players as well as developers' own creative autonomy, and those who ignore the fact that game design is now more than ever an ethical practice that has real world effects. It is no longer a game.

Developers and players are already remaking the metrics game and reshaping how their industry adopts Projective mentalities. They are voting with their feet. Both players and developers are currently abandoning social game giant Zynga, frustrated with the lack of developer autonomy and the dearth of creative game play (Caoili 2012). They do not abandon metrics altogether, but instead, look to preserve the autonomy, creativity, and spaces of free play that metrics threaten.

Some models of game development - especially those emerging in 2012 after the data-gathering phase of this research was completed - have started to ameliorate many of the drawbacks of game development in the Projective Cité. For example, services such as KickStarter use crowdsourcing from fans to provide development funds, granting developers freedom from publisher oversight and a space to make 'risky' decisions. In this sense, networks can contribute to design innovations rather than "design by numbers".

Changes to the Spirit of Capitalism have initiated tectonic shifts, reforming the geography of the game industry and creating fissures in the landscape that allow new game sectors to emerge. But this landscape is continually re-forming. While capitalism 
continually adapts to silence critique, this means that critique itself must always adapt. It must recognize the Spirits that shape our world, and take up the language of Cités in order to have a voice in dialectical change. Critics, such as myself, must recognize the power of the "network" as a concept that not only shapes academic thought (from SNA to ANT to Empire), but must also recognize how these concepts ultimately influence citizens' everyday ideals and actions, such as how businesses, such as game studios, are optimally run. This is a dialectical game that is never over. 
Appendix A 


\section{Appendix A. $\quad$ Ethics Clearance Form}

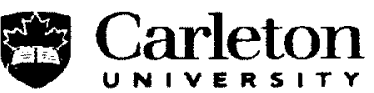

Canada's Capital University

\author{
Carfeton University Research Office \\ Research Ethics Board \\ $5^{\text {tr }}$ Floor Tory building \\ 1125 Colone: By Drive \\ Ottawa. ON K1S SB6 Canada \\ Tel: $613-520-2517$ \\ Fax: $613-520-2521$ \\ ethics.
}

\section{Ethics Clearance Form}

This is to certify that the Carleton University Research Ethics Board has examined the application for ethical clearance. The REB found the research project to meet appropriate ethical standards as outlined in the Tri-Council Policy Statement: Ethical Conduct for Research Involving Humans, $2^{\text {no }}$ edition and, the Carleton University Policies and Procedures for the Ethical Conduct of Research.

- New clearance

X Renewal of original clearance

Original date of clearance: 2 September 2009

Date of renewal

Researcher

Status

Supervisor

Funding status

Project number

Title of project

31 May 2011

Jennifer Whitson

Ph.D. student, Sociology \& Anthropology

Professor Aaron Doyle, Sociology \& Anthropology

Non-funded

12-0046

Gaming the system: Emergence, governance and control in games

Clearance expires: 31 May 2013

\section{All researchers are governed by the following conditions:}

Annual Status Report: You are required to submit an Annual Status Report to either renew clearance or close the file. Failure to submit the Annual Status Report will result in the immediate suspension of the project. Funded projects will have accounts suspended until the report is submitted and approved.

Changes to the project: Any changes to the project must be submitted to the Carleton University Research Ethics Board for approval. All changes must be approved prior to the cantinuance of the research.

Adverse events: Should any participant suffer adversely from their participation in the project you are required to report the matter to the Carleton University Research Ethics Board. You must submit a written record of the event and indicate what steps you have taken to resolve the situation.

Suspension or termination of clearance: Failure to conduct the research in accordance with the principles of the Tri-Council Policy Statement: Ethical Conduct for Research Involving Humans $2^{\text {nd }}$ edition and the Carleton University Policies and Procedures for the Ethical Conduct of Research may result in the suspension or termination of the research project.

Antono K Guatiert

Antonio R. Gualtieri, Chair

Carleton University Research Ethics Board 
Appendix B 


\section{Appendix B. Interview Question Guide}

1) Tell me about yourself (prompts: age, educational background, work history, position, favourite games)

2) Tell me about your work (prompts: what does a typical day look like?)

3) What are you currently working on? (prompts: ask for a description of the game, the timeline)

4) Do you like your work? (prompts: if you could change anything about your work, what would it be?)

5) Do you ever have crunch periods?

6) What's your team like? (prompts: who do you work with?)

7) How's your company organized? (prompts: how many employees, teams? what different games are in production? how are teams composed? is there a management hierarchy? who do you sit near?)

8) How do you decide what game to make?

9) What tools/engines are you using?

10) What problems do you run into during development?

11) How do you learn about players?

12) What feedback do you get from players?

13) How do you ensure that players are playing the game as intended?

14) Do players ever do anything unexpected?

15) How do you fund a game's production?

16) What's the economic model of the game? (prompts: ask about game sales, ask 
about production costs versus revenue)

17) What's working with (insert publisher, manufacturer) like? (prompts: ask about what feedback is given, how certification is going, how easy it is to patch or localize, etc.)

18) How do you maintain the game after launch?

19) Where do you see the game industry going?

20) Is your company working on anything new? (prompts: next-gen tech, a shift to a different segment, etc.)

21) What would you change about the industry? 
Appendix C 


\section{Appendix C. $\quad$ List and Description of Online Sources}

\section{Description of Online Sources}

Unless otherwise noted, I started following these sources as of August 24, 2009. My analysis continued until December 31, 2011 (approximately 2 years, 4 months). I have organized my online sources according to topical category.

Industry News Sites

\section{- Gamasutra (www.gamasutra.com)}

- Founded in 1997, Gamasutra is the online sister publication to Game Developer Magazine. It is composed of five sections: News, Features, Developer Blogs, Job Listings, and Contractor Listings. Each article is tagged according to category (Programming, Art, Audio, Design, Production, Biz/Marketing) and sector (All, Console/PC, Social/Online, Smartphone/Tablet, Independent, Serious).

- GameSetWatch (www.gamesetwatch.com)

- Founded in November 2005, GameSetWatch was a sister site of Gamasutra. It provided "alt.game" blogging and news highlighting little known independent games. It ended production in November of 2011, after six years of reporting. I followed GameSetWatch from May 2010 - November 2011.

- Inside Social Games (www.insidesocialgames.com)

- Started in April 2008, Inside Social Games provides news and market research exclusively about Facebook and social gaming ecosystem. Its services include interviews, news articles, as well as detailed statistics and industry growth analyses. It also offers in-depth research reports. It is a part of the "Inside Network" a market research organization. I started following Inside Social Games May 2010.

- Worlds in Motion.Biz (www.worldsinmotion.biz)

- Launched in July 2007 by Game Developer Research, Worlds in Motion provided analysis, statistics and news about social and online gaming, particularly MMOs and virtual worlds. It was amalgamated with Gamasutra and added to its Social \& Online Games section in 2011. I started following Worlds in Motion May 2010.

- GamesIndustry.biz (www.gamesindustry.biz)

- Launched in May 2008 by Eurogamer, GamesIndustry.biz provide news and information about the global videogame industry, and focuses on businessfocused news, opinion articles, interviews, and analysis. I started following GamesIndustry.biz in May 2010.

- Industry Gamers (www.industrygamers.com)

- Industry Gamers provides business-focused news and information about the global game industry, including interviews, analysis, and statistics about market trends. It amalgamated with gamesindustry.biz in 2012. I started following Industry Gamers in May 2010. 
- Develop (www.develop-online.net)

- Active since July 2007, Develop Online is the online sister site for Develop magazine. Based in the UK, it is aimed exclusively at the development community. It is composed of sections for News, Feature Articles, Developer Blogs, Event Listings, and Job Postings. I started following Develop in May 2010

Player News Sites

- Kotaku (www.kotaku.com)

- Part of Gawker Media's network of sites, Kotaku provides gamers with news about the game industry and anything broadly related to gamer culture. It includes forums for user discussion.

- Tap-Repeatedly (www.tap-repeatedly.com)

- Aimed at game players, Tap-Repeatedly provides feature articles, editorials, news about the industry, and reviews of games. I started following TapRepeatedly in May 2010.

General Technology News Sites

- Slashdot (www.slashdot.org)

- Founded in 1997, Slashdot is a technology-related news website owned by Geeknet, Inc. It features user-submitted current affairs news stories about science and technology related topics. Each topic is open to discussion by readers, who rank each others' comments in a user-based moderation system.

- TechCrunch (www.techcrunch.com)

- Founded in 2005 by Michael Arrington, TechCrunch provides profiles of startup companies, products and websites, alongside technology news and analysis. It was acquired by AOL in 2010.

Blogs and News Sites founded by Industry Consultants

- Game Theory (www.gametheoryonline.com)

- Run by Scott Steinberg, CEO of TechSavvy Global (a strategic consulting and consumer products testing firm), Game Theory provides videos and articles about the game industry. I started following Game Theory in June 2010.

- GAMESBrief (www.gamesbrief.com)

- Written by Nicholas Lovell, a consultant on financial and strategic advice for games companies, GAMESBrief is a blog dedicated to the business of games. I started following GAMESBrief in July 2010, after reading Lovell's articles in Develop and Gamasutra.

- Gabe Zichermann - The Gamification Blog (www.gamification.co)

- Founded by Gabe Zichermann to promote gamification, The Gamification Blog provides case studies of gamification, and interviews with designers of gamification services. I started following The Gamification Blog in August 2010 . 
- What Games Are (www.whatgamesare.com)

- A blog about game design, publishing, marketing and production, What Games Are is written by Tadhg Kelly. Kelly has worked in the games industry for twenty years as a designer, writer, producer, and startup founder. I started reading What Games Are in December 2010 after reading his articles in Gamasutra.

Personal Blogs of developers and developer-academics who reflect on their work processes.

- $\quad$ Pippin Barr (www.pippinbarr.com)

- Barr reflects on developing indie games (The Artist is Present, Epic Sax Game, Let's Play:Ancient Greek Punishment) as well as his experiences playing AAA games. Barr works at the University of Malta.

- Games for Social Networks (www.games4networks.posterous.com)

- Aki Järvinen writes about developing social games. He works for Digital Chocolate, a social games company, as Creative Director. I started following Järvinen in March 2010.

- Gamepocalypse Now (www.gamepocalypsenoew.blogspot.ca)

- Jesse Schell is the CEO of Schell Games and a professor at Carnegie Mellon. His blog provides examples of gamification as well as his reflections on the movement. I started following Gamepocalypse Now in March 2010.

- Digital Chocolate (www.blog.digitalchocolate.com)

- Trip Hawkins, founder of Electronic Arts, started this blog in 2009 to reflect on the growth and design social games, especially those games created by Digital Chocolate, his new startup. In 2012, Hawkins stepped down as CEO of Digital Chocolate. I started following the blog in March 2010.

- Designer Notes (www.designer-notes.com)

- Soren Johnson is a game designer and programmer. He was a once-vocal critic of social games who shifted employment from EA to Zynga in 2011. He has been writing Designer Notes since 2005. I started following Designer Notes in March 2010.

- Applied Game Design (www.bbrathwaite.wordpress.com)

- Brenda Brathwaite is an industry veteran who started designing RPG games in the early 1980's. She co-founded the social game company Loot Drop with John Romero in November 2010. I started following Applied Game Design in May 2010.

- Shambling Rambling Babbling (www.caseyodonnell.org/blog)

- Casey O'Donnell is a professor at the University of Georgia, who previously worked in the game industry. O'Donnell writes anthropological accounts of game developers in the United States and India. I started following Shambling Rambling Babbling in July 2010.

- Raph Koster's Website (www.raphkoster.com)

- Koster is a MMO game designer and author of A Theory of Fun. He started his 
website in 1998. I began following Koster's blog in July 2010.

- Videogame Theory, Criticism, Design (www.bogost.com)

- Ian Bogost is a professor at Georgia Tech, game designer, and author of multiple books on game studies. I started following Bogost's blog in July 2010.

- Lost Garden (www.lostgarden.com)

- Daniel Cook is a game designers and artist, who has been working in the industry for over 15 years. His blog, started in 2005, features article-length essays on the design process. He is currently the Chief Creative Officer at Spry Fox. I started following Lost Garden in January 2010.

- Chris Hecker's Website (www.chrishecker.com)

- Chris Hecker is a game developer and industry speaker, who previously worked with EA Maxis on Spore. He is the founder of the Indie Game Jam, and a frequent speaker at game developer conferences. I started following Hecker's blog in June 2011.

- Sexy Videogameland (www.sexyvideogameland.blogspot.ca)

- Sexy Videogameland is the blog of games journalist Leigh Alexander. Alexander writes for Gamasutra, Kotaku, Edge, and Nylon. I started following Sexy Videogameland in June 2011.

- The Game Prodigy (www.thegameprodigy.com)

- A collection of articles about game design and development written by Brice Morrison. The Game Prodigy finished posting in March, 2011.

Printed Sources

- Game Developer Magazine

- Aimed at working and aspiring game developers, Game Developer's first issue was launched in March 1994. The magazine publishes articles relating to programming, art, audio, quality control, design and production. Each edition contains at least one postmortem overview of a game's development and launch. With my subscription, I accessed and downloaded all issues from May 2004 to December 2011. I read and coded each postmortem for those years.

- Wired

- Published since 1993, Wired reports on how new and developing technology affects culture, the economy, and politics. Its techno-utopian outlook (and Apple fan-boyism) can be attributed to the influence of Stewart Brand, Nicholas Negropointe, Chris Anderson, and Kevin Kelly. I started subscribing to Wired in July 2010. 
Additional sources

I relied on additional sources to supplement my data collection. I periodically drew from the following sites, but did not follow them on a systematic basis.

- Gaming News Sources:

- Edge (www.edge-online.com)

- Rock Paper Shotgun (www.rockpapershotgun.com)

- The Escapist (www.escapistmagazine.com)

- IGN (www.ign.com)

- Casual Gaming.biz (www.casualgaming.biz, now amalgamated by www.develop-online.net)

- Inside Mobile Apps (www.insidemobileapps.com)

- GamePro (www.gamepro.com, now amalgamated with PCWorld.com)

- Gamezebo (www.gamezebo.com)

- Gamespot (www.gamespot.com)

- Game Informer (www.gameinformer.com)

- Gaming Business Review (www.gamingbusinessreview.com)

- Joystiq (www.joystiq.com)

- PC Gamer (www.pcgamer.com)

- Games Radar (www.gamesradar.com)

- Inside Facebook (www.insidefacebook.com)

- All Facebook (www.allfacebook.com)

- Tech Industry News Sources

- Ars Technica (www.arstechnica.com)

- Venture Beat (www.venturebeat.com)

- Forbes (www.forbes.com)

- Time (www.time.com)

- New York Times (www.newyorktimes.com)

- Guardian UK (www.guardian.co.uk)

- Wall Street Journal (www.wsj.com)

- Fortune (www.money.cnn.com/magazines/fortune)

- Huffington Post (www.huffingtonpost.com) 
Appendix D 


\section{Bibliography of Primary Data Only ${ }^{193}$}

Acton, Mike. 2011. "Game Dev Doesn't Have to Suck.” Develop. Retrieved January 19, 2011 (http://www.develop-online.net/news/36785/OPINION-Game-developmentdoesnt-have-to-suck).

Adams, Ernest. 2010. “The Designer's Notebook: Selling Hate and Humiliation.” Gamasutra. Retrieved May 10, 2010

(http://www.gamasutra.com/view/feature/4319/the_designers_notebook_selling_. php?print=1).

Alexander, Leigh. 2011. "Balancing Metrics And Creativity Key To Item Sales In The Sims Social." Gamasutra. Retrieved October 17, 2011

(http://www.gamasutra.com/view/news/37799/gdc_online_balancing_metrics_and -.php).

Alexander, Leigh. 2010a. "Game Violence, Real Aggression Don't Correlate In Aussie R18 Debate." Gamasutra. Retrieved December 1, 2010 (http://www.gamasutra.com/view/news/31797/Game_Violence_Real_Aggression _Dont_Correlate_In_Aussie_R18_Debate.php?

utm_source $=$ feedburner\&utm_medium $=$ feed\&utm_campaign $=$ Feed $\% 3 \mathrm{~A}+$ GamasutraNews+\%28Gamasutra+News\%29).

Alexander, Leigh. 2010b. "In An Era Of 'Anguish', Game Design Searches For Its Soul.” Gamasutra. Retrieved October 13, 2010 (http://www.gamasutra.com/view/news/30587/Analysis_In_An_Era_Of_Anguish _Game_Design_Searches_For_Its_Soul_.php).

Alexander, Leigh. 2010c. "Reynolds Pioneers For Zynga With New FrontierVille." Gamasutra. Retrieved June 10, 2010 (http://www.gamasutra.com/view/news/28872/Interview_Reynolds_Pioneers_For _Zynga_With_New_FrontierVille.php).

Alexander, Leigh. 2010d. "Video Games Keep Tricking Us Into Doing Things We Loathe." Kotaku. Retrieved November 4, 2010 (http://kotaku.com/5678356/videogames-can-trick-us-into-doing-things-we-loathe).

Arrington, Michael. 2009. "Scamville: The Social Gaming Ecosystem Of Hell." TechCrunch. Retrieved November 1, 2009 (http://www.techcrunch.com/2009/10/31/scamville-the-social-gaming-ecosystemof-hell/).

Bartle, Richard A. 2004. Designing virtual worlds. Indianapolis, Ind.: New Riders Pub.

193This bibliography helps differentiate my primary from secondary data sources. It lists only primary sources. For a complete list of sources, please see the Bibliography on page 356, which contains these industry publications as well as references to my secondary data (i.e. peer-reviewed academic texts). 
Bartle, Richard A. 1996. "Hearts, Clubs, Diamonds, Spades: Players Who Suit MUDS." Retrieved (http://mud.co.uk/richard/hcds.htm).

Bateman, Chris. 2010. "Sicart \& Bateman (2): Solitary Play.” International Hobo. Retrieved February 2, 2011 (http://blog.ihobo.com/2010/07/sicart-bateman-2solitary-play.html).

Benton, Joel. 2010. "The Future of the Independent." in Generator Networking Event: Views \& Experience From Close By and Far Away. bitHeads Studio, Ottawa, Canada.

Bethke, Ray, Min Kin, Ray Muzyka, and Nicolay Nickelsen. 2010. “The Great MMO Hope." Retrieved (http://audio.sxsw.com/2010/podcasts/031510i_TheGreatMMOHope.mp3).

Birke, Chris. 2011. "Ethos Before Analytics.” Gamasutra. Retrieved October 5, 2011 (http://www.gamasutra.com/view/feature/6487/ethos_before_analytics.php? print=1).

Bishop, Todd. 2011. "How Valve Experiments with the Economics of Video Games." Geek Wire. Retrieved November 8, 2011

(http://www.geekwire.com/2011/experiments-video-game-economics-valvesgabe-newell).

Blair, Lucas. 2011. "The Cake Is Not a Lie: How to design effective achievements, Part 2." Gamasutra. Retrieved May 20, 2011

(http://www.gamasutra.com/view/feature/6375/the_cake_is_not_a_lie_how_to_.p hp?print=1).

Blizzard Entertainment. 2010. "Battle.net ${ }^{\circledR}$ and Facebook Integration Announced." Retrieved July 29, 2010 (http://us.blizzard.com/enus/company/press/pressreleases.html?100505).

Bogost, Ian. 2010. "Cow Clicker: The making of obsession." Ian Bogost - Videogame Theory, Criticism, Design. Retrieved July 22, 2010 (http://www.bogost.com/blog/cow_clicker_1.shtml).

Brathwaite, Brenda. 2011a. "Developing Self-Discipline.” Applied Game Design. Retrieved January 17, 2011 (http://bbrathwaite.wordpress.com/2011/01/16/developing-self-discipline/).

Brathwaite, Brenda. 2011b. "Social Game Developers Rant Back." Applied Game Design. Retrieved March 7, 2011 (http://bbrathwaite.wordpress.com/2011/03/03/gdc-2011-social-game-developersrant-back/). 
Brathwaite, Brenda. 2010. "Train (or How I Dumped Electricity and Learned to Love Design)." in GDC 2010, GDC Vault. www.gdcvault.com.

Brathwaite, Brenda. 2011c. "Who Directed Star Wars?” Applied Game Design. Retrieved November 16, 2011 (http://bbrathwaite.wordpress.com/2011/11/11/who-directedstar-wars/).

Brightman, James. 2011a. "Violent Games Can Hinder Development of Empathy in Children, says Study.” Industry Gamers. Retrieved April 6, 2011 (http://www.industrygamers.com/news/violent-games-can-hinder-developmentof-empathy-in-children-says-study/).

Brightman, James. 2011b. "Zynga Rallies Against Criticism of Metrics Approach." Industry Gamers. Retrieved March 31, 2011 (http://www.industrygamers.com/news/zynga-rallies-against-criticism-of-metricsapproach/).

Brown, Nathan. 2011. "Wilson: EA Sports Football World a 'gut-wrenching change'.” Edge Magazine. Retrieved January 26, 2012 (http://www.edgeonline.com/news/wilson-ea-sports-football-world-gut-wrenching-change).

Bruno, Jose R. Gonzalez. 2010. "Tyranny of the Masses: Why developers should be weary of tracking player behavior." Game Reader. Retrieved December 17, 2010 (http://gamereader.net/2010/12/07/tyranny-of-the-masses/).

Caoili, Eric. 2011. “66\% Of U.S. Online Population Are Casual Gamers.” Worlds in Motion.Biz. Retrieved February 7, 2011 (http://worldsinmotion.biz/2011/02/study_66_of_us_online_populati.php).

Caoili, Eric. 2010a. "Playdom's Siegel: Indies need to develop social games." Worlds in Motion.Biz. Retrieved November 12, 2010 (http://worldsinmotion.biz/2010/11/migs_2010_playdoms_siegel_indi.php\#more).

Caoili, Eric. 2010b. "Playfish's Valadares on Intuition Versus Metrics: 'Make your own decisions'." Worlds in Motion.Biz. Retrieved August 17, 2010 (http://worldsinmotion.biz/2010/08/gdc_europe_playfishs_valadares.php).

Caoili, Eric. 2010c. "Wizard101 And How Social Games Influence MMOs." Worlds in Motion.Biz. Retrieved August 13, 2010 (http://worldsinmotion.biz/2010/08/interview_wizard101_and_how_so.php).

Carmel, Ron. 2010. "Indies and Publishers: Fixing a system that never worked." in Indy Games Summit 2010. www.gdcvault.com.

Castle, Lou. 2011. “Zynga's Castle On Social Game Metrics.” Gamasutra. Retrieved June 22, 2011 
(http://www.gamasutra.com/view/news/35373/GDC_Europe_Speaker_Spotlight_ Zyngas_Castle_On_Social_Game_Metrics.php).

Chen, Sande. 2009. "The Social Network Game Boom.” Gamasutra: The Art \& Business of Making Games Retrieved

(http://www.gamasutra.com/view/feature/4009/the_social_network_game_boom. php?print=1).

Chiang, Oliver. 2011. “The Master of Online Mayhem.” Forbes. Retrieved February 14, 2011 (http://www.forbes.com/forbes/2011/0228/technology-gabe-newellvideogames-valve-online-mayhem.html).

Collins, Nick. 2011. "Children Who Love Video Games Have Brains Like Gamblers.” The Telegraph Retrieved November 16, 2011 (http://www.telegraph.co.uk/science/science-news/8891651/Children-who-lovevideo-games-have-brains-like-gamblers.html).

Cook, Daniel. 2011a. "GDC 2011 Game of Platform Power.” Slideshare. Retrieved March 21, 2011 (http://www.slideshare.net/danctheduck/gdc-2011-game-ofplatform-power).

Cook, Daniel. 2011b. “Player Metrics Vs. The Vocal Minority.” Gamasutra. Retrieved November 21, 2011

(http://www.gamasutra.com/view/news/38205/Opinion_Player_Metrics_Vs_The_ Vocal_Minority.php).

Cook, Daniel. 2010. "Why Videogames are so Special Contrary to Other Games.” Empyre Forum. Retrieved January 30, 2012 (http://lists.cofa.unsw.edu.au/pipermail/empyre/2010-December.txt).

Costikyan, Greg. 2009. "Mothers, Don't Let Your Children Grow Up to Be Game Developers." Play This Thing. Retrieved October 6, 2009 (http://playthisthing.com/mothers-dont-let-your-children-grow-be-gamedevelopers).

Costikyan, Greg. 2010. “Social Network Games: ARPU over design.” Gamasutra. Retrieved May 3, 2010 (http://www.gamasutra.com/blogs/GregCostikyan/20100420/4977/Social_Networ k_Games_ARPU_over_Design.php).

Crawford, Chris. 2003. Chris Crawford on game design. Indianapolis, Ind.: New Riders.

Crawford, Chris. 1984. The Art of Computer Game Design. New York City, N.Y.: McGraw-Hill Retrieved June 5, 2009 (http://www.vancouver.wsu.edu/fac/peabody/game-book/Coverpage.html). 
Crossley, Rob. 2011a. “Can Crowdsourcing Revolutionise Indie Funding?” Develop. Retrieved January 28, 2011 (http://www.develop-online.net/news/36880/Cancrowdsourcing-revolutionise-indie-funding).

Crossley, Rob. 2011b. "EA: Digital will rise above retail this year.” Develop. Retrieved January 10, 2011 (http:/www.develop-online.net/news/36709/EA-Digital-willrise-above-retail-this-year).

Crossley, Rob. 2011c. "Exhausted Insider Exposes Crunch at Kaos.” Develop. Retrieved January 17, 2011 (http://www.develop-online.net/news/36768/Exhausted-insiderexposes-crunch-at-Kaos).

Crossley, Rob. 2011d. “Game Budgets 'Have Tripled' on 3DS and PSP2.” Develop. Retrieved March 14, 2011 (http://www.develop-online.net/news/37269/Gamebudgets-have-tripled-on-3DS-and-PSP2).

Crossley, Rob. 2011e. "LA Noire studio accused of 'thankless crunch'.” Develop. Retrieved June 20, 2011 (http://www.develop-online.net/news/38072/LA-Noirestudio-accused-of-thankless-crunch-work? utm_source=feedburner\&utm_medium=feed\&utm_campaign=Feed $\% 3 \mathrm{~A}+$ developmag\%2Fifbh+\%28Develop\%29).

Crossley, Rob. 2011f. "Mobile industry to grow 15\% in 2011.” Develop. Retrieved April 8, 2011 (http://www.develop-online.net/news/37452/Analyst-Mobile-industry-togrow-15-in-2011? utm_source=feedburner\&utm_medium=feed\&utm_campaign=Feed $\% 3 \mathrm{~A}+$ developmag\%2Fifbh+\%28Develop\%29).

Crossley, Rob. 2011g. "New App Store Law 'is Killing Dev Revenues'.” Develop. Retrieved June 1, 2011 (http://www.develop-online.net/news/37815/New-AppStore-law-is-killing-dev-revenues).

Crossley, Rob. 2011h. "Rumour: Android charting game-time, not installs." Develop. Retrieved April 8, 2011 (http://www.develop-online.net/news/37456/RumourAndroid-charting-game-time-not-installs).

Crossley, Rob. 2011i. “Sony: Future game tech could read your emotions.” Develop. Retrieved August 29, 2011 (http://www.develop-online.net/news/38493/SonyFuture-game-tech-could-read-your-emotions).

Crossley, Rob. 2011j. “Valve: Five interviews.” Develop. Retrieved May 17, 2011 (http://www.develop-online.net/features/1188/Valve-Five-interviews).

Curtis, Tom. 2012. "38 Studios, Big Huge Games Lay Off Their Entire Staff." Gamasutra. Retrieved August 28, 2012 (http://gamasutra.com/view/news/171015/38_Studios_Big_Huge_Games_lay_off 
_their_entire_staff.php).

Dallman, Joshua. 2011. "Redesigning Wild Ones into Playdom's Top Game: A social game design reboot." Gamasutra. Retrieved April 29, 2011

(http://www.gamasutra.com/view/feature/6362/redesigning_wild_ones_into_.php ?print=1).

Davison, John. 2010. "Too Big and Too Hard." GamePro. Retrieved May 5, 2010 (http://www.gamepro.com/article/news/215033/too-big-and-too-hard/).

Deejay. 2011. "The False Promise of Digital Distribution." Develop. Retrieved February 18, 2011 (http://www.develop-online.net/news/37053/OPINION-The-falsepromise-of-digital-distribution).

Della Rocca, Jason. 2009. "MIGS Keynote." in Montreal International Game Summit. Montréal, Canada.

Della Rocca, Jason. 2010. “Playing For Our Future.” The Escapist Retrieved June 23, 2010 (http://www.escapistmagazine.com/articles/view/issues/issue_252/7504Playing-For-Our-Future).

Deterding, Sebastian. 2011. "A Quick Buck by Copy and Paste." Gamification Research Network. Retrieved October 5, 2011 (http://gamification-research.org/2011/09/aquick-buck-by-copy-and-paste/).

Deterding, Sebastian. 2010a. "Pawned: Gamification and its discontents." in Playful 2010. Conway Hall, London Retrieved November 4, 2010

(http://www.slideshare.net/dings/pawned-gamification-and-its-discontents).

Dibbell, Julian. 2006. Play Money: Or, how I quit my day job and made millions trading virtual loot. New York City, N.Y.: Basic Books.

Donovan, Tristan. 2010. Replay: The history of video games. East Sussex, UK: Yellow Ant.

Dyer, Mitch. 2011. "12 Game Studios That Died in 2011." IGN. Retrieved August 28, 2012 (http://ca.ign.com/articles/2011/12/01/12-game-studios-that-died-in-2011).

Eco, Umberto. 2010. "SPIEGEL Interview with Umberto Eco: 'We Like Lists Because We Don't Want to Die'." Spiegel Online. Retrieved November 30, 2010 (http://www.spiegel.de/international/zeitgeist/0,1518,659577,00.html).

Edery, David, and Ethan Mollick. 2009. Changing the Game. Upper Saddle River, New Jersey: FT Press.

Electronic Entertainment Design and Research. 2011. "Alternative Business Models with 
DLG \& DLC.” www.eedar.com. Retrieved March 23, 2011

(http://www.gamasutra.com/view/news/33648/EEDAR_55_Of_Xbox_360_Owne

rs_Purchased_A_Downloadable_Game_In_2010.php).

Elliott, Phil. 2011a. “Alan Miller: Part 1 Interview.” gamesindustry.biz. Retrieved

February 18, 2011 (http://www.gamesindustry.biz/articles/2011-02-16-alan-millerinterview).

Elliott, Phil. 2011b. "Justin Johnson Interview." gamesindustry.biz. Retrieved March 2, 2011 (http://www.gamesindustry.biz/articles/2011-02-23-justin-johnsoninterview).

Elliott, Phil. 2010. "Metrics the Key to Successful Social Game Design." gamesindustry.biz. Retrieved July 28, 2010

(http://www.gamesindustry.biz/articles/metrics-the-key-to-successful-socialgame-design-zynga).

Elliott, Phil. 2011c. “XBLA Publishing 'Massively' Harder Now - Brown.” gamesindustry.biz. Retrieved July 28, 2011

(http://www.gamesindustry.biz/articles/xbla-publishing-massively-harder-nowbrown).

Entertainment Software Association. 2010. Essential Facts about the Computer and Video Game Industry: 2010 sales, demographic and usage data. Retrieved August 4, 2011 (http://www.theesa.com/).

Evans, Jon. 2010. "Dear Foursquare, Gowalla: Please let's stop pretending this is fun.” TechCrunch. Retrieved November 15, 2010 (http://techcrunch.com/2010/11/13/foursquare-gowalla-stop-pretending-fun/).

Fahey, Mike. 2011. "Dragon Age II Dev Rates His Own Game On Metacritic, EA Bets Obama Voted For Himself Too.” Kotaku. Retrieved March 15, 2011 (http://kotaku.com/\#!5782097/dragon-age-ii-dev-rates-his-own-game-onmetacritic-ea-bets-obama-voted-for-himself-too).

Fahey, Rob. 2011a. "Beachside Burnout." gamesindustry.biz. Retrieved July 8, 2011 (http:/www.gamesindustry.biz/articles/2011-07-08-beachside-burnout-editorial).

Fahey, Rob. 2010. “Game of Life.” gamesindustry.biz. Retrieved August 6, 2010 (http://www.gamesindustry.biz/articles/2010-08-06-game-of-life-editorial? page $=1$ ).

Fahey, Rob. 2011b. "Rock and a Hard Place." gamesindustry.biz. Retrieved May 6, 2011 (http://www.gamesindustry.biz/articles/2011-05-06-rock-and-a-hard-placeeditorial). 
Fahey, Rob. 2012. "The End of the Console Era?" Gamesindustry International. Retrieved June 22, 2012 (http:/www.gamesindustry.biz/articles/2012-06-22-theend-of-the-console-era).

Fenn, Jackie, and Mark Raskino. 2008. Mastering the Hype Cycle: How to choose the right innovation at the right time. Boston MA: Harvard Business School Press.

Fullerton, Tracy. 2008. Game Design Workshop: A playcentric approach to creating innovative games. 2nd ed. Burlington MA: Morgan Kaufmann Publishers.

Funcom. 2012. "Funcom Reduces Operational Costs Following 'The Secret World' Launch." www.funcom.com. Retrieved August 28, 2012

(http://www.funcom.com/news/funcom_reduces_operational_costs_following_the _secret_world_launch).

Gasse, Nicholas. 2011. "Fan Funding.” gamesindustry.biz. Retrieved April 18, 2011 (http://www.gamesindustry.biz/articles/2011-04-18-fan-funding-article?page=1).

Gaudiosi, John. 2011. “Mobile Will Become 'The Console of The Future,' says Developer.” Industry Gamers. Retrieved October 24, 2011 (http://www.industrygamers.com/news/mobile-will-become-the-console-of-thefuture-says-developer/).

Gibson, Rick. 2010. “Do We Still Need Publishers in an Online World?” Develop (104):12-16.

Gibson, Rick. 2011. “The Problem with Game Analytics.” Develop. Retrieved March 10, 2011 (http://www.develop-online.net/news/37244/OPINION-The-problem-withgame-analytics).

Goldberg, Harold. 2011. All Your Base Are Belong to Us: How fifty years of videogames conquered pop culture. New York City, N.Y.: Three Rivers Press.

Gouglas, Sean et al. 2010. Computer Games and Canada's Digital Economy: The role of universities in promoting innovation. SSHRC Retrieved

(http://ra.tapor.ualberta.ca/ circa/wpcontent/uploads/2010/03/ComputerGamesAndCanadasDigitalEconomy1.pdf).

Graft, Kris. 2010. “Around The Block With Brian Fargo.” Gamasutra. Retrieved January 17,2011 (http://www.gamasutra.com/view/feature/6256/around_the_block_with_brian_far go.php).

Graft, Kris. 2011a. "How Shaba's Ex-Boss Escaped From The 'Great Middle'.” Gamasutra. Retrieved May 27, 2011 (http://www.gamasutra.com/view/news/34798/Interview_How_Shaba_ExBoss_E 
scaped_From_The_Great_Middle.php).

Graft, Kris. 2011b. "Iwata: Dev costs for high-end 3DS games may approach Wii costs." Gamasutra. Retrieved July 28, 2011

(http://www.gamasutra.com/view/news/29067/Iwata_Dev_Costs_For_HighEnd_3 DS_Games_May_Approach_Wii_Costs.php).

Graft, Kris. 2011c. "Tretton: Platforms must have 'cutting edge' tech at launch to stay relevant.” Gamasutra. Retrieved April 8, 2011 (http://www.gamasutra.com/view/news/34012/Tretton_Platforms_Must_Have_Cu tting_Edge_Tech_At_Launch_To_Stay_Relevant.php).

Handrahan, Matthew. 2011a. "Freemium: Is it evil?" gamesindustry.biz. Retrieved July 28, 2011 (http://www.gamesindustry.biz/articles/2011-07-26-free-is-it-evil-blogentry).

Handrahan, Matthew. 2011b. "Rise in XBLA Prices Bucking Digital Trend." gamesindustry.biz. Retrieved July 29, 2011

(http:/www.gamesindustry.biz/articles/2011-07-22-average-price-of-xbla-gamescreeping-up).

Hawkins, Trip. 2010. "The Movie: Fact and fiction.” Digital Chocolate. Retrieved October 13, 2010 (http://blog.digitalchocolate.com/?p=656).

Hecker, Chris. 2010. “Achievements Considered Harmful?” in GDC 2010, GDC Vault. www.gdcvault.com Retrieved May 10, 2010 (http://www.gamasutra.com/view/news/27646/GDC_Heckers_Nightmare_Scenari o_A_Future_Of_Rewarding_Players_For_Dull_Tasks.php).

Herman, Leonard. 1997. Phoenix: The fall and rise of video games. 2nd ed. Springfield, NJ: Rolenta Press.

Herz, J. C. 1997. Joystick Nation: How videogames ate our quarters, won our hearts, and rewired our minds. New York City, N.Y.: Little Brown and Company.

Holtman, Jason. 2009. "Games Entertainment in the Age of Connectivity." in Montreal International Game Summit. Montréal, Canada.

Huling, Ray. 2010. “Gamification: Turning work into play.” $h+$. Retrieved September 10, 2010 (http://www.hplusmagazine.com/articles/art-entertainment/gamificationturning-work-play).

Hyman, Paul. 2007. "For Better or Worse: A quality of life update." Game Developer Magazine (June/July):7-11.

Hyman, Paul. 2010. “Targeted Focus, Broad Audience?” Gamasutra. Retrieved June 8, 
2010

(http://www.gamasutra.com/view/feature/5829/targeted_focus_broad_audience.ph p).

International Game Developers Association. 2004. “Quality of Life White Paper.” Retrieved May 5, 2011 (http://www.igda.org/quality-life-white-paper-info).

Irwin, Mary Jan. 2008. "Cooking Up A Blockbuster Game." Forbes. Retrieved July 15, 2012 (http://www.forbes.com/2008/11/21/games-eedar-developers-tech-ebizcx_mji_1121eedar.html).

Järvinen, Aki. 2011. “Book Project on Hold.” Games for Social Networks: Notes On The Design and Business of Networked Play. Retrieved February 1, 2011 (http://games4networks.posterous.com/book-project-on-on-hold).

Järvinen, Aki. 2010. "My Working Definition of \#socialgames." Games for Social Networks: Notes On The Design and Business of Networked Play. Retrieved July 21, 2010 (http://games4networks.posterous.com/my-working-definition-ofsocialgames).

Jayson, Sharon. 2011. "Don't study the video game, study the player." USA Today. Retrieved September 18, 2011 (http://yourlife.usatoday.com/health/story/2011-0914/Dont-study-the-video-game-study-the-player/50406018/1?source=twitter).

Jenkins, Henry. 1999. Congressional Testimony on Media Violence. Washington, D.C. Retrieved (http://web.mit.edu/comm-forum/papers/jenkins_ct.html).

Johnson, Soren. 2010. "Fear and Loathing in Farmville." Designer Notes. Retrieved March 22, 2010 (http://www.designer-notes.com/?p=195).

Johnson, Soren. 2009. "The Case for Metacritic." Designer Notes. Retrieved January 26, 2012 (http://www.designer-notes.com/?p=130).

Johnson, Steven B. 2005. Everything Bad is Good for You: How today's popular culture is actually making us smarter. New York City, N.Y.: Riverhead Books.

Kelly, Tadhg. 2010. "Fight Design Bloat with Data." Gamasutra. Retrieved September 16,2010 (http://www.gamasutra.com/blogs/TadhgKelly/20100910/5803/Fight_Design_Blo at_with_Data.php).

Kent, Steven L. 2001. The Ultimate History of Video Games: From Pong to Pokemon. New York City, N.Y.: Three Rivers Press.

Kim, Amy Jo. 2000. Community Building on the Web : Secret strategies for successful online communities. Berkeley, CA: Peachpit Press. 
Kim, Amy Jo. 2010. "MetaGame Design: Reward systems that drive engagement." in Social Games Summit at GDC 2010. Retrieved (www.gdcvault.com).

Kim, Amy Jo. 2009. "Putting the Fun in Functional: Applying game mechanics to functional software.” in Google TechTalks. Retrieved (http://www.youtube.com/watch?v=ihUt-163gZI).

Kohler, Chris. 2010. "Farm Wars: How Facebook games harvest big bucks." Wired. Retrieved July 28, 2010 (http://www.wired.com/gamelife/2010/05/farmwars/all/1).

Koster, Raph. 2002. “A Philosophical Statement on Playerkilling.” Retrieved (http://www.raphkoster.com/gaming/pkphilosophy.shtml).

Koster, Raph. 2005. A Theory of Fun for Game Design. Scottsdale, AZ: Paraglyph Press.

Koster, Raph. 2010. “Gameifying Everything.” Raph Koster's Website. Retrieved September 14, 2010 (http://www.raphkoster.com/2010/02/18/gameifyingeverything/).

Lai, Albert. 2011. "7 Top Social Game Metrics for 2011: The A.R.M metrics framework." Slideshare. Retrieved March 16, 2011 (http://www.slideshare.net/kontagent/kontagent-top-7-deadly-arms-metrics-forsocial-games).

Lanier, Jaron. 2010. You Are Not a Gadget: A Manifesto. New York City, N.Y.: Random House.

Lovell, Nicholas. 2011a. "Does Zynga Intentionally Kick Players Out of its Games After Two Months?" GAMESbrief. Retrieved June 6, 2011

(http://www.gamesbrief.com/2011/06/does-zynga-intentionally-kick-players-outof-its-games-after-two-months).

Lovell, Nicholas. 2010. "Four Reasons Why VCs Won't Fund Game Companies." Gamasutra. Retrieved July 28, 2010 (http://www.gamasutra.com/blogs/NicholasLovell/20100324/4758/Four_Reasons _Why_VCs_Wont_Fund_Game_Companies.php).

Lovell, Nicholas. 2011b. "Whales, Dolphins and Minnows: The beating heart of a free-toplay game.” GAMESbrief. Retrieved November 21, 2011 (http://www.gamesbrief.com/2011/11/whales-dolphins-and-minnows-the-beatingheart-of-a-free-to-play-game).

Luscombe, Belinda. 2009. “Are You Getting Scammed by Facebook Games?” Time Retrieved May 11, 2010 (http:/www.time.com/time/business/article/0,8599,1935698,00.html). 
Lyons, Daniel. 2009. "Is Facebook a Paradise for Scammers?” Newsweek Retrieved May 11, 2010 (http://blog.newsweek.com/blogs/techtonicshifts/archive/2009/11/06/isfacebook-a-paradise-for-scammers.aspx).

Mangalindan, J. P. 2010. "Play to Win: The game-based economy." Fortune. Retrieved September 9, 2010 (http://tech.fortune.cnn.com/2010/09/03/the-game-basedeconomy/).

Martin, Matt. 2011a. “OnLive's Steve Perlman Interview.” gamesindustry.biz. Retrieved June 27, 2011 (http://www.gamesindustry.biz/articles/2011-06-27-onlives-steveperlman-interview).

Martin, Matt. 2011b. “The Hard Cell Interview.” gamesindustry.biz. Retrieved February 28, 2011 (http://www.gamesindustry.biz/articles/2011-02-22-the-hard-cellinterview).

McDevitt, Darby. 2010. "The Deaths Of Game Narrative.” Gamasutra. Retrieved August 5,2010

(http://www.gamasutra.com/view/feature/5952/the_deaths_of_game_narrative.ph p).

McGonigal, Jane. 2011. Reality Is Broken: Why games make us better and how they can change the world. London, UK: Penguin Press.

Meer, Alec. 2011a. “Deathmapping: Just Cause 2's 11m casualties.” Rock Paper Shotgun. Retrieved May 27, 2011 (http:/www.rockpapershotgun.com/2011/05/27/justcause-2-deaths-video/).

Meer, Alec. 2011b. “Indie Godfather Cliff Harris Interview.” gamesindustry.biz. Retrieved January 18, 2011 (http:/www.gamesindustry.biz/articles/2011-01-18indie-godfather-cliff-harris-interview).

Meer, Alec. 2011c. "LOTRO revenues up 3x since free to play switch.” Retrieved January 7, 2011 (http://www.gamesindustry.biz/articles/2011-01-07-lotrorevenues-up-3x-since-free-to-play-switch).

Meer, Alec. 2011d. “Mode 7's Paul Taylor Interview.” gamesindustry.biz. Retrieved July 12, 2011 (http://www.gamesindustry.biz/articles/2011-07-12-mode-7s-paul-taylorinterview).

Meer, Alec. 2010a. "PopCap: This is 'The beginning of the end' of Facebook's 'Golden Era'." gamesindustry.biz. Retrieved October 13, 2010 (http://www.gamesindustry.biz/articles/2010-09-06-popcap-this-is-the-beginningof-the-end-of-facebooks-golden-era).

Meer, Alec. 2011e. "Puppy Dog Tales Interview." gamesindustry.biz. Retrieved April 4, 
2011 (http://www.gamesindustry.biz/articles/2011-04-04-puppy-dogs-talesinterview).

Meer, Alec. 2010b. “Zynga Valued at \$5.51bn.” gamesindustry.biz. Retrieved October 26, 2010 (http://www.gamesindustry.biz/articles/2010-10-26-zynga-valued-at-USD5$51 \mathrm{bn})$.

Merel, Tim. 2011. "Money Games: Online, mobile, China and more." gamesindustry.biz. Retrieved March 14, 2011 (http://www.gamesindustry.biz/articles/2011-03-10money-games-online-mobile-china-and-more-article?page=1).

Morrison, Chris. 2010a. "Inside a Small Developer's Success on Facebook: 5th Planet's Dawn of the Dragons." Inside Social Games. Retrieved July 21, 2010 (http://www.insidesocialgames.com/2010/07/21/dawn-of-the-dragons-facebookgame-5th-plane).

Morrison, Chris. 2010b. "The Future Looks Bright for Small Social Game Developers on Facebook.” Inside Social Games. Retrieved September 28, 2010

(http://www.insidesocialgames.com/2010/09/28/the-future-looks-bright-for-smalldevelopers-on-facebook).

Newman, Ryan, and Brandon Sheffield. 2011. "Tenth Annual Salary Survey." Game Developer Magazine (April):7 -13.

Ninja Metrics. 2011. "Katana Engine and Game Analytics Software.” Retrieved January 19, 2012 (http://ninjametrics.com/).

Nutt, Christian. 2011a. “A Philosophy That Extends Eastward: Social games Zyngastyle.” Gamasutra. Retrieved February 7, 2011 (http://www.gamasutra.com/view/feature/6280/a_philosophy_that_extends_.php).

Nutt, Christian. 2011b. "EVE Online: The next steps." Gamasutra. Retrieved March 14, 2011

(http://www.gamasutra.com/view/feature/6302/eve_online_the_next_steps.php? print $=1$ ).

Nutt, Christian. 2011c. "Perfecting The Free-To-Play Battlefield Heroes." Gamasutra. Retrieved March 7, 2011 (http://www.gamasutra.com/view/news/33371/GDC_2011_Perfecting_The_FreeT oPlay_Battlefield_Heroes.php).

Nutt, Christian. 2011d. "Schafer Admits Fantasy Of Flatulence On Youth." Gamasutra. Retrieved February 14, 2011 (http://www.gamasutra.com/view/feature/6284/schafer_admits_fantasy_of_.php? print=1). 
Nutt, Christian. 2010. "Social the CrowdStar Way." Gamasutra. Retrieved November 15, 2010

(http://www.gamasutra.com/view/feature/6204/social_the_crowdstar_way.php? print=1).

Nutt, Christian. 2011e. "The Secrets Of Wooga's Social Game Success." Gamasutra.

Retrieved October 5, 2011

(http://www.gamasutra.com/view/feature/6488/interview_the_secrets_of_woogas _.php?print=1).

Olivarez-Giles, Nathan. 2011. "Facebook F8: Redesigning and hitting 800 million users." L.A. Times, online Retrieved January 9, 2012 (http://latimesblogs.latimes.com/technology/2011/09/facebook-f8-mediafeatures.html).

Orland, Kyle. 2011a. "Facebook Changes Method For Measuring Active App Users." Gamasutra. Retrieved January 24, 2012

(http://www.gamasutra.com/view/news/37921/Facebook_Changes_Method_For_ Measuring_Active_App_Users.php).

Orland, Kyle. 2011b. "Minecraft Draws Over \$33 Million In Revenue From 1.8M Paying Customers.” Gamasutra. Retrieved April 8, 2011

(http://www.gamasutra.com/view/news/33961/Minecraft_Draws_Over_33_Millio n_In_Revenue_From_18M_Paying_Customers.php).

Orland, Kyle. 2011c. "New Research Suggests Potential Causes For 'Problem Gaming'.” Gamasutra. Retrieved February 7, 2011

(http://www.gamasutra.com/view/news/32853/New_Research_Suggests_Potential _Causes_For_Problem_Gaming.php?

utm_source $=$ feedburner\&utm_medium $=$ feed\&utm_campaign $=$ Feed

$\% 3 \mathrm{~A}+$ GamasutraNews+\%28Gamasutra+News\%29).

Orland, Kyle. 2011d. “Zynga Stock To Be Listed On NASDAQ.” Gamasutra. Retrieved October 17, 2011

(http://www.gamasutra.com/view/news/37898/Zynga_Stock_To_Be_Listed_On_ NASDAQ.php).

Parkin, Simon. 2010a. "BioWare's Zeschuk Calls AAA Development 'A Poor Goal' Today." Gamasutra. Retrieved July 14, 2010

(http://www.gamasutra.com/view/news/29421/Develop_BioWares_Zeschuk_Call S_AAA_Development_A_Poor_Goal_Today.php).

Parkin, Simon. 2010b. “Double Fine's Schafer On 'Amnesia Fortnights' And The Pitfalls Of AAA." Gamasutra. Retrieved July 15, 2010 (http://www.gamasutra.com/view/news/29450/Develop_Double_Fines_Schafer_ 
On_Amnesia_Fortnights_And_The_Pitfalls_Of_AAA.php).

Pearson, Dan. 2011. "You Had Me At Hello Interview." gamesindustry.biz. Retrieved July 27, 2011 (http://www.gamesindustry.biz/articles/2011-07-25-hello-games-seanmurray-interview?page=2).

Phillips, Bruce. 2009. "Staying Power: Rethinking feedback to keep players in the game." Gamasutra. Retrieved November 28, 2011 (http://www.gamasutra.com/view/feature/4171/staying_power_rethinking_feedba ck_php?print=1).

Plunkett, Luke. 2012. “Every Game Studio That's Closed Down Since 2006.” Kotaku. Retrieved August 28, 2012 (http://kotaku.com/5876693/every-game-studio-thatsclosed-down-since-2006).

Plunkett, Luke. 2011. "Nintendo 3DS Gets Sudden, Massive Price Drop.” Kotaku. Retrieved July 28, 2011 (http://kotaku.com/5825495/nintendo-3ds-gets-suddenmassive-price-drop).

Poole, Steven. 2000. Trigger Happy: Videogames and the entertainment revolution. New York City, N.Y.: Arcade Publishing.

Poppendieck, Mary, and Tom Poppendieck. 2009. Leading Lean Software Development: Results are not the point. 1st ed. Upper Saddle River, NJ: Addison-Wesley Professional.

Priebatsch, Seth. 2010. "The Game Layer on Top of the World." Retrieved September 9, 2010 (http://tedxboston.org/videos/176-seth-priebatsch-the-game-layer-on-top-ofthe-world).

Pruett, Chris. 2010. "Hot Failure: Tuning gameplay with simple player metrics." Gamasutra. Retrieved December 16, 2010

(http://www.gamasutra.com/view/feature/6155/hot_failure_tuning_gameplay_wit h_.php?print=1).

Radd, David. 2011a. "NPD: Number of children gamers increasing." Industry Gamers. Retrieved October 12, 2011 (http://www.industrygamers.com/news/npd-numberof-children-gamers-increasing/).

Radd, David. 2011b. "PC Gaming: Death and rebirth.” Industry Gamers. Retrieved November 21, 2011 (http://www.industrygamers.com/news/pc-gaming-death-andrebirth/).

Reader, Pauline. 2010. "Monetizing Social Games." Proceedings of GDC 2010 in GDC Vault. Retrieved January 26, 2012 (http://www.gdcvault.com/play/1013731/Monetizing-Social). 
Reeves, Byron, and J. Leighton Read. 2009. Total Engagement: Using games and virtual worlds to change the way people work and businesses compete. Boston MA:

Harvard Business Press.

Remo, Chris. 2010a. "Controversy Erupts Over Rockstar San Diego Employee Allegations." Gamasutra. Retrieved December 16, 2010 (http://www.gamasutra.com/view/news/26803/Controversy_Erupts_Over_Rockst ar_San_Diego_Employee_Allegations.php).

Remo, Chris. 2010b. "Developers Claim 13 Weeks Of Crunch Per Year.” Gamasutra. Retrieved June 2, 2010 (http://www.gamasutra.com/view/news/28669/Study_Developers_Claim_13_Wee ks_Of_Crunch_Per_Year.php).

Ries, Eric. 2011. The Lean Startup: How today's entrepreneurs use continuous innovation to create radically successful businesses. New York City, N.Y.: Crown Business.

Robertson, Margaret. 2010. "Can't Play, Won't Play.” Hide \& Seek: Inventing new kinds of play. Retrieved November 4, 2010 (http://www.hideandseek.net/cant-playwont-play/).

Robinson, Erin. 2011. "The Top 10 Weird Children Of Video Games and Neuroscience." Gamasutra. Retrieved August 29, 2011 (http://www.gamasutra.com/view/feature/6466/the_top_10_weird_children_of_vi deo_.php?print=1).

Rose, Mike. 2011a. “Nintendo 'Not Interested’ In Free-To-Play Model.” Gamasutra. Retrieved June 16, 2011 (http://www.gamasutra.com/view/news/35276/Nintendo_Not_Interested_In_Free ToPlay_Model.php).

Rose, Mike. 2011b. "Sony Reveals \$20M Investment Plan For PSN-Exclusive Titles." Gamasutra. Retrieved July 14, 2011 (http://www.gamasutra.com/view/news/35840/Sony_Reveals_20M_Investment_P lan_For_PSNExclusive_Titles.php).

Rose, Mike. 2011c. "Steam Revenue For Zeboyd Games RPGs Outpaces Lifetime Xbox Mark In 6 Days.” Gamasutra. Retrieved July 19, 2011 (http://www.gamasutra.com/view/news/35957/Steam_Revenue_For_Zeboyd_Ga mes_RPGs_Outpaces_Lifetime_Xbox_Mark_In_6_Days.php).

Rusli, Evelyn M. 2011a. “As Net Stocks Sizzle, Zynga Aims High for Its I.P.O.” The New York Times, online Retrieved January 10, 2012 (http://dealbook.nytimes.com/2011/07/01/zynga-files-for-1-billion-i-p-o/). 
Rusli, Evelyn M. 2011b. "Zynga Nears Deal Valuing It at Close to \$10 Billion." New York Times. Retrieved February 18, 2011

(http://dealbook.nytimes.com/2011/02/17/zynga-nears-deal-valuing-it-at-close-to10-billion/).

Schell, Jesse. 2010a. "Design Outside the Box: Beyond Facebook." in DICE 2010. Retrieved (http://www.g4tv.com/videos/44277/dice-2010-design-outside-the-boxpresentation/).

Schell, Jesse. 2010b. “TED TV: When games invade real life.” Retrieved November 16, 2010

(http://www.ted.com/talks/jesse_schell_when_games_invade_real_life.html).

Schell, Jesse. 2008. The Art of Game Design: A book of lenses. Burlington MA: Morgan Kaufmann.

Schell, Jesse. 2010c. "Visions of the Gamepocalypse." FORA TV. Retrieved November 16,2010 (http://fora.tv/2010/07/27/Jesse_Schell_Visions_of_the_Gamepocalypse).

Schwab, Stephanie. 2010. "Gamification: What is it and why do you need to know?" Stephanie Schwab: Socialologist. Retrieved September 22, 2010 (http://www.stephanieschwab.com/2010/07/07/gamification/).

Sheff, David. 1993. Game Over: How Nintendo zapped an American industry, captured your dollars, and enslaved your children. New York City, N.Y.: Random House.

Sheffield, Brandon. 2010. “Super Rewards' Bailey: 'Quit Your Job And Make Facebook Games'.” Gamasutra. Retrieved May 11, 2010

(http://www.gamasutra.com/view/news/28456/GDC_Canada_Super_Rewards_Ba iley_Quit_Your_Job_And_Make_Facebook_Games.php).

Shipman, Matt. 2011. "What Gamers Want: Researchers develop tool to predict player behavior." North Carolina State University Newsroom. Retrieved June 16, 2011 (http://news.ncsu.edu/releases/wmsrobertspredict/).

Siegel, Scott Jon. 2010. "David v.s. Goliathville: Sage advice for indie social game designers.” in Montréal International Games Summit. Montréal, Canada.

Siegel, Scott Jon. 2009. "Long-Tail Game Design: Building successful games for social networks.” in Montréal International Games Summit. Montréal, Canada.

Sinclair, Brenden. 2010. "Spot On: Social anxiety.” Gamespot. Retrieved November 25, 2010 (http://www.gamespot.com/news/6284524.html).

Sliwinski, Alexander. 2012. “‘38 Spouse’ Explains Decision to Speak Now, Never Heard 
of 'EA Spouse'." Joystiq. Retrieved August 29, 2012

(http://www.joystiq.com/2012/06/14/38-studios-spouse-38-spouse/).

Strauss, Ben. 2011. "Violent Games Show Link in Altering Male Brains." Industry Gamers. Retrieved November 29, 2011

(http://www.industrygamers.com/news/violent-games-show-link-in-alteringmens-brains/).

Stuart, Keith. 2011. "The Metrics are the Message: How analytics is shaping social games." Guardian UK. Retrieved July 19, 2011 (http://www.guardian.co.uk/technology/gamesblog/2011/jul/14/social-gamingmetrics).

Su, Mike. 2010. "Social Gaming: Where we've been, and where we're headed." TechCrunch. Retrieved November 15, 2010 (http://techcrunch.com/2010/11/13/social-gaming).

Surowiecki, James. 2004. Wisdom of Crowds. New York City, N.Y.: Random House.

Su, Susan. 2010. “Who’s Using Facebook’s Top Apps? Demographic data indicate diverse audiences." Inside Facebook. Retrieved June 21, 2010 (http://www.insidefacebook.com/2010/06/01/whos-using-facebooks-top-appsdemographic-data-indicate-diverse-audiences/).

Takahashi, Dean. 2006. The Xbox 360 Uncloaked: The real story behind microsoft's nextgeneration video game console. Raleigh, N.C.: SpiderWorks LLC.

Takahashi, Dean. 2010. "Ubisoft to Launch Social Game Portal and First Facebook Game." Venture Beat. Retrieved July 29, 2010 (http://venturebeat.com/2009/07/17/ubisoft-to-launch-social-game-portal-andfirst-facebook-game/).

Terdiman, Dan. 2010. "Why Zynga Ticks off the Games Industry." CNET News. Retrieved July 22, 2010 (http://news.cnet.com/8301-13772_3-20002221-52.html).

Thomsen, Michael. 2011. "We're Going to Need Another Category: Going hardcore on Facebook." Gamasutra. Retrieved October 12, 2011 (http://www.gamasutra.com/view/feature/6507/were_going_to_need_another_.ph p?print=1).

Totilo, Stephen. 2008. "Low Metacritic Scores Cause Game Publishers To Withhold Developer Royalties.” MTV Geek. Retrieved January 26, 2012 (http://multiplayerblog.mtv.com/2008/05/29/low-metacritic-costs-developers/).

Valadares, Jeferson. 2011. "Consumers Spend Average of $\$ 14$ per Transaction in iOS and Android Freemium Games.” Flurry.com. Retrieved July 27, 2011 
(http://blog.flurry.com/bid/67748/Consumers-Spend-Average-of-14-perTransaction-in-iOS-and-Android-Freemium-Games).

Vanden Bossche, Andrew. 2011. "Trapdoor Studios On Warp And Small-Scale Development." Gamasutra. Retrieved May 4, 2011 (http://www.gamasutra.com/view/news/33970/Interview_Trapdoor_Studios_On_ Warp_And_SmallScale_Development.php).

Webster, Andrew. 2011a. "Smartphone and Tablet Rundown: What developers need To know." Gamasutra. Retrieved July 27, 2011 (http://www.gamasutra.com/view/feature/6440/smartphone_and_tablet_rundown_ .php?print=1).

Webster, Andrew. 2011b. "The Crowdfunding Revolution: Perspectives." Gamasutra. Retrieved April 22, 2011 (http://www.gamasutra.com/view/feature/6353/the_crowdfunding_revolution_.ph p).

Webster, Andrew. 2011c. "Xbox Live Indie Games: No way to make a living.” Ars Technica. Retrieved July 6, 2011 (http://arstechnica.com/gaming/news/2011/07/xblig-feature.ars).

Wikipedia. 2010a. "Gamification.” Wikipedia. Retrieved September 22, 2010 (http://en.wikipedia.org/wiki/Gamification).

Wikipedia. 2010b. “Gamification.” Retrieved November 16, 2010 (http://en.wikipedia.org/wiki/Gamification).

Wikipedia. 2012. “Gamification.” Wikipedia. Retrieved March 9, 2012 (http://en.wikipedia.org/wiki/Gamification).

Wikipedia. 2011. "World of Warcraft.” Wikipedia. Retrieved February 8, 2011 (http://en.wikipedia.org/wiki/World_of_Warcraft).

Williams, M.H. 2011. “Gameloft Accused Of 'Dangerous' Work Conditions.” Industry Gamers. Retrieved July 19, 2011 (http://www.industrygamers.com/news/gameloft-accused-of-dangerous-workconditions/).

Windels, Joel. 2011. "Scary Game Findings: A study of horror games and their players." Gamasutra. Retrieved September 7, 2011 (http://www.gamasutra.com/view/feature/6480/scary_game_findings_a_study_of_ .php?print=1).

Wolf, Gary. 2010. “The Data-Driven Life.” New York Times. Retrieved September 14, 2010 (http://www.nytimes.com/2010/05/02/magazine/02self-measurement-t.html? 


$$
\text { _r=1\&pagewanted=all). }
$$

Young, Neil. 2010. "Things to Unlearn Moving from Traditional Development to the New Digital World.” in GDC 2010, GDC Vault. Retrieved May 11, 2010 (http://www.gdcvault.com/free/category/280/conference/).

Zichermann, Gabe, and Christopher Cunningham. 2011. Gamification by Design:

Implementing game mechanics in web and mobile apps. Sebastopol, CA: O'Reilly Media, Inc.

Zichermann, Gabe, and Joselin Linder. 2010. Game-Based Marketing: Inspire customer loyalty through rewards, challenges, and contests. Hoboken N.J.: Wiley.

Zoeller, Georg. 2010. "GDC 2010: Development telemetry in video games projects." Georg@GDC. Retrieved March 22, 2011 (http://gdc.gulbsoft.org/talk). 


\section{Ludology ${ }^{194}$}

- $\quad$ Barr, Pippin. 2011. The Artist is Present. Pippin Bar (browser based).

- $\quad$ NC Soft. 2008. Aion. NC Soft (Windows).

- $\quad$ Rovio Entertainment. 2009. Angry Birds. Chillingo (iOS and PSP).

- $\quad$ Easy Studios and EA Digital Illusions CE. 2009. Battlefield Heroes. Electronic Arts and Aeria Games (Windows).

- $\quad$ PopCap Games. 2001. Bejeweled. PopCap Games (numerous platforms).

- Irrational Games. 2007. Bioshock. 2K Games (Playstation 3, Xbox 360, Windows).

- Gaijin Games. 2009. Bit.Trip Beat. Aksys Games (Wii).

- Lionhead Studios. 2001. Black \& White. Electronic Arts and Feral Interactive (Windows and Mac).

- Gearbox Software. 2009. Borderlands. 2K Games (Playstation 3, Xbox 360, Windows, OnLive).

- Blow, Jonathan. 2008/2009. Braid. Microsoft Game Studios and Number None, Inc. (XBLA and Windows).

- $\quad$ Double Fine. 2009. Brütal Legend. Electronic Arts (Playstation 3, Xbox 360).

- Infinity Ward. 2009. Call of Duty: Modern Warfare 2. Activision (Windows, Playstation 3, and Xbox 360).

- The Behemoth. 2008. Castle Crashers. Microsoft Game Studies (Xbox 360).

- $\quad$ Zynga. 2010. CityVille. Zynga (Facebook and Google+).

- MicroProse and Firaxis Games. (franchise 1991-). Civilization. MicroProse, Infogrames Entertainment SA, and 2K (numerous platforms).

- $\quad$ MicroProse. 1996. Civilization II. MicroProse (Windows, Mac, and Playstation).

- $\quad$ Firaxis Games. Sid Meier's Civilization V. 2K Games (Windows, Mac OS X).

- Westwood Studios. ${ }^{195}$ (franchise 1995 - ). Command \& Conquer. Electronic Arts (numerous platforms).

- Valve Corporation. Counter-Strike. Valve Corporation and Microsoft Game Studios (Windows and Xbox).

- $\quad$ Crytek Frankfurt. 2007. Crysis. Electronic Arts (Windows).

- ZeptoLab. 2010. Cut the Rope. Chillingo (numerous platforms).

- Visceral Games. 2011. Dead Space 2. Electronic Arts (Playstation 3, Xbox 360, Windows).

- GameLab. 2003. Diner Dash. PlayFirst (numerous platforms).

- Limbic Entertainment. 2011. Dungeon Empires. Gamigo (browser based).

- $\quad$ Playfish. 2010. EA Sports FIFA Superstars. Electronic Arts (Facebook).

- $\quad$ Bethesda Game Studios. 2011. The Elder Scrolls V: Skyrim. Bethesda Softworks (Windows, Playstation 3, and Xbox 360).

194Many games are ported to new platforms by different developers and published by different publishers.

This ludography only credits the original developers. 195EA took over the franchise's development in 2003. 
- CCP Games. 2003. Eve Online. Atari, Valve Corporation and Mobius Games (Windows, Mac OS X and Linux)

- $\quad$ Sony Online Entertainment. (franchise 1999- ). EverQuest. Sony Online Entertainment (Windows and Mac OS X).

- $\quad$ Evony, LLC. 2009. Evony. Evony (Adobe Flash).

- $\quad$ Slashkey. 2009. Farm Town. Slashkey (Facebook and Myspace).

- $\quad$ Zynga. 2009. Farmville. Zynga (Facebook, iOS, and HTML5).

- Titan Studios. 2009. Fat Princess. Sony Computer Entertainment (PlayStation Network).

- $\quad$ Square Enix. (franchise 1987-). Final Fantasy. Square Enix (numerous platforms).

- Zynga. 2010. FrontierVille (now The Pioneer Trail). Zynga (Facebook).

- Valve Corporation. 2004. Half-Life 2. Valve Corporation and Sierra Entertainment (Windows, Mac OS X, Xbox and Xbox 360).

- $\quad$ Bungie. 2007. Halo: 3. Microsoft Game Studios (Xbox 360).

- Bungie. 2010. Halo: Reach. Microsoft Game Studios (Xbox 360).

- $\quad$ Avalanche Studios. 2010. Just Cause 2. Eidos Interactive (Windows, Playstation 3, Xbox 360).

- 38 Studios, Big Huge Games. 2012. Kingdoms of Amalur: Reckoning. Electronic Arts (Windows, Playstation, Xbox 360).

- Turtle Rock Studios. 2008. Left 4 Dead. Valve Corporation (Windows, Xbox 360, Mac OS X).

- $\quad$ Media Molecule. 2008. Little Big Planet. Sony Computer Entertainment Europe (Playstation 3).

- Turbine, Inc. 2007. The Lord of The Rings Online. Turbine, Inc and Midway Games (Windows).

- $\quad$ Playfish. 2010. Madden NFL Superstars. Electronic Arts (Facebook).

- $\quad$ Zynga. 2008. Mafia Wars. Zynga (numerous platforms).

- $\quad$ Hudson Soft. 1998. Mario Party. Nintendo (Nintendo 64).

- BioWare. 2007. Mass Effect. Microsoft Game Studios and Electronic Arts (Xbox 360 and Windows).

- $\quad$ Mojang. 2009 (alpha). Minecraft. Mojang (Java, Android, iOS, Xbox 360).

- $\quad$ Donner, Robert. 1990. Minesweeper. Microsoft (Windows).

- $\quad$ Maestri, David. 2008. Mob Wars. David Maestri (Facebook).

- $\quad$ Double Fine. 2011. Sesame Street: Once Upon a Monster. Warner Bros. Interactive Entertainment (Xbox 360).

- $\quad$ Area/Code. 2007. Parking Wars. A\&E Television (Facebook).

- Pyramid/Japan Studio. 2007. Patapon. Sony Computer Entertainment (PlayStation Portable).

- $\quad$ PopCap Games. 2009. Plants vs. Zombies. PopCap Games and Electronic Arts (numerous platforms).

- $\quad$ PopCap Games. 2007. Peggle. PopCap Games (numerous platforms). 
- $\quad$ PlayFish. 2008. Pet Society. Playfish (Facebook, Myspace, Bebo, iOS).

- Valve Corporation. 2011. Portal 2. Valve Corporation (Windows, Mac OS X, Playstation 3 and Xbox 360).

- $\quad$ Three Rings Design. 2003. Puzzle Pirates. Three Rings Design and Ubisoft. (Java).

- $\quad$ Capcom. 2009. Resident Evil 5. Capcom (Playstation 3, Xbox 360, Windows).

- Trion Worlds. 2011. Rift. Trion Worlds (Windows).

- HandCircus. 2008. Rolando. Ngmoco (iOS).

- $\quad 5$ th Cell. 2009. Scribblenauts. WB Games and Konami (Nintendo DS).

- $\quad$ Linden Research, Inc. 2003. Second Life. Linden Research, Inc. (Windows, Mac OS $\mathrm{X}$ and Linux).

- Maxis. (franchise 2000- ). The Sims. Electronic Arts (numerous platforms).

- Taito Corporation. 1978. Space Invaders. Taito and Midway (Aracade).

- $\quad$ Russell, Steve et al. 1962. Spacewar! (DEC PDP-1).

- $\quad$ Blizzard Entertainment. 2010. Starcraft II: Wings of Liberty. Blizzard Entertainment (Windows and Mac OS X).

- $\quad$ Maxis. 2008. Spore. Electronic Arts (Windows and Mac OS X).

- $\quad$ Nintendo. (franchise 1985-). Super Mario Bros. Nintendo (numerous platforms).

- Nintendo EAD Tokyo. 2007. Super Mario Galaxy. Nintendo (Wii).

- $\quad$ Team Meat. 2010. Super Meat Boy. Steam, Direct2Drive and Xbox Live (Windows, Mac OS X, Linux and XBLA).

- $\quad$ Cherry, Wes. 1990. Solitare. Microsoft (Windows).

- Valve Corporation. 2007. Team Fortress 2. Valve Corporation (Windows, Mac OS $\mathrm{X}$, Playstation 3, and Xbox 360).

- Funcom. 2012. The Secret World. (Windows).

- $\quad$ Zynga. 2010. Treasure Isle. Zynga (Facebook).

- Origin Systems and Electronic Arts. (franchise 1997-). Ultima Online. Electronic Arts (Windows and Linux).

- Zynga with Friends (formerly Newtoy, Inc.). 2010. We Rule. Ngmoco (Android, iOS).

- $\quad$ Playfish. 2007. Who Has the Biggest Brain? Playfish (Facebook, iOS, Android).

- $\quad$ Playdom. 2010. Wild Ones. Playdom (Playdom, Facebook, Myspace, and Google+).

- $\quad$ Nintendo EAD. 2006. WiiPlay. Nintendo (Wii).

- CD Projekt RED. 2007. The Witcher. Atari, Inc. (Windows, Mac OS X, OnLive).

- KingIsle Entertainment. 2008. Wizard101. KingsIsle Entertainment and Gameforge (Windows).

- $\quad$ Zynga with Friends (formerly Newtoy, Inc.). 2009. Words With Friends. Zynga (Facebook, iOS, Android, Kindle, Nook).

- 2D Boy. 2008. World of Goo. 2D Boy and Nintendo. (Windows, Mac OS X, Linux, iOS, Android, and WiiWare).

- Blizzard Entertainment. 2004. World of Warcraft. Blizzard Entertainment 
(Windows and Mac OS X).

- $\quad$ Playfish. 2011. World Series Superstars. Electronic Arts (Facebook). 


\section{Works Cited}

Aarseth, Espen J. 1997. Cybertext: Perspectives on ergodic literature. Baltimore MD: Johns Hopkins University Press.

Acton, Mike. 2011. “Game Dev Doesn't Have to Suck.” Develop. Retrieved January 19, 2011 (http://www.develop-online.net/news/36785/OPINION-Game-developmentdoesnt-have-to-suck).

Adams, Ernest. 2010. “The Designer's Notebook: Selling Hate and Humiliation.” Gamasutra. Retrieved May 10, 2010 (http://www.gamasutra.com/view/feature/4319/the_designers_notebook_selling_. php?print=1).

Afuah, Allan, and Rosa Grimaldi. 2005. “Architectural Innovation and the Attacker's Advantage from Complementary Assets: The case of the video game console industry.” SSRN eLibrary. Retrieved April 5, 2011

(http://ssrn.com/abstract=931532).

Akrich, Madeline, and Bruno Latour. 1992. "A Summary of a Convenient Vocabulary for the Semiotics of Human and Nonhuman Assemblies." Pp. 259 - 264 in Shaping Technology/Building Society: Studies in sociotechnical change, edited by Wiebe Bijker and John Law. Cambridge MA: The MIT Press.

Albrechtslund, Anders, and Lynsey Dubbeld. 2005. "The Plays and Arts of Surveillance: Studying surveillance as entertainment." Surveillance \& Society 3(2/3):216 - 221.

Alexander, Leigh. 2011. "Balancing Metrics And Creativity Key To Item Sales In The Sims Social.” Gamasutra. Retrieved October 17, 2011

(http://www.gamasutra.com/view/news/37799/gdc_online_balancing_metrics_and -.php).

Alexander, Leigh. 2010a. “Game Violence, Real Aggression Don't Correlate In Aussie R18 Debate.” Gamasutra. Retrieved December 1, 2010

(http:/www.gamasutra.com/view/news/31797/Game_Violence_Real_Aggression _Dont_Correlate_In_Aussie_R18_Debate.php?

utm_source $=$ feedburner\&utm_medium $=$ feed \&utm_campaign=Feed

$\% 3 \mathrm{~A}+$ GamasutraNews+\%28Gamasutra+News\%29).

Alexander, Leigh. 2010b. "In An Era Of 'Anguish', Game Design Searches For Its Soul.” Gamasutra. Retrieved October 13, 2010

(http://www.gamasutra.com/view/news/30587/Analysis_In_An_Era_Of_Anguish _Game_Design_Searches_For_Its_Soul_.php).

Alexander, Leigh. 2010c. "Reynolds Pioneers For Zynga With New FrontierVille.” Gamasutra. Retrieved June 10, 2010 (http://www.gamasutra.com/view/news/28872/Interview_Reynolds_Pioneers_For 
_Zynga_With_New_FrontierVille.php).

Alexander, Leigh. 2010d. "Video Games Keep Tricking Us Into Doing Things We Loathe.” Kotaku. Retrieved November 4, 2010 (http://kotaku.com/5678356/videogames-can-trick-us-into-doing-things-we-loathe).

Aoyama, Yuko, and Hiro Izushi. 2008. "User-led Innovation and the Video Game Industry." Pp. 1-22 in IRP Conference Proceedings. London, UK: DIME Working Papers.

Arrington, Michael. 2009. "Scamville: The Social Gaming Ecosystem Of Hell." TechCrunch. Retrieved November 1, 2009 (http://www.techcrunch.com/2009/10/31/scamville-the-social-gaming-ecosystemof-hell/).

Avedon, Elliott M., and Brian Sutton-Smith. 1971. The Study of Games. New York City, N.Y.: J. Wiley.

Ball, Kirstie. 2002. "Categorizing the Workers: Electronic surveillance and social ordering in the call centre." Pp. 201-225 in Surveillance and Social Sorting: Privacy, Risk, and Automated Discrimination. London: Routledge.

Banks, John. 2009. "Co-creative Expertise: Auran Games and Fury - A case study." Media International Australia: incorporating Culture and Policy (130):77-89.

Banks, John. 2002. "Gamers as Co-creator: Enlisting the Virtual Audience--A Report from the Net Face." Pp. 188- 212 in Mobilising the Audience. University of Queensland Press.

Bartle, Richard A. 2004. Designing virtual worlds. Indianapolis, Ind.: New Riders Pub.

Bartle, Richard A. 1996. "Hearts, Clubs, Diamonds, Spades: Players Who Suit MUDS." Retrieved (http://mud.co.uk/richard/hcds.htm).

Bateman, Chris. 2010. "Sicart \& Bateman (2): Solitary Play." International Hobo. Retrieved February 2, 2011 (http://blog.ihobo.com/2010/07/sicart-bateman-2solitary-play.html).

Bauman, Zygmunt. 2000. Liquid Modernity. Oxford: Polity Press.

Begy, Jason, and Mia Consalvo. 2011. "Achievements, Motivations and Rewards in Faunasphere." Game Studies 11(1).

Benkler, Yochai. 2006. The Wealth of Networks: How social production transforms markets and freedom. New Haven CT: Yale University Press. 
Benton, Joel. 2010. "The Future of the Independent." in Generator Networking Event: Views \& Experience From Close By and Far Away. bitHeads Studio, Ottawa, Canada.

Best, J. 2001. Damned lies and statistics: untangling numbers from the media, politicians, and activists. Berkeley: University of California Press.

Bethke, Ray, Min Kin, Ray Muzyka, and Nicolay Nickelsen. 2010. "The Great MMO Hope." Retrieved

(http://audio.sxsw.com/2010/podcasts/031510i_TheGreatMMOHope.mp3).

Birke, Chris. 2011. "Ethos Before Analytics." Gamasutra. Retrieved October 5, 2011 (http://www.gamasutra.com/view/feature/6487/ethos_before_analytics.php? print=1).

Bishop, Todd. 2011. "How Valve Experiments with the Economics of Video Games." Geek Wire. Retrieved November 8, 2011

(http://www.geekwire.com/2011/experiments-video-game-economics-valvesgabe-newell).

Blair, Lucas. 2011. "The Cake Is Not a Lie: How to design effective achievements, Part 2." Gamasutra. Retrieved May 20, 2011

(http://www.gamasutra.com/view/feature/6375/the_cake_is_not_a_lie_how_to_.p hp?print=1).

Blizzard Entertainment. 2010. "Battle.net ${ }^{\circledR}$ and Facebook Integration Announced.” Retrieved July 29, 2010 (http://us.blizzard.com/enus/company/press/pressreleases.html?100505).

Bogost, Ian. 2010. "Cow Clicker: The making of obsession.” Ian Bogost - Videogame Theory, Criticism, Design. Retrieved July 22, 2010 (http://www.bogost.com/blog/cow_clicker_1.shtml).

Bogost, Ian. 2007. Persuasive Games: The expressive power of videogames. Cambridge MA: The MIT Press.

Bogost, Ian. 2006. Unit Operations: an approach to videogame criticism. Cambridge MA: The MIT Press.

Boltanski, Luc, and Eve Chiapello. 2005. "The New Spirit of Capitalism." International Journal of Politics, Culture and Society 18(3-4):161-188.

Boltanski, Luc, and Eve Chiapello. 2007. The New Spirit of Capitalism. Brooklyn NY: Verso.

Bolter, Jay David, and Richard Grusin. 1999. Remediation: Understanding new media. 
Cambridge MA: The MIT Press.

Bowker, Geoffrey C., and Susan Leigh Star. 1999. Sorting Things Out: Classification and its consequences. Cambridge MA: The MIT Press.

Brathwaite, Brenda. 2011a. "Developing Self-Discipline.” Applied Game Design. Retrieved January 17, 2011 (http://bbrathwaite.wordpress.com/2011/01/16/developing-self-discipline/).

Brathwaite, Brenda. 2011b. “Social Game Developers Rant Back.” Applied Game Design. Retrieved March 7, 2011

(http://bbrathwaite.wordpress.com/2011/03/03/gdc-2011-social-game-developersrant-back/).

Brathwaite, Brenda. 2010. "Train (or How I Dumped Electricity and Learned to Love Design).” in GDC 2010, GDC Vault. www.gdcvault.com.

Brathwaite, Brenda. 2011c. "Who Directed Star Wars?” Applied Game Design. Retrieved November 16, 2011 (http://bbrathwaite.wordpress.com/2011/11/11/who-directedstar-wars/).

Brightman, James. 2011a. "Violent Games Can Hinder Development of Empathy in Children, says Study.” Industry Gamers. Retrieved April 6, 2011 (http://www.industrygamers.com/news/violent-games-can-hinder-developmentof-empathy-in-children-says-study/).

Brightman, James. 2011b. “Zynga Rallies Against Criticism of Metrics Approach.” Industry Gamers. Retrieved March 31, 2011

(http://www.industrygamers.com/news/zynga-rallies-against-criticism-of-metricsapproach/).

Brown, Nathan. 2011. "Wilson: EA Sports Football World a 'gut-wrenching change'." Edge Magazine. Retrieved January 26, 2012 (http://www.edgeonline.com/news/wilson-ea-sports-football-world-gut-wrenching-change).

Bruno, Jose R. Gonzalez. 2010. "Tyranny of the Masses: Why developers should be weary of tracking player behavior." Game Reader. Retrieved December 17, 2010 (http://gamereader.net/2010/12/07/tyranny-of-the-masses/).

Budgen, Sebastian. 2000. "A New 'Spirit of Capitalism'.” New Left Review 1(1):149 156. Retrieved September 3, 2012.

Cadin, Loic, and Francis Guerin. 2006. "What Can We Learn from the Video Games Industry?” European Management Journal 24(4):248-255.

Caillois, Roger. 1961. Man, Play, and Games. New York City, N.Y.: Free Press of 


\section{Glencoe.}

Campbell, J. D. 2000. “'Training for Sport is Training for War': Sport and the transformation of the British Army, 1860-1914." The International Journal of the History of Sport 17(4):21 - 58.

Caoili, Eric. 2011. “66\% Of U.S. Online Population Are Casual Gamers.” Worlds in Motion.Biz. Retrieved February 7, 2011 (http://worldsinmotion.biz/2011/02/study_66_of_us_online_populati.php).

Caoili, Eric. 2010a. "Playdom's Siegel: Indies need to develop social games." Worlds in Motion.Biz. Retrieved November 12, 2010 (http://worldsinmotion.biz/2010/11/migs_2010_playdoms_siegel_indi.php\#more).

Caoili, Eric. 2010b. "Playfish's Valadares on Intuition Versus Metrics: 'Make your own decisions'." Worlds in Motion.Biz. Retrieved August 17, 2010 (http://worldsinmotion.biz/2010/08/gdc_europe_playfishs_valadares.php).

Caoili, Eric. 2010c. "Wizard101 And How Social Games Influence MMOs." Worlds in Motion.Biz. Retrieved August 13, 2010 (http://worldsinmotion.biz/2010/08/interview_wizard101_and_how_so.php).

Caoili, Eric. 2012. "Zynga Doles out Stocks to Keep Employees from Leaving." Gamasutra. Retrieved September 5, 2012 (http://www.gamasutra.com/view/news/175786/Zynga_doles_out_stocks_to_keep _employees_from_leaving_report.php).

Carmel, Ron. 2010. "Indies and Publishers: Fixing a system that never worked." in Indy Games Summit 2010. www.gdcvault.com.

Castells, Manuel. 2000. The Rise of the Network Society. Oxford; Malden, MA: Blackwell Publishers.

Castle, Lou. 2011. "Zynga's Castle On Social Game Metrics." Gamasutra. Retrieved June 22, 2011 (http://www.gamasutra.com/view/news/35373/GDC_Europe_Speaker_Spotlight_ Zyngas_Castle_On_Social_Game_Metrics.php).

Chen, Sande. 2009. "The Social Network Game Boom.” Gamasutra: The Art \& Business of Making Games Retrieved (http://www.gamasutra.com/view/feature/4009/the_social_network_game_boom. php?print=1).

Chiang, Oliver. 2011. "The Master of Online Mayhem.” Forbes. Retrieved February 14, 2011 (http://www.forbes.com/forbes/2011/0228/technology-gabe-newellvideogames-valve-online-mayhem.html). 
Clements, Matthew T., and Hiroshi Ohashi. 2004. Indirect Network Effects and the Product Cycle: Video games in the U.S., 1994-2002. NET Institute Retrieved April 5, 2011 (http://ssrn.com/abstract=500922).

Cohendet, Patrick, and Laurent Simon. 2007. "Playing Across the Playground: Paradoxes of knowledge creation in the videogame firm.” Journal of Organizational Behavior 28:587-605.

Collins, Nick. 2011. "Children Who Love Video Games Have Brains Like Gamblers." The Telegraph Retrieved November 16, 2011 (http:/www.telegraph.co.uk/science/science-news/8891651/Children-who-lovevideo-games-have-brains-like-gamblers.html).

Consalvo, Mia. 2009a. "Hardcore Casual: Game culture return(s) to Ravenhearst." Pp. 50-54 in Proceedings of the 4th International Conference on Foundations of Digital Games. New York City, N.Y.: ACM.

Consalvo, Mia. 2009b. “There is No Magic Circle." Games and Culture 4(4):408 - 417.

Consalvo, Mia, and Nathan Dutton. 2006. "Game Analysis: Developing a methodological toolkit for the qualitative study of games.” Game Studies 6(1). Retrieved (http://gamestudies.org/0601/).

Cook, Daniel. 2011a. "GDC 2011 Game of Platform Power.” Slideshare. Retrieved March 21, 2011 (http://www.slideshare.net/danctheduck/gdc-2011-game-ofplatform-power).

Cook, Daniel. 2011b. "Player Metrics Vs. The Vocal Minority.” Gamasutra. Retrieved November 21, 2011 (http://www.gamasutra.com/view/news/38205/Opinion_Player_Metrics_Vs_The_ Vocal_Minority.php).

Cook, Daniel. 2010. "Why Videogames are so Special Contrary to Other Games." Empyre Forum. Retrieved January 30, 2012 (http://lists.cofa.unsw.edu.au/pipermail/empyre/2010-December.txt).

Copier, Marinka. 2007. "Beyond the Magic Circle : A network perspective on role-play in online games.” PhD Thesis, Utrecht: Utrecht University Retrieved February 2, 2011 (http://igitur-archive.library.uu.nl/dissertations/2007-0710214621/UUindex.html).

Cortazzi, Martin. 2007. "Narrative Analysis in Ethnography.” Pp. 384 - 395 in Handbook of Ethnography, edited by Paul Atkinson, Amanda Coffey, Sara Delamont, John Lofland, and Lyn Lofland. Los Angeles CA: Sage Publications, Ltd.

Costikyan, Greg. 2009. “Mothers, Don’t Let Your Children Grow Up to Be Game 
Developers." Play This Thing. Retrieved October 6, 2009

(http://playthisthing.com/mothers-dont-let-your-children-grow-be-gamedevelopers).

Costikyan, Greg. 2010. "Social Network Games: ARPU over design." Gamasutra.

Retrieved May 3, 2010

(http://www.gamasutra.com/blogs/GregCostikyan/20100420/4977/Social_Networ k_Games_ARPU_over_Design.php).

Cover, Rob. 2006. "Gaming (Ad)diction: Discourse, identity, time and play in the production of the gamer addiction myth." Game Studies 6(1). Retrieved (http://gamestudies.org/0601/).

Crawford, Chris. 2003. Chris Crawford on Game Design. Indianapolis, Ind.: New Riders.

Crawford, Chris. 1984. The Art of Computer Game Design. New York City, N.Y.:

McGraw-Hill Retrieved June 5, 2009

(http://www.vancouver.wsu.edu/fac/peabody/game-book/Coverpage.html).

Crossley, Rob. 2011a. “Can Crowdsourcing Revolutionise Indie Funding?” Develop. Retrieved January 28, 2011 (http://www.develop-online.net/news/36880/Cancrowdsourcing-revolutionise-indie-funding).

Crossley, Rob. 2011b. "EA: Digital will rise above retail this year." Develop. Retrieved January 10, 2011 (http://www.develop-online.net/news/36709/EA-Digital-willrise-above-retail-this-year).

Crossley, Rob. 2011c. "Exhausted Insider Exposes Crunch at Kaos.” Develop. Retrieved January 17, 2011 (http://www.develop-online.net/news/36768/Exhausted-insiderexposes-crunch-at-Kaos).

Crossley, Rob. 2011d. "Game Budgets 'Have Tripled' on 3DS and PSP2.” Develop. Retrieved March 14, 2011 (http://www.develop-online.net/news/37269/Gamebudgets-have-tripled-on-3DS-and-PSP2).

Crossley, Rob. 2011e. "LA Noire studio accused of 'thankless crunch'." Develop. Retrieved June 20, 2011 (http://www.develop-online.net/news/38072/LA-Noirestudio-accused-of-thankless-crunch-work? utm_source=feedburner\&utm_medium=feed\&utm_campaign=Feed $\% 3 \bar{A}+$ developmag\%2Fifbh+\%28Develop\%29).

Crossley, Rob. 2011f. "Mobile industry to grow 15\% in 2011.” Develop. Retrieved April 8, 2011 (http://www.develop-online.net/news/37452/Analyst-Mobile-industry-togrow-15-in-2011? utm_source $=$ feedburner\&utm_medium $=$ feed\&utm_campaign $=$ Feed $\% 3 \bar{A}+$ developmag\%2Fifbh+\%28Develop\%29). 
Crossley, Rob. 2011g. "New App Store Law 'is Killing Dev Revenues'.” Develop. Retrieved June 1, 2011 (http://www.develop-online.net/news/37815/New-AppStore-law-is-killing-dev-revenues).

Crossley, Rob. 2011h. "Rumour: Android charting game-time, not installs." Develop. Retrieved April 8, 2011 (http://www.develop-online.net/news/37456/RumourAndroid-charting-game-time-not-installs).

Crossley, Rob. 2011i. "Sony: Future game tech could read your emotions.” Develop. Retrieved August 29, 2011 (http://www.develop-online.net/news/38493/SonyFuture-game-tech-could-read-your-emotions).

Crossley, Rob. 2011j. "Valve: Five interviews.” Develop. Retrieved May 17, 2011 (http://www.develop-online.net/features/1188/Valve-Five-interviews).

Cunningham, Scott, Benjamin Engelstätter, and Michael R. Ward. 2011. "Understanding the Effects of Violent Video Games on Violent Crime." SSRN eLibrary. Retrieved June 23, 2011 (http://papers.ssm.com/sol3/papers.cfm?abstract_id=1804959).

Curtis, Tom. 2012. "38 Studios, Big Huge Games Lay Off Their Entire Staff." Gamasutra. Retrieved August 28, 2012 (http://gamasutra.com/view/news/171015/38_Studios_Big_Huge_Games_lay_off _their_entire_staff.php).

Dallman, Joshua. 2011. "Redesigning Wild Ones into Playdom's Top Game: A social game design reboot." Gamasutra. Retrieved April 29, 2011 (http://www.gamasutra.com/view/feature/6362/redesigning_wild_ones_into_.php ?print=1).

David, Paul A. 1985. "Clio and the Economics of QWERTY." The American Economic Review 75(0):332-337.

Davison, John. 2010. "Too Big and Too Hard." GamePro. Retrieved May 5, 2010 (http://www.gamepro.com/article/news/215033/too-big-and-too-hard/).

Deejay. 2011. "The False Promise of Digital Distribution.” Develop. Retrieved February 18, 2011 (http://www.develop-online.net/news/37053/OPINION-The-falsepromise-of-digital-distribution).

Deleuze, Gilles. 1992. "Postscript on Societies of Control." October 59(Winter):3 - 7.

Della Rocca, Jason. 2009. "MIGS Keynote." in Montreal International Game Summit. Montréal, Canada.

Della Rocca, Jason. 2010. "Playing For Our Future.” The Escapist Retrieved June 23, 2010 (http://www.escapistmagazine.com/articles/view/issues/issue_252/7504- 
Playing-For-Our-Future).

Deterding, Sebastian. 2011. “A Quick Buck by Copy and Paste.” Gamification Research Network. Retrieved October 5, 2011 (http://gamification-research.org/2011/09/aquick-buck-by-copy-and-paste/).

Deterding, Sebastian. 2010a. "Pawned: Gamification and its discontents." in Playful 2010. Conway Hall, London Retrieved November 4, 2010 (http://www.slideshare.net/dings/pawned-gamification-and-its-discontents).

Deterding, Sebastian. 2010b. Social Game Studies: A workshop report. Hamburg: Hans Bredlow Institute for Media Research Retrieved November 4, 2010 (http://socialgamestudies.org/report).

Dibbell, Julian. 2006. Play Money: Or, how I quit my day job and made millions trading virtual loot. New York City, N.Y.: Basic Books.

Donovan, Tristan. 2010. Replay: The history of video games. East Sussex, UK: Yellow Ant.

Dormann, Claire, Jennifer R. Whitson, and Robert Biddle. 2011. "Computer Games for Affective Learning." in Handbook of Research on Improving Learning and Motivation through Educational Games: Multidisciplinary Approaches, edited by Patrick Felicia. Hershey PA: IGI Global.

Dormans, Joris. 2006. "On the Role of the Die: A brief ludologic study of pen-and-paper roleplaying games and their rules." Game Studies 6(1). Retrieved February 2, 2011 (http://gamestudies.org/0601/articles/dormans).

Drachen, Anders, and Alessandro Canossa. 2009. "Towards Gameplay Analysis via Gameplay Metrics." Pp. 202-209 in Proceedings of MindTrek2009. New York City, N.Y.: ACM Retrieved July 26, 2011.

Drachen, Anders, Alessandro Canossa, and Georgios N. Yannakakis. 2009. "Player Modeling using Self-Organization in Tomb Raider: Underworld.” in Proceedings of the 5th International Conference on Computational Intelligence and Games. IEEE.

Ducheneaut, Nicholas, Nick Yee, Eric Nickell, and Robert J. Moore. 2006. "Building an MMO With Mass Appeal: A look at gameplay in World of Warcraft." Games and Culture 1(4):281 - 317.

Ducheneaut, Nicholas, Nick Yee, Eric Nickell, and Robert J. Moore. 2007. "The Life and Death of Online Gaming Communities: A look at guilds in World of Warcraft." Pp. 839 - 848 in CHI Proceedings. San Jose, California. 
Dyer, Mitch. 2011. "12 Game Studios That Died in 2011." IGN. Retrieved August 28, 2012 (http://ca.ign.com/articles/2011/12/01/12-game-studios-that-died-in-2011).

Dyer-Witheford, Nick, and Greig de Peuter. 2006. "'EA Spouse' and the Crisis of Video Game Labour: Enjoyment, exclusion, exploitation, exodus." Canadian Journal of Communication 31:599-617.

Dyer-Witheford, Nick, and Greig de Peuter. 2009. Games of Empire: Global capitalism and video games. Minneapolis MN: University of Minnesota Press.

Eco, Umberto. 2010. "SPIEGEL Interview with Umberto Eco: 'We Like Lists Because We Don't Want to Die'." Spiegel Online. Retrieved November 30, 2010 (http://www.spiegel.de/international/zeitgeist/0,1518,659577,00.html).

Edery, David, and Ethan Mollick. 2009. Changing the Game. Upper Saddle River, New Jersey: FT Press.

Edwards, P.N. 1996. "The Closed World: Computers and the politics of discourse in Cold War America."

Electronic Entertainment Design and Research. 2011. "Alternative Business Models with DLG \& DLC." www.eedar.com. Retrieved March 23, 2011 (http://www.gamasutra.com/view/news/33648/EEDAR_55_Of_Xbox_360_Owne rs_Purchased_A_Downloadable_Game_In_2010.php).

Elliott, Phil. 2011a. "Alan Miller: Part 1 Interview." gamesindustry.biz. Retrieved February 18, 2011 (http://www.gamesindustry.biz/articles/2011-02-16-alan-millerinterview).

Elliott, Phil. 2011b. "Justin Johnson Interview.” gamesindustry.biz. Retrieved March 2, 2011 (http://www.gamesindustry.biz/articles/2011-02-23-justin-johnsoninterview).

Elliott, Phil. 2010. "Metrics the Key to Successful Social Game Design." gamesindustry.biz. Retrieved July 28, 2010 (http://www.gamesindustry.biz/articles/metrics-the-key-to-successful-socialgame-design-zynga).

Elliott, Phil. 2011c. "XBLA Publishing 'Massively' Harder Now - Brown.” gamesindustry.biz. Retrieved July 28, 2011 (http://www.gamesindustry.biz/articles/xbla-publishing-massively-harder-nowbrown).

Entertainment Software Association. 2010. Essential Facts about the Computer and Video Game Industry: 2010 sales, demographic and usage data. Retrieved August 4, 2011 (http://www.theesa.com/). 
Ericson, Richard Victor, Aaron Doyle, and Dean Barry. 2003. Insurance as Governance. Toronto: University of Toronto Press.

Emkvist, Mirko, and Patrik Ström. 2008. "Enmeshed in Games with the Government: Governmental policies and the development of the Chinese online game industry." Games and Culture 3(1):98 - 126.

Ernkvist, Mirko, and Patrik Ström. 2006. "Playing the Franchise Game: A comparison of the competitiveness of the Japanese and US independent video game software firms, 1995-2005." Scandinavian Consortium for Organizational Research Retrieved (http://www.hgu.gu.se/Files/ekonomisk_historia/Christer_Lundh/HS_vt08/Playin g\%20the\%20franchise\%20game.pdf).

Evans, Jon. 2010. "Dear Foursquare, Gowalla: Please let's stop pretending this is fun." TechCrunch. Retrieved November 15, 2010 (http://techcrunch.com/2010/11/13/foursquare-gowalla-stop-pretending-fun/).

Fahey, Mike. 2011. "Dragon Age II Dev Rates His Own Game On Metacritic, EA Bets Obama Voted For Himself Too.” Kotaku. Retrieved March 15, 2011 (http://kotaku.com/\#!5782097/dragon-age-ii-dev-rates-his-own-game-onmetacritic-ea-bets-obama-voted-for-himself-too).

Fahey, Rob. 2011a. "Beachside Burnout.” gamesindustry.biz. Retrieved July 8, 2011 (http://www.gamesindustry.biz/articles/2011-07-08-beachside-burnout-editorial).

Fahey, Rob. 2010. "Game of Life." gamesindustry.biz. Retrieved August 6, 2010 (http://www.gamesindustry.biz/articles/2010-08-06-game-of-life-editorial? page=1).

Fahey, Rob. 2011b. "Rock and a Hard Place." gamesindustry.biz. Retrieved May 6, 2011 (http://www.gamesindustry.biz/articles/2011-05-06-rock-and-a-hard-placeeditorial).

Fahey, Rob. 2012. "The End of the Console Era?” Gamesindustry International.

Retrieved June 22, 2012 (http://www.gamesindustry.biz/articles/2012-06-22-theend-of-the-console-era).

Feenberg, Andrew. 2002. Transforming Technology: A critical theory revisited. New York City, N.Y.: Oxford University Press.

Fenn, Jackie, and Mark Raskino. 2008. Mastering the Hype Cycle: How to choose the right innovation at the right time. Boston MA: Harvard Business School Press.

Flanagan, Mary. 2009. Critical Play: Radical game design. Cambridge MA: The MIT Press. 
Foucault, Michel. 1991. The Foucault Effect: Studies in governmentality. Chicago IL: University of Chicago Press.

Foucault, Michel. 1988. The History of Sexuality: The care of the self. New York City, N.Y.: Vintage Books.

Frasca, Gonzalo. 1999. "Ludology Meets Narratology: Similtude and differences between (video)games and narrative." Parnasso 3. Retrieved February 7, 2011 (http://www.ludology.org/articles/ludology.htm).

Frasca, Gonzalo. 2001. "Videogames of the Oppressed: Videogames as a means for critical thinking and debate." Master of Information Design and Technology, Atlanta, GA: Georgia Institute of Technology.

Fullerton, Tracy. 2008. Game Design Workshop: A playcentric approach to creating innovative games. 2nd ed. Burlington MA: Morgan Kaufmann Publishers.

Funcom. 2012. "Funcom Reduces Operational Costs Following 'The Secret World' Launch." www.funcom.com. Retrieved August 28, 2012 (http://www.funcom.com/news/funcom_reduces_operational_costs_following_the _secret_world_launch).

Galloway, Alexander R. 2004. Protocol: How control exists after decentralization. Cambridge MA: The MIT Press.

Galloway, A.R. 2006. Gaming: Essays on algorithmic culture. Minneapolis MN: University of Minnesota Press.

Garland, David. 1991. Punishment and Modern Society: A study in social theory. Oxford: Oxford University Press.

Gasse, Nicholas. 2011. "Fan Funding." gamesindustry.biz. Retrieved April 18, 2011 (http://www.gamesindustry.biz/articles/2011-04-18-fan-funding-article?page=1).

Gaudiosi, John. 2011. 'Mobile Will Become 'The Console of The Future,' says Developer.” Industry Gamers. Retrieved October 24, 2011 (http://www.industrygamers.com/news/mobile-will-become-the-console-of-thefuture-says-developer/).

Gee, James Paul. 2007. What Video Games Have to Teach Us About Learning and Literacy. 2007th ed. New York City, N.Y.: Palgrave Macmillan.

Geertz, Clifford. 1973. The Interpretation Of Cultures. New York City, N.Y.: Basic Books.

Gibson, Rick. 2010. "Do We Still Need Publishers in an Online World?" Develop 
(104):12-16.

Gibson, Rick. 2011. "The Problem with Game Analytics." Develop. Retrieved March 10, 2011 (http://www.develop-online.net/news/37244/OPINION-The-problem-withgame-analytics).

Giles, Jim. 2005. “Internet Encyclopaedias go Head to Head.” Nature 438:900 - 910. Retrieved September 22, 2010.

Goldberg, Harold. 2011. All Your Base Are Belong to Us: How fifty years of videogames conquered pop culture. New York City, N.Y.: Three Rivers Press.

Gouglas, Sean et al. 2010. Computer Games and Canada's Digital Economy: The role of universities in promoting innovation. SSHRC Retrieved

(http://ra.tapor.ualberta.ca/ circa/wpcontent/uploads/2010/03/ComputerGamesAndCanadasDigitalEconomy1.pdf).

Graft, Kris. 2010. "Around The Block With Brian Fargo.” Gamasutra. Retrieved January 17,2011

(http://www.gamasutra.com/view/feature/6256/around_the_block_with_brian_far go.php).

Graft, Kris. 2011a. "How Shaba’s Ex-Boss Escaped From The 'Great Middle'.”

Gamasutra. Retrieved May 27, 2011

(http://www.gamasutra.com/view/news/34798/Interview_How_Shaba_ExBoss_E scaped_From_The_Great_Middle.php).

Graft, Kris. 2011b. "Iwata: Dev costs for high-end 3DS games may approach Wii costs." Gamasutra. Retrieved July 28, 2011

(http://www.gamasutra.com/view/news/29067/Iwata_Dev_Costs_For_HighEnd_3 DS_Games_May_Approach_Wii_Costs.php).

Graft, Kris. 2011c. "Tretton: Platforms must have 'cutting edge' tech at launch to stay relevant." Gamasutra. Retrieved April 8, 2011 (http://www.gamasutra.com/view/news/34012/Tretton_Platforms_Must_Have_Cu tting_Edge_Tech_At_Launch_To_Stay_Relevant.php).

Greenberg, Saul, and Bill Buxton. 2008. "Usability Evaluation Considered Harmful (Some of the Time).” Pp. $111-120$ in CHI 2008 Proceedings. New York City, N.Y.: ACM.

Grimes, Sara M., and Andrew Feenberg. 2009. "Rationalizing Play: A critical theory of digital gaming.” The Information Society 25:105 - 118.

Hacking, Ian. 1990. The Taming of Chance. Cambridge, UK: Cambridge University Press. 
Haggerty, Kevin D. 2001. Making Crime Count. Toronto, Canada: University of Toronto Press.

Handrahan, Matthew. 2011a. "Freemium: Is it evil?" gamesindustry.biz. Retrieved July 28, 2011 (http://www.gamesindustry.biz/articles/2011-07-26-free-is-it-evil-blogentry).

Handrahan, Matthew. 2011b. "Rise in XBLA Prices Bucking Digital Trend." gamesindustry.biz. Retrieved July 29, 2011 (http://www.gamesindustry.biz/articles/2011-07-22-average-price-of-xbla-gamescreeping-up).

Hardt, Michael, and Antonio Negri. 2001. Empire. Boston, MA: Harvard University Press.

Hawkins, Trip. 2010. "The Movie: Fact and fiction.” Digital Chocolate. Retrieved October 13, 2010 (http://blog.digitalchocolate.com/?p=656).

Head, Alison J., and Michael B. Eisenberg. 2010. "How Today's College Students use Wikipedia for Course-related Research.” First monday 15(3). Retrieved March 18,2010 (http://www.uic.edu/htbin/cgiwrap/bin/ojs/index.php/fm/article/view/2830/2476).

Hecker, Chris. 2010. “Achievements Considered Harmful?” in GDC 2010, GDC Vault. www.gdcvault.com Retrieved May 10, 2010 (http://www.gamasutra.com/view/news/27646/GDC_Heckers_Nightmare_Scenari o_A_Future_Of_Rewarding_Players_For_Dull_Tasks.php).

Herman, Leonard. 1997. Phoenix: The fall and rise of video games. 2nd ed. Springfield, NJ: Rolenta Press.

Herz, J. C. 1997. Joystick Nation: How videogames ate our quarters, won our hearts, and rewired our minds. New York City, N.Y.: Little Brown and Company.

Holtman, Jason. 2009. "Games Entertainment in the Age of Connectivity." in Montreal International Game Summit. Montréal, Canada.

Huff, Darrell, and Irving Geis. 1954. How to Lie with Statistics. New York City, N.Y.: Norton.

Huizinga, Johan. 1955. Homo Ludens: A study of the play-element in culture. Boston MA: Beacon Press.

Huling, Ray. 2010. "Gamification: Turning work into play." $h+$. Retrieved September 10, 2010 (http://www.hplusmagazine.com/articles/art-entertainment/gamificationturning-work-play). 
Humphreys, Sal. 2005. "Productive Players: Online computer games' challenge to conventional media forms." Communication and Critical/Cultural Studies 2(1):37 -51 .

Humphreys, Sal. 2008. "Ruling the Virtual World: Governance in massively multiplayer online games." European Journal of Cultural Studies 11(2):149 - 171.

Humphreys, Sal. 2007. “'You're In Our World Now.' TM: Ownership and access in the proprietary community of an MMOG."Pp. 76-96 in Information Communication Technologies and Emerging Business Strategies, edited by S. Van Der Graaf and Y. Washida. London: Idea Group Publishing.

Hyman, Paul. 2007. "For Better or Worse: A quality of life update." Game Developer Magazine (June/July):7-11.

Hyman, Paul. 2010. “Targeted Focus, Broad Audience?” Gamasutra. Retrieved June 8, 2010

(http://www.gamasutra.com/view/feature/5829/targeted_focus_broad_audience.ph p).

International Game Developers Association. 2004. "Quality of Life White Paper.” Retrieved May 5, 2011 (http://www.igda.org/quality-life-white-paper-info).

Irwin, Mary Jan. 2008. “Cooking Up A Blockbuster Game.” Forbes. Retrieved July 15, 2012 (http://www.forbes.com/2008/11/21/games-eedar-developers-tech-ebizcx_mji_1121eedar.html).

Jackson, Steven J., Paul N. Edwards, Geoffrey C. Bowker, and Cory P. Knobel. 2007. "Understanding Infrastructure: History, heuristics, and cyberinfrastructure policy." First monday 12(6). Retrieved (http://firstmonday.org/issues/issue12_6/jackson/index.html).

Järvinen, Aki. 2011. "Book Project on Hold." Games for Social Networks: Notes On The Design and Business of Networked Play. Retrieved February 1, 2011 (http://games4networks.posterous.com/book-project-on-on-hold).

Järvinen, Aki. 2010. "My Working Definition of \#socialgames." Games for Social Networks: Notes On The Design and Business of Networked Play. Retrieved July 21, 2010 (http://games4networks.posterous.com/my-working-definition-ofsocialgames).

Jayson, Sharon. 2011. "Don't study the video game, study the player." USA Today. Retrieved September 18, 2011 (http://yourlife.usatoday.com/health/story/2011-0914/Dont-study-the-video-game-study-the-player/50406018/1 ?source=twitter).

Jenkins, Henry. 1999. Congressional Testimony on Media Violence. Washington, D.C. 
Retrieved (http://web.mit.edu/comm-forum/papers/jenkins_ct.html).

Jenkins, Henry. 2006. Convergence Culture: Where old and new media collide. New York City, N.Y.: New York University Press.

Johns, Jennifer. 2006. "Video Games Production Networks: Value capture, power relations and embeddedness." Journal of Economic Geography 6:151-180.

Johnson, Soren. 2010. "Fear and Loathing in Farmville." Designer Notes. Retrieved March 22, 2010 (http://www.designer-notes.com/?p=195).

Johnson, Soren. 2009. "The Case for Metacritic.” Designer Notes. Retrieved January 26, 2012 (http://www.designer-notes.com/?p=130).

Johnson, Steven B. 2005. Everything Bad is Good for You: How today's popular culture is actually making us smarter. New York City, N.Y.: Riverhead Books.

Jones, Steven E., and George K. Thiruvathukal. 2012. Codename Revolution: The Nintendo Wii Platform. Cambridge MA: The MIT Press.

Juul, Jesper. 2010. A Casual Revolution: Reinventing video games and their players. Cambridge MA: The MIT Press.

Juul, Jesper. 2005. Half-Real. Cambridge MA: The MIT Press.

Kelly, Tadhg. 2010. "Fight Design Bloat with Data." Gamasutra. Retrieved September 16,2010 (http://www.gamasutra.com/blogs/TadhgKelly/20100910/5803/Fight_Design_Blo at_with_Data.php).

Kent, Steven L. 2001. The Ultimate History of Video Games: From Pong to Pokemon. New York City, N.Y.: Three Rivers Press.

Kerr, Aphra. 2006. The Business and Culture of Digital Games: Gamework and gameplay. London, UK: Sage Publications Ltd.

Kim, Amy Jo. 2000. Community Building on the Web : Secret strategies for successful online communities. Berkeley, CA: Peachpit Press.

Kim, Amy Jo. 2010. "MetaGame Design: Reward systems that drive engagement." in Social Games Summit at GDC 2010. Retrieved (www.gdcvault.com).

Kim, Amy Jo. 2009. "Putting the Fun in Functional: Applying game mechanics to functional software." in Google TechTalks. Retrieved (http://www.youtube.com/watch?v=ihUt-163gZI). 
Kim, Jun H. et al. 2008. "Tracking Real-Time User Experience (TRUE): A comprehensive instrumentation solution for complex systems.” Pp. $443-451$ in CHI 2008 Proceedings. New York City, N.Y.: ACM.

Kline, Stephen, Nick Dyer-Witheford, and Greig de Peuter. 2003. Digital Play: The interaction of technology, culture, and marketing. Montréal: McGill-Queen's University Press.

Knox, Hannah, Mike Savage, and Penny Harvey. 2006. "Social networks and the study of relations: networks as method, metaphor and form." Economy and Society 35(1):113-140.

Kohler, Chris. 2010. "Farm Wars: How Facebook games harvest big bucks." Wired. Retrieved July 28, 2010 (http://www.wired.com/gamelife/2010/05/farmwars/all/1).

Koster, Raph. 2002. “A Philosophical Statement on Playerkilling.” Retrieved (http://www.raphkoster.com/gaming/pkphilosophy.shtml).

Koster, Raph. 2005. A Theory of Fun for Game Design. Scottsdale, AZ: Paraglyph Press.

Koster, Raph. 2010. “Gameifying Everything.” Raph Koster's Website. Retrieved September 14, 2010 (http://www.raphkoster.com/2010/02/18/gameifyingeverything/).

Kücklich, Julian. 2005. "Precarious Playbour: Modders and the digital games industry." fibreculture (5). Retrieved February 19, 2009

(http://journal.fibreculture.org/issue5/kucklich.html).

Kücklich, Julian. 2009. "Virtual Worlds and Their Discontents: Precarious sovereignty, governmentality, and the ideology of play." Games and Culture 4(4):340 - 352.

Kultima, Annakaisa. 2009. "Casual Game Design Values.” Pp. 58-65 in Proceedings of MindTrek2009. New York City, N.Y.: ACM.

Lai, Albert. 2011. "7 Top Social Game Metrics for 2011: The A.R.M metrics framework." Slideshare. Retrieved March 16, 2011

(http://www.slideshare.net/kontagent/kontagent-top-7-deadly-arms-metrics-forsocial-games).

Lanier, Jaron. 2010. You Are Not a Gadget: A Manifesto. New York City, N.Y.: Random House.

Latour, Bruno. 2007. "Beware, Your Imagination Leaves Digital Traces.” Times Higher Literary Supplement Retrieved (www.bruno-latour.fr/sites/default/files/P-129THES-GB.pdf). 
Latour, Bruno. 2005. Reassembling the Social: An introduction to actor-network-theory. Oxford: Oxford University Press.

Laurel, B. 2001. Utopian entrepreneur. Cambridge, Mass.: MIT Press.

Laurel, Brenda. 1991. Computers as Theatre. Reading, MA: Addison-Wesley Pub.

Lave, Jean, and Etienne Wenger. 1991. Situated Learning: Legitimate Peripheral Participation. Cambridge, UK: Cambridge University Press.

Lehdonvirta, Vili. 2010. "Virtual Worlds Don't Exist: Questioning the dichotomous approach in MMO studies." Game Studies 10(1). Retrieved August 23, 2012 (http://gamestudies.org/1001/articles/lehdonvirta).

Lessig, Lawrence. 1999. Code and Other Laws of Cyberspace. New York City, N.Y.: Basic Books.

Losh, Elizabeth. 2008. "In Polite Company: Rules of Play in Five Facebook Games." Yokohama, Japan: ACM.

Lovell, Nicholas. 2011a. "Does Zynga Intentionally Kick Players Out of its Games After Two Months?” GAMESbrief. Retrieved June 6, 2011 (http://www.gamesbrief.com/2011/06/does-zynga-intentionally-kick-players-outof-its-games-after-two-months).

Lovell, Nicholas. 2010. "Four Reasons Why VCs Won't Fund Game Companies." Gamasutra. Retrieved July 28, 2010 (http://www.gamasutra.com/blogs/NicholasLovell/20100324/4758/Four_Reasons _Why_VCs_Wont_Fund_Game_Companies.php).

Lovell, Nicholas. 2011b. "Whales, Dolphins and Minnows: The beating heart of a free-toplay game." GAMESbrief. Retrieved November 21, 2011

(http://www.gamesbrief.com/2011/11/whales-dolphins-and-minnows-the-beatingheart-of-a-free-to-play-game).

Luscombe, Belinda. 2009. “Are You Getting Scammed by Facebook Games?” Time Retrieved May 11, 2010

(http://www.time.com/time/business/article/0,8599,1935698,00.html).

Lyons, Daniel. 2009. “Is Facebook a Paradise for Scammers?” Newsweek Retrieved May 11, 2010 (http://blog.newsweek.com/blogs/techtonicshifts/archive/2009/11/06/isfacebook-a-paradise-for-scammers.aspx).

MacKinnon, Richard. 1997. "Punishing the Persona: Correctional strategies for the virtual offender." Pp. 206 - 235 in Virtual Culture: Identity and Communication in Cybersociety. London: Sage Publications. 
Maher, Jimmy. 2012. The Future Was Here: The Commodore Amiga. Cambridge MA: The MIT Press.

Malaby, Thomas. 2009. Making Virtual Worlds: Linden Lab and Second Life. Ithaca NY: Cornell University Press.

Mangalindan, J. P. 2010. "Play to Win: The game-based economy." Fortune. Retrieved September 9, 2010 (http://tech.fortune.cnn.com/2010/09/03/the-game-basedeconomy/).

Martin, Matt. 2011a. “OnLive’s Steve Perlman Interview.” gamesindustry.biz. Retrieved June 27, 2011 (http://www.gamesindustry.biz/articles/2011-06-27-onlives-steveperlman-interview).

Martin, Matt. 2011b. "The Hard Cell Interview.” gamesindustry.biz. Retrieved February 28, 2011 (http://www.gamesindustry.biz/articles/2011-02-22-the-hard-cellinterview).

Marx, G.T. 2003. "A Tack in the Shoe: Neutralizing and Resisting the New Surveillance." Journal of Social Issues 59(2):369 - 390.

Mateos-Garcia, Juan, Andrew Grantham, Georgina Voss, Ed Steinmueller, and Jonathan Sapsed. 2010. "Sticking to Their Guns: The impact of the culture and organisational practices of video games studios on the technological trajectory of the console games sector.” in Opening Up Innovation: Strategy, Organization and Technology. London, UK Retrieved (http://eprints.brighton.ac.uk/7446/).

McDevitt, Darby. 2010. “The Deaths Of Game Narrative.” Gamasutra. Retrieved August 5,2010 (http://www.gamasutra.com/view/feature/5952/the_deaths_of_game_narrative.ph p).

McGonigal, Jane. 2011. Reality Is Broken: Why games make us better and how they can change the world. London, UK: Penguin Press.

Medler, Ben. 2009. "Generations of Game Analytics, Achievements and High Scores." Eludamos 3(2):177-194.

Medler, Ben, Michael John, and Jeff Lane. 2011. "Data Cracker: Developing a visual game analytic tool for analyzing online gameplay.” in Proceedings of CHI 2011. New York City, N.Y.: ACM.

Medler, Ben, and Brian Magerko. 2011. "Analytics of Play: Using information visualization and gameplay practices for visualizing video game data." Parsons Journal for Information Mapping 3(1):1-12. 
Meer, Alec. 2011a. “Deathmapping: Just Cause 2's 11m casualties.” Rock Paper Shotgun. Retrieved May 27, 2011 (http://www.rockpapershotgun.com/2011/05/27/justcause-2-deaths-video/).

Meer, Alec. 2011b. "Indie Godfather Cliff Harris Interview." gamesindustry.biz. Retrieved January 18, 2011 (http://www.gamesindustry.biz/articles/2011-01-18indie-godfather-cliff-harris-interview).

Meer, Alec. 2011c. "LOTRO revenues up 3x since free to play switch." Retrieved January 7, 2011 (http://www.gamesindustry.biz/articles/2011-01-07-lotrorevenues-up-3x-since-free-to-play-switch).

Meer, Alec. 2011d. “Mode 7's Paul Taylor Interview.” gamesindustry.biz. Retrieved July 12, 2011 (http://www.gamesindustry.biz/articles/2011-07-12-mode-7s-paul-taylorinterview).

Meer, Alec. 2010a. "PopCap: This is 'The beginning of the end' of Facebook's 'Golden Era'." gamesindustry.biz. Retrieved October 13, 2010 (http://www.gamesindustry.biz/articles/2010-09-06-popcap-this-is-the-beginningof-the-end-of-facebooks-golden-era).

Meer, Alec. 2011e. "Puppy Dog Tales Interview." gamesindustry.biz. Retrieved April 4, 2011 (http://www.gamesindustry.biz/articles/2011-04-04-puppy-dogs-talesinterview).

Meer, Alec. 2010b. "Zynga Valued at \$5.51bn." gamesindustry.biz. Retrieved October 26, 2010 (http://www.gamesindustry.biz/articles/2010-10-26-zynga-valued-at-USD5$51 \mathrm{bn})$.

Merel, Tim. 2011. “Money Games: Online, mobile, China and more.” gamesindustry.biz. Retrieved March 14, 2011 (http://www.gamesindustry.biz/articles/2011-03-10money-games-online-mobile-china-and-more-article?page=1).

Montfort, Nick, and Ian Bogost. 2009. Racing the Beam: The Atari video computer system. Cambridge MA: The MIT Press.

Morningstar, Chip, and F. Randall Farmer. 1991. "The Lessons of Lucasfilm's Habitat." Pp. 273 - 301 in Cyberspace: First Steps. Cambridge, Massachusetts: The MIT Press.

Morrison, Chris. 2010a. “Inside a Small Developer's Success on Facebook: 5th Planet's Dawn of the Dragons.” Inside Social Games. Retrieved July 21, 2010 (http://www.insidesocialgames.com/2010/07/21/dawn-of-the-dragons-facebookgame-5th-plane).

Morrison, Chris. 2010b. "The Future Looks Bright for Small Social Game Developers on 
Facebook." Inside Social Games. Retrieved September 28, 2010 (http://www.insidesocialgames.com/2010/09/28/the-future-looks-bright-for-smalldevelopers-on-facebook).

Moura, Dinara, Magy Seif el-Nasr, and Christopher D. Shaw. 2011. "Visualizing and Understanding Players' Behavior in Video Games.” Pp. 11-15 in Proceedings of the 2011 ACM SIGGRAPH Symposium on Video Games. New York City, N.Y.: ACM.

Murray, Janet Horowitz. 1997. Hamlet on the Holodeck: The future of narrative in cyberspace. New York City, N.Y.: Free Press.

Newman, Ryan, and Brandon Sheffield. 2011. "Tenth Annual Salary Survey.” Game Developer Magazine (April): 7 -13.

Nieborg, David B. 2011. "Triple--A: The political economy of the blockbuster video game." PhD Thesis, Amsterdam, Netherlands: University of Amsterdam.

Ninja Metrics. 2011. “Katana Engine and Game Analytics Software.” Retrieved January 19, 2012 (http://ninjametrics.com/).

Nutt, Christian. 2011a. “A Philosophy That Extends Eastward: Social games Zyngastyle." Gamasutra. Retrieved February 7, 2011 (http://www.gamasutra.com/view/feature/6280/a_philosophy_that_extends_.php).

Nutt, Christian. 2011b. "EVE Online: The next steps." Gamasutra. Retrieved March 14, 2011

(http://www.gamasutra.com/view/feature/6302/eve_online_the_next_steps.php? print=1).

Nutt, Christian. 2011c. "Perfecting The Free-To-Play Battlefield Heroes." Gamasutra. Retrieved March 7, 2011 (http://www.gamasutra.com/view/news/33371/GDC_2011_Perfecting_The_FreeT oPlay_Battlefield_Heroes.php).

Nutt, Christian. 2011d. "Schafer Admits Fantasy Of Flatulence On Youth." Gamasutra. Retrieved February 14, 2011 (http://www.gamasutra.com/view/feature/6284/schafer_admits_fantasy_of_.php? print=1).

Nutt, Christian. 2010. “Social the CrowdStar Way.” Gamasutra. Retrieved November 15, 2010 (http://www.gamasutra.com/view/feature/6204/social_the_crowdstar_way.php? print=1).

Nutt, Christian. 2011e. "The Secrets Of Wooga’s Social Game Success.” Gamasutra. 
Retrieved October 5, 2011

(http://www.gamasutra.com/view/feature/6488/interview_the_secrets_of_woogas _.php?print=1).

O'Donnell, Casey. 2009. "The Everyday Lives of Video Game Developers:

Experimentally understanding underlying systems/structures.” Transformative

Works and Cultures 2(March). Retrieved March 30, 2009

(http://journal.transformativeworks.org/index.php/twc/article/view/73/76).

O’Donnell, Casey. 2008. “The Work/Play of the Interactive New Economy: Video game development in the United States and India.” PhD Thesis, New York: Rensselaer Polytechnic Institute.

Olivarez-Giles, Nathan. 2011. "Facebook F8: Redesigning and hitting 800 million users." L.A. Times, online Retrieved January 9, 2012 (http://latimesblogs.latimes.com/technology/2011/09/facebook-f8-mediafeatures.html).

Orland, Kyle. 2011a. "Facebook Changes Method For Measuring Active App Users."

Gamasutra. Retrieved January 24, 2012

(http://www.gamasutra.com/view/news/37921/Facebook_Changes_Method_For_ Measuring_Active_App_Users.php).

Orland, Kyle. 2011b. "Minecraft Draws Over \$33 Million In Revenue From 1.8M Paying Customers." Gamasutra. Retrieved April 8, 2011

(http://www.gamasutra.com/view/news/33961/Minecraft_Draws_Over_33_Millio n_In_Revenue_From_18M_Paying_Customers.php).

Orland, Kyle. 2011c. "New Research Suggests Potential Causes For 'Problem Gaming'." Gamasutra. Retrieved February 7, 2011

(http://www.gamasutra.com/view/news/32853/New_Research_Suggests_Potential _Causes_For_Problem_Gaming.php?

utm_source=feedburner\&utm_medium =feed\&utm_campaign=Feed

$\% 3 \mathrm{~A}+$ GamasutraNews+\%28Gamasutra+News\%29).

Orland, Kyle. 2011d. “Zynga Stock To Be Listed On NASDAQ.” Gamasutra. Retrieved October 17, 2011

(http://www.gamasutra.com/view/news/37898/Zynga_Stock_To_Be_Listed_On_ NASDAQ.php).

Parkin, Simon. 2010a. "BioWare’s Zeschuk Calls AAA Development 'A Poor Goal'

Today.” Gamasutra. Retrieved July 14, 2010

(http://www.gamasutra.com/view/news/29421/Develop_BioWares_Zeschuk_Call s_AAA_Development_A_Poor_Goal_Today.php).

Parkin, Simon. 2010b. “Double Fine's Schafer On 'Amnesia Fortnights' And The Pitfalls 
Of AAA." Gamasutra. Retrieved July 15, 2010

(http://www.gamasutra.com/view/news/29450/Develop_Double_Fines_Schafer_ On_Amnesia_Fortnights_And_The_Pitfalls_Of_AAA.php).

Pearce, Celia, and Artemesia. 2009. Communities of Play: Emergent cultures in multiplayer games and virtual worlds. Cambridge MA: The MIT Press.

Pearson, Dan. 2011. "You Had Me At Hello Interview.” gamesindustry.biz. Retrieved July 27, 2011 (http://www.gamesindustry.biz/articles/2011-07-25-hello-games-seanmurray-interview?page=2).

Petrillo, Fabio, Marcelo Pimenta, Francisco Trindade, and Carlos Dietrich. 2009. "What Went Wrong? A survey of problems in game development." ACM Computers in Entertainment 7(1):1-22.

Phillips, Bruce. 2009. "Staying Power: Rethinking feedback to keep players in the game." Gamasutra. Retrieved November 28, 2011

(http://www.gamasutra.com/view/feature/4171/staying_power_rethinking_feedba ck_php?print=1).

Plunkett, Luke. 2012. "Every Game Studio That's Closed Down Since 2006." Kotaku. Retrieved August 28, 2012 (http://kotaku.com/5876693/every-game-studio-thatsclosed-down-since-2006).

Plunkett, Luke. 2011. "Nintendo 3DS Gets Sudden, Massive Price Drop.” Kotaku. Retrieved July 28, 2011 (http://kotaku.com/5825495/nintendo-3ds-gets-suddenmassive-price-drop).

Poole, Steven. 2000. Trigger Happy: Videogames and the entertainment revolution. New York City, N.Y.: Arcade Publishing.

Poovey, Mary. 1998. A History of the Modern Fact: Problems of knowledge in the sciences of wealth and society. Chicago, Ill.: University Of Chicago Press.

Poppendieck, Mary, and Tom Poppendieck. 2009. Leading Lean Software Development: Results are not the point. 1st ed. Upper Saddle River, NJ: Addison-Wesley Professional.

Porter, Theodore M. 1996. Trust in Numbers. Princeton, N.J.: Princeton University Press.

Postigo, Hector. 2003. "From Pong to Planet Quake: Post-industrial transitions from leisure to work." Information, Communication \& Society 6(4):593-607.

Priebatsch, Seth. 2010. "The Game Layer on Top of the World.” Retrieved September 9, 2010 (http://tedxboston.org/videos/176-seth-priebatsch-the-game-layer-on-top-ofthe-world). 
Pruett, Chris. 2010. "Hot Failure: Tuning gameplay with simple player metrics." Gamasutra. Retrieved December 16, 2010 (http://www.gamasutra.com/view/feature/6155/hot_failure_tuning_gameplay_wit h_.php?print=1).

Purvis, T., and A. Hunt. 1993. "Discourse, ideology, discourse, ideology, discourse, ideology.” The British Journal of Sociology 44(3):473 - 499.

Radd, David. 2011a. "NPD: Number of children gamers increasing." Industry Gamers. Retrieved October 12, 2011 (http://www.industrygamers.com/news/npd-numberof-children-gamers-increasing/).

Radd, David. 2011b. “PC Gaming: Death and rebirth.” Industry Gamers. Retrieved November 21, 2011 (http://www.industrygamers.com/news/pc-gaming-death-andrebirth/).

Reader, Pauline. 2010. “Monetizing Social Games.” Proceedings of GDC 2010 in GDC Vault. Retrieved January 26, 2012 (http://www.gdcvault.com/play/1013731/Monetizing-Social).

Readman, Jeff, and Andrew Grantham. 2006. "Shopping for Buyers of Product Development Expertise: How video games developers stay ahead." European Management Journal 24(4):256-269.

Reeves, Byron, and J. Leighton Read. 2009. Total Engagement: Using games and virtual worlds to change the way people work and businesses compete. Boston MA: Harvard Business Press.

Remo, Chris. 2010a. "Controversy Erupts Over Rockstar San Diego Employee Allegations." Gamasutra. Retrieved December 16, 2010 (http://www.gamasutra.com/view/news/26803/Controversy_Erupts_Over_Rockst ar_San_Diego_Employee_Allegations.php).

Remo, Chris. 2010b. "Developers Claim 13 Weeks Of Crunch Per Year.” Gamasutra. Retrieved June 2, 2010 (http://www.gamasutra.com/view/news/28669/Study_Developers_Claim_13_Wee ks_Of_Crunch_Per_Year.php).

Ries, Eric. 2011. The Lean Startup: How today's entrepreneurs use continuous innovation to create radically successful businesses. New York City, N.Y.: Crown Business.

Robertson, Margaret. 2010. "Can't Play, Won't Play.” Hide \& Seek: Inventing new kinds of play. Retrieved November 4, 2010 (http://www.hideandseek.net/cant-playwont-play/). 
Robinson, Erin. 2011. "The Top 10 Weird Children Of Video Games and Neuroscience." Gamasutra. Retrieved August 29, 2011

(http://www.gamasutra.com/view/feature/6466/the_top_10_weird_children_of_vi deo_.php?print=1).

Robinson, William. 2012. "The Aesthetics of Play: Creativity and Art in Videogame

Performance.” Masters Thesis, Montréal, Canada: Concordia University.

Rose, Mike. 2011a. “Nintendo 'Not Interested' In Free-To-Play Model.” Gamasutra.

Retrieved June 16, 2011

(http://www.gamasutra.com/view/news/35276/Nintendo_Not_Interested_In_Free ToPlay_Model.php).

Rose, Mike. 2011b. "Sony Reveals \$20M Investment Plan For PSN-Exclusive Titles." Gamasutra. Retrieved July 14, 2011

(http://www.gamasutra.com/view/news/35840/Sony_Reveals_20M_Investment_P lan_For_PSNExclusive_Titles.php).

Rose, Mike. 2011c. "Steam Revenue For Zeboyd Games RPGs Outpaces Lifetime Xbox Mark In 6 Days." Gamasutra. Retrieved July 19, 2011

(http://www.gamasutra.com/view/news/35957/Steam_Revenue_For_Zeboyd_Ga mes_RPGs_Outpaces_Lifetime_Xbox_Mark_In_6_Days.php).

Rose, Nikolas. 1999. The Powers of Freedom: Reframing political thought. Cambridge, UK: Cambridge University Press.

Rossi, Luca. 2009. "Playing your network: Gaming in social network sites." in Breaking New Ground: Innovation in Games, Play, Practice and Theory.

Rusli, Evelyn M. 2011a. "As Net Stocks Sizzle, Zynga Aims High for Its I.P.O.” The New York Times, online Retrieved January 10, 2012 (http://dealbook.nytimes.com/2011/07/01/zynga-files-for-1-billion-i-p-o/).

Rusli, Evelyn M. 2011b. “Zynga Nears Deal Valuing It at Close to \$10 Billion.” New York Times. Retrieved February 18, 2011

(http://dealbook.nytimes.com/2011/02/17/zynga-nears-deal-valuing-it-at-close-to10-billion/).

Ryan, Marie-Laure. 2004. Narrative Across Media: The languages of storytelling. Lincoln NE: University of Nebraska Press.

Salen, Katie, and Eric Zimmerman. 2004. Rules of Play: Game design fundamentals. Cambridge MA: The MIT Press.

Schell, Jesse. 2010a. "Design Outside the Box: Beyond Facebook." in DICE 2010. Retrieved (http://www.g4tv.com/videos/44277/dice-2010-design-outside-the-box- 
presentation/).

Schell, Jesse. 2010b. "TED TV: When games invade real life." Retrieved November 16, 2010

(http://www.ted.com/talks/jesse_schell_when_games_invade_real_life.html).

Schell, Jesse. 2008. The Art of Game Design: A book of lenses. Burlington MA: Morgan Kaufmann.

Schell, Jesse. 2010c. "Visions of the Gamepocalypse." FORA TV. Retrieved November 16,2010 (http://fora.tv/2010/07/27/Jesse_Schell_Visions_of_the_Gamepocalypse).

Schilling, Melissa A. 2003. "Technological Leapfrogging: Lessons from the U.S. video game industry.” California Management Review 45(3):6-32.

Schwab, Stephanie. 2010. "Gamification: What is it and why do you need to know?" Stephanie Schwab: Socialologist. Retrieved September 22, 2010 (http://www.stephanieschwab.com/2010/07/07/gamification/).

Shankar, Venkatesh, and Barry L. Bayus. 2003. "Network effects and competition: An empirical analysis of the home video game industry." Strategic Management Journal 24(4):375-384.

Sheff, David. 1993. Game Over: How Nintendo zapped an American industry, captured your dollars, and enslaved your children. New York City, N.Y.: Random House.

Sheffield, Brandon. 2010. "Super Rewards’ Bailey: ‘Quit Your Job And Make Facebook Games'." Gamasutra. Retrieved May 11, 2010 (http://www.gamasutra.com/view/news/28456/GDC_Canada_Super_Rewards_Ba iley_Quit_Your_Job_And_Make_Facebook_Games.php).

Shipman, Matt. 2011. "What Gamers Want: Researchers develop tool to predict player behavior." North Carolina State University Newsroom. Retrieved June 16, 2011 (http://news.ncsu.edu/releases/wmsrobertspredict).

Sicart, Miguel. 2010. "Against Procedurality.” in Homo Ludens: New space of socialization conference. Montréal, Canada.

Sicart, Miguel. 2011. "Against Procedurality.” Game Studies 11(3). Retrieved (http://gamestudies.org/1103/articles/sicart_ap).

Siegel, Scott Jon. 2010. "David v.s. Goliathville: Sage advice for indie social game designers." in Montréal International Games Summit. Montréal, Canada.

Siegel, Scott Jon. 2009. “Long-Tail Game Design: Building successful games for social 
networks." in Montréal International Games Summit. Montréal, Canada.

Silverman, Mark, and Bart Simon. 2009. "Discipline and Dragon Kill Points in the Online Power Game." Games and Culture 4(4):353-378.

Simon, Bart, Kelly Boudreau, and Mark Silverman. 2009. "Two Players: Biography and 'played sociality' in EverQuest." Game Studies 9(1). Retrieved (http://gamestudies.org/0901/articles/simon_boudreau_silverman).

Sinclair, Brenden. 2010. "Spot On: Social anxiety." Gamespot. Retrieved November 25, 2010 (http://www.gamespot.com/news/6284524.html).

Sliwinski, Alexander. 2012. “'38 Spouse' Explains Decision to Speak Now, Never Heard of 'EA Spouse'." Joystiq. Retrieved August 29, 2012 (http://www.joystiq.com/2012/06/14/38-studios-spouse-38-spouse/).

Sotamaa, Ollie. 2007. "On Modder Labour, Commodification of Play, and Mod Competitions." First monday 12(9). Retrieved (http://firstmonday.org/issues/issue12_9/sotamaa/index.html).

Sotamaa, Ollie. 2009. "The Player's Game: Towards understanding player production among computer game cultures." PhD Thesis, Tampere, Finland: University of Tampere.

Squire, Kurt. 2003. "Video Games in Education." International Journal of Intelligent Games \& Simulation 2(1).

Squire, Kurt, and Henry Jenkins. 2004. "Harnessing the Power of Games in Education." Insight 3(1):5-33.

Steinkuehler, Constance. 2006. "The Mangle of Play." Games and Culture 1(3):199 213.

Stone, A.R. 1995. The war of desire and technology at the close of the mechanical age. Cambridge, Mass.: MIT Press.

Strauss, Ben. 2011. "Violent Games Show Link in Altering Male Brains." Industry Gamers. Retrieved November 29, 2011 (http://www.industrygamers.com/news/violent-games-show-link-in-alteringmens-brains/).

Stuart, Keith. 2011. "The Metrics are the Message: How analytics is shaping social games." Guardian UK. Retrieved July 19, 2011 (http://www.guardian.co.uk/technology/gamesblog/2011/jul/14/social-gamingmetrics). 
Suits, Bernard. 1978. The Grasshopper: Games, Life and Utopia. Toronto, Canada: University of Toronto Press.

Suits, Bernard. 1967. "What is a Game?” Philosophy of Science 34(2):148 - 156.

Su, Mike. 2010. "Social Gaming: Where we've been, and where we're headed." TechCrunch. Retrieved November 15, 2010 (http://techcrunch.com/2010/11/13/social-gaming).

Surowiecki, James. 2004. Wisdom of Crowds. New York City, N.Y.: Random House.

Su, Susan. 2010. “Who's Using Facebook's Top Apps? Demographic data indicate diverse audiences." Inside Facebook. Retrieved June 21, 2010 (http://www.insidefacebook.com/2010/06/01/whos-using-facebooks-top-appsdemographic-data-indicate-diverse-audiences/).

Sutton-Smith, Brian. 1997. The Ambiguity of Play. Cambridge MA: Harvard University Press.

Takahashi, Dean. 2006. The Xbox 360 Uncloaked: The real story behind microsoft's nextgeneration video game console. Raleigh, N.C.: SpiderWorks LLC.

Takahashi, Dean. 2010. "Ubisoft to Launch Social Game Portal and First Facebook Game.” Venture Beat. Retrieved July 29, 2010 (http://venturebeat.com/2009/07/17/ubisoft-to-launch-social-game-portal-andfirst-facebook-game/).

Taylor, T.L. 2006a. "Does WoW Change Everything? How a PvP server, multinational player base, and surveillance mod scene caused me pause." Games and Culture $1(4): 318-337$.

Taylor, T.L. 2006b. Play Between Worlds: Exploring online game culture. Cambridge MA: The MIT Press.

Taylor, T.L. 2003. "Power Gamers Just Want to Have Fun?: Instrumental play in a MMOG." Pp. 300-311 in Level Up Conference Proceedings, edited by F Mayra, M. Copier, and J. Raessens. Utrecht, the Netherlands: University of Utrecht.

Taylor, T.L. 2007. "Pushing the Borders: Player participation and game culture." Pp. 112 - 130 in Structures of Participation in Digital Culture, edited by Joe Karaganis. New York City, N.Y.: Social Science Research Council.

Terdiman, Dan. 2010. "Why Zynga Ticks off the Games Industry." CNET News.

Retrieved July 22, 2010 (http://news.cnet.com/8301-13772_3-20002221-52.html).

Terranova, Tiziana. 2000. "Free Labor: Producing culture for the digital economy." 
Social Text 18(2):33-58.

Thomsen, Michael. 2011. "We're Going to Need Another Category: Going hardcore on Facebook." Gamasutra. Retrieved October 12, 2011

(http://www.gamasutra.com/view/feature/6507/were_going_to_need_another_.ph p?print=1).

Totilo, Stephen. 2008. "Low Metacritic Scores Cause Game Publishers To Withhold Developer Royalties." MTV Geek. Retrieved January 26, 2012 (http://multiplayerblog.mtv.com/2008/05/29/low-metacritic-costs-developers/).

Tschang, F. Ted. 2005. "Videogames as Interactive Experiential Products and their Manner of Development." International Journal of Innovation Management $9(1): 103-131$.

Tychsen, Anders. 2008. "Crafting User Experience via Game Metrics Analysis.” in Proceedings of NordiCHI: Research goals and strategies for studying user experience and emotion workshop. Lund, Sweden Retrieved (http://www.cs.uta._/ ux-emotion).

Valadares, Jeferson. 2011. "Consumers Spend Average of \$14 per Transaction in iOS and Android Freemium Games.” Flurry.com. Retrieved July 27, 2011 (http://blog.flurry.com/bid/67748/Consumers-Spend-Average-of-14-perTransaction-in-iOS-and-Android-Freemium-Games).

Valverde, Mariana. 2008. "Beyond Discipline and Punish: Foucault's challenge to criminology." Pp. 201-223 in Discipline, Security, and Beyond: Rethinking Michel Foucault's 1978 \& 1979 College de France Lectures, vol. 4, Carceral Notebooks, edited by Bernard E. Harcourt. Chicago, Ill.: Bernard E. Harcourt Retrieved (http://www.thecarceral.org/journal-vol4.html).

Vanden Bossche, Andrew. 2011. "Trapdoor Studios On Warp And Small-Scale Development." Gamasutra. Retrieved May 4, 2011 (http://www.gamasutra.com/view/news/33970/Interview_Trapdoor_Studios_On_ Warp_And_SmallScale_Development.php).

Weber, Max. 2002. The Protestant Ethic and the Spirit of Capitalism: and Other Writings. New York City, N.Y.: Penguin Classics.

Webster, Andrew. 2011a. "Smartphone and Tablet Rundown: What developers need To know." Gamasutra. Retrieved July 27, 2011 (http://www.gamasutra.com/view/feature/6440/smartphone_and_tablet_rundown_ .php?print=1).

Webster, Andrew. 2011b. "The Crowdfunding Revolution: Perspectives." Gamasutra. Retrieved April 22, 2011 
(http://www.gamasutra.com/view/feature/6353/the_crowdfunding_revolution_.ph p).

Webster, Andrew. 2011c. "Xbox Live Indie Games: No way to make a living." Ars Technica. Retrieved July 6, 2011 (http://arstechnica.com/gaming/news/2011/07/xblig-feature.ars).

Wenger, Etienne, Richard Arnold McDermott, and William Snyder. 2002. Cultivating Communities of Practice: A Guide to Managing Knowledge. Boston, Mass: Harvard Business Press.

Whitson, Jennifer R. 2011. "La Révolution Des Jeux Sociaux.” in Socialisation et Communication dans les Jeux Vidéo, edited by C. Perraton, M. Fusaro, and M. Bonenfant. Montréal: Les Presses de l’Université de Montréal.

Whitson, Jennifer R. 2010a. "Rule Making and Rule Breaking: Game Development and the Governance of Emergent Behaviour." fibreculture (16). Retrieved (http://sixteen.fibreculturejournal.org/rule-making-and-rule-breaking-gamedevelopment-and-the-governance-of-emergent-behaviour/).

Whitson, Jennifer R. 2010b. "Surveillance and Democracy in the Digital Enclosure.” Pp. 231-246 in Surveillance and Democracy, edited by Kevin D. Haggerty and Minas Samatas. Oxford: Routledge.

Whitson, Jennifer R., and Claire Dormann. 2011. "Social Gaming for Change: Facebook unleashed." First Monday 16(10). Retrieved October 4, 2011 (http://firstmonday.org/htbin/cgiwrap/bin/ojs/index.php/fm/article/view/3578/305 8).

Whitson, Jennifer R., and Aaron Doyle. 2008. "Second Life and Governing Deviance in Virtual Worlds." Pp. 88-111 in Technocrime: Technology, Crime, and Social Control, edited by S. Leman-Langlois. Cullompton, Devon: Willan Publishing.

Wikipedia. 2010a. "Gamification.” Wikipedia. Retrieved September 22, 2010 (http://en.wikipedia.org/wiki/Gamification).

Wikipedia. 2010b. "Gamification.” Retrieved November 16, 2010 (http://en.wikipedia.org/wiki/Gamification).

Wikipedia. 2012. “Gamification.” Wikipedia. Retrieved March 9, 2012 (http://en.wikipedia.org/wiki/Gamification).

Wikipedia. 2011. "World of Warcraft." Wikipedia. Retrieved February 8, 2011 (http://en.wikipedia.org/wiki/World_of_Warcraft).

Williams, Dmitri. 2002. "Structure and Competition in the U.S. Home Video Game 
Industry." The International Journal on Media Management 4(1):41-54.

Williams, M.H. 2011. “Gameloft Accused Of 'Dangerous' Work Conditions.” Industry Gamers. Retrieved July 19, 2011

(http://www.industrygamers.com/news/gameloft-accused-of-dangerous-workconditions/).

Wilson, Douglas, and Miguel Sicart. 2009. "Abusing the Player, and Making Them Like it Too!" in DiGRA '09: Breaking New Ground: Innovation in Games, Play, Practice and Theory. Retrieved (http://www.youtube.com/watch? $\mathrm{v}=$ YB9EoNa9bkA).

Wilson, Douglas, and Miguel Sicart. 2010. "Now It's Personal: On Abusive Game Design." in Proceedings of Future Play '10. New York City, N.Y.: ACM.

Windels, Joel. 2011. "Scary Game Findings: A study of horror games and their players." Gamasutra. Retrieved September 7, 2011

(http://www.gamasutra.com/view/feature/6480/scary_game_findings_a_study_of_ .php?print=1).

Winner, Langdon. 1986. "Do Artifacts Have Politics?” Pp. 19-39 in The Whale and the Reactor: A search for limits in an age of high technology. Chicago IL: University of Chicago Press.

Wolf, Gary. 2010. "The Data-Driven Life." New York Times. Retrieved September 14, 2010 (http://www.nytimes.com/2010/05/02/magazine/02self-measurement-t.html? ${ }^{\mathrm{r}}=1$ \&pagewanted=all).

Yee, Nick. 2006a. "Motivations of Play in Online Games." CyberPsychology and Behavior 9(6):772-775.

Yee, Nick. 2006b. "The Labor of Fun: How video games blur the boundaries of work and play." Games and Culture 1(1):68 - 71 .

Young, Neil. 2010. "Things to Unlearn Moving from Traditional Development to the New Digital World." in GDC 2010, GDC Vault. Retrieved May 11, 2010 (http://www.gdcvault.com/free/category/280/conference/).

Zichermann, Gabe, and Christopher Cunningham. 2011. Gamification by Design: Implementing game mechanics in web and mobile apps. Sebastopol, CA: O'Reilly Media, Inc.

Zichermann, Gabe, and Joselin Linder. 2010. Game-Based Marketing: Inspire customer loyalty through rewards, challenges, and contests. Hoboken N.J.: Wiley.

Zoeller, Georg. 2010. “GDC 2010: Development telemetry in video games projects.” 
Georg@GDC. Retrieved March 22, 2011 (http://gdc.gulbsoft.org/talk). 